\title{
RF FRONT END FOR AN INTEGRATED SILHOUETTE CAPTURE AND BOUNDARY DETECTION FREQUENCY MODULATED CONTINUOUS WAVE ULTRA-WIDEBAND RADAR SYSTEM FOR THE EXTENSION OF INDEPENDENT LIVING
}

\author{
A Thesis \\ presented to \\ the Faculty of California Polytechnic State University, \\ San Luis Obispo
}

\author{
In Partial Fulfillment \\ of the Requirements for the Degree \\ Master of Science in Electrical Engineering
}

by

Adrian Smet

December 2017 
(C) 2017

Adrian Smet

ALL RIGHTS RESERVED 


\section{COMMITTEE MEMBERSHIP}

TITLE:

AUTHOR:

DATE SUBMITTED:

COMMITTEE CHAIR:

COMMITTEE MEMBER:

COMMITTEE MEMBER:
RF Front End for an Integrated Silhouette Capture and Boundary Detection Frequency Modulated Continuous Wave Ultra-Wideband Radar System for the Extension of Independent Living

Adrian Smet

December 2017

Tina Smilkstein, Ph.D.

Associate Professor of Electrical Engineering

Dennis Derickson, Ph.D.

Department Chair of Electrical Engineering

William Ahlgren, Ph.D.

Associate Professor of Electrical Engineering 


\begin{abstract}
RF Front End for an Integrated Silhouette Capture and Boundary Detection Frequency Modulated Continuous Wave Ultra-Wideband Radar System for the Extension of Independent Living Adrian Smet
\end{abstract}

Limitations of current eldercare monitoring systems leave a need for new solutions. A monitoring system based on a frequency modulated continuous wave ultra-wideband short-range radar is proposed for this application. The complete proposed monitoring system is comprised of four blocks: boundary detection, silhouette capture, human identification, and data transmission. This paper develops the RF front end hardware for the silhouette capture subsystem.

System requirements are derived for the silhouette capture subsystem. An architecture for the RF front end is designed, and required individual component specifications are determined. Components are selected off the shelf or custom designed for each socket. Full transmitter and receiver level plans are calculated to ensure expected system performance meets system requirements. A component library and full system schematic is created, PCB layout is completed, and PCB files are generated and sent for fabrication. PCB traces and individual components are characterized over frequency, and methods that improve inadequate performance are documented and discussed. 


\section{TABLE OF CONTENTS}

LIST OF TABLES

Page

LIST OF FIGURES viii

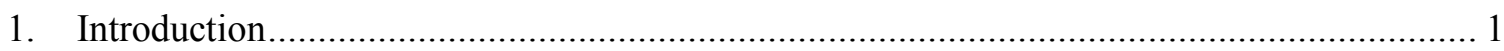

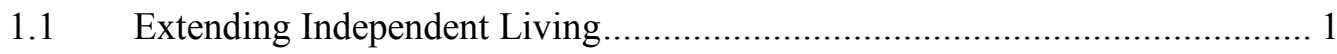

1.2 Current Eldercare Monitoring Methods.......................................................... 1

1.3 Required Improvements for Monitoring Systems ......................................... 2

1.4 Application of Radar to Extending Independent Living................................ 2

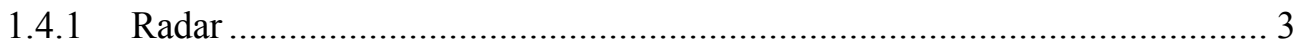

1.4.2 Radar Advantages in Monitoring ................................................................ 3

1.4.3 Custom Eldercare Monitoring Radar Application .................................. 3

1.4.4 Proposed Monitoring System Functions.................................................. 4

1.4.5 Proposed System Functional Blocks ......................................................... 4

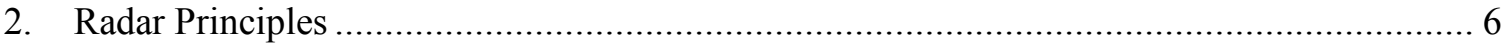

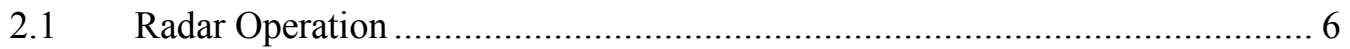

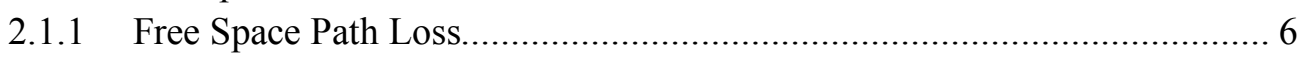

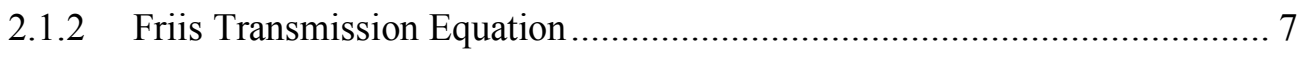

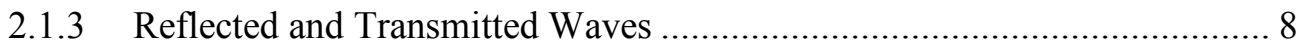

2.2 Required System Outputs for Silhouette Capture ........................................ 9

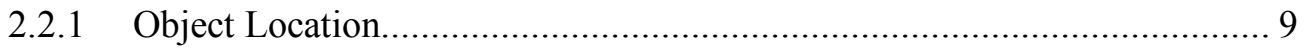

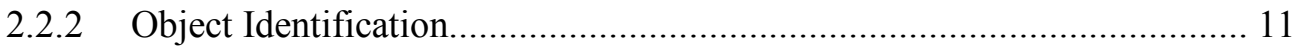

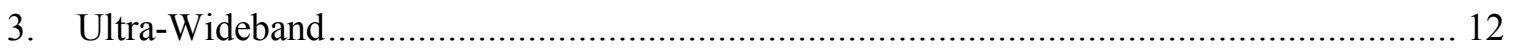

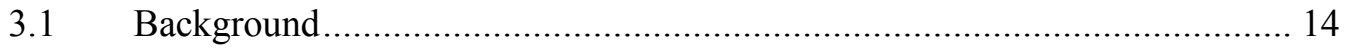

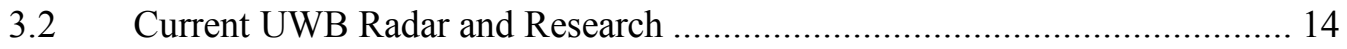

3.3 Human Identification with UWB ........................................................ 16

3.3.1 Human Body Tissue Properties ...................................................... 17

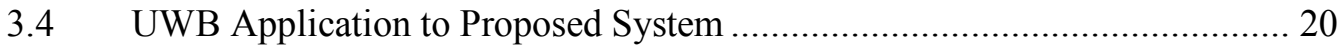

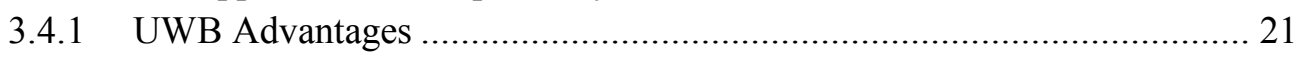

3.4.2 System Frequency Spectrum ....................................................... 21

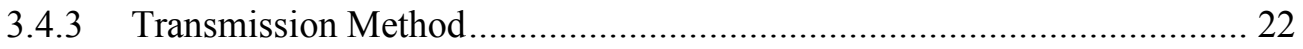

3.5 Frequency Modulated Continuous Wave ................................................. 23

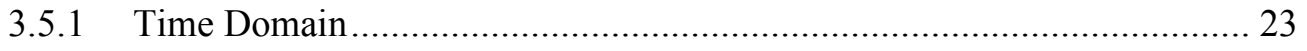

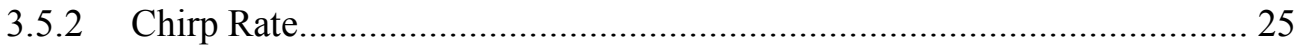

3.5.3 Time Delay and Frequency Offset ................................................... 25

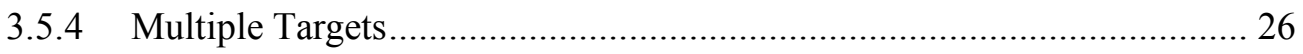

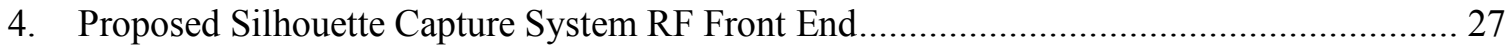

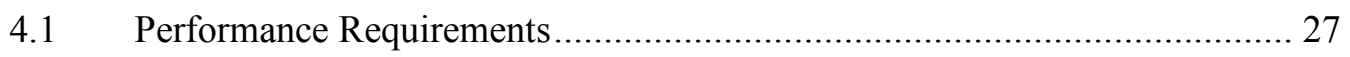

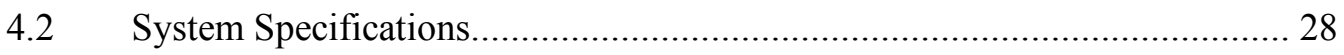

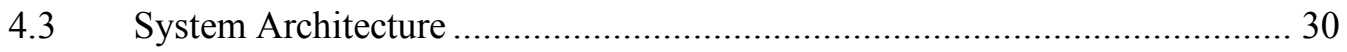

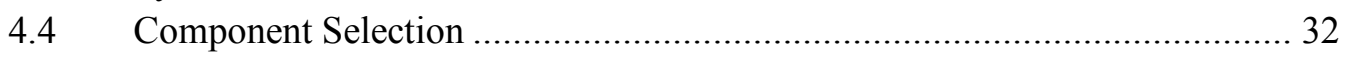

4.4.1 Component Parameter Discussion .................................................... 32 


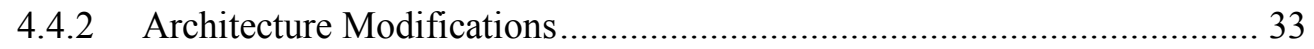

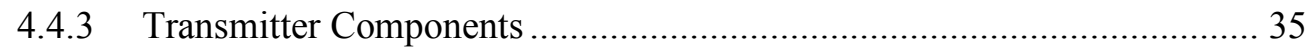

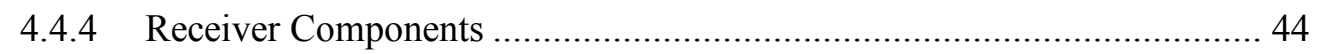

4.4.5 Component DC Voltage Supplies ....................................................... 53

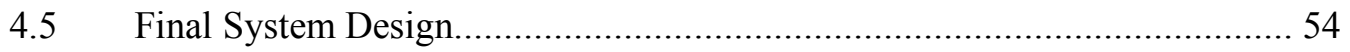

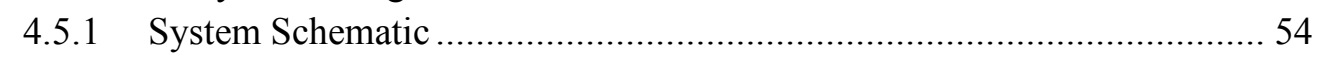

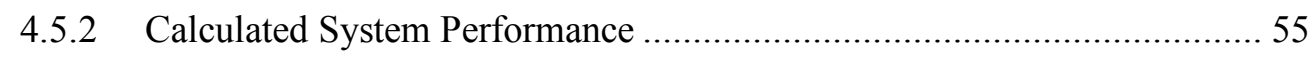

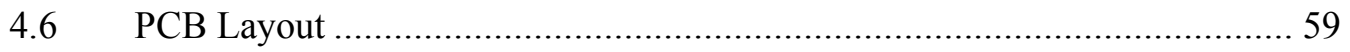

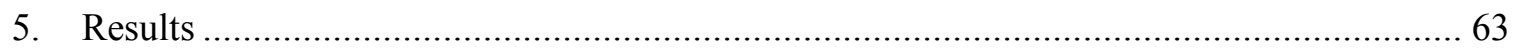

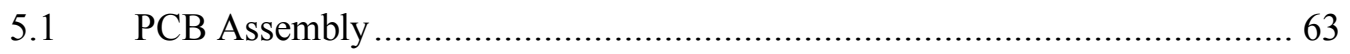

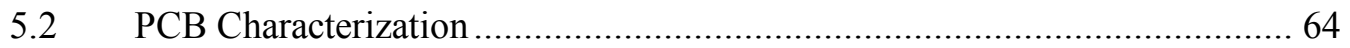

5.3 Transmitter Component Characterization.....................................................6 68

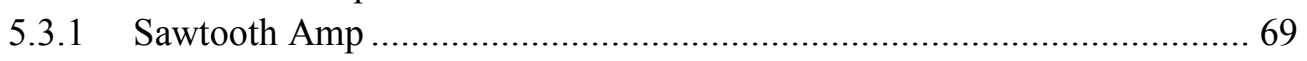

5.3.2 Voltage Controlled Oscillator............................................................ 70

5.3.3 Low Frequency Tx Amplifier and Input Attenuator ................................ 72

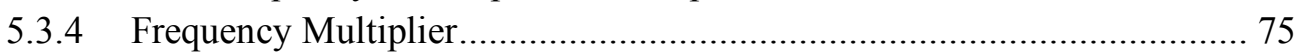

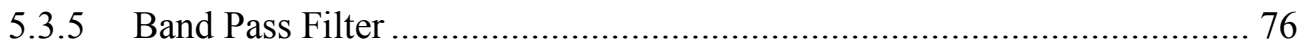

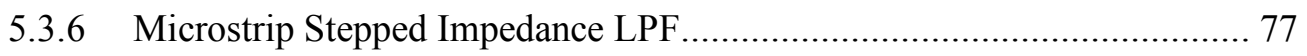

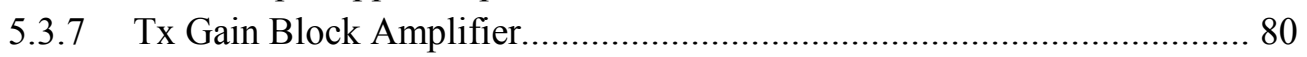

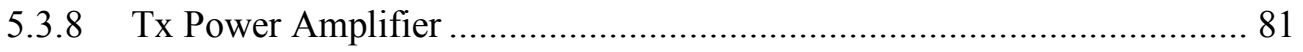

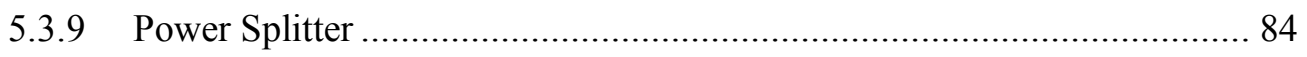

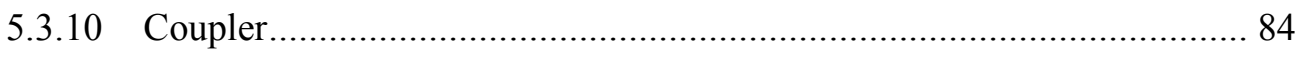

5.3 .11 Output Power Attenuator .............................................................. 86

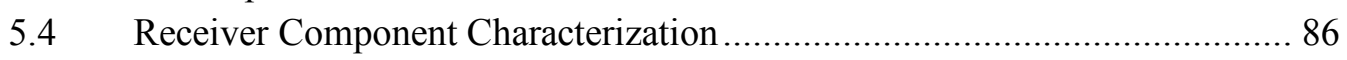

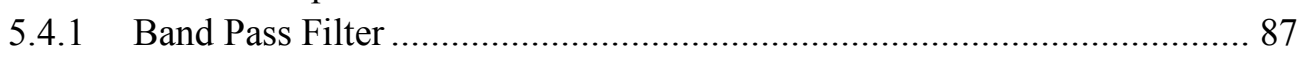

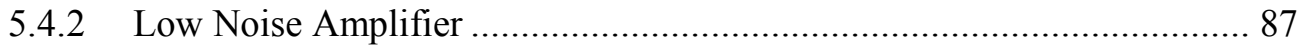

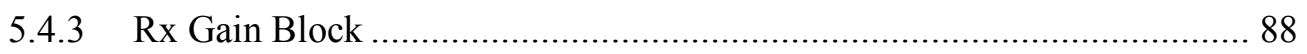

5.4.4 Mixer .......................................................................................... 90

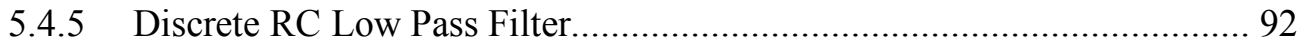

5.4.6 Active Low Pass Filter .......................................................................... 93

5.4.7 Variable Gain Amplifier...................................................................... 95

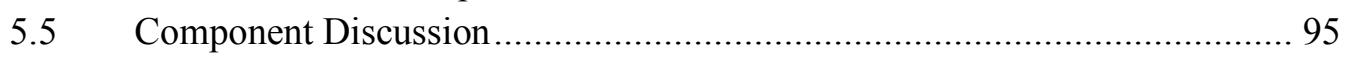

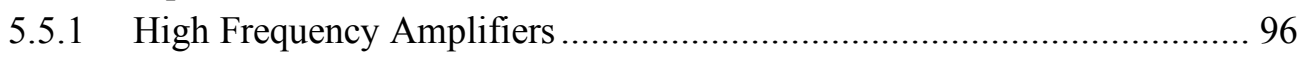

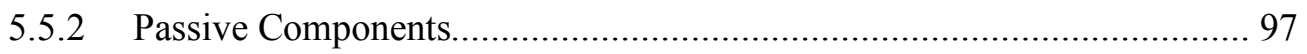

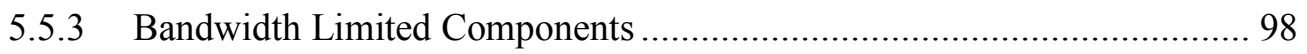

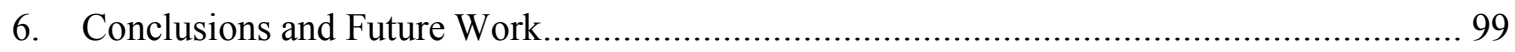

6.1 Silhouette Capture RF Front-End Future Improvements............................100

6.2 Remaining Silhouette Capture Subsystem Projects ..................................102

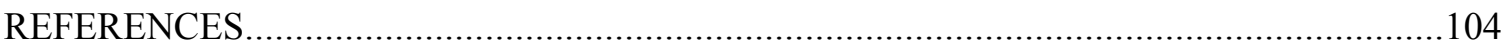

APPENDICES

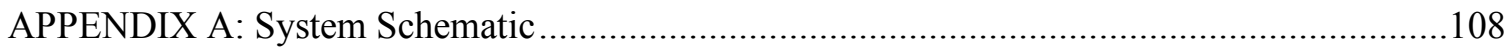

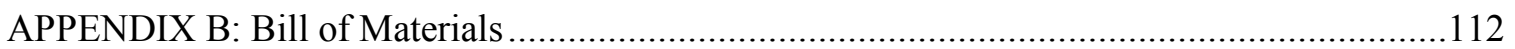




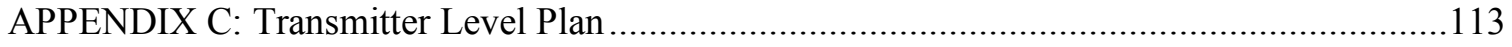

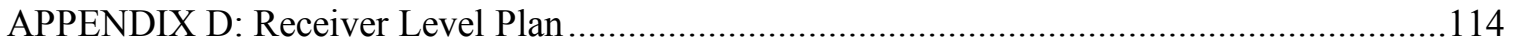

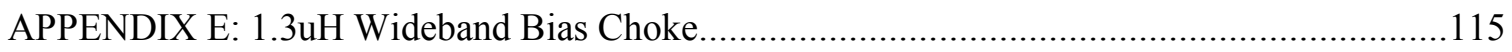

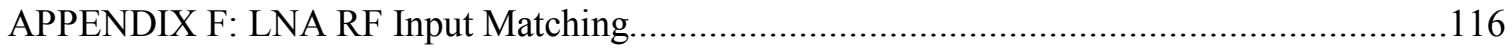

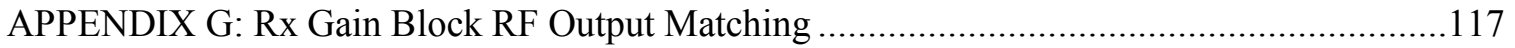

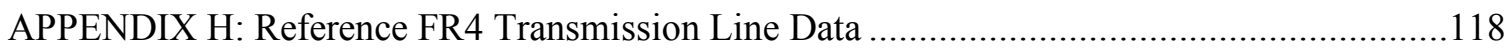

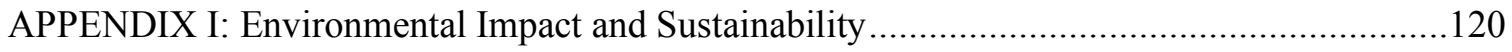




\section{LIST OF TABLES}

$\begin{aligned} & \\ & \text { Page }\end{aligned}$

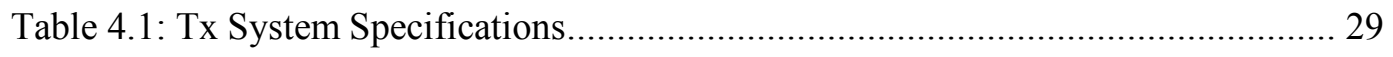

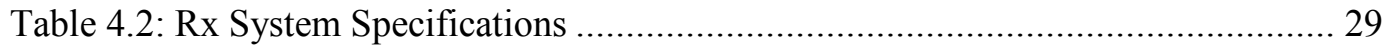

Table 4.3: SKY67014-396LF Datasheet Specifications, Icq=18mA [41] ..................... 38

Table 4.4: TDK UWB BPF DEA453960BT-3002B1 Datasheet Specifications [43]..... 40

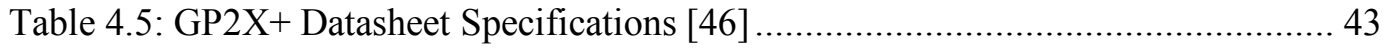

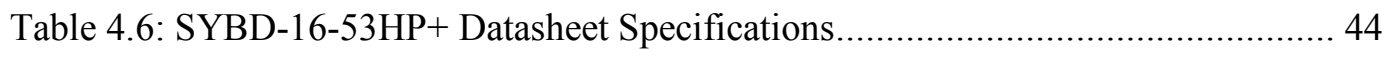

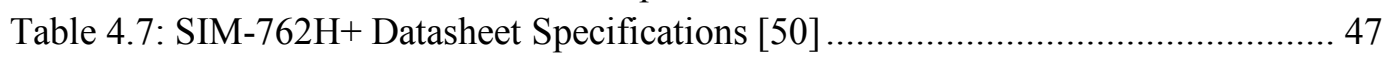

Table 4.8: Active LPF Schematic Values...................................................................... 51

Table 4.9: LMH6639 Typical Datasheet Performance [52] ........................................ 51

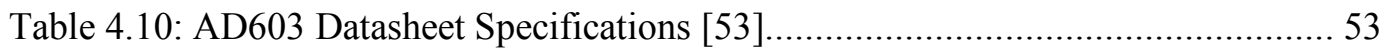




\section{LIST OF FIGURES}

Page

Figure 2.1: Triangulation for Object Location..................................................... 10

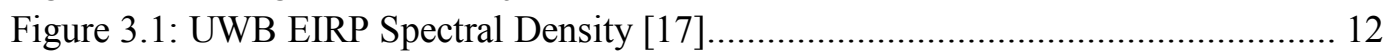

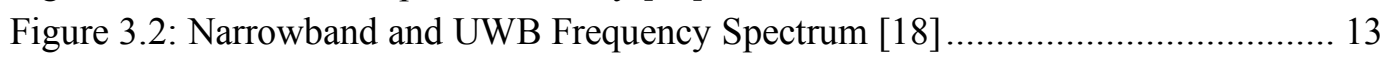

Figure 3.3: Time Domain Representations of Narrowband and UWB Signals [18]....... 13

Figure 3.4: Radar Return Spectral Variations from a Metal Sheet [6] .......................... 15

Figure 3.5: Radar Return Spectral Variations from a Breathing Person [6] ................... 15

Figure 3.6: Radar Return Spectral Variations from a Non-Breathing Person [6]............ 16

Figure 3.7: Initial Human Body Tissue Layers....................................................... 17

Figure 3.8: Reflected and Transmitted Waves through a Human Body [24] .................. 18

Figure 3.9: Relative Permittivity of Human Body Tissues [25] [26] [27] [28] .............. 19

Figure 3.10: Received Reflected Power from a Human Body over Frequency [29] ....... 20

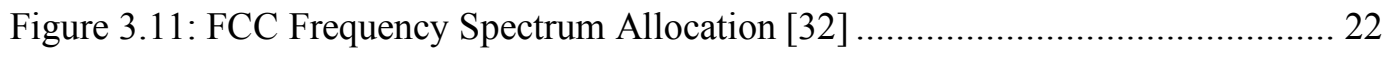

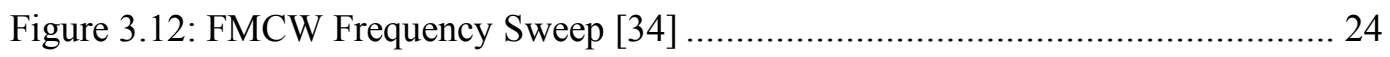

Figure 3.13: Time Domain FMCW Transmit Signal ................................................. 24

Figure 3.14: Transmit and Received FMCW Signals [36] ...................................... 25

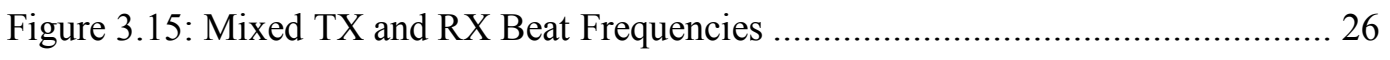

Figure 4.1: Transmitter System Architecture ............................................................... 30

Figure 4.2: Receiver System Architecture ........................................................... 31

Figure 4.3: Final Transmitter System Block Diagram .............................................. 34

Figure 4.4: Final Receiver System Block Diagram ................................................... 35

Figure 4.5: Non-Inverting Op Amp [37] ........................................................... 35

Figure 4.6: ROS-2700-1819+ Datasheet Frequency Tuning Voltage [40]..................... 36

Figure 4.7: ROS-2700-1819+ Datasheet Pout and Harmonic Levels [40] ...................... 37

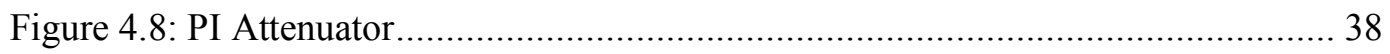

Figure 4.9: KSX2-722+ Typical Datasheet Performance [42] …................................. 39

Figure 4.10: Stepped Impedance LPF ADS Simulation........................................... 40

Figure 4.11: Stepped Impedance LPF Simulation Results ...................................... 41

Figure 4.12: ADL5541 Typical Datasheet Performance [44] ...................................... 41

Figure 4.13: MGA-83563 Typical Datasheet Performance vs. Frequency [45] .............. 42

Figure 4.14: MGA-83563 Typical Datasheet Performance vs. Input Power [45] .......... 42

Figure 4.15: GP2X+ Typical Datasheet Total Loss and Isolation [46] .......................... 43

Figure 4.16: SYBD-16-53HP+ Typical Datasheet Loss Coupling and Directivity [47].. 44

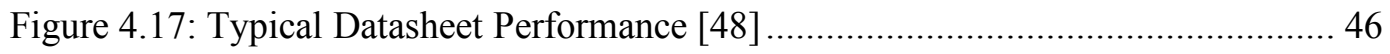

Figure 4.18: AD5542 Typical Datasheet Performance [49] ..................................... 47

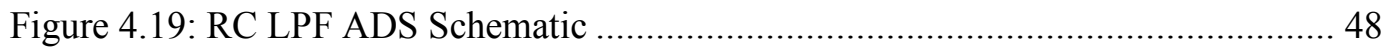

Figure 4.20: RC LPF ADS Simulation Results ...................................................... 49

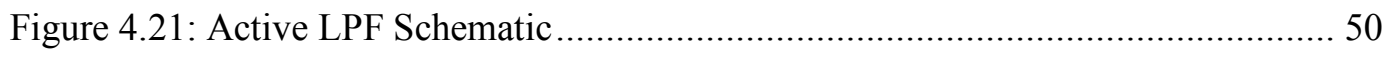

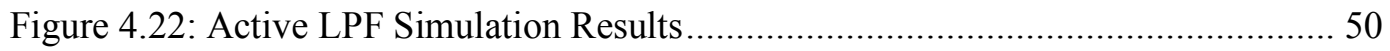

Figure 4.23: Micrel MIC5335 Datasheet Specifications [54] ..................................... 54

Figure 4.24: Higher Order Mixing Products [55] .................................................. 58 


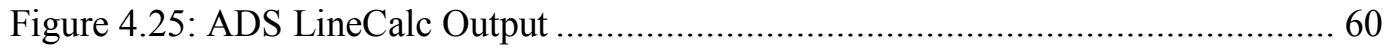

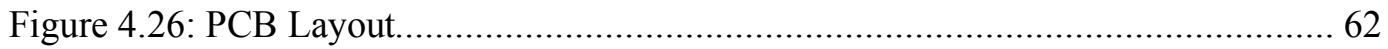

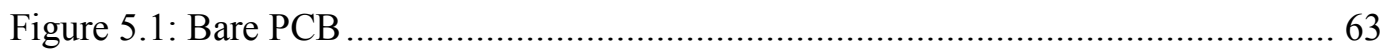

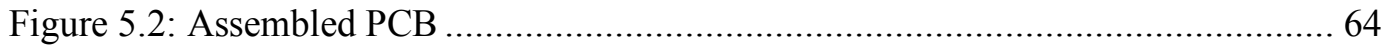

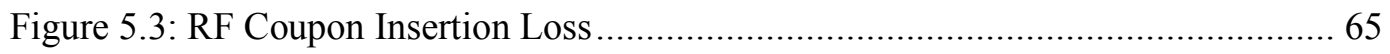

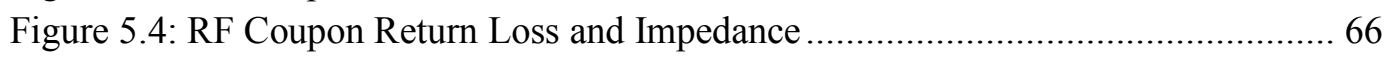

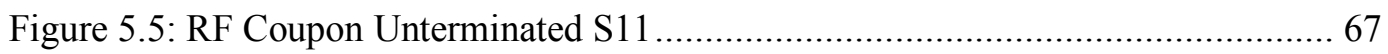

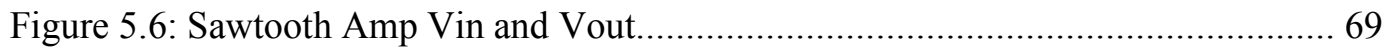

Figure 5.7: Sawtooth Amp Gain ............................................................................. 70

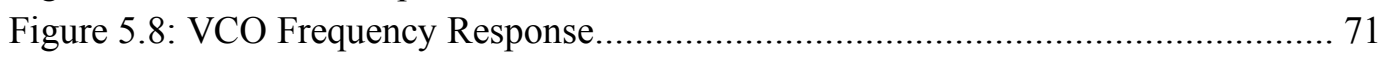

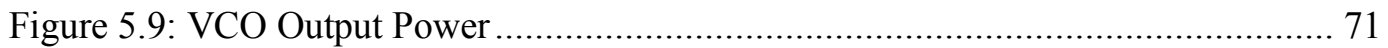

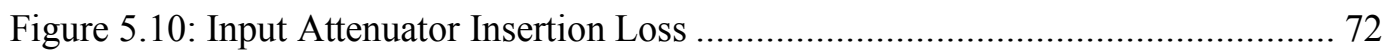

Figure 5.11: Input Attenuator Return Loss................................................................ 73

Figure 5.12: Low Frequency Tx Amplifier Gain ..................................................... 73

Figure 5.13: Low Frequency Tx Amplifier Return Loss.............................................. 74

Figure 5.14: Low Frequency Tx Amplifier Input and Output Impedance ...................... 74

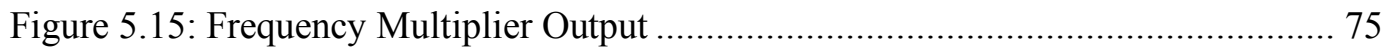

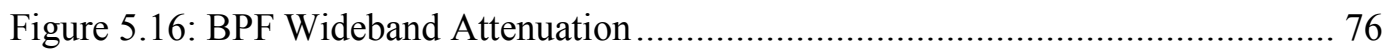

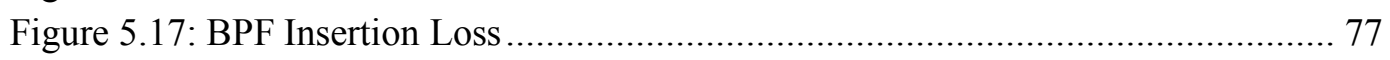

Figure 5.18: Microstrip Stepped Impedance LPF Measured Frequency Response ......... 78

Figure 5.19: Microstrip Filter Momentum Simulation............................................... 79

Figure 5.20: Microstrip Filter Momentum Simulation Frequency Response .................. 79

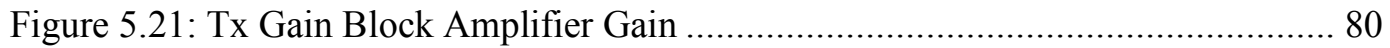

Figure 5.22: Tx Gain Block Amplifier Return Loss and Impedance ............................ 81

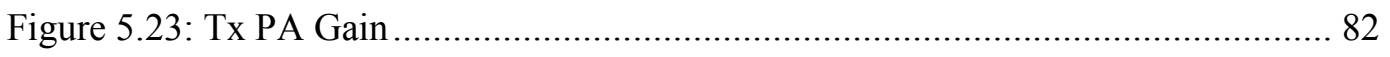

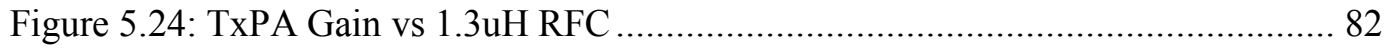

Figure 5.25: TxPA Gain vs Measurement Location .................................................. 83

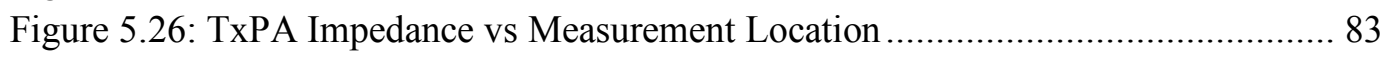

Figure 5.27: TxPA Return Loss vs Measurement Location ........................................ 84

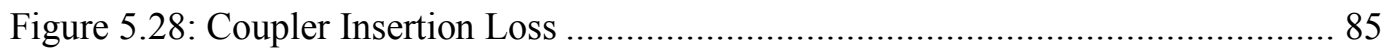

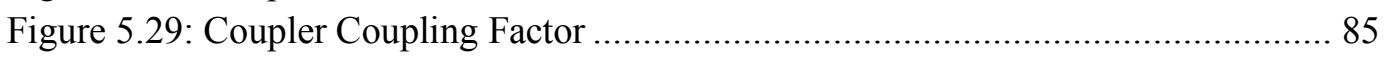

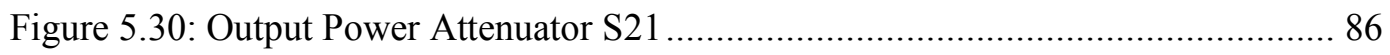

Figure 5.31: LNA Gain with 1.3uH RFC and Measurement Location .......................... 87

Figure 5.32: LNA Impedance with 1.3uH RFC and Measurement Location................... 88

Figure 5.33: LNA Return Loss with 1.3uH RFC and Measurement Location ................ 88

Figure 5.34: Rx Gain Block Amplifier Gain vs 1.3uH RFC ....................................... 89

Figure 5.35: Rx Gain Block Amplifier Return Loss and Impedance vs 1.3uH RFC ....... 89

Figure 5.36: Mixer Down Converted Power vs IF Frequency, RF=3GHz.................... 90

Figure 5.37: Mixer Down Converted Power vs RF Frequency, $\mathrm{IF}=3 \mathrm{MHz}$................... 90

Figure 5.38: Mixer IF Port Leakage and Harmonics ................................................. 91

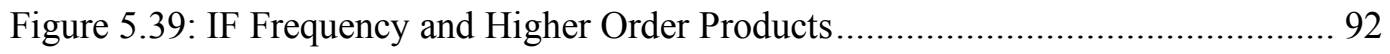

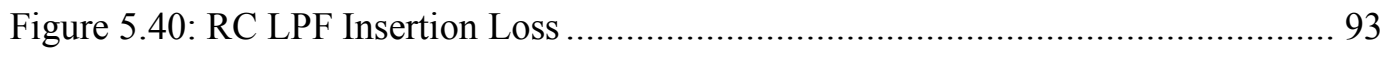




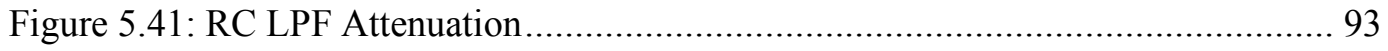

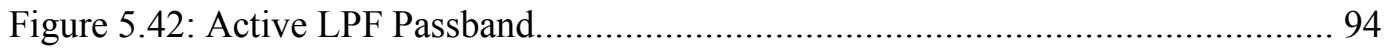

Figure 5.43: Active LPF Attenuation.................................................................... 94

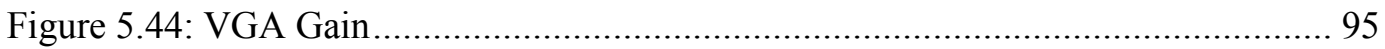

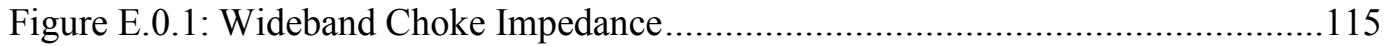

Figure H.0.1: Measured Reference FR4 Microstrip Transmission Line .........................118

Figure H.0.2: Measured Reference FR4 Transmission Line Insertion Loss...................118

Figure H.0.3: Measured Reference FR4 Transmission Line RL and Impedance ...........119 


\section{Introduction}

\subsection{Extending Independent Living}

The idea of extending independent living, allowing the ill or elderly to live within their own means, offers many benefits to society. Requiring individuals to live in a care facility or having a caregiver come live with them has multiple downsides. The quickly aging population, combined with an insufficient number of caretakers leads to a high cost of assisted living. From the financial perspective, less dedicated support for each individual lower cost. Additionally, individuals like living independently and would prefer minimal impact on their lives when needing care. The solution to these problems is using more automated systems to help extend independent living. With advancements in technology, this application can be further developed to provide high quality of life to all individuals for longer periods of time.

\subsection{Current Eldercare Monitoring Methods}

There are multiple monitoring systems which currently attempt to extend independent living by providing monitoring of an individual and alerts when something is wrong. One of the most important functions of a monitoring system is the ability to provide instant real-time alerts to allow quick response times which prevents caretaking from needing to be present at all times. An equally important function is to gather detailed health data of the patients over time, which allows healthcare specialists to preemptively identify and treat health issues [1] [2].

Current systems that attempt to achieve these two goals can be divided into a few highlevel categories: cameras, wearables, and household sensors. Cameras can be used to monitor individuals for falls and other emergencies as well as track behavioral habits. Wearable sensors can include blood pressure and heart rate monitors, and other wearables allow the individual to 
call for help in emergencies. Sensors placed throughout the house can monitor safety featuresincluding heat sensors on ovens and stoves and water flow detection [3].

\subsection{Required Improvements for Monitoring Systems}

The monitoring systems described in the previous section have a number of drawbacks which can be improved upon. Systems that use visual surveillance with cameras are limited by line of sight. Additional cameras need to be placed around every corner to ensure a complete monitoring area. Cameras also require enough ambient light to get a clear picture, so their uses

are limited at night. Another limitation with cameras is the individual's privacy, video surveillance is an invasive method which many individuals are uncomfortable with [4].

Wearables, which can provide accurate health data for an individual, are also invasive. They require placement on the individuals body, and depending on the sensor can be an obstruction to comfortable living. Additionally, they require actions from the individual ranging from putting the device on every day, to cleaning, or installation of batteries.

Household sensors can provide data for their specific individual tasks, but are unable to provide a complete picture. Many sensors are required to be installed for various functions, and still additional monitoring methods are often needed to complement them [5].

\subsection{Application of Radar to Extending Independent Living}

Based off of the limitations of current systems and the required improvements for new monitoring systems, a new radar based monitoring system is proposed. This section discusses how radar works, why it is a good candidate for a monitoring system, and the functional system blocks that would make up this new radar based monitoring system. 


\subsubsection{Radar}

Radar systems use electromagnetic waves to detect and identify objects. They transmit a signal in the direction of interest, which propagates out through the air until it reaches an object. Upon reaching an object, part of the signal will be reflected back towards the radar system and part of the signal will continue through the object, depending on the object's material properties. The radar system receives these reflected signals and uses them to determine characteristics of the object such as size, shape, location, and material properties.

\subsubsection{Radar Advantages in Monitoring}

Radar bring many advantages to the application of eldercare monitoring. Because it uses electromagnetic waves for detection, it is inherently non-intrusive. No visual images are generated as part of its usage, so full privacy is maintained. Because electromagnetic waves are

able to propagate through walls, a single radar unit is able to monitor multiple rooms and does not depend on ambient lighting. Additionally, accurate high frequency radars have been demonstrated to be able to monitor health data such as breathing patterns and even heart rate [6]. Radar's ability to provide all desired facets of monitoring in an unobtrusive way with a single unit means it can greatly reduce the complexity and cost of eldercare monitoring while maintaining full privacy. Its flexibility and accuracy allows it to improve health data quality and behavior tracking to facilitate healthcare specialists in preemptively identifying potential issues.

\subsubsection{Custom Eldercare Monitoring Radar Application}

There is currently a lot of research being done regarding using radar for human being detection. This paper proposes a radar monitoring system with the features discussed below in Section 1.4.4. A custom design is pursued for this radar system for multiple reasons. It allows 
facilitation of all requested system features as well as allows for expansion into future features and projects for this system. A major example here is expanding this project for human detection, as most monitoring systems do not have the goal of identifying specific individuals apart from each other. Additionally, customization allows the system to be optimized for the specific application which means the hardware is not wasting resources such as power, cost, or complexity on unneeded features. Application specific system requirements such as range, desired target types, and power usage are all considered in the system design phase and used to derive specifications for each subcomponent in the system.

\subsubsection{Proposed Monitoring System Functions}

Proposed is a system architecture for a radar based monitoring system for eldercare monitoring with the purpose of extending independent living. The system is required to track the silhouettes of human targets inside a defined monitoring region. It must also be able to differentiate the human target from other humans in monitoring region. By tracking the target's silhouette, it will be able detect events such as falls and send alerts. It will also be able to log daily actions such as hygiene patterns and social interactions to determine cognitive decline, as well as sleep patterns and sleep restlessness. Furthermore, the proposed system will be able to measure general physical health by monitoring respiratory rate and potentially even heart rate.

\subsubsection{Proposed System Functional Blocks}

In order to achieve the functions outlined above, a system with four discrete functional blocks is proposed. The four functional blocks are boundary detection, silhouette capture, human identification, and data transmission.

The boundary detection functional block is used to initialize the system. It identifies a specific region to monitor for targets, such as a series of rooms. This allows the system to focus 
in certain areas, reducing processing complexity and false detections. It reduces multipath noise by ignoring radar signals that are reflected from outside of the defined monitoring region.

The second functional block, silhouette capture, is used to locate targets. The returned radar reflections off an object in the monitoring region is used to position it. The target's position is tracked over time to monitor behavior and identify events such as falls. With enough accuracy in the silhouette capture block, vital signs such as respiratory rate and heart rate can be monitored as well.

The purpose of the human identification functional block is to differentiate human targets from one another. At any given time, there may be multiple humans inside the system monitoring region. The human identification block is able to identify the specific target or targets which are meant to be monitored. This prevents invalid data from being logged from the wrong targets who may enter the monitoring region.

Finally, the data transmission functional block is responsible for sending processed radar data from the radar system unit to a central processing unit for analysis. A dedicated processing unit allows combining of information collected from multiple radar systems, and the application of complex processing intense algorithms to the data to meet the required system features.

The following chapters discuss a proposed operation for the silhouette capture subsystem. An RF Front End is architected, components are selected and designed, and a PCB is fabricated and tested. 


\section{Radar Principles}

In order to gather information about an object, radar systems use information from reflected electromagnetic waves such as propagation time and amount of power reflected. In order to trace information from the reflected waves back into characteristics of the object that caused the reflection, how the waves behave as they travel through space and encounter objects must be understood.

\subsection{Radar Operation}

In order to understand the requirements of a silhouette capture radar system, first we will consider how electromagnetic waves travel through space and how they react when encountering an object in their path.

\subsubsection{Free Space Path Loss}

Free space path loss (FSPL) is measured as the ratio of the transmitted power to the received power as the signal propagates though free space between two separated points. The received power depends on the available power density at that point in space, as well as the receiver's ability to capture that available power. As a signal propagates out from an ideal isotropic radiator, the power spreads equally over the surface area of a sphere, given by Eqn 2.1.

$$
A=4 \pi R^{2}
$$

Eqn 2.1 
Thus, the power density in a particular unit area decreases the further it is from the source, as it is inversely proportional to the square of the distance.

The ability of the receiver to capture the available power is proportional to the square of the signal wavelength divided by $4 \pi$ [7]. Therefore, the total free space path loss depends on distance to the target as well as signal wavelength, as given in Eqn 2.2.

$$
F S P L=\left(\frac{4 \pi R}{\lambda}\right)^{2}
$$

Eqn 2.2

\subsubsection{Friis Transmission Equation}

Building off of the free space path loss analysis, the received power of a signal that is transmitted and reflects off an object can be calculated. At the object edge the power density, $\omega_{\mathrm{r}}$, is given by Eqn 2.3, where $P_{t}$ is the transmit power, and $G_{t}$ is the directional gain of the transmit antenna in the direction of the object [8].

$$
\omega_{r}=\frac{P_{t} G_{t}}{4 \pi R^{2}}, \quad\left[\frac{W}{m^{2}}\right]
$$

Eqn 2.3

The power reflected off the object back towards the antenna, denoted $\mathrm{P}_{\mathrm{t} 2}$, is given by Eqn 2.4, where $\sigma$ is the radar cross section (RCS) of the object in the direction of the antenna in units of $\mathrm{m}^{2}$.

$$
P_{t 2}=\omega_{r} \sigma, \quad[W]
$$

Eqn 2.4

Similar to Eqn 2.3 the power density at the receiver, $\omega_{\mathrm{r} 2}$, is

$$
\omega_{r 2}=\frac{P_{t 2}}{4 \pi R^{2}}, \quad\left[\frac{W}{m^{2}}\right]
$$

Eqn 2.5 
The effective antenna aperture, $A_{e}$, determines the amount of available power that will actually be captured by the receive antenna. The effective aperture shown in Eqn 2.6 depends on the gain of the receive antenna, $\mathrm{G}_{\mathrm{r}}$, and the signal wavelength, $\lambda[9]$.

$$
A_{e}=\frac{G_{r} \lambda^{2}}{4 \pi}, \quad\left[m^{2}\right]
$$

Eqn 2.6

By combining the above equations, the total received power from a reflection off a single object can be written as,

$$
P_{r}=\frac{P_{t} G_{t} G_{r} \sigma \lambda^{2}}{4 \pi\left(4 \pi R^{2}\right)^{2}}
$$

Eqn $2.7[10]$

\subsubsection{Reflected and Transmitted Waves}

The Friis Transmission equation, Eqn 2.7, analysis uses RCS as a measure of the object's reflectivity. The average RCS of an adult male is $1 \mathrm{~m}^{2}$, varying with body position and posture as well as signal frequency [11]. The reason objects, such as a human body, are reflective is due to the dielectric properties of the object's material. The transmitted signals from the radar travel through the air until they reach an object. At the object boundary the propagation medium changes from air to that of the object's material, and thus there is a change in dielectric for the propagating wave. This causes a portion of the signal to reflect off of the boundary at the object, and the rest of the signal to travel through it [12]. The reflected signal, $\mathrm{E}_{\mathrm{r}}$, and transmitted signal, $E_{t}$, are given by

$$
E_{r}=\Gamma E_{i}
$$

Eqn 2.8

$$
E_{t}=\tau E_{i}
$$

Eqn 2.9 
where $\mathrm{E}_{\mathrm{i}}$ is the incident signal, $\Gamma$ is the reflection coefficient, and $\tau$ is the transmission coefficient. The reflection and transmission coefficients are given by,

$$
\begin{gathered}
\Gamma=\frac{\sqrt{\varepsilon_{r 1}}-\sqrt{\varepsilon_{r 2}}}{\sqrt{\varepsilon_{r 1}}+\sqrt{\varepsilon_{r 2}}} \\
\tau=\frac{2 \sqrt{\varepsilon_{r 1}}}{\sqrt{\varepsilon_{r 1}}+\sqrt{\varepsilon_{r 2}}}
\end{gathered}
$$

Eqn 2.10

Eqn 2.11

where $\varepsilon_{\mathrm{r} 1}$ and $\varepsilon_{\mathrm{r} 2}$ are the relative permittivity for the two mediums. The relative permittivity and other properties of different human body tissues are discussed in section 3.3.1.

\subsection{Required System Outputs for Silhouette Capture}

Once known signals are being reflected back off of objects, the radar system can be designed to accomplish the required monitoring goals. The silhouette capture system needs to be able to identify a human body in the monitoring area to track its position over time. In order to do this the system must be able to perform two tasks: find the location of an object based on its reflections, and identify if the object that caused the reflection was a human.

\subsubsection{Object Location}

An object's location can be determined using two pieces of information, the object's distance from the receiver and the angle of the object relative to the receiver. There are various approaches for determining an object's distance from a receiver. These include methods based on time of flight, signal strength, or angle of arrival [13]. Signal strength methods use the known free space path loss attenuation to calculate the objects distance for a constant reflected power. Angle of arrival methods find the object's angle relative to multiple receive antennas and then geometrically calculate the object's distance. Time of flight methods measure the amount of time 
between the transmit signal and the resulting received reflected signal. The relationship between the elapsed time and object distance is given by,

$$
2 d=\frac{v}{t}
$$

Eqn 2.12

Where $\mathrm{v}$ is the velocity of the traveling wave, which is $\mathrm{c}=3 \times 10^{8} \mathrm{~m} / \mathrm{s}$ in air. Twice the object distance is used because the total elapsed time between transmitted and received signals is the time it takes for the signal to propagate out to the object and then back to the receiver, which is twice the object's distance from the receiver.

Determining an object's angle is commonly done by one of two methods. The first is using antenna arrays to determine the angle of arrival of the reflected signal. The antennas in the array are spaced at known distances, and the objects angle can be determined by comparing the received signal phases between the different individual antennas. The second way is to determine an object's angle is using triangulation. By determining the object's distance from two antennas, its location can be calculated as one of two possible solutions. Assuming the system is mounted on a wall of a room facing inwards, the solution behind the system can be discarded, and the objects location is now known. This two-antenna triangulation method is illustrated in Figure

\section{1.}

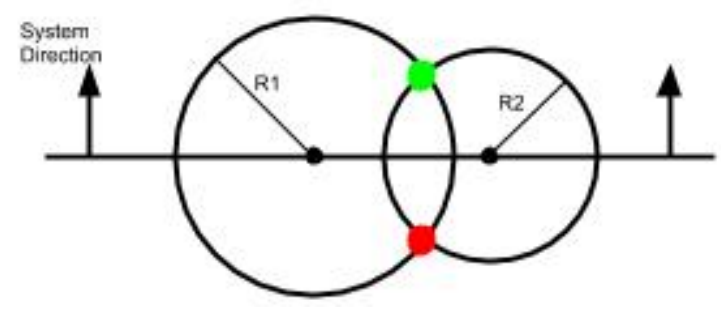

Figure 2.1: Triangulation for Object Location 


\subsubsection{Object Identification}

A common method of detecting humans with radar is by looking for movement. The chest cavity of an average adult male can fluctuate by $0.6 \mathrm{~cm}$ while breathing [6]. Accurate ranging systems can detect this variation in object reflection distance and attribute it to a human. Other systems detect frequency spectral variations that occur in the reflections due to breathing. In either case, by comparing radar returns over time the systems are able to differentiate a person from the surrounding static environment due movement of the body. Current systems that use these methods are discussed in section 3.2.

Another method for determining whether reflections were caused by a human body, is by considering properties of the object's reflectivity. In the case of a human body, a portion of the transmitted signal will reflect back when it reaches the skin. The rest of the signal will travel through the skin until it reaches the next layer. There it will have an additional reflection off of the skin-fat boundary, and this procedure will continue for each layer of the body until what is left of the signal it small enough to be neglected. The reflectivity of each of these individual layers varies over frequency, due to frequency dependent changes in each material's dielectric. By mapping the total radar returns over frequency to the frequency dependency of the individual layer's reflectivity, the reflections can be attributed to coming from a human body. The reflected signals and frequency dependency of human body layers are discussed in section 3.3.1. 


\section{Ultra-Wideband}

An Ultra-Wideband (UWB) system is defined as one that has a bandwidth of $500 \mathrm{MHz}$ or greater, or has a fractional bandwidth of 0.20 or greater [14]. Fractional bandwidth is a measure of how large the system bandwidth is relative to its operating frequency. The UWB bandwidth is bounded by frequency points that are $10 \mathrm{~dB}$ below the maximum radiated emission on either side of the peak radiated power frequency, $\mathrm{f}_{\mathrm{M}}$. The upper boundary is denoted by $\mathrm{f}_{\mathrm{H}}$ and the lower boundary is denoted by $\mathrm{f}_{\mathrm{L}}$. Fractional bandwidth $(\mathrm{FB})$ is defined as,

$$
F B=\frac{2\left(f_{H}-f_{L}\right)}{f_{H}+f_{L}}
$$

Eqn 3.1 [15]

An indoor UWB system, such as the proposed eldercare monitoring radar, must have its bandwidth contained between $3.1 \mathrm{GHz}$ and $10.6 \mathrm{GHz}$ according to the FCC Part 15 specification. The maximum equivalent isotropic radiated power (EIRP) over the passband is $-41.3 \mathrm{dBm}$ over a $1 \mathrm{MHz}$ resolution bandwidth [16]. The allowed UWB spectral density up to $11 \mathrm{GHz}$ according to the FCC specification is shown in Figure 3.1.

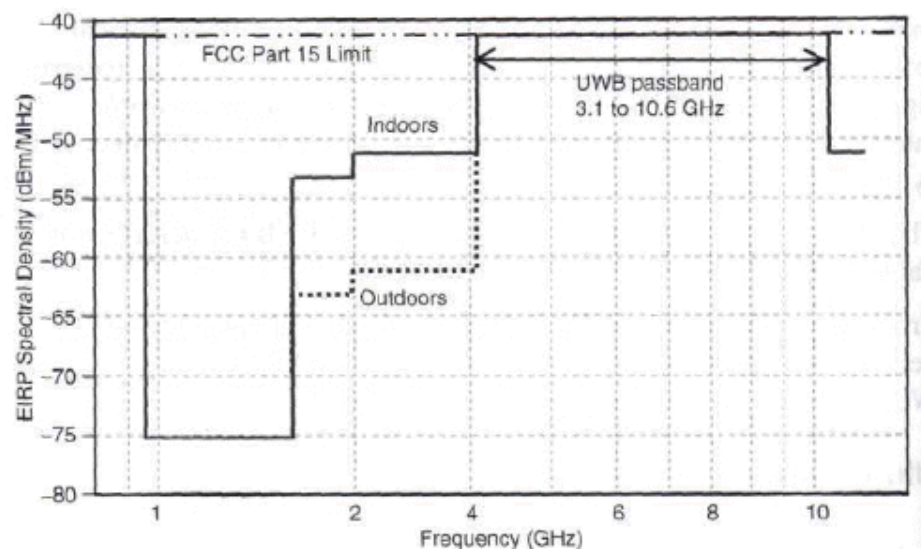

Figure 3.1: UWB EIRP Spectral Density [17] 
The wide frequency bandwidth and low power output of an UWB system differentiates it from classic narrowband systems. Figure 3.2 shows an example power spectrum of a narrowband and UWB system in the frequency domain.

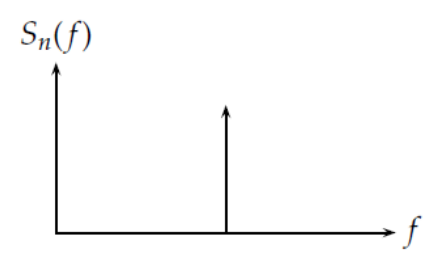

(a) Narrowband

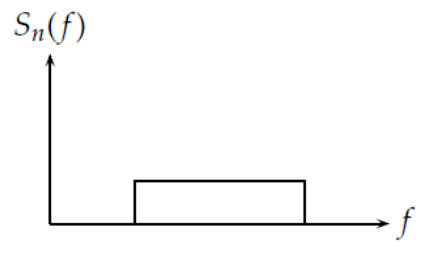

(b) UWB

Figure 3.2: Narrowband and UWB Frequency Spectrum [18]

A signal with a flat power spectrum over all frequencies would appear as an impulse in the time domain. Similarly, an UWB signal appears as a sharp pulse in the time domain due to its wide frequency content. Figure 3.3 shows time domain signals of a narrowband and UWB system.

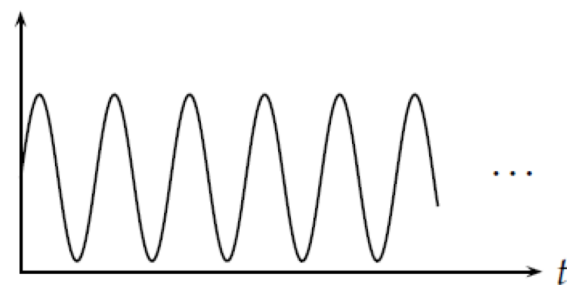

(a) Narrowband

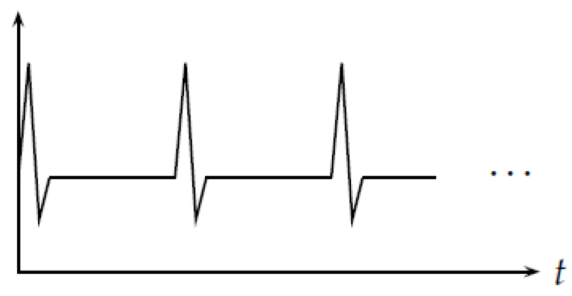

(b) UWB

Figure 3.3: Time Domain Representations of Narrowband and UWB Signals [18]

Ultra-Wideband has many advantages that apply to its use in a radar based monitoring system. This chapter will discuss UWB background, current UWB radars and research, and why UWB was chosen for the proposed system. 


\subsection{Background}

UWB research and development started in the 1960s [19]. Early contributors included Ross and Robbins at the Sperry Rand Corporation, Harmuth at the Catholic University of America, and Paul van Etten at the US Air Force Rome Air Development Center [20]. In 1973 Ross was awarded a US patent on UWB communications [21]. Initial UWB systems were used for ground penetrating radar as well as communication. The micropower impulse radar, invented by T. E. McEwan in 1994, was the first low power UWB radar [22]. In 2002 the FCC released 7.5GHz of unlicensed spectrum for UWB systems, as seen in Figure 3.1.

\subsection{Current UWB Radar and Research}

Current applications for UWB radars include detecting humans trapped in burning buildings, and similar systems are also used for detecting buried avalanche victims. Other UWB radar applications are for close range remote cardiography, as well as other types of medical imaging [6]. In order to develop and improve these systems, recent research has been done in UWB positioning, human detection with UWB, and the UWB frequency response of the human body.

UWB human body reflections have been used to determine target position within $14 \mathrm{~cm}$ from a distance of up to $2 \mathrm{~m}[10]$. By integrating the received reflected pulse signal, signal strength was used to determine the object's distance from a receiver. Four receivers were used to triangulate the target's position and accurately place it. A bench top boundary detection prototype for this project was developed and able to detect the distance of a reflective metal plate within $10 \mathrm{~cm}[18]$.

Positioning a human body amongst background clutter is more involved, because reflections from the background objects have to be differentiated from those of the intended 
target. Current systems determine human position based on movement, either due to a person walking or small fluctuations in the chest cavity due to breathing. This varies the amplitude, frequency, phase, and time of arrival of the reflected wave [6]. By comparing successive measurements over time, a human body can be detected against a static background. Measured data of normalized spectral variations from reflections off a metal sheet, breathing person, and non-breathing person are shown below in Figure 3.4, Figure 3.5, and Figure 3.6 respectively.

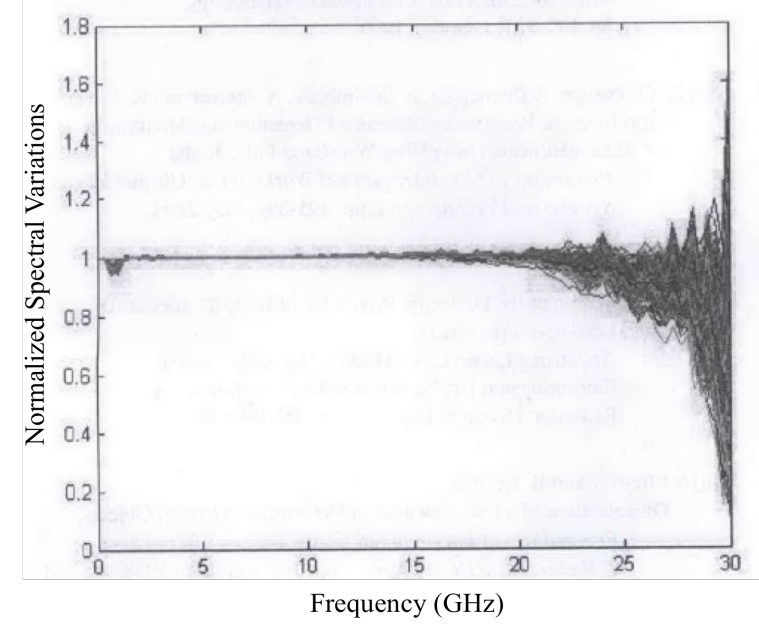

Figure 3.4: Radar Return Spectral Variations from a Metal Sheet [6]

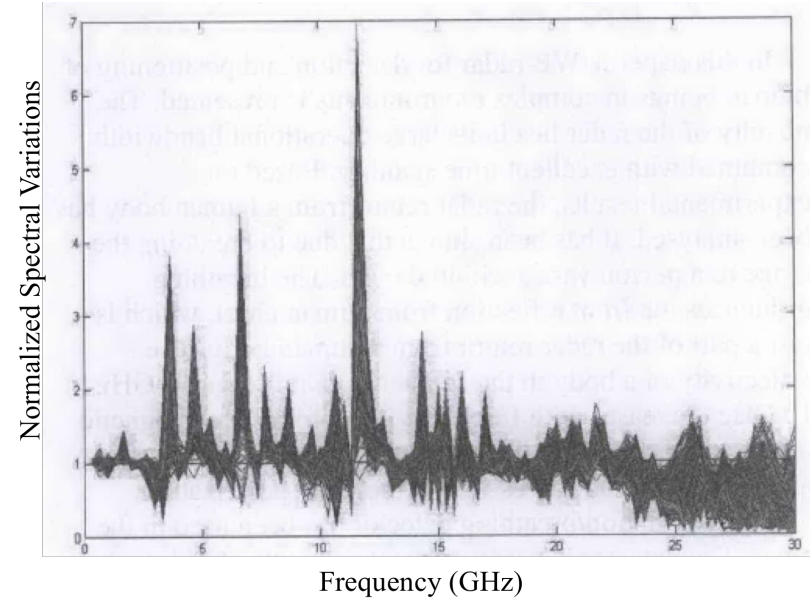

Figure 3.5: Radar Return Spectral Variations from a Breathing Person [6] 


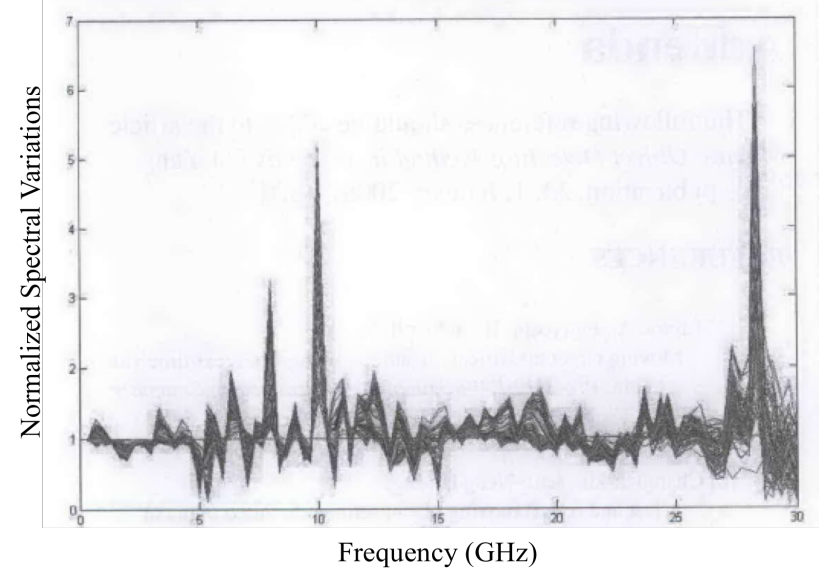

Figure 3.6: Radar Return Spectral Variations from a Non-Breathing Person [6]

Differential measurements like these have been shown to be able to detect human targets though a wall. Testing of brick, concrete block, and rough concrete walls showed an average attenuation of $10 \mathrm{~dB}$ to UWB signals as they pass through [23]. A frequency modulated continuous wave UWB radar was used to measure reflections as a person walked behind the wall. Individual radar return measurements showed large reflections at distances corresponding to the wall, and the person behind it. By comparing measurements over time, the movement and position of the person can be tracked.

\subsection{Human Identification with UWB}

The above systems have shown that UWB reflections off of a human target can be used position that target. When reflections from a human need to be differentiated from those caused by static background clutter, differential measurements are compared over time to detect movement in the body and track the target. However, these systems do not have a method of identifying an individual human in an area with multiple moving and living bodies. The proposed monitoring system needs to have a development path to differentiating the target human from others nearby. 


\subsubsection{Human Body Tissue Properties}

A way to identify a specific target among other moving objects is by analyzing and understanding the individual radar reflections off of that target. When an incoming wave reaches first reaches a human body, the wave changes propagation mediums from air to skin. Because there is a change in the dielectric of these mediums, a portion of the wave will be reflected back and the rest will continue through the skin layer. The portion of the wave that is reflected and transmitted depends on the reflection coefficient, $\Gamma$, and transmission coefficient, $\tau$, which depend on the permittivity of the two mediums. As discussed previously, $\Gamma$ and $\tau$ can be calculated using Eqn 2.10 and Eqn 2.11 respectively.

As the signal propagates through the human body, there will be reflections at each tissue layer transition, the first three of which are shown in Figure 3.7.

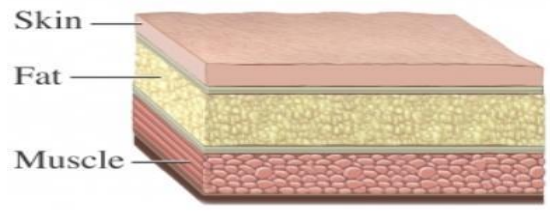

Figure 3.7: Initial Human Body Tissue Layers

Reflections off the skin and fat layer due to an incoming transmitted wave are depicted in Figure 3.8. It shows initial reflections as the transmitted wave moves through the tissue layers, as well as subsequent reflections caused by reflected waves continuing to move between layers. 


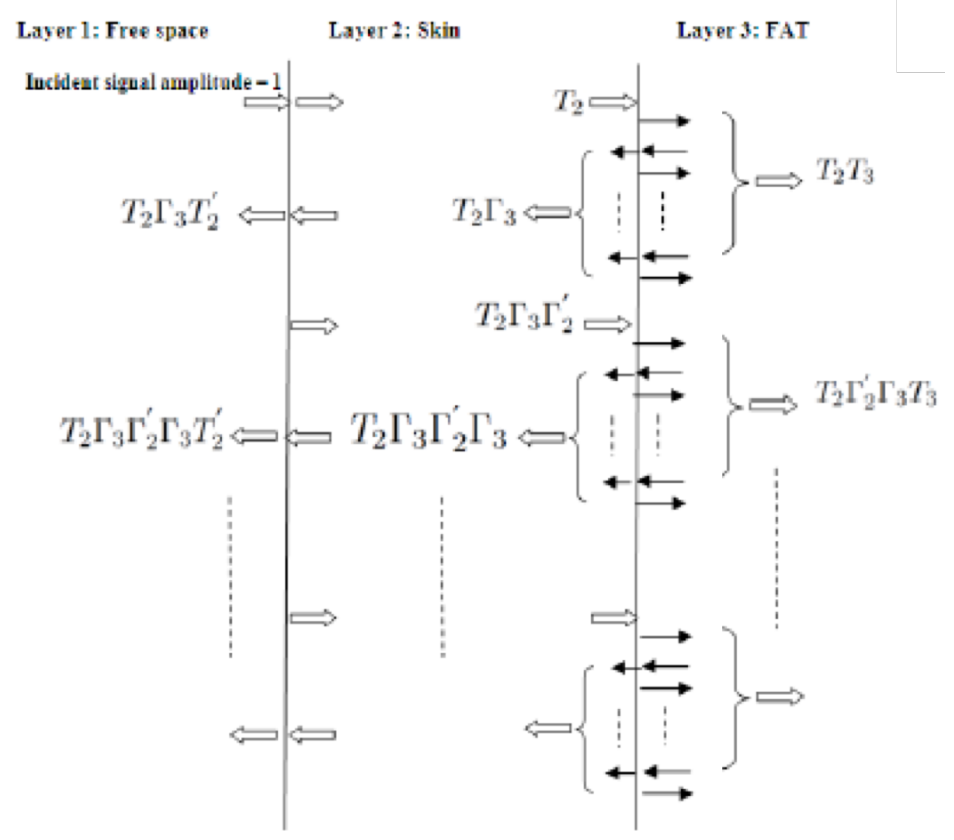

Figure 3.8: Reflected and Transmitted Waves through a Human Body [24]

The reflection at each boundary depends on the permittivity of each of the layers involved. Because the permittivity of body tissues changes with frequency, looking at the magnitude of the reflected wave over a wide frequency range can provide information about the type of material it reflected off. The permittivity across frequency of wet and dry skin, as well as fat and muscle are shown below in Figure 3.9. 


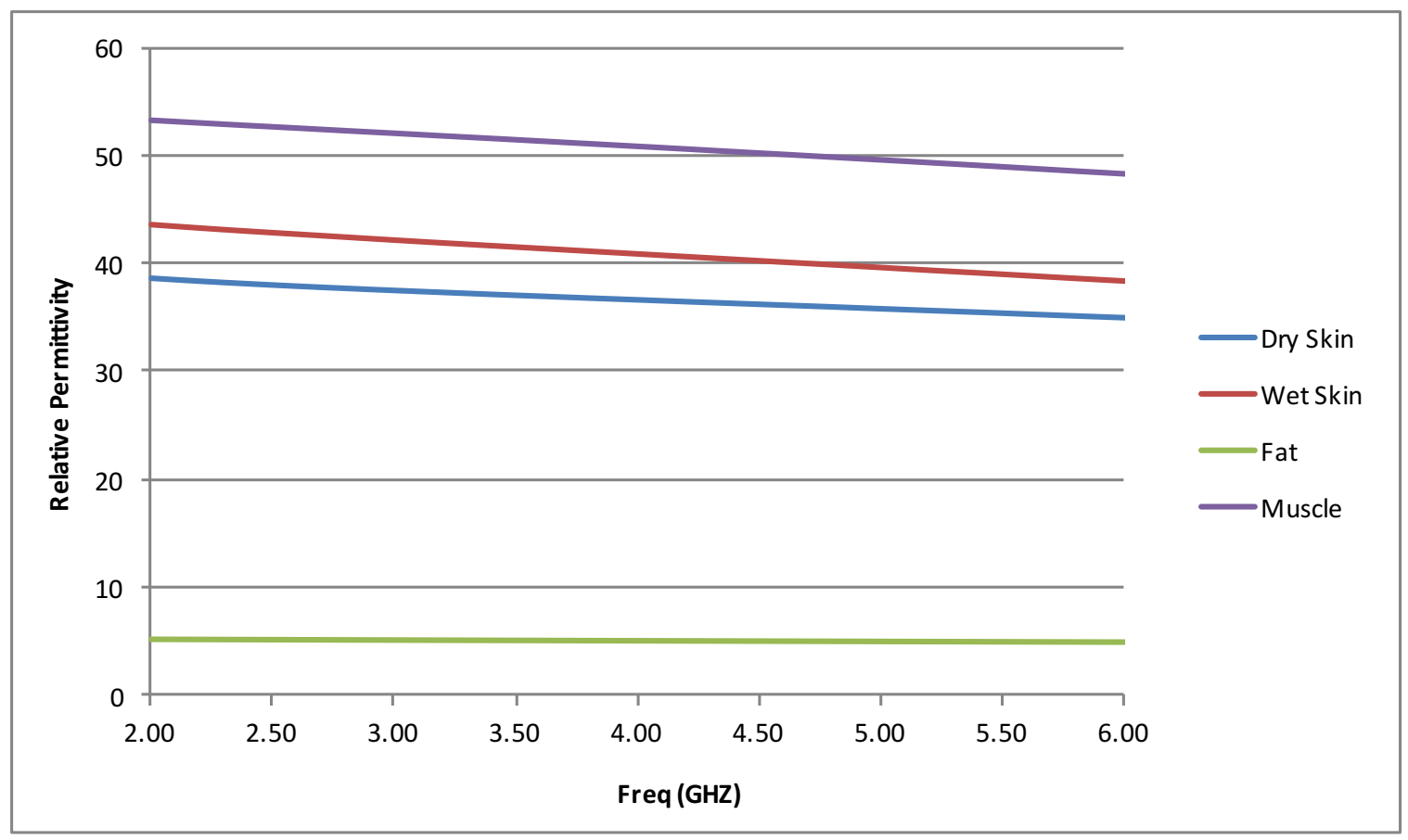

Figure 3.9: Relative Permittivity of Human Body Tissues [25] [26] [27] [28]

The permittivity of a material also affects the propagation velocity of signals travelling through that medium. The relative permittivity of muscle and fat are 50.8 and 5.1 respectively at $4 \mathrm{GHz}$. The corresponding propagation velocities are found by

$$
v=\frac{c}{\sqrt{\varepsilon_{r}}}
$$

Eqn $3.2[12]$

Which gives $\mathrm{v}_{\text {muscle }}=0.42 \times 10^{8} \mathrm{~m} / \mathrm{s}$, and $\mathrm{v}_{\text {fat }}=1.32 \times 10^{8} \mathrm{~m} / \mathrm{s}$. The material thickness and permittivity will affect how long it takes the radar signal to pass through the medium. Therefore, considering the time difference of arrival of different reflections from an object can also provide information about the object's material composition.

The reflected power over frequency can be calculated by combining the concepts above with the reflection and transmission equations in Chapter 2. Specifically, received power from reflections off a human at a distance of $5 \mathrm{~m}$ is of interest for the proposed radar based monitoring system. This procedure is explained in detail in "Uniquely Identifying a Human Among a Clutter 
Using Ultra-Wideband Short Range Radar," a paper in which a simulator was designed to calculate the reflected power off of a human body [29]. Figure 3.10 shows received power for reflections off different body tissue layers from a distance of $5 \mathrm{~m}$.

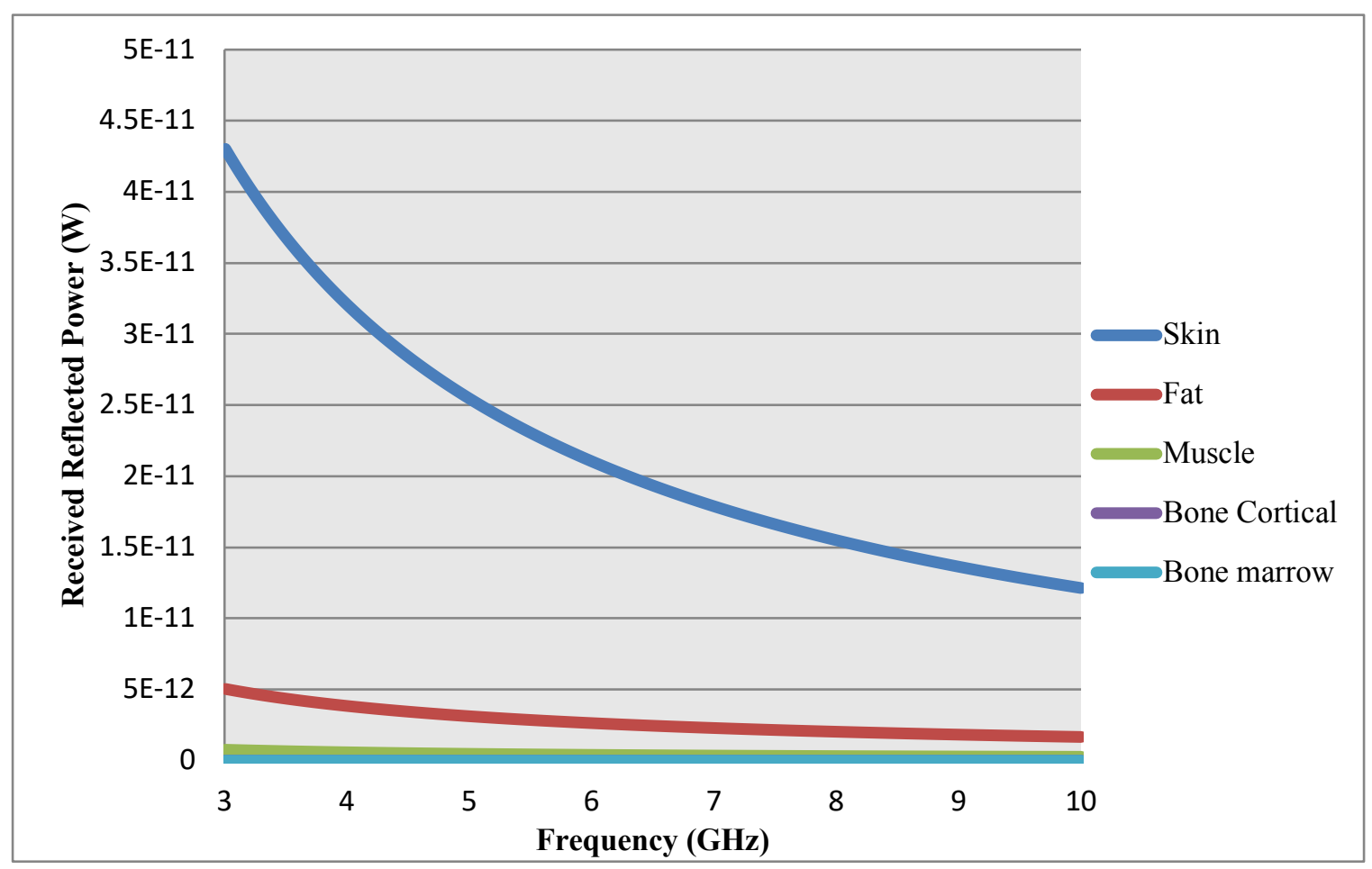

Figure 3.10: Received Reflected Power from a Human Body over Frequency [29]

\subsection{UWB Application to Proposed System}

This section describes many advantages of using UWB radar for detecting human targets. As illustrated by the current UWB radars discussed in section 3.2, many UWB techniques have proved to be capable for human positioning. Now that UWB has been selected for the radar based monitoring system, the operational frequency range and specifics about the transmission method can be determined. 


\subsubsection{UWB Advantages}

The wide bandwidth and low power of UWB radar gives it several advantages over classical narrowband continuous wave radars. The wide bandwidth gives UWB radars a high range resolution, which increases target location precision. The large bandwidth also increases the ability to separate objects from background clutter, as well as increase immunity against multipath interference [24]. UWB signals are also able to pass through obstacles easier to locate obstructed targets [6]. The low power output of UWB radars, $-41.3 \mathrm{dBm} / \mathrm{MHz}$ leads to low energy consumption. It also gives them the ability to operate alongside nearby medical equipment without interference, as the UWB signals appear in the noise floor of the high power narrow band systems.

\subsubsection{System Frequency Spectrum}

UWB technology has been chosen for this radar monitoring system due to its many advantages in the detection and tracking of humans. The FCC specification for indoor UWB systems limits the frequency range between $3.1 \mathrm{GHz}$ and $10.6 \mathrm{GHz}$, as shown previously in Figure 3.1. An optimal subset of this range needs to be used for the system's operational frequency. Because the human body transfer function is not flat over frequency [30], reflected signal to noise ratio (SNR) is expected to vary with system center frequency and bandwidth. This is illustrated in the reflected power calculations depicted in Figure 3.10. The highest SNR for the received reflections of an UWB signal is expected at a center frequency between $4.5-6 \mathrm{GHz}$, and operational bandwidth between 2-2.9GHz [31].

Other considerations include the location of commonly used narrow band frequencies that would interfere with small portions of the UWB operational bandwidth. Figure 3.11 shows 
the FCC frequency spectrum allocation for UWB overlaid with multiple cellular and Wi-Fi frequencies.

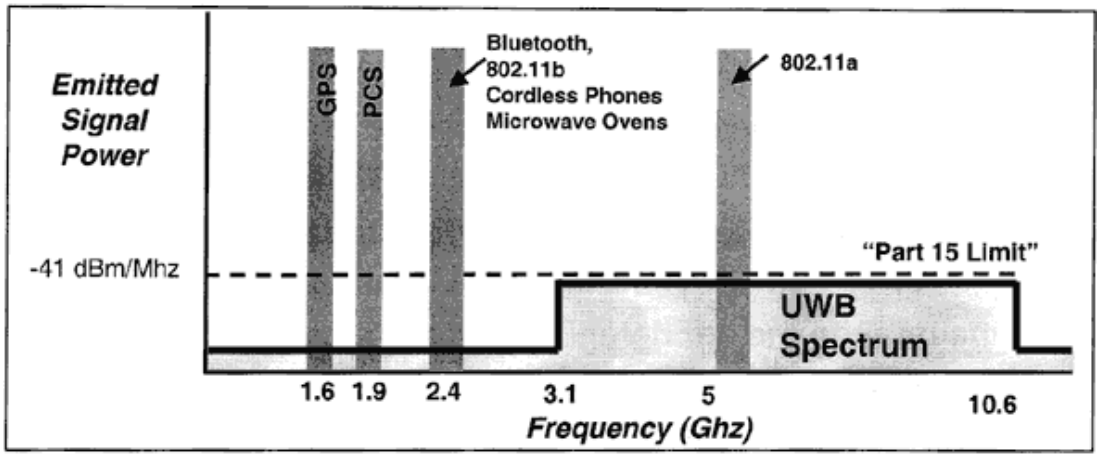

Figure 3.11: FCC Frequency Spectrum Allocation [32]

After taking into the account these considerations, the selected frequency range for the proposed system is $3.1-4.9 \mathrm{GHz}$. This places the system bandwidth in the area of highest reflected power off a human body. The chosen frequency range also does not interfere with $5 \mathrm{GHz}$ Wi-Fi, which would corrupt that portion of the radar's reflected signals. Finally, the bandwidth is wide enough to give sufficient information about the reflecting material, while also allowing manageable component requirements.

\subsubsection{Transmission Method}

Different technologies of UWB radar can be used for positioning human beings among background clutter. Common implementations are video impulse, stepped frequency continuous wave, and frequency modulated continuous wave (FMCW) [33]. The video impulse technique transmits a short pulse, as shown previously in Figure 3.3, and measures the time elapsed before the reflected signal is received. This time difference is used to calculate the objects distance.

The stepped frequency continuous wave and FMCW techniques are similar with the exception that a FMCW system uses an analog swept signal while the stepped frequency uses non-continuous discrete stepped frequency points. A FMCW system transmits a swept frequency 
over time, which propagates out and reflects off the target. The received reflected signal is therefore a swept frequency that is time shifted from the transmit signal by an amount proportional to the target's distance. At any particular point in time the current received signal will have a frequency offset from the current transmit signal. This frequency deviation is proportional to the target's distance.

The continuous wave techniques are preferred over a video impulse system because the received frequency deviations are easier to measure precisely by mixing the transmit and receive signals. On the other hand, the pulse's time of arrival in the video impulse system requires subnanosecond timing circuitry, which can be more complex. A FMCW UWB system was designed and implemented for the silhouette capture subsystem, rather than the stepped frequency continuous wave. This allows the combination of the silhouette capture subsystem with an initial prototype of the boundary detection subsystem. By combining the required hardware for these two subsystems the total system cost, size, and complexity can be reduced.

\subsection{Frequency Modulated Continuous Wave}

The proposed FMCW system is modulated with a sawtooth wave, meaning the frequency sweeps linearly over time from $\mathrm{f}_{\mathrm{L}}$ to $\mathrm{f}_{\mathrm{H}}$. FMCW principles and background for a sawtooth modulated FMCW system are explained in the following sections.

\subsubsection{Time Domain}

The linearly swept frequency over time of the FMCW system repeats periodically, as shown in Figure 3.12. 


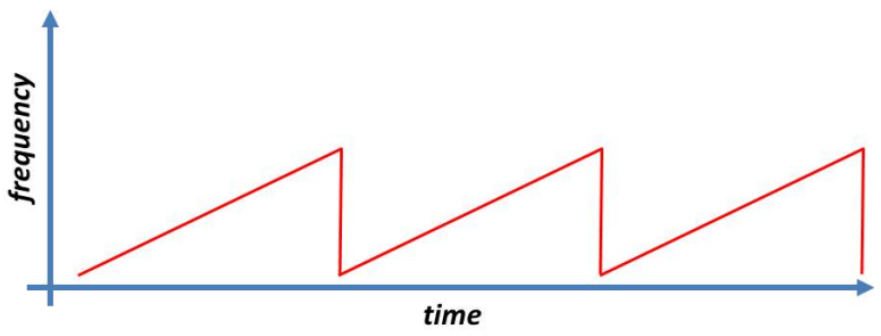

Figure 3.12: FMCW Frequency Sweep [34]

A single period of the signal in the time domain can be represented by,

$$
S(t)=A \cos \left(2 \pi f_{0} t+\pi k t^{2}\right)
$$

Eqn 3.3

Where $\mathrm{A}$ is the signal amplitude, $\mathrm{f}_{0}$ is the start frequency, and $\mathrm{k}$ is the chirp rate, which is discussed in the following section. The signal frequency at any point in time can be found by taking the derivative of the of the phase [35],

$$
f(t)=\frac{1}{2 \pi} \frac{d\left(2 \pi f_{0} t+\pi k t^{2}\right)}{d t}=f_{0}+k t
$$

Eqn 3.4

Using Eqn 3.3, the signal is plotted over time in Figure 3.13.

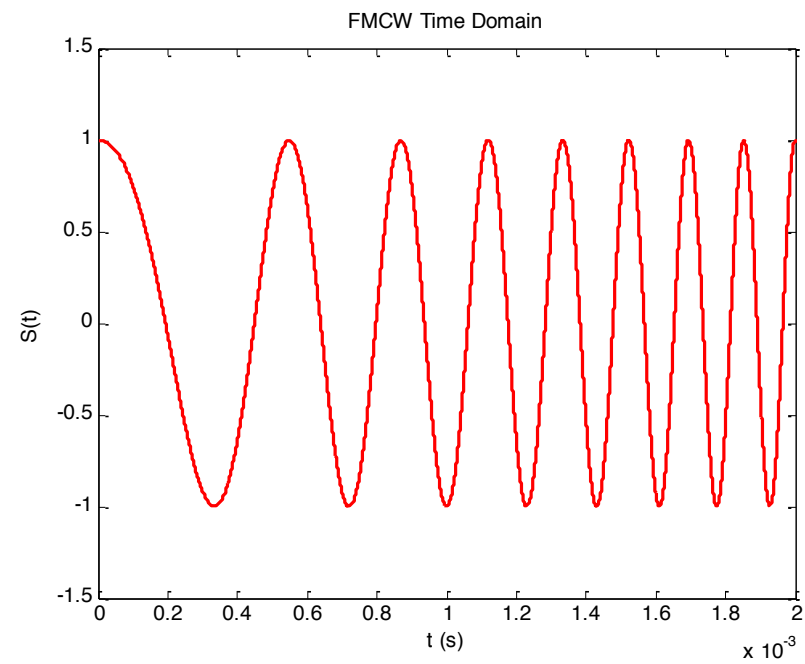

Figure 3.13: Time Domain FMCW Transmit Signal 


\subsubsection{Chirp Rate}

The chirp rate, $\mathrm{k}$, is a measure of the rate of change of the transmit frequency. It is defined as,

$$
k=\frac{B W}{T}
$$

Eqn 3.5

Where BW is the system bandwidth, $\mathrm{f}_{\mathrm{H}}-\mathrm{f}_{\mathrm{L}}$, and $\mathrm{T}$ is the sweep period, which is the time it takes for the system to sweep from $f_{L}$ to $f_{H}$. The transmit signal has reached is max frequency when $t=$ $\mathrm{T}$.

\subsubsection{Time Delay and Frequency Offset}

The transmit signal, as shown in Figure 3.12, propagates through the air until it reaches the target, where it will reflect off and travel back towards the radar system. The received reflection will be delayed in time compared to the transmit signal. The time delay, $t_{d}$ is proportional to the object distance and given by,

$$
t_{d}=\frac{2 R}{v}
$$

Eqn 3.6

Where $\mathrm{R}$ is the object distance, and $\mathrm{v}$ is the propagation velocity which is $\mathrm{c}$ in air. Figure 3.14 shows the transmit and receive signals, as well as the time delay between them.

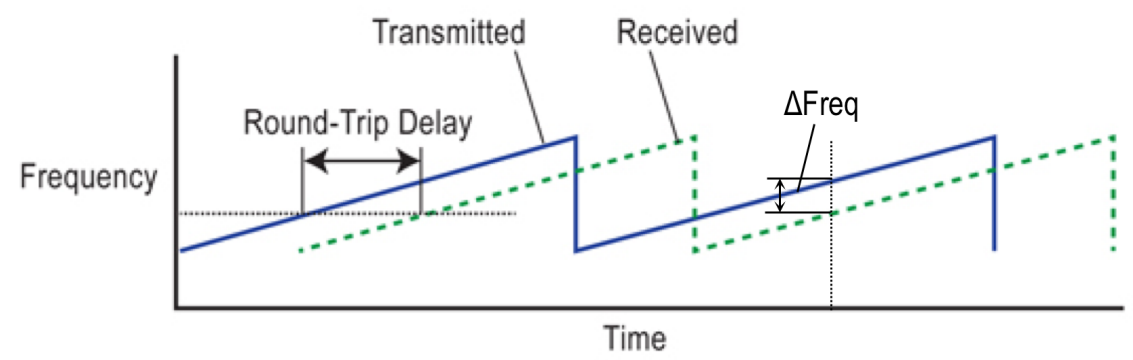

Figure 3.14: Transmit and Received FMCW Signals [36] 
At any instant in time, there is a frequency difference between the current transmit and receive signals. This frequency deviation, also called the beat frequency, $\mathrm{f}_{\mathrm{b}}$, is proportional to the time delay by a factor of the chirp rate.

$$
f_{b}=f_{T X}-f_{R X}=k t_{d}=\frac{B W}{T} t_{d}
$$

Eqn 3.7

The difference between $\mathrm{f}_{\mathrm{TX}}$ and $\mathrm{f}_{\mathrm{RX}}$ can be acquired using a frequency mixer and low pass filtering the higher order mixer products. The beat frequency for two different time delays is shown below in Figure 3.15. The high frequency components are the TX and RX signals, which are seen sweeping from lower to higher frequencies over time. The low frequency envelope is the mixed $f_{T X}-f_{R X}$ frequency, which is proportional to the time delay.
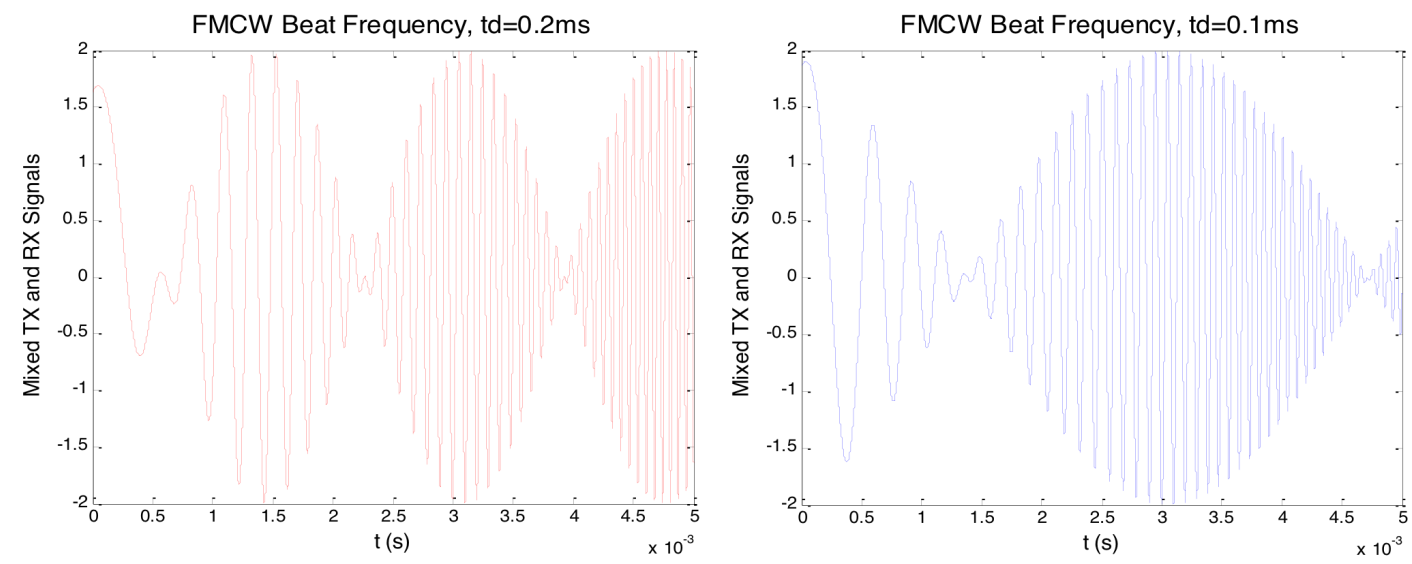

Figure 3.15: Mixed TX and RX Beat Frequencies

\subsubsection{Multiple Targets}

If there are multiple targets in the monitoring area, there will be reflections off each of the targets, with each reflection having a different time delay corresponding to that specific target's distance. In the frequency domain, at the mixer output there will be power at each of the low frequency beat frequencies, while the high frequency received signals and mixer products can be filtered out. 


\section{Proposed Silhouette Capture System RF Front End}

A prototype is designed for the FMCW UWB silhouette capture system RF Front End. Requirements and specifications derivation, system architecture design, component selection, and PCB layout are discussed in this chapter.

\subsection{Performance Requirements}

The silhouette capture system needs to perform two high level functions. First, it must be able to determine the location of an object based on its reflections. Second, it must be able to collect data about the object to determine if it is a human. As discussed in section 3.3 , the reflected power over frequency depends on the object's material composition. In this system, the object location and received power detection needs to work for object distances between $50 \mathrm{~cm}$ and $5 \mathrm{~m}$. The maximum distance was chosen to be approximately the length of an average room. The minimum distance was chosen based off the closest an object should get to the system under normal operation, assuming the system is mounted in the corner of a room.

In order to perform these high-level functions, the transmitter must generate a swept FMCW signal from 3.1-4.9GHz, as discussed in section 3.4.2, which complies with the FCC spectral emission limitations for indoor UWB radars. The receiver must be able to detect low power signals from human reflections $5 \mathrm{~m}$ away from the system, while also detecting reflections from objects as close as $50 \mathrm{~cm}$ without saturating. Based off of these reflections the system must be able to locate the object, using triangulation between two different receive paths and antennas, within a radius of $10 \mathrm{~cm}$. This will allow the system to locate a subject with enough precision to determine its orientation and identify movements in the chest cavity. 


\subsection{System Specifications}

A set of system specifications for the transmitter and receiver can be derived from the above performance requirements. From the indoor UWB radar FCC specification, the transmit power must not exceed $-41.3 \mathrm{dBm} / \mathrm{MHz}$. The out of band spectral emissions must meet the requirements of Figure 3.1. A linearly swept frequency over time from 3.1 to $4.9 \mathrm{GHz}$ is ideal for accurate object detection with FMCW. Non-linearities in the frequency sweep can be compensated for using back end processing if the exact sweep profile is characterized beforehand, as long as the frequency sweep is monotonic.

The absolute worst-case maximum received input signal is $-41.3 \mathrm{dBm}$, which assumes a perfect reflection of the transmit signal without any attenuation. From Figure 3.10 a minimum received input signal of $3.15 \mathrm{pW}$ or $-85 \mathrm{dBm}$ is selected. A $6 \mathrm{~dB}$ minimum SNR requirement sets the required noise floor to $-91 \mathrm{dBm}$. From this the required system noise figure, F, can be determined as a function of the system's noise bandwidth.

$$
F(d B)=\text { noise floor }(\mathrm{dBm})-\text { thermal noise }(\mathrm{dBm})
$$

Eqn 4.1

Where thermal noise depends on the system noise bandwidth in $\mathrm{Hz}, \mathrm{B}$, and is given by,

$$
\text { thermal noise }(\mathrm{dBm})=10 \log \left(\mathrm{kTB} * 10^{3}\right)=-174 \mathrm{dBm} / \mathrm{Hz}
$$

Eqn 4.2

Where $\mathrm{k}=1.38 \times 10^{-23} \mathrm{~J} / \mathrm{K}$, and $\mathrm{T}=290 \mathrm{~K}$.

The maximum system output is set to $3.3 \mathrm{Vpp}$ in order to easily interface with numerous ADCs. The minimum system output is set to $200 \mathrm{mVpp}$ to be easily measured on an oscilloscope for system verification. The largest input signal of $-41.3 \mathrm{dBm}$ reaches $3.3 \mathrm{~V}_{\mathrm{pp}}$ with a gain of $55.65 \mathrm{~dB}$, which becomes the maximum system gain for that input signal level. The minimum 
input signal of $-85 \mathrm{dBm}$ reaches $200 \mathrm{mV}_{\mathrm{pp}}$ with a gain of $75 \mathrm{~dB}$, which becomes the minimum system gain for that input signal level.

A summary of the system specifications for the transmitter and receiver are shown in Table 4.1 and Table 4.2 respectively.

Table 4.1: Tx System Specifications

\begin{tabular}{ll}
\hline Tx Spec & Description \\
\hline $\mathbf{1}$ & Output power less than $-41.3 \mathrm{dBm} / \mathrm{MHz}$ from $3.1-4.9 \mathrm{GHz}$. \\
$\mathbf{2}$ & Output power less than $-51.3 \mathrm{dBm} / \mathrm{MHz}$ above $10.6 \mathrm{GHz}$ and from $1990-3100 \mathrm{MHz}$. \\
$\mathbf{3}$ & Output power less than $-53.3 \mathrm{dBm} / \mathrm{MHz}$ from $1610-1990 \mathrm{MHz}$. \\
$\mathbf{4}$ & Output power less than $-75.3 \mathrm{dBm} / \mathrm{MHz}$ from $960-1610 \mathrm{MHz}$. \\
$\mathbf{5}$ & Linear shaped, monotonic frequency ramp over time from $3.1-4.9 \mathrm{GHz}$. \\
\hline
\end{tabular}

Table 4.2: Rx System Specifications

\begin{tabular}{ll}
\hline Rx Spec & Description \\
\hline $\mathbf{1}$ & Maximum detectable signal of $-41.3 \mathrm{dBm} / \mathrm{MHz}$ between $3.1-4.9 \mathrm{GHz}$. \\
$\mathbf{2}$ & Minimum detectable signal of $-85 \mathrm{dBm} / \mathrm{MHz}$ between $3.1-4.9 \mathrm{GHz}$. \\
$\mathbf{2 . 1}$ & System input noise floor below $-91 \mathrm{dBm} / \mathrm{MHz}$. \\
$\mathbf{3}$ & System output between $200 \mathrm{mV}_{\mathrm{pp}}$ and $3.3 \mathrm{~V}_{\mathrm{pp}}$. \\
$\mathbf{3 . 1}$ & $55.65 \mathrm{~dB}$ gain maximum for an input signal of $-41.3 \mathrm{dBm} / \mathrm{MHz}$ between $3.1-$ \\
$\mathbf{3 . 2}$ & $4.9 \mathrm{GHz}$. \\
& $75 \mathrm{~dB}$ gain minimum for an input signal of $-85 \mathrm{dBm} / \mathrm{MHz}$ between $3.1-4.9 \mathrm{GHz}$.
\end{tabular}




\subsection{System Architecture}

From the system specifications, initial system architectures are designed for the transmitter and receiver. These initial architectures define system operation without yet specifying individual performance of the component(s) for each block. Figure 4.1 and Figure 4.2 show the initial block diagrams for the transmitter and receiver respectively.

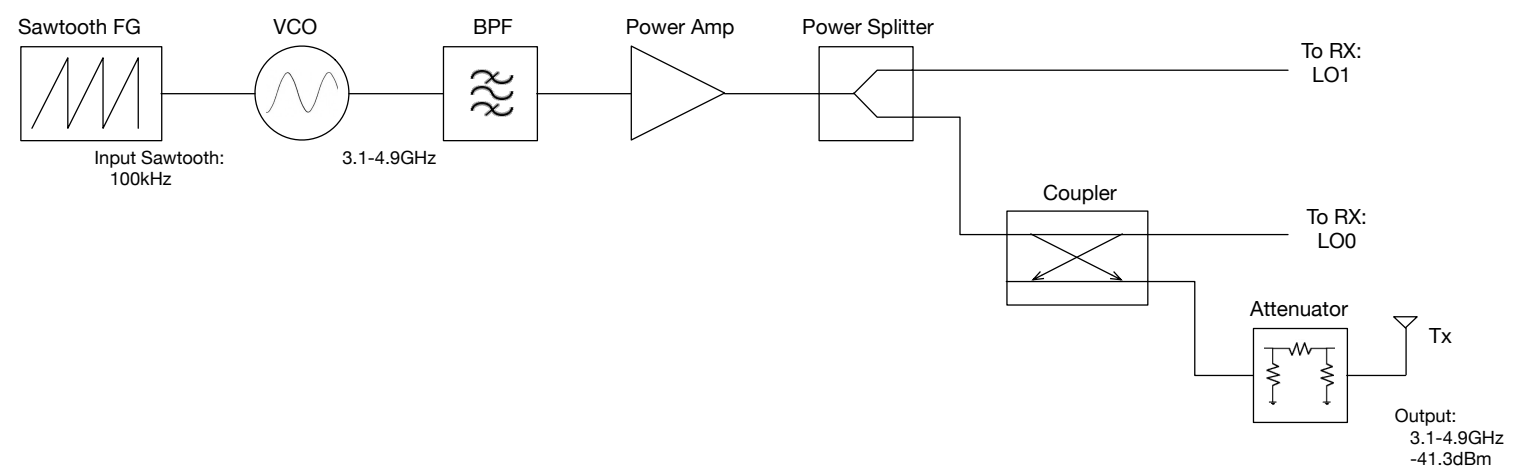

Figure 4.1: Transmitter System Architecture

The FMCW signal is generated from a sawtooth wave that is input into a voltage controlled oscillator (VCO). This creates a tone that is linearly swept in frequency over time. It is then filtered to remove VCO harmonics and amplified high enough to drive the mixer LO port. The signal is then split and fed into a mixer on each of the receive paths, where it is mixed with the received signal in order to find the frequency deviation and thus the object's distance. After the power splitter, the signal is also coupled off and attenuated to meet the transmit power specification, and fed into the TX antenna. 

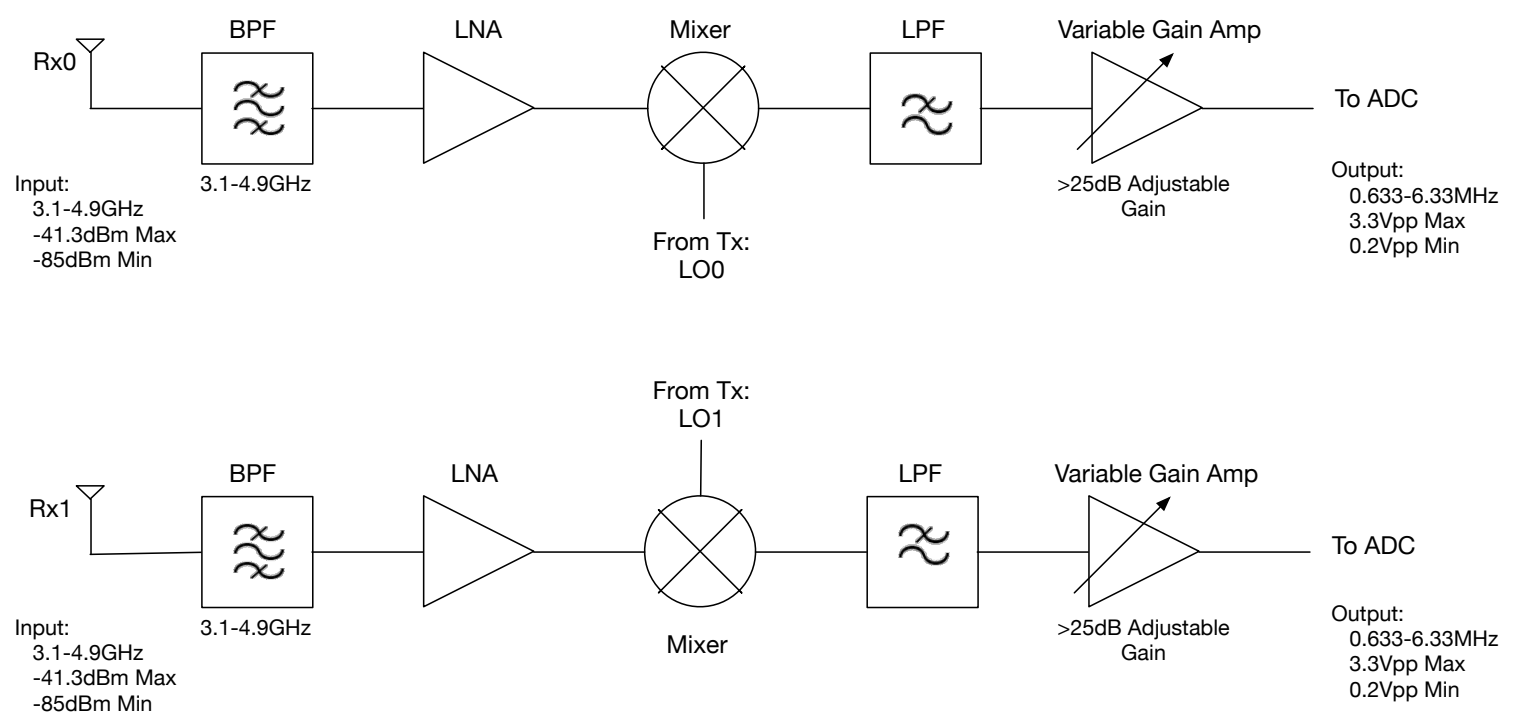

Figure 4.2: Receiver System Architecture

The receive path first passes through a band pass filter to attenuate any high-power blocker signals out of band that would saturate the LNA, such as Wi-Fi or cellular signals. The signal is then amplified and mixed with the current transmit signal. Among the resulting mixer products is the difference between the TX and RX signals, and the low pass filter attenuates the other higher frequency products. The variable gain amplifier (VGA) allows the use of different gain states depending on the input signal level. This gives the system a much higher dynamic range, which is needed to meet the system detection requirements.

After defining overall system operation, a final system parameter needs to be set before individual components can be selected. The period of the sawtooth wave determines how fast the TX signal sweeps from $3.1 \mathrm{GHz}$ to $4.9 \mathrm{GHz}$. And for a given object distance, this sets the difference between the TX and RX frequencies, or the intermediate frequency (IF). Using the minimum and maximum object distances of $50 \mathrm{~cm}$ and $5 \mathrm{~m}$, the round-trip propagation time of the wave can be calculated to be between $3.33 \mathrm{~ns}$ and $33.33 \mathrm{~ns}$. Selecting a sawtooth period of $10 \mu \mathrm{s}$ 
gives IF frequencies between $600 \mathrm{kHz}$ and $6 \mathrm{MHz}$. This frequency range drives the requirements for the LPF and VGA in the receiver.

\subsection{Component Selection}

Now that the system requirements and architecture are defined, specifications for each component are derived and individual components are selected.

\subsubsection{Component Parameter Discussion}

In general, the receiver component requirements are limited by the required system minimum detectable signal; this drives the LNA's noise figure and gain. The insertion loss of the BPF in front of the LNA will also add directly to the cascaded receiver system noise figure. The BPF needs to have attenuation out of band to prevent the receiver from being saturated by any high power out of band jammers. The LNA and mixer need to have high enough linearity to prevent unwanted mixing products that could look like reflections from additional objects. The LPF will help reject the mixing products that land out of the frequency range of interest. The VGA needs to have adjustable gain to vary the cascaded receiver gain between $55 \mathrm{~dB}$ and $75 \mathrm{~dB}$ to meet Rx Specs 3.1 and 3.2.

On the transmit side, the VCO must be able to output a tone from $3.1 \mathrm{GHz}$ to $4.9 \mathrm{GHz}$ with a DC input voltage that could be generated by a bench top Function Generator. The BPF attenuates VCO harmonics and out of band noise to meet Tx Specs 2, 3, and 4. The PA must amplify the signal high enough to drive the LO port of the mixers after it is split to feed both paths. It also must be linear enough to comply with Tx Specs 2, 3, and 4 again. The coupler and attenuator combined must bring the signal low enough to meet the transmit power of $-41.3 \mathrm{dBm}$ per Tx Spec 1. 
Besides the technical performance parameters for each component, additional parameters had to be considered for each part. Component cost was selected to be as low as possible while still being able to meet the system requirements. Component size and package pin out were selected to facilitate manually populating the components on the board, as well as allowing for rework and additional debugging experiments.

\subsubsection{Architecture Modifications}

Taking into account the component parameters discussed above, parts of the architecture had to be modified to meet the system specifications. On the receiver side, in order to meet the required cascaded system noise figure, the LNA block was split into two different components. The first is a traditional low noise amplifier with low noise figure and modest gain. Following that LNA is an additional amplifier with higher noise figure and higher gain. Together these two components are able to provide a low enough cascaded noise figure and high enough cascaded gain to mitigate the noise in the mixer and VGA and meet the required cascaded system noise figure.

Additionally, the LPF on the receiver is split into two different filters to allow for relaxed attenuation requirements on each. The first is a passive LC LPF to attenuate higher order mixer products. The second is an active LPF with low corner frequency, which limits the receiver noise bandwidth and thus sets the baseline for the system noise floor.

On the transmitter side, architecture changes were required to generate the FMCW waveform. Due to component availability and cost constraints, the VCO was selected to output at half the transmit frequency and frequency multiplier is used to reach the target output frequencies. An amplifier is required between the VCO and frequency multiplier to increase the signal enough to drive the passive multiplier. The selected VCO requires an input voltage up to $25 \mathrm{~V}$, which is higher than some bench function generators can output. To allow system 
compatibility with most function generators, a non-inverting op amp with gain of 2 is used at the system input before the VCO.

The original transmitter architecture uses a BPF after the VCO to attenuate out of band noise. To ensure the harmonics from the VCO and frequency multiplier are low enough, a LPF is cascaded with the BPF.

In order to relax the gain requirements on the PA, it is split into two separate components. The first is a gain block amplifier to increase the signal to a manageable level. The second is a PA with enough gain and saturated output power to reach the required output level.

The final architecture change was adding a coupler on each post-splitter path. The second coupler is used solely to keep the path delay on both lines as close as possible. If the delay between the two paths differs, there will be a frequency difference between the LO signal at the two mixers which will lead to errors when triangulating the objects position.

The final transmitter and receiver system block diagrams are pictured below in Figure 4.3 and Figure 4.4.
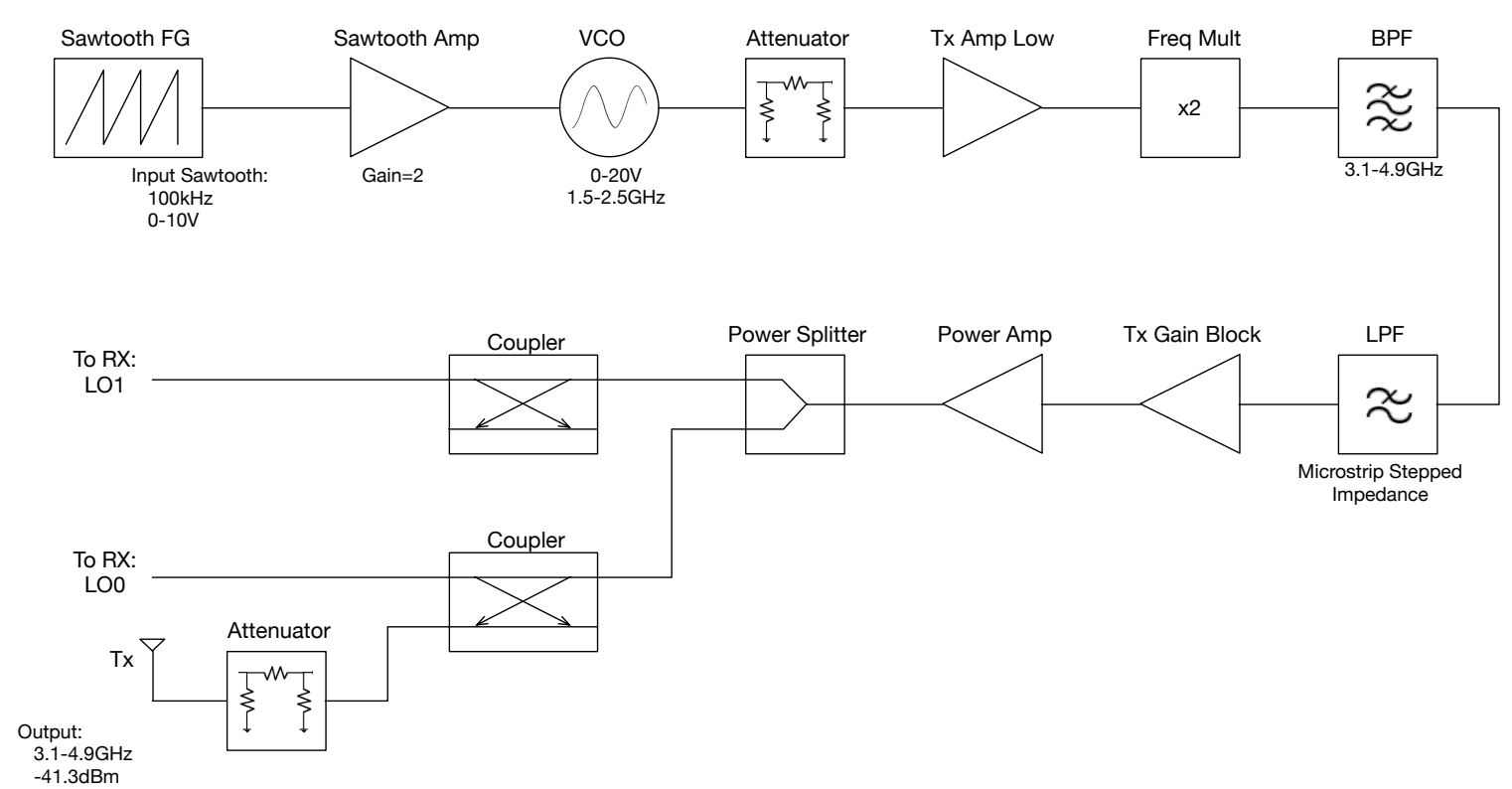

Figure 4.3: Final Transmitter System Block Diagram 

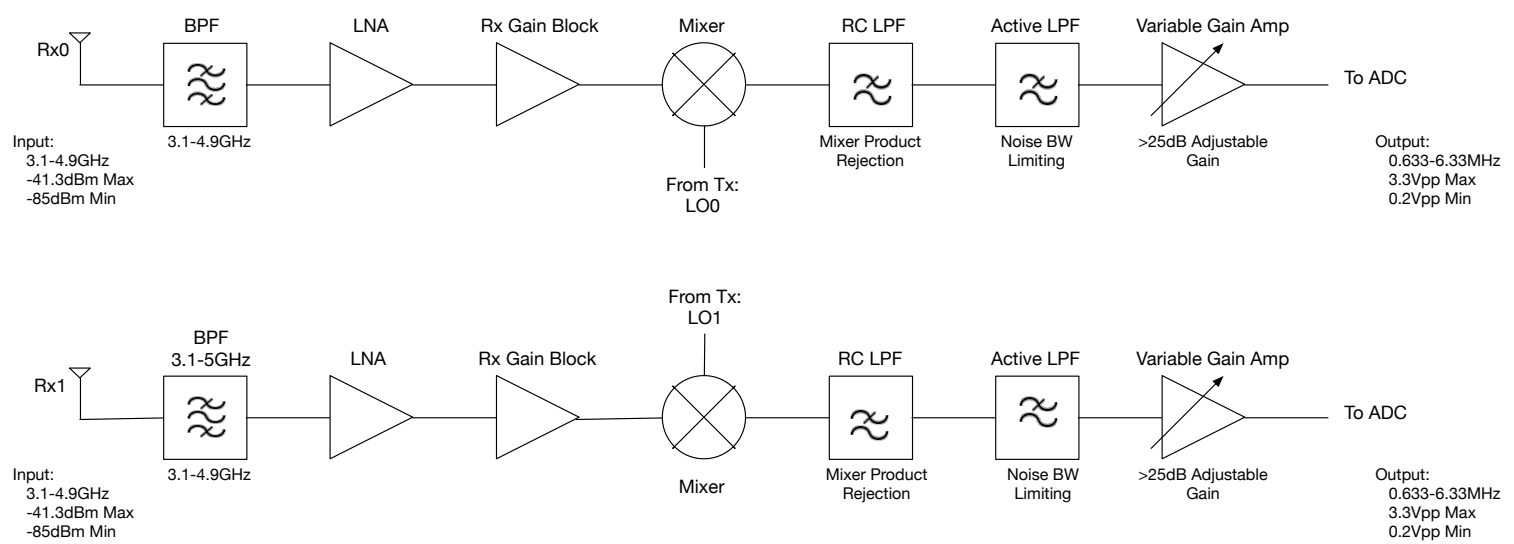

Figure 4.4: Final Receiver System Block Diagram

\subsubsection{Transmitter Components}

Now that the system architecture depicted in the block diagrams of Figure 4.3 and Figure 4.4 is finalized, individual components for each block are selected. Each individual component in the transmitter chain is discussed below. Typical datasheet performance is shown for critical RF parameters that impact system performance. Required external components and voltage rails are also discussed. For manually designed filters and attenuators that don't have datasheets, calculated or simulated performance is shown.

\subsubsection{Sawtooth Amplifier}

A voltage amplifier with gain of two is required to drive the VCO. Texas Instruments LM6211 Op Amp is used in the non-inverting op amp configuration, as depicted in Figure 4.5.

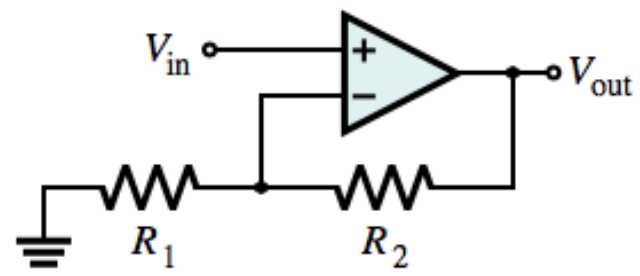

Figure 4.5: Non-Inverting Op Amp [37] 
The gain of a non-inverting op amp is given by,

$$
V_{\text {out }}=\left(1+\frac{R 2}{R 1}\right) V_{\text {in }}
$$

Eqn $4.3[38]$

To achieve a gain of two, $R_{1}$ and $R_{2}$ are chosen to be $1 \mathrm{k} \Omega$. Decoupling capacitors of $100 \mathrm{pF}$ and $0.1 \mu \mathrm{F}$ are used on the $\mathrm{V}^{+}$supply rail to shunt high frequency noise to ground. The LM6211 is capable of operating with a supply voltage between 5-24V. At 24V, typical datasheet performance for slew rate is $5.6 \mathrm{~V} / \mu \mathrm{s}$, and gain bandwidth product is $20 \mathrm{MHz}$ [39].

\subsubsection{Voltage Controlled Oscillator}

The swept FMCW waveform is generated with a voltage controlled oscillator. The sawtooth waveform is input to a Mini-Circuits ROS-2700-1819+, which is capable of outputting 1300-2700MHz. The frequency tuning voltage characteristic in Figure 4.6 shows the sawtooth $\mathrm{V}_{\min }$ and $\mathrm{V}_{\max }$ will need to be $4 \mathrm{~V}$ and $15.5 \mathrm{~V}$ respectively for the required FMCW frequencies.

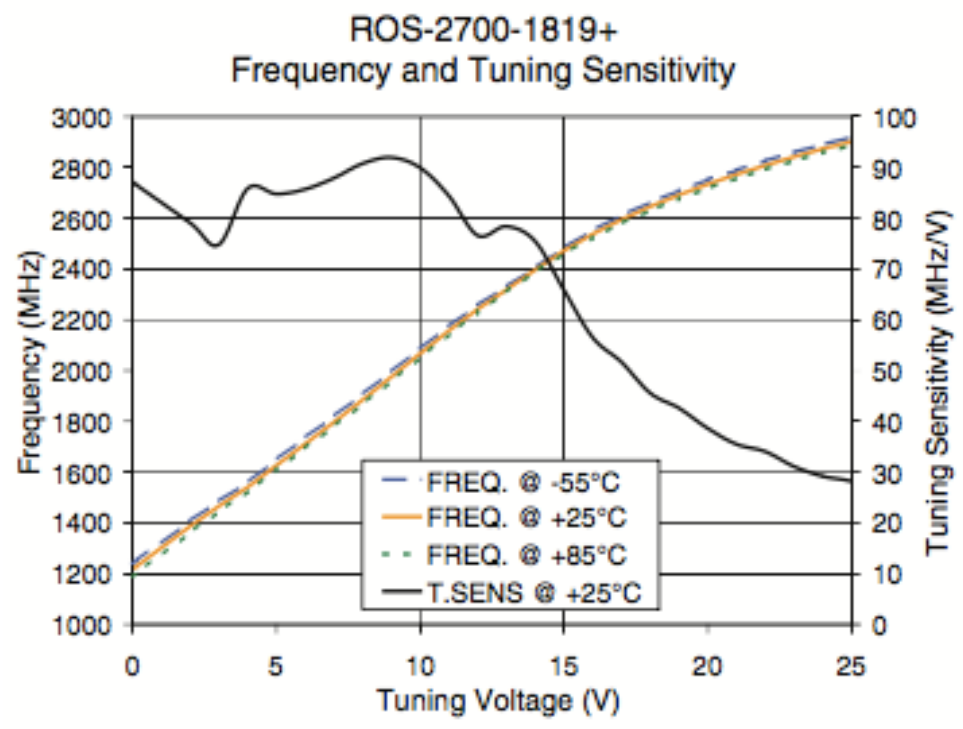

Figure 4.6: ROS-2700-1819+ Datasheet Frequency Tuning Voltage [40]

The fundamental output power as well as the second, third, and fourth harmonic levels are shown versus tuning voltage below in Figure 4.7. 

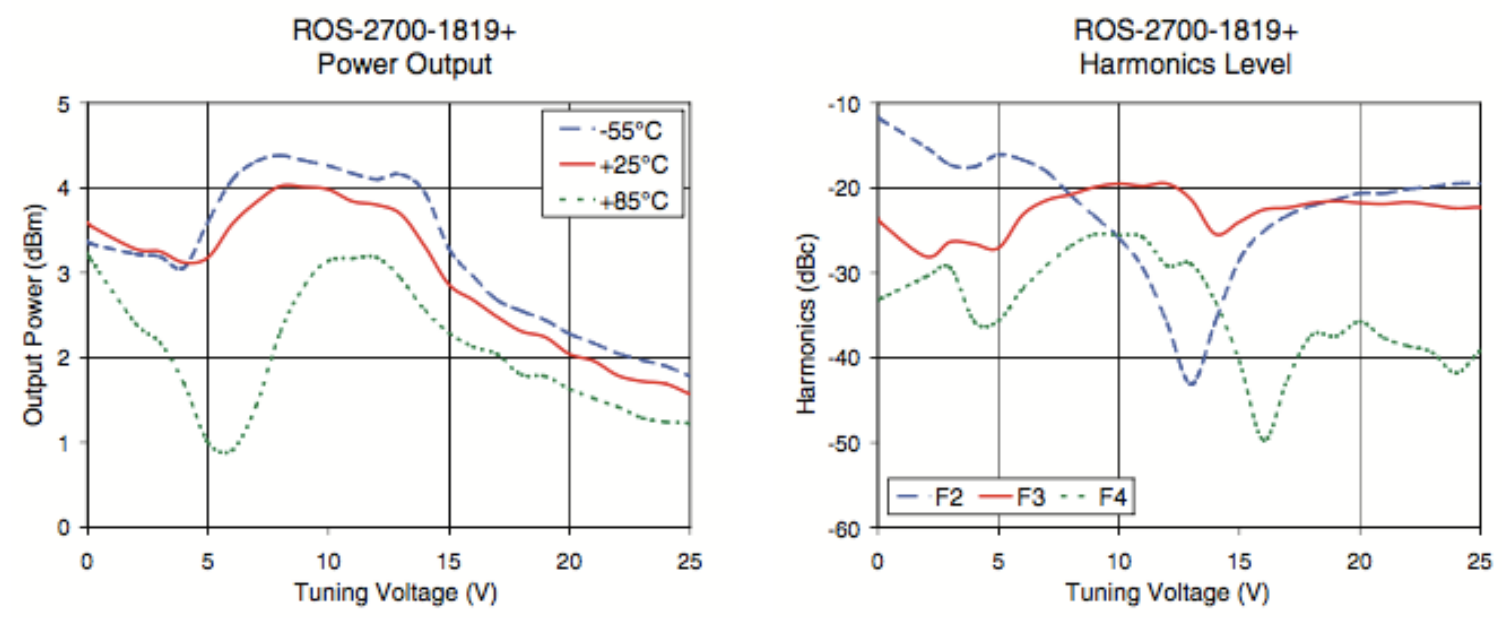

Figure 4.7: ROS-2700-1819+ Datasheet Pout and Harmonic Levels [40]

\subsubsection{Low Frequency Tx Amplifier and Input Attenuator}

A frequency multiplier is used to double the VCO output, which operates at half the required system frequency. In order to drive the frequency multiplier, an amplifier is used to boost the VCO output. Skyworks SKY67014-396LF operates from 1.5-3GHz off of a 3.3V rail. It uses external $50 \Omega$ input and output matching, as well as a bias network and decoupling capacitors. An external resistor, selected at $2.2 \mathrm{k} \Omega$, sets the amplifier's quiescent current at $18 \mathrm{~mA}$. Datasheet specifications are shown below in Table 4.3. 
Table 4.3: SKY67014-396LF Datasheet Specifications, Icq=18mA [41]

\begin{tabular}{|c|c|c|c|c|c|c|}
\hline Parameter & Symbol & Test Condition & Min & Typical & Max & Units \\
\hline \multicolumn{7}{|l|}{ RF Specifications } \\
\hline Noise Figure & NF & $\begin{array}{l}\text { Evaluation Board losses } \\
\text { included }\end{array}$ & & 0.85 & 1.05 & $\mathrm{~dB}$ \\
\hline Small signal gain & |S21| & & 12 & 13 & & $\mathrm{~dB}$ \\
\hline Input return loss & $|\mathrm{S} 11|$ & & 12 & 15 & & $\mathrm{~dB}$ \\
\hline Output return loss & $|\mathrm{S} 22|$ & & 8 & 11 & & $\mathrm{~dB}$ \\
\hline Reverse isolation & $|\mathrm{S} 12|$ & & 20 & 23 & & $\mathrm{~dB}$ \\
\hline $3^{\text {rd }}$ Order Input Intercept Point & ॥P3 & $\begin{array}{l}\Delta f=1 \mathrm{MHz} \\
P_{N}=-20 \mathrm{dBm} / \text { tone }\end{array}$ & +12 & +15 & & $\mathrm{dBm}$ \\
\hline $3^{\text {rd }}$ Order Output Intercept Point & OIP3 & $\begin{array}{l}\Delta f=1 \mathrm{MHz}, \\
\mathrm{PN}_{\mathrm{N}}=-20 \mathrm{dBm} / \text { tone }\end{array}$ & +25 & +28 & & $\mathrm{dBm}$ \\
\hline $1 \mathrm{~dB}$ Input Compression Point & IP1dB & & +1.5 & +3.5 & & $\mathrm{dBm}$ \\
\hline $1 \mathrm{~dB}$ Output Compression Point & OP1dB & & +13.5 & +15.5 & & $\mathrm{dBm}$ \\
\hline \multicolumn{7}{|l|}{ DC Specifications } \\
\hline Supply voltage & Vod & & 3.0 & 3.3 & 5.5 & V \\
\hline Supply current & loo & Set with external resistor & & 18 & & $\mathrm{~mA}$ \\
\hline
\end{tabular}

The VCO output level plus amplifier gain is $4.5 \mathrm{~dB}$ higher than the frequency mixer can handle. Therefore, a $50 \Omega 4.5 \mathrm{~dB}$ PI pad attenuator is used at the amplifier input, as shown in

Figure 4.8, where $\mathrm{R}_{1}=\mathrm{R}_{3}$.

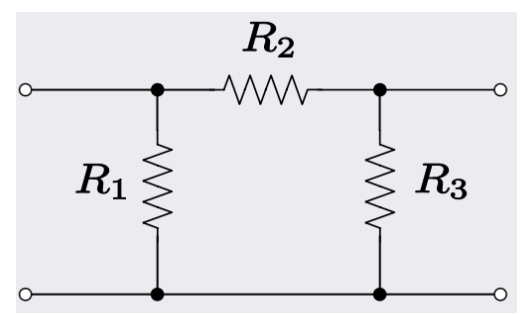

Figure 4.8: PI Attenuator

The input and output impedance are calculated in Eqn 4.4, and attenuation in Eqn 4.5.

$$
\begin{gathered}
Z_{\text {in }}=Z_{\text {out }}=\left(R_{3} \| 50+R_{2}\right) \| R_{1} \\
\text { Attenuation }(d B)=-10 \log \left(\frac{V_{o}}{V_{i}}\right)=-10 \log \left(\frac{R_{3} \| 50}{R_{2}+R_{3} \| 50}\right)
\end{gathered}
$$

Eqn 4.4

Eqn 4.5

Choosing $\mathrm{R}_{1}=\mathrm{R}_{3}=196 \Omega$, and $\mathrm{R}_{2}=27 \Omega$ gives $4.89 \mathrm{~dB}$ attenuation and $49.84 \Omega$ input and output impedances. 


\subsubsection{Frequency Multiplier}

A Mini-Circuits KSX2-722+ x2 Frequency Multiplier is used to double the VCO output frequency to the target system operating frequencies. It is able to output $2600-7200 \mathrm{MHz}$ from a 9-13dBm input signal with typical conversion loss of $11.5 \mathrm{~dB}$ [42]. Figure 4.9 below shows typical conversion loss and harmonic suppression versus frequency input levels of 9 and $13 \mathrm{dBm}$.
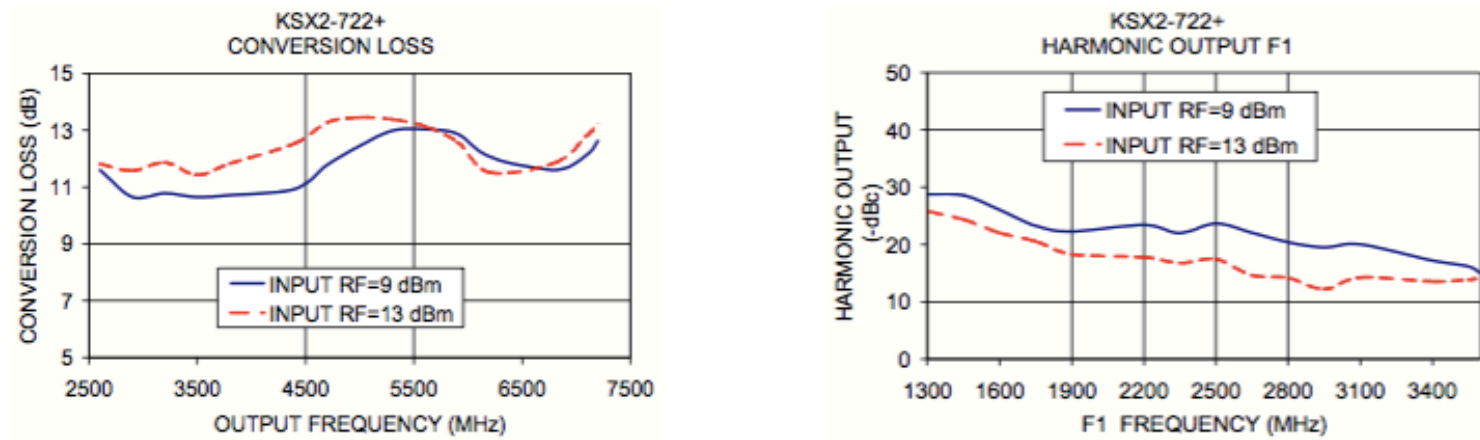

KSX2-722+
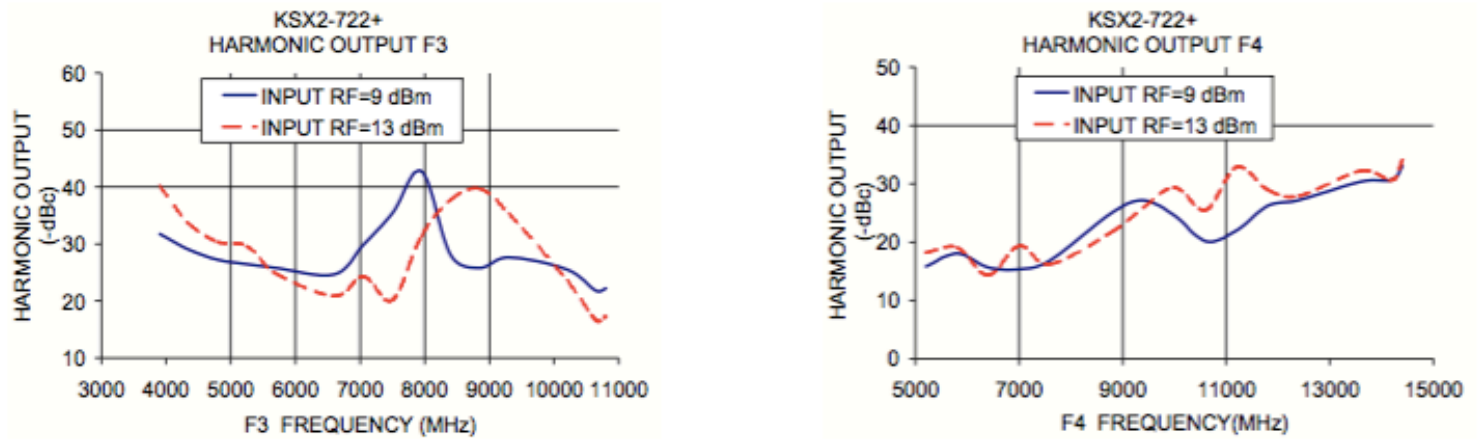

Figure 4.9: KSX2-722+ Typical Datasheet Performance [42]

\subsubsection{Band Pass Filter}

TDK UWB Band Pass Filter DEA453960BT-3002B1 is used to attenuate unwanted out of band products from the VCO and frequency multiplier. It passes the in-band frequencies that the system operates at, and attenuates harmonics and other out of band frequencies generated by higher order mixing products. The datasheet specifications are shown below in Table 4.4. 
Table 4.4: TDK UWB BPF DEA453960BT-3002B1 Datasheet Specifications [43]

\begin{tabular}{|l|l|c|c|c|c|}
\hline \multicolumn{1}{|c|}{ PARAMETER } & Frequency $[\mathrm{MHz}]$ & MIN. & TYP. & MAX. & UNIT \\
\hline Insertion loss $\left(\mathrm{T}=25^{\circ} \mathrm{C}\right)$ & $3168-4752$ & & 1.7 & 1.9 & $\mathrm{~dB}$ \\
\hline Insertion loss $\left(\mathrm{T}=-30^{\circ} \mathrm{C}\right.$ to $\left.85^{\circ} \mathrm{C}\right)$ & $3168-4752$ & & & 2.2 & $\mathrm{~dB}$ \\
\hline Input Return loss & $3168-4752$ & 10 & & & $\mathrm{~dB}$ \\
\hline Attenuation & $\mathrm{DC}-2200$ & 30 & & & $\mathrm{~dB}$ \\
\hline Attenuation & $2400-2500$ & 26 & & & $\mathrm{~dB}$ \\
\hline Attenuation & $5150-5300$ & 23 & & & $\mathrm{~dB}$ \\
\hline Attenuation & $5300-5900$ & 25 & & & $\mathrm{~dB}$ \\
\hline Attenuation & $6336-9504$ & 10 & & & $\mathrm{~dB}$ \\
\hline
\end{tabular}

\subsubsection{Microstrip Stepped Impedance Low Pass Filter}

A low pass filter is cascaded with the above BPF to increase harmonic attenuation. A stepped impedance microstrip filter design is selected due to the high operating frequencies, which would require impractical values of discrete capacitors and inductors. The filter design is a $7^{\text {th }}$ order stepped impedance Butterworth LPF with $5.5 \mathrm{GHz}$ cutoff frequency. After the initial microstrip line dimensions are calculated from the filter coefficient tables, the dimensions are simulated and optimized in ADS to improve performance. Figure 4.10 and Figure 4.11 below show the ADS simulation schematic and the simulation results after optimization.

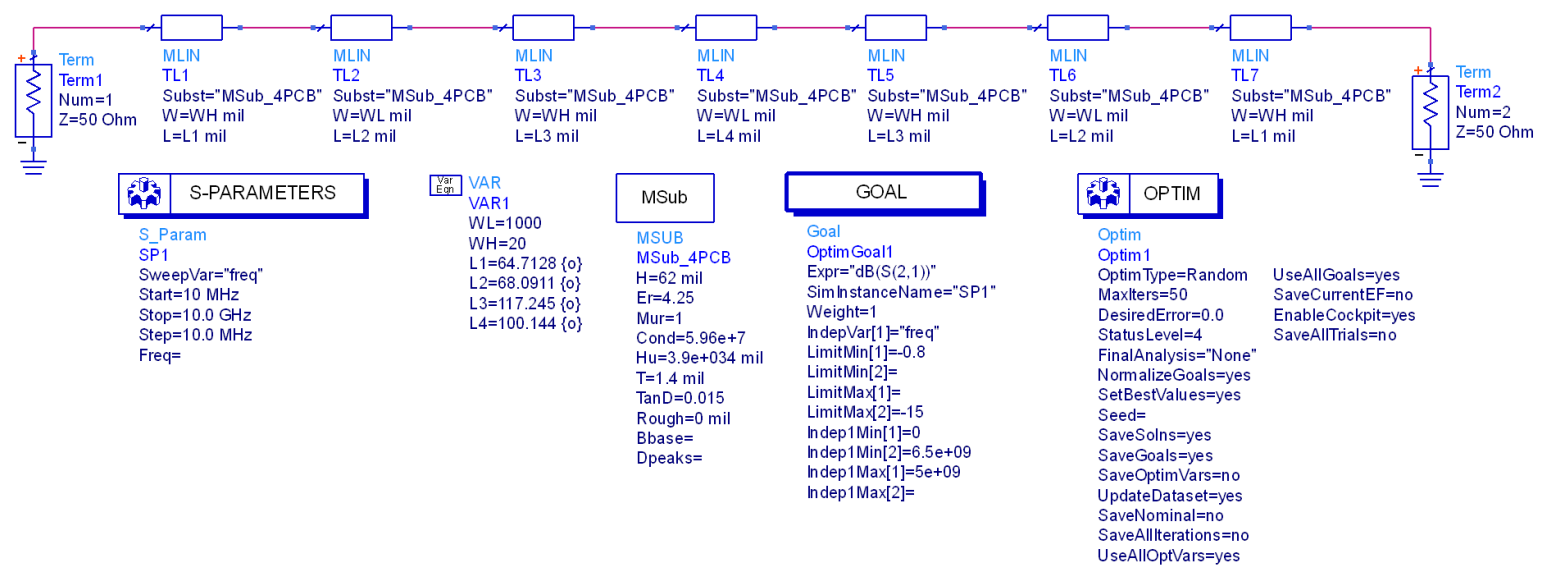

Figure 4.10: Stepped Impedance LPF ADS Simulation 


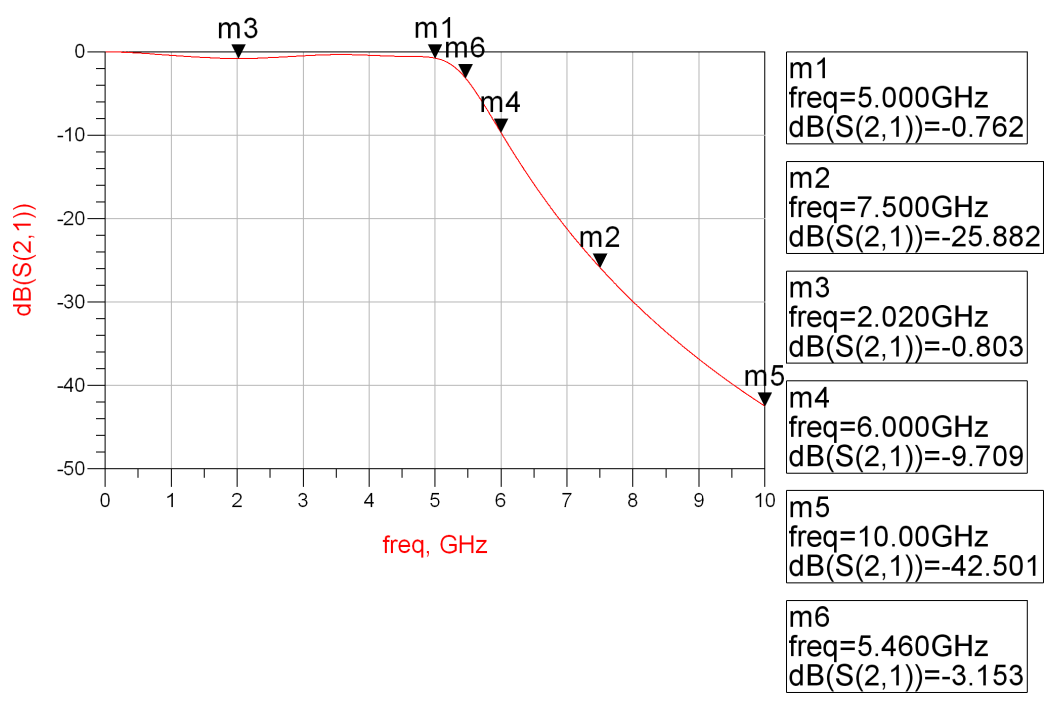

Figure 4.11: Stepped Impedance LPF Simulation Results

\subsubsection{Tx Gain Block Amplifier}

The first amplifier used to increase the transmit signal high enough to drive the LO port of the mixer is an Analog Devices ADL5541, which is a 50-6000MHz RF gain block with a fixed gain of $15 \mathrm{~dB}$. The input and output are internally matched to $50 \Omega$, and it has an integrated bias control circuit. Operating off a single 5V supply, at $900 \mathrm{MHz}$ it has $\mathrm{P} 1 \mathrm{~dB}_{\text {out }}$ of $19.7 \mathrm{dBm}$ and IP $3_{\text {out }}$ of $40 \mathrm{dBm}$ [44]. It requires external input and output AC coupling capacitors, power supply decoupling capacitors, and an external bias inductor. Typical datasheet performance for IP $3_{\text {out, }}$ $\mathrm{P} 1 \mathrm{~dB}_{\text {out, }}$, gain, and noise figure are shown versus frequency below in Figure 4.12.

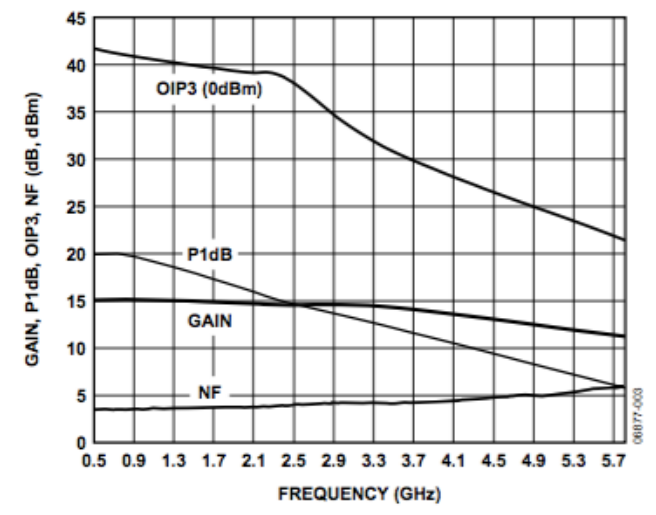

Figure 4.12: ADL5541 Typical Datasheet Performance [44] 


\subsubsection{Tx Power Amplifier}

After the Tx Gain block, the signal is amplified high enough to drive the LO port of the mixers with an Avago MGA-83563. This power amplifier can operate from $0.5-6 \mathrm{GHz}$ with a $+22 \mathrm{dBm} \mathrm{P}_{\mathrm{SAT}}$ on a $3 \mathrm{~V}$ rail [45]. It uses an external input and output match as well as a bias inductor and power supply decoupling capacitors. Typical datasheet gain, $\mathrm{P} 1 \mathrm{~dB}$, and $\mathrm{P}_{\mathrm{SAT}}$ are shown versus frequency below in Figure 4.13. Figure 4.14 shows $\mathrm{P}_{\text {out }}$ and IP3 versus input power.
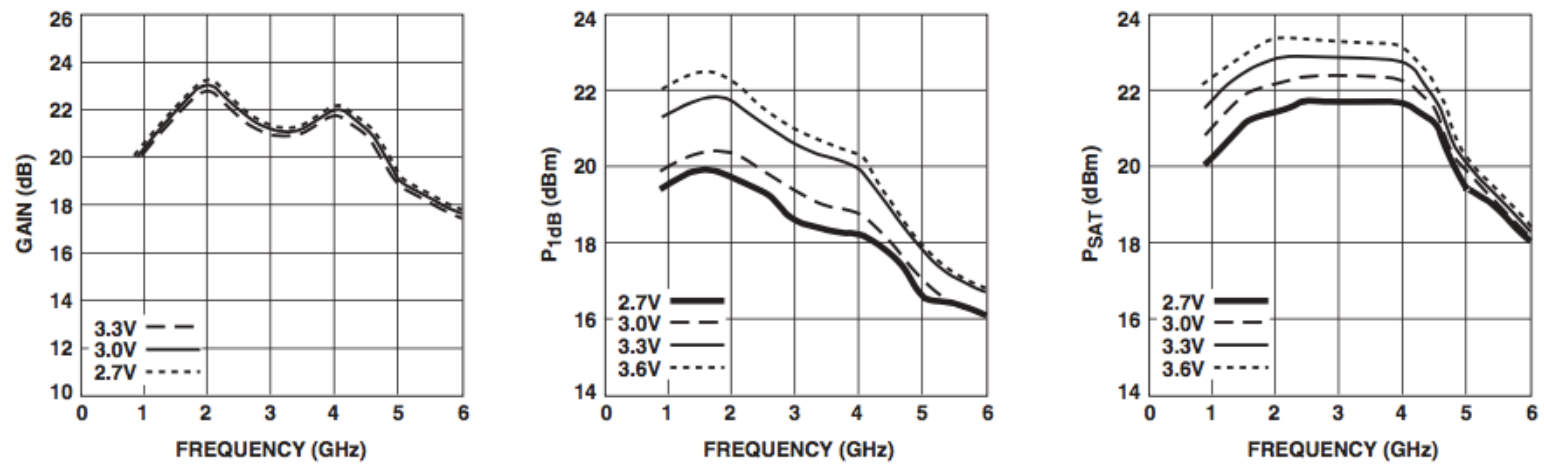

Figure 4.13: MGA-83563 Typical Datasheet Performance vs. Frequency [45]
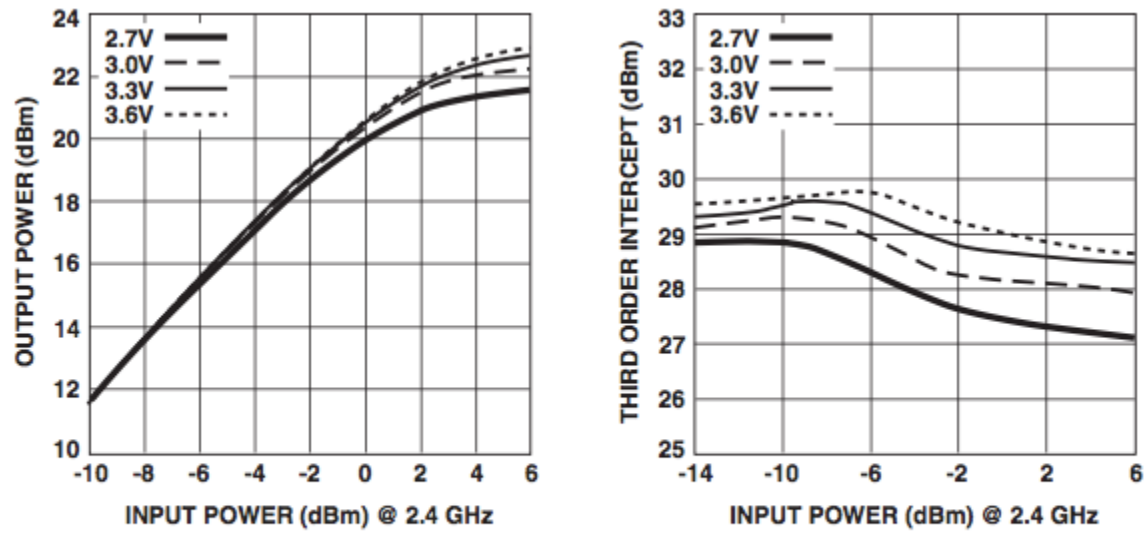

Figure 4.14: MGA-83563 Typical Datasheet Performance vs. Input Power [45]

\subsubsection{Power Splitter}

A Mini-Circuits GP2X+ Power Splitter is used to split the transmit signal to feed the LO ports of the mixer on each receiver chain. It operates from $2900-6200 \mathrm{MHz}$ up to a maximum 
input signal of $1.5 \mathrm{~W}$. The datasheet specifications are shown below in Table 4.5, and typical total loss and isolation are shown versus frequency in Figure 4.15.

Table 4.5: GP2X+ Datasheet Specifications [46]

\begin{tabular}{|c|c|c|c|c|c|c|}
\hline $\begin{array}{l}\text { FREQ. } \\
\text { RANGE } \\
(\mathrm{MHz})\end{array}$ & $\underset{\text { (dB) }}{\text { ISOLATION }}$ & $\begin{array}{l}\text { INSERTION LOSS* } \\
\text { (dB) } \\
\text { ABOVE } 3.0 \mathrm{~dB}\end{array}$ & $\begin{array}{c}\text { PHASE } \\
\text { UNBALANCE } \\
\text { (Degrees) }\end{array}$ & $\begin{array}{l}\text { AMPLITUDE } \\
\text { UNBALANCE } \\
\text { (dB) }\end{array}$ & & \\
\hline$t_{L}-f_{U}$ & Typ. Min. & Typ. Max. & & & $\begin{array}{c}\text { Port } \\
\text { S }\end{array}$ & $\begin{array}{c}\text { Ports } \\
1,2\end{array}$ \\
\hline $2900-6200$ & 15 & 1.5 & 9.0 & 0.3 & 1.2 & 1.2 \\
\hline
\end{tabular}
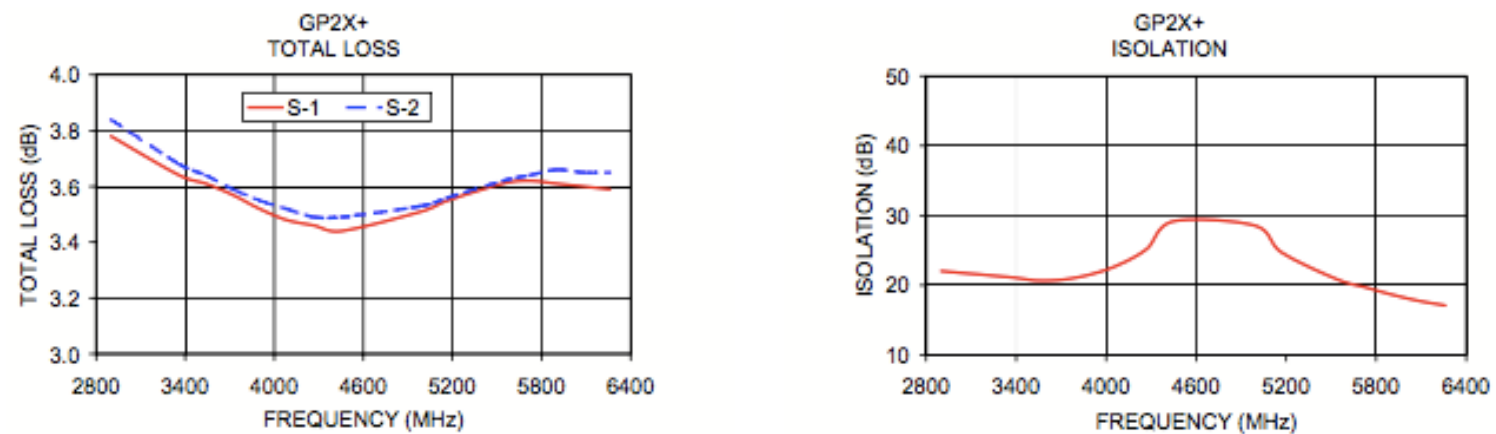

Figure 4.15: GP2X+ Typical Datasheet Total Loss and Isolation [46]

\subsubsection{Coupler}

Mini-Circuits SYBD-16-53HP+, a high power bi-directional coupler, is used couple the signal feeding the LO port of the mixer off to the transmit antenna. The SYBD-16-53HP+ operates from $2700-5000 \mathrm{MHz}$ with a $16 \mathrm{~dB}$ coupling factor while providing $0.2 \mathrm{~dB}$ mainline loss and 20dB directivity [47]. The datasheet specifications are shown below in Table 4.6. Typical loss, coupling factor, and directivity versus frequency are shown in Figure 4.16. 
Table 4.6: SYBD-16-53HP+ Datasheet Specifications

\begin{tabular}{|c|c|c|c|c|c|c|c|c|}
\hline \multirow{2}{*}{$\begin{array}{c}\text { FREQ. } \\
\text { (MHz) } \\
\mathrm{f}_{\mathrm{L}}-\mathrm{f}_{\mathrm{U}}\end{array}$} & \multicolumn{2}{|c|}{$\begin{array}{l}\text { COUPLING } \\
\text { (dB) }\end{array}$} & \multicolumn{2}{|c|}{$\begin{array}{l}\text { MAINLINE LOSS } \\
\text { (dB) }\end{array}$} & \multicolumn{2}{|c|}{$\begin{array}{l}\text { DIRECTIVITY } \\
\text { (dB) }\end{array}$} & \multirow{2}{*}{$\begin{array}{c}\text { VSWR } \\
\text { (:1) } \\
\\
\text { Typ. }\end{array}$} & \multirow{2}{*}{$\begin{array}{l}\text { POWER } \\
\text { INPUT } \\
\text { (W) } \\
\text { Max. }\end{array}$} \\
\hline & Nom. & Flatness & Typ. & Max. & Typ. & Min. & & \\
\hline $2700-5000$ & & & 0.20 & 0.70 & 18 & 10 & 1.20 & \\
\hline $2700-3000$ & $18.1 \pm 0.8$ & \pm 0.5 & 0.12 & 0.40 & 20 & 13 & 1.15 & 25 \\
\hline $3000-3600$ & $17.4 \pm 0.8$ & \pm 0.7 & 0.15 & 0.45 & 18 & 13 & 1.15 & 25 \\
\hline $3600-4500$ & $16.3 \pm 0.7$ & \pm 0.7 & 0.20 & 0.50 & 16 & 11 & 1.25 & 20 \\
\hline $4500-5000$ & $15.7 \pm 0.7$ & \pm 0.3 & 0.24 & 0.70 & 15 & 10 & 1.30 & 20 \\
\hline
\end{tabular}

1. Mainline loss includes theoretical power loss at coupled port.
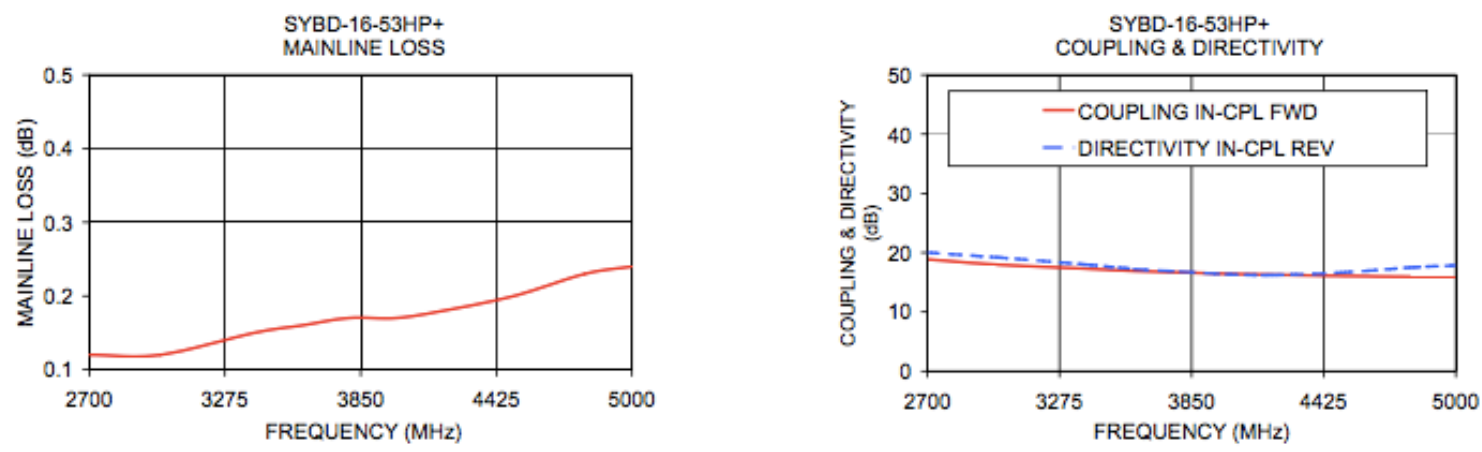

Figure 4.16: SYBD-16-53HP+ Typical Datasheet Loss Coupling and Directivity [47]

\subsubsection{Output Power Attenuator}

The transmit signal is attenuated further with a second resistive PI pad attenuator, as discussed in Section 4.4.3.3. Selecting $\mathrm{R}_{1}=\mathrm{R}_{3}=51 \Omega$ and $\mathrm{R}_{2}=3 \mathrm{k} \Omega$ meets the attenuator requirements with $41.56 \mathrm{~dB}$ attenuation and $50.15 \Omega$ input and output impedance.

\subsubsection{Receiver Components}

Each individual component in the receiver chain is discussed below. Typical datasheet performance is shown for critical RF parameters that impact system performance. Required external components and voltage rails are also discussed. For manually designed filters and attenuators that don't have datasheets, calculated or simulated performance is shown. 


\subsubsection{Band Pass Filter}

The first component in the receiver chain is a BPF, which is used to attenuate all signals not in the intended operating frequency band. This isolates the receiver components from high power out of band jammers, and prevents the LNA and other components from being saturated when hit with these unwanted signals. The BPF used in the receiver is the same component that is used in the transmitter chain, TDK UWB Band Pass Filter DEA453960BT-3002B1. See Section 4.4.3.5 for details.

\subsubsection{Low Noise Amplifier}

The noise figure and gain of the low noise amplifier drive the receiver system's sensitivity limit. The Avago MGA-71543 Low Noise Amplifier with Mitigated Bypass Switch is used to meet the derived system sensitivity requirements. It can operate from $0.1-6 \mathrm{GHz}$ off a $3 \mathrm{~V}$ rail with a gain of $16 \mathrm{~dB}$, noise figure of $0.8 \mathrm{~dB}$, and IP $3_{\text {in }}$ of $3 \mathrm{dBm}$ [48]. The MGA-71543 has a maximum tolerable input power of $+10 \mathrm{dBm}$. It requires external input and output matching, a bias choke, and decoupling capacitors on the supply rails. Typical noise figure, gain, IP $3_{\text {in }}$ and $\mathrm{P} 1 \mathrm{~dB}_{\text {out }}$ are shown below in Figure 4.17. 

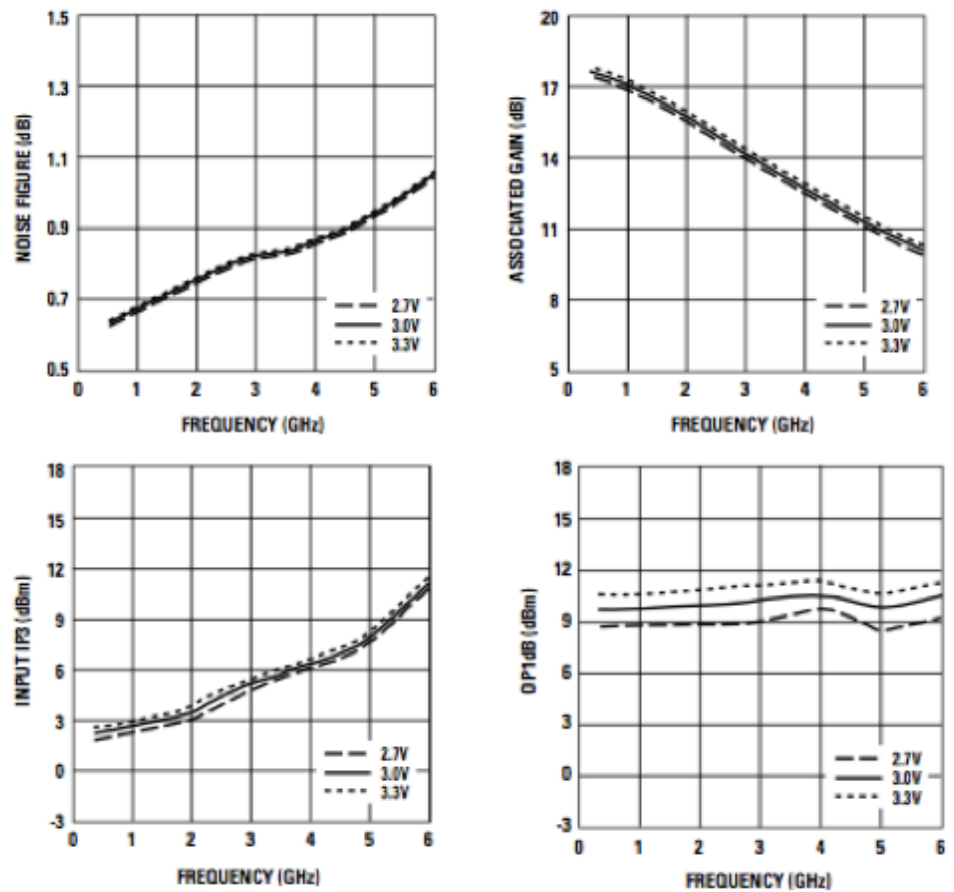

Figure 4.17: Typical Datasheet Performance [48]

\subsubsection{Rx Gain Block}

Analog Devices ADL5542 is a $50 \mathrm{MHz}$ to $6 \mathrm{GHz}$ RF/IF Gain Block. It is used after the LNA to further amplify the receive signal while providing a modest noise figure. Together they allow for the required system noise floor despite the high noise figure in the mixer and back end components. The ADL5542 has a fixed gain of $20 \mathrm{~dB}, I P 3_{\text {out }}$ of $40 \mathrm{dBm}, \mathrm{P} 1 \mathrm{~dB}$ out of $20.6 \mathrm{dBm}$, and noise figure of $3 \mathrm{~dB}$ at $900 \mathrm{MHz}$ [49]. It is capable of operating up to $6 \mathrm{GHz}$ off of a $5 \mathrm{~V}$ rail, and is internally matched to $50 \Omega$. It requires input and output $\mathrm{AC}$ coupling capacitors, power supply decoupling capacitors, and an external bias inductor. Typical datasheet performance for gain, noise figure, P1dB, and IP3 are shown below in Figure 4.18. 


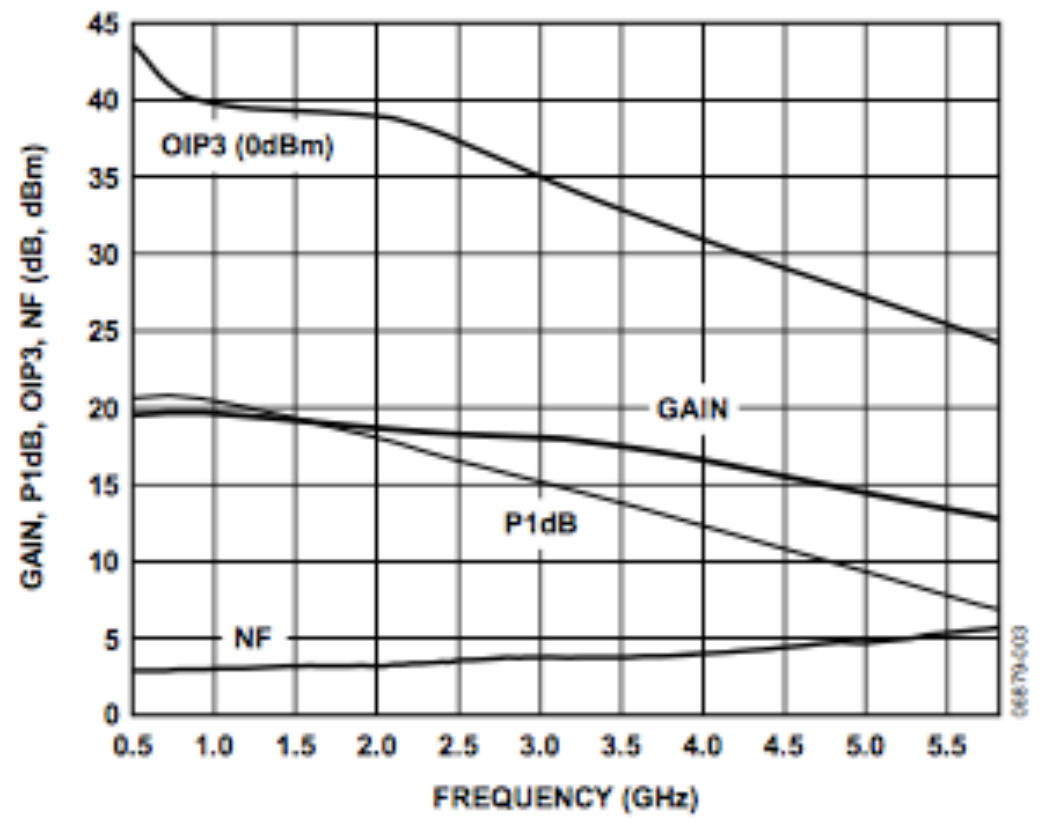

Figure 4.18: AD5542 Typical Datasheet Performance [49]

\subsubsection{Mixer}

The mixer chosen to combine the current transmit frequency with the received reflected frequencies is a Mini Circuits SIM-762H+ Frequency Mixer. It is a wide band mixer capable of operating from $2300-7600 \mathrm{MHz}$ with a required LO Power of $17 \mathrm{dBm}$. The IF bandwidth can range from $\mathrm{DC}$ to $3000 \mathrm{MHz}$ while maintaining a low typical conversion loss of $6 \mathrm{~dB}$. Table 4.7 below shows the datasheet specifications for conversion loss, isolation, and IP3 for various frequency ranges.

Table 4.7: SIM-762H+ Datasheet Specifications [50]

\section{Electrical Specifications}

\begin{tabular}{|c|c|c|c|c|c|c|c|c|c|}
\hline \multicolumn{2}{|c|}{$\begin{array}{l}\text { FREQUENCY } \\
(\mathrm{MHz})\end{array}$} & \multicolumn{3}{|c|}{$\begin{array}{l}\text { CONVERSION LOSS* } \\
(\mathrm{dB})\end{array}$} & \multicolumn{2}{|c|}{$\begin{array}{l}\text { LO-RF ISOLATION } \\
\text { (dB) }\end{array}$} & \multicolumn{2}{|c|}{$\begin{array}{l}\text { LO-IF ISOLATION } \\
\text { (dB) }\end{array}$} & \multirow{2}{*}{$\begin{array}{c}\begin{array}{c}\text { IP3 } \\
\text { (dBm) }\end{array} \\
\text { Typ. }\end{array}$} \\
\hline $\begin{array}{c}\mathrm{LO} / \mathrm{RI} \\
\mathrm{f}_{\mathrm{L}}-\mathrm{f}_{\mathrm{u}}\end{array}$ & IF & Typ. & $\sigma$ & Max. & Typ. & Min. & Typ. & Min. & \\
\hline $2300-7600$ & DC-3000 & & & & & & & & \\
\hline $2300-3200$ & & 6.0 & 0.1 & 9.0 & 35 & 27 & 21 & 13 & 25 \\
\hline $3200-3700$ & & 5.8 & 0.1 & 7.0 & 31 & 26 & 23 & 18 & 25 \\
\hline $3700-4200$ & & 5.9 & 0.2 & 7.4 & 32 & 26 & 25 & 19 & 25 \\
\hline $4200-7600$ & & 6.0 & 0.2 & 8.9 & 23 & 17 & 18 & 11 & 25 \\
\hline
\end{tabular}

$1 \mathrm{~dB}$ Compression: $+14 \mathrm{dBm}$ typ. 


\subsubsection{Discrete RC Low Pass Filter}

To attenuate the mixer harmonics, a single stage RC LPF with cutoff frequency of $1 \mathrm{GHz}$ is used. It also provides a $50 \Omega$ load to the mixer at the operating frequencies. The cutoff frequency is given by Eqn 4.6.

$$
f_{c}=\frac{1}{2 \pi R C}
$$

Eqn 4.6

Using $\mathrm{R}=50 \Omega$ and $\mathrm{f}_{\mathrm{c}}=1 \mathrm{GHz}$, gives $\mathrm{C}=3.18 \mathrm{pF}$. Using this calculation as a starting point, real models of Murata capacitors [51] are imported to ADS and used to simulate the filter accounting for high frequency fly back due to non-ideal components and their parasitic inductance. After optimizing the design using ADS simulations, the final filter design uses multiple shunt capacitors cascaded together. The ADS schematic and simulation results are shown below in Figure 4.19 and Figure 4.20.

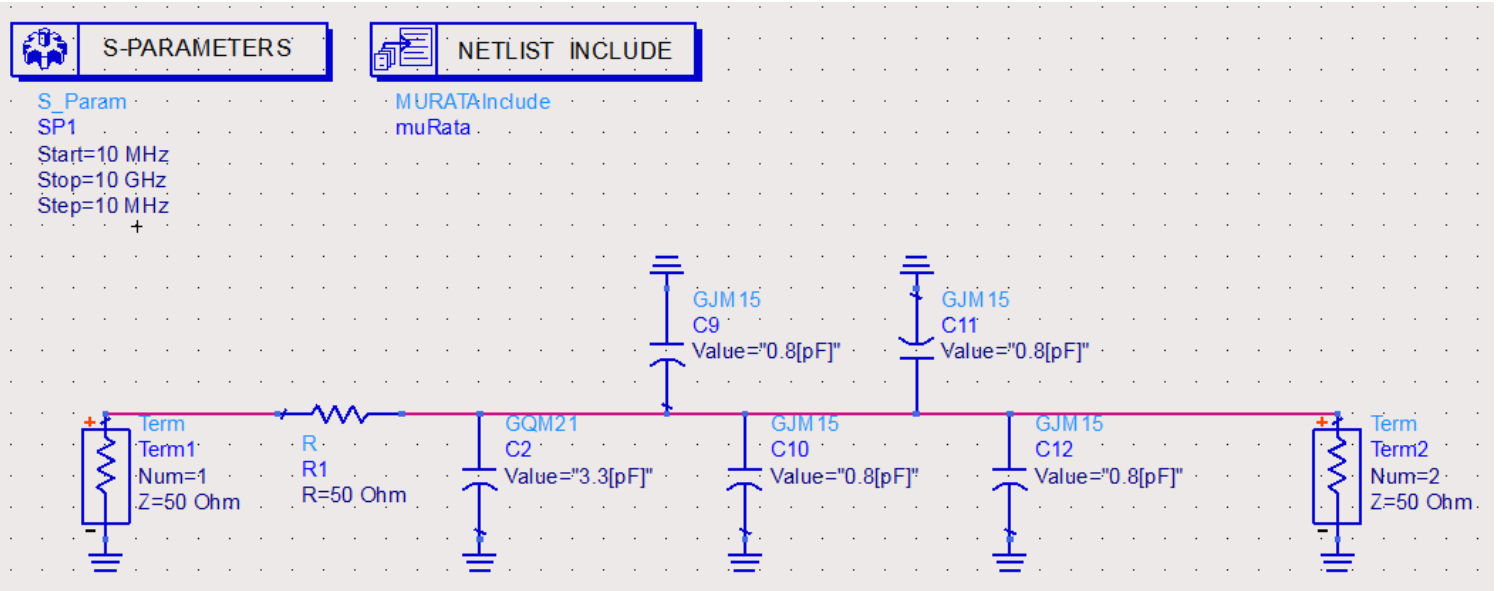

Figure 4.19: RC LPF ADS Schematic 

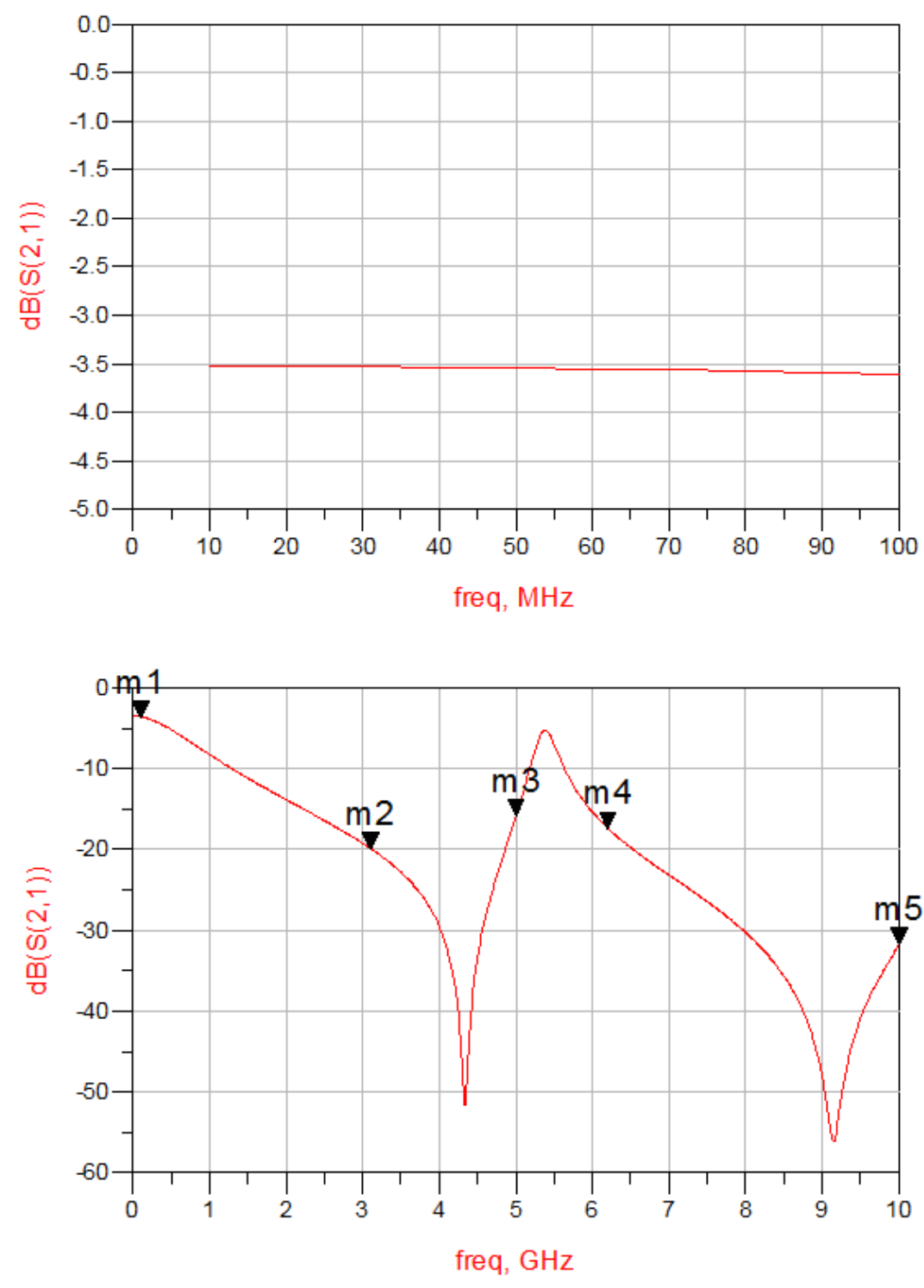

$\mathrm{m} 1$ freq $=100.0 \mathrm{MHz}$ $\mathrm{dB}(\mathrm{S}(2,1))=-3.602$ $\mathrm{m} 2$ freq $=3.100 \mathrm{GHz}$ $\mathrm{dB}(\mathrm{S}(2,1))=-19.857$ m3 freq $=5.000 \mathrm{GHz}$ $\mathrm{dB}(\mathrm{S}(2,1))=-15.804$ $\mathrm{m} 4$ freq $=6.200 \mathrm{GHz}$ $\mathrm{dB}(\mathrm{S}(2,1))=-17.382$ $\mathrm{m} 5$ freq $=10.00 \mathrm{GHz}$ $\mathrm{dB}(\mathrm{S}(2,1))=-31.716$

Figure 4.20: RC LPF ADS Simulation Results

This filter has $3.5 \mathrm{~dB}$ insertion loss in the operating band, $633 \mathrm{kHz}-6.33 \mathrm{MHz}$. It's out of band attenuation from $3.1-5 \mathrm{GHz}$ is better than $15 \mathrm{~dB}$, and better than $17 \mathrm{~dB}$ from $6.2-10 \mathrm{GHz}$.

\subsubsection{Active Low Pass Filter}

The active low pass filter limits the IF frequencies on the mixer output and sets the noise bandwidth of the receiver. The design is a Multiple Feedback topology, second order, $1 \mathrm{~dB}$ ripple, 
Chebyshev low pass filter. A cutoff frequency of $6.825 \mathrm{MHz}$ is chosen, with $20 \mathrm{~dB}$ gain. Figure 4.21 and Figure 4.22 below show the filter schematic and simulated gain and attenuation versus frequency.

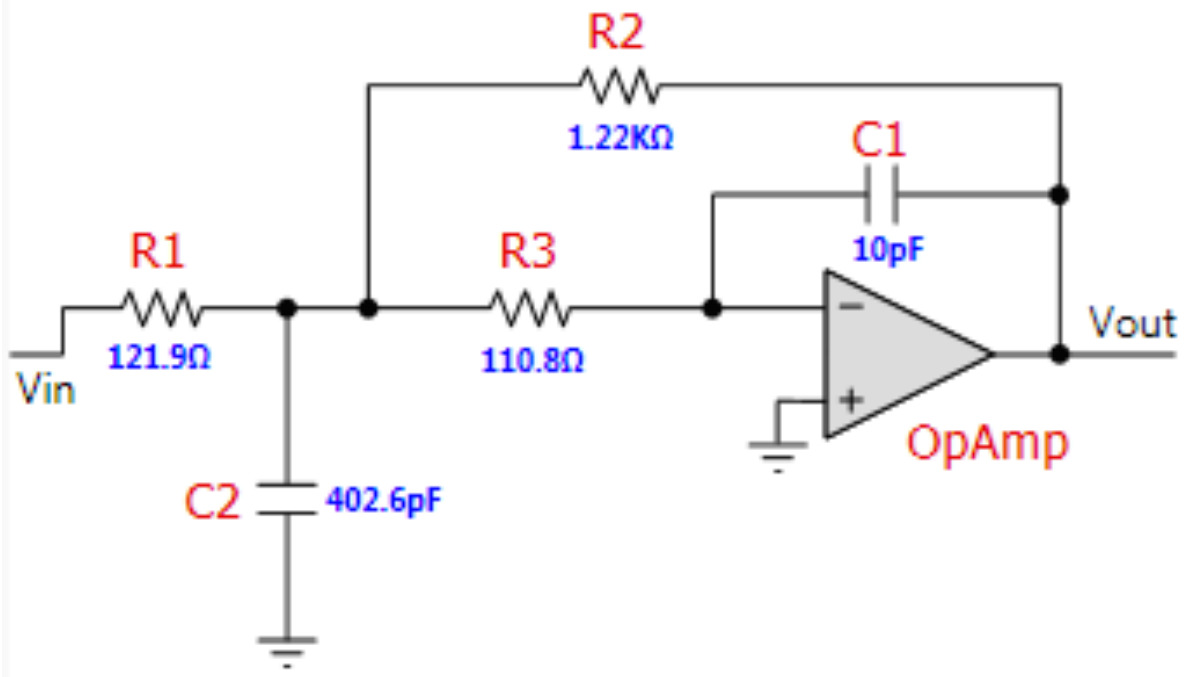

Figure 4.21: Active LPF Schematic

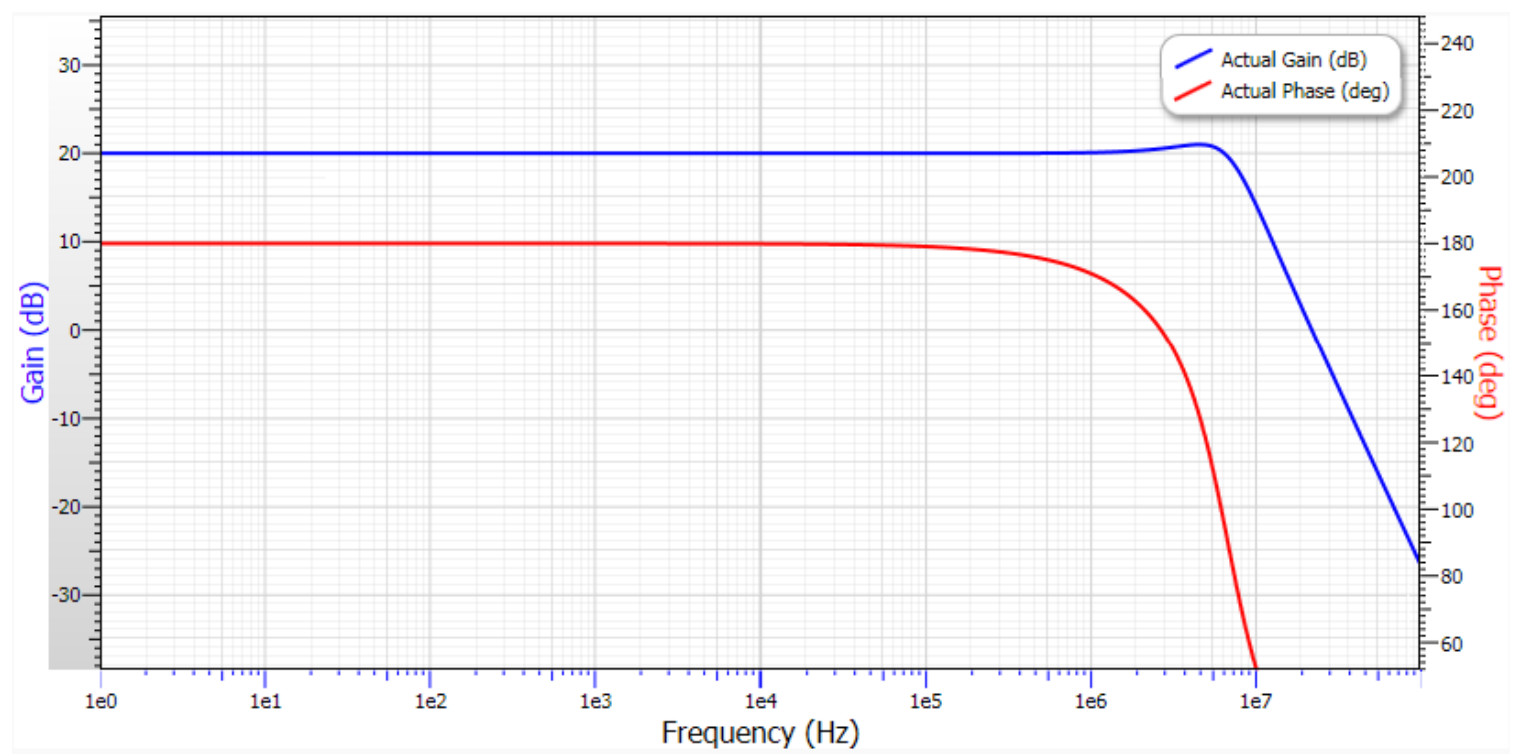

Figure 4.22: Active LPF Simulation Results

Standard component values close to the above schematic values are selected, giving the final filter component values below in Table 4.8. 
Table 4.8: Active LPF Schematic Values

\begin{tabular}{lll}
\hline Component & Ideal Value & Final Value \\
\hline $\mathbf{R}_{\mathbf{1}}$ & $121.9 \Omega$ & $124 \Omega$ \\
$\mathbf{R}_{\mathbf{2}}$ & $1220 \Omega$ & $1240 \Omega$ \\
$\mathbf{R}_{\mathbf{3}}$ & $110.8 \Omega$ & $113 \Omega$ \\
$\mathbf{C}_{\mathbf{1}}$ & $10 \mathrm{pF}$ & $10 \mathrm{pF}$ \\
$\mathbf{C}_{\mathbf{2}}$ & $402.6 \mathrm{pF}$ & $390 \mathrm{pF}$ \\
\hline
\end{tabular}

The Op Amp used in the amplifier is a Texas Instruments LMH6639 190MHz Rail-toRail Output Amplifier. It operates on a $\pm 5 \mathrm{~V}$ rail and has a $-3 \mathrm{~dB}$ bandwidth of $190 \mathrm{MHz}$, settling time of $33 \mathrm{~ns}$, slew rate of $172 \mu \mathrm{V} / \mathrm{s}$, and total harmonic distortion of $-60 \mathrm{dBc}$ [52]. Typical datasheet performance is shown below in Table 4.9.

Table 4.9: LMH6639 Typical Datasheet Performance [52]

\section{$\pm 5 V$ Electrical Characteristics}

Unless otherwise specified, all limits ensured for at $T_{J}=25^{\circ} \mathrm{C}, V_{S U P P L}= \pm 5 V, V_{O}=V_{C M}=G N D$, and $R_{L}=2 k \Omega$ to $V^{+} / 2$. Boldface limits apply at the temperature extremes.

\begin{tabular}{|c|c|c|c|c|c|c|c|}
\hline Symbol & Parameter & \multicolumn{2}{|c|}{ Conditions } & $\operatorname{Min}(1)$ & Typ $^{(2)}$ & $\operatorname{Max}^{(1)}$ & Units \\
\hline \multirow[t]{2}{*}{ BW } & \multirow[t]{2}{*}{$-3 d B$ BW } & \multicolumn{2}{|l|}{$A_{V}=+1$} & 150 & 228 & & \multirow{2}{*}{$\mathrm{MHz}$} \\
\hline & & \multicolumn{2}{|l|}{$A_{V}=-1$} & & 65 & & \\
\hline $\mathrm{BW}_{0.1 \mathrm{~dB}}$ & $0.1 \mathrm{~dB}$ Gain Flatness & \multicolumn{2}{|c|}{$\mathrm{R}_{\mathrm{F}}=2.26 \mathrm{k} \Omega, \mathrm{R}_{\mathrm{L}}=1 \mathrm{k} \Omega$} & & 18 & & $\mathrm{MHz}$ \\
\hline FPBW & Full Power Bandwidth & \multicolumn{2}{|c|}{$A_{V}=+1, V_{O U T}=2 V_{P p},-1 d B$} & & 29 & & $\mathrm{MHz}$ \\
\hline GBW & Gain Bandwidth Product & \multicolumn{2}{|l|}{$A_{V}=+1$} & & 90 & & $\mathrm{MHz}$ \\
\hline \multirow[t]{2}{*}{$\mathrm{e}_{\mathrm{n}}$} & \multirow[t]{2}{*}{ Input-Referred Voltage Noise } & \multirow[t]{2}{*}{$\mathrm{R}_{\mathrm{F}}=33 \mathrm{k} \Omega$} & $f=10 \mathrm{kHz}$ & & 19 & & \multirow{2}{*}{$\mathrm{nV} / \sqrt{\mathrm{Hz}}$} \\
\hline & & & $f=1 \mathrm{MHz}$ & & 16 & & \\
\hline \multirow[t]{2}{*}{$\mathrm{i}_{n}$} & \multirow[t]{2}{*}{ Input-Referred Current Noise } & \multirow[t]{2}{*}{$\mathrm{R}_{\mathrm{F}}=1 \mathrm{M} \Omega$} & $f=10 \mathrm{kHz}$ & & 1.13 & & \multirow{2}{*}{$\mathrm{pA} / \sqrt{\mathrm{Hz}}$} \\
\hline & & & $f=1 M H z$ & & 0.34 & & \\
\hline THD & Total Harmonic Distortion & \multicolumn{2}{|c|}{$\begin{array}{l}f=5 M H z, V_{O}=2 V_{P P}, A_{V}=+2 \\
R_{L}=1 \mathrm{k} \Omega\end{array}$} & & -71.2 & & $d B c$ \\
\hline DG & Differential Gain & \multicolumn{2}{|c|}{$\begin{array}{l}\text { NTSC, } A_{V}=+2 \\
R_{L}=150 \Omega\end{array}$} & & 0.11 & & $\%$ \\
\hline DP & Differential Phase & \multicolumn{2}{|c|}{$\begin{array}{l}\text { NTSC, } A_{V}=+2 \\
R_{L}=150 \Omega\end{array}$} & & 0.053 & & deg \\
\hline $\mathrm{T}_{\mathrm{S}}$ & Settling Time & \multicolumn{2}{|c|}{$V_{O}=2 V_{P \rho}, \pm 0.1 \%$} & & 33 & & ns \\
\hline SR & Slew Rate & \multicolumn{2}{|l|}{$A_{V}=-1^{(3)}$} & 140 & 200 & & $\mathrm{~V} / \mu \mathrm{s}$ \\
\hline$v_{\text {os }}$ & Input Offset Voltage & & & & 1.03 & $\begin{array}{l}5 \\
7\end{array}$ & $\mathrm{mV}$ \\
\hline $\mathrm{TC} \mathrm{V}_{\mathrm{OS}}$ & Input Offset Voltage Drift & $\operatorname{See}^{(4)}$ & & & 8 & & $\mu \mathrm{V} /{ }^{\circ} \mathrm{C}$ \\
\hline $\mathrm{l}_{8}$ & Input Bias Current & $\operatorname{See}^{(5)}$ & & & -1.40 & $\begin{array}{l}-2.6 \\
-3.25\end{array}$ & $\mu \mathrm{A}$ \\
\hline
\end{tabular}




\subsubsection{Variable Gain Amplifier}

To meet the large dynamic range requirements of the system, an Analog Devices AD603 Low Noise, $90 \mathrm{MHz}$ Variable Gain Amplifier is used at the system output. It allows the gain to be changed dynamically in accordance with the received signal power to provide the expected system output levels. It has pin-programmable gain ranges, and is configured to give adjustable gain from $9 \mathrm{~dB}$ to $51 \mathrm{~dB}$ with $9 \mathrm{MHz} \mathrm{BW}$ off of a $\pm 5 \mathrm{~V}$ supply. It has linear-in-dB gain control, with $\pm 0.5 \mathrm{~dB}$ typical gain accuracy. The gain depends on the tuning voltage, $\mathrm{V}_{\mathrm{G}}$, and is given by Eqn 4.7 below, where $\mathrm{V}_{\mathrm{G}}$ is between $-0.5 \mathrm{~V}$ and $+0.5 \mathrm{~V}$.

$$
G=40 V_{G}+30
$$

Eqn 4.7 [53]

Table 4.10 below shows relevant datasheet specifications. The required gain range to achieve the target output voltage of $3.3 \mathrm{Vpp}$ to $0.2 \mathrm{Vpp}$ for the ADC is $10.05 \mathrm{~dB}$ to $39.90 \mathrm{~dB}$. 
Table 4.10: AD603 Datasheet Specifications [53]

\section{SPECIFICATIONS}

\begin{tabular}{|c|c|c|c|c|c|}
\hline Parameter & Conditions & Min & Typ & Max & Unit \\
\hline $\begin{array}{l}\text { INPUT CHARACTERISTICS } \\
\text { Input Resistance } \\
\text { Input Capacitance } \\
\text { Input Noise Spectral Density' } \\
\text { Noise Figure } \\
1 \text { dB Compression Point } \\
\text { Peak Input Voltage } \\
\end{array}$ & $\begin{array}{l}\text { Pin } 3 \text { to } P \text { in } 4 \\
\text { Input short-circuited } \\
f=10 \mathrm{MHz} \text {, gain = maximum, } R_{5}=10 \Omega \\
f=10 \mathrm{MHz} \text {, gain = maximum, } R_{5}=10 \Omega\end{array}$ & 97 & $\begin{array}{l}100 \\
2 \\
1.3 \\
8.8 \\
-11 \\
\pm 1.4 \\
\end{array}$ & 103 & $\begin{array}{l}\Omega \\
\mathrm{pF} \\
\mathrm{nV} / \sqrt{\mathrm{Hz}} \\
\mathrm{dB} \\
\mathrm{dBm} \\
\mathrm{V}\end{array}$ \\
\hline $\begin{array}{l}\text { OUTPUT CHARACTERISTICS } \\
-3 \text { dB Bandwidth } \\
\text { Slew Rate } \\
\text { Peak Output }{ }^{2} \\
\text { Output Impedance } \\
\text { Output Short-Circuit Current } \\
\text { Group Delay Change vs. Gain } \\
\text { Group Delay Change vs. Frequency } \\
\text { Differential Gain } \\
\text { Differential Phase } \\
\text { Total Harmonic Distortion } \\
\text { Third-Order Intercept }\end{array}$ & $\begin{array}{l}V_{\text {OUT }}=100 \mathrm{mV} r \mathrm{~ms} \\
R_{L} \geq 500 \Omega \\
R_{L} \geq 500 \Omega \\
f \leq 10 \mathrm{MHz} \\
f=3 \mathrm{MHz} \text {; full gain range } \\
V_{G}=0 \mathrm{~V} ; \mathrm{f}=1 \mathrm{MHz} \text { to } 10 \mathrm{MHz} \\
f=10 \mathrm{MHz}, V_{\text {out }}=1 \mathrm{~V} \text { rms } \\
f=40 \mathrm{MHz} \text {, gain }=\text { maximum, } R_{5}=50 \Omega\end{array}$ & \pm 2.5 & $\begin{array}{l}90 \\
275 \\
\pm 3.0 \\
2 \\
50 \\
\pm 2 \\
\pm 2 \\
0.2 \\
0.2 \\
-60 \\
15\end{array}$ & & $\begin{array}{l}\mathrm{MHz} \\
\mathrm{V} / \mu \mathrm{s} \\
\mathrm{V} \\
\Omega \\
\mathrm{mA} \\
\mathrm{ns} \\
\mathrm{ns} \\
\% \\
\text { Degree } \\
\mathrm{dBc} \\
\mathrm{dBm}\end{array}$ \\
\hline $\begin{array}{l}\text { ACCURACY } \\
\text { Gain Accuracy, } f=100 \mathrm{kHz} \text {; Gain }(\mathrm{dB})=\left(40 \mathrm{~V}_{G}+10\right) \mathrm{dB} \\
\mathrm{T}_{\text {MiN }} \text { to } \mathrm{T}_{\text {MAX }} \\
\text { Gain, } \mathrm{f}=10.7 \mathrm{MHz} \\
\text { Output Offset Voltage } \\
\mathrm{T}_{\text {MiN }} \text { to } \mathrm{T}_{\text {Max }} \\
\text { Output Offset Variation vs. } \mathrm{V}_{G} \\
\mathrm{~T}_{\text {MIN }} \text { to } \mathrm{T}_{\operatorname{MAX}}\end{array}$ & $\begin{array}{l}-500 \mathrm{mV} \leq \mathrm{V}_{\mathrm{G}} \leq+500 \mathrm{mV} \\
\mathrm{V}_{\mathrm{G}}=-0.5 \mathrm{~V} \\
\mathrm{~V}_{\mathrm{G}}=0.0 \mathrm{~V} \\
\mathrm{~V}_{\mathrm{G}}=0.5 \mathrm{~V} \\
\mathrm{~V}_{\mathrm{G}}=0 \mathrm{~V} \\
-500 \mathrm{mV} \leq \mathrm{V}_{\mathrm{G}} \leq+500 \mathrm{mV}\end{array}$ & $\begin{array}{l}-1 \\
-1.5 \\
-10.3 \\
+9.5 \\
+29.3\end{array}$ & $\begin{array}{l} \pm 0.5 \\
-9.0 \\
+10.5 \\
+30.3\end{array}$ & $\begin{array}{l}+1 \\
+1.5 \\
-8.0 \\
+11.5 \\
+31.3 \\
20 \\
30 \\
20 \\
30\end{array}$ & $\begin{array}{l}\mathrm{dB} \\
\mathrm{dB} \\
\mathrm{dB} \\
\mathrm{dB} \\
\mathrm{dB} \\
\mathrm{mV} \\
\mathrm{mV} \\
\mathrm{mV} \\
\mathrm{mV}\end{array}$ \\
\hline $\begin{array}{l}\text { GAIN CONTROL INTERFACE } \\
\text { Gain Scaling Factor } \\
\mathrm{T}_{\text {MIN }} \text { to } \mathrm{T}_{\text {Max }} \\
\text { GNEG, GPOS Voltage Range } \\
\text { Input Bias Current } \\
\text { Input Offset Current } \\
\text { Differential Input Resistance } \\
\text { Response Rate } \\
\end{array}$ & $\begin{array}{l}100 \mathrm{kHz} \\
10.7 \mathrm{MHz} \\
\text { Pin } 1 \text { to Pin } 2 \\
\text { Full } 40 \mathrm{~dB} \text { gain change }\end{array}$ & $\begin{array}{l}39.4 \\
38 \\
38.7 \\
-1.2 \\
50\end{array}$ & $\begin{array}{l}40 \\
39.3 \\
100 \\
10 \\
50 \\
80 \\
\end{array}$ & $\begin{array}{l}40.6 \\
42 \\
39.9 \\
+2.0 \\
250\end{array}$ & $\begin{array}{l}\mathrm{dB} / \mathrm{V} \\
\mathrm{dB} / \mathrm{V} \\
\mathrm{dB} / \mathrm{V} \\
\mathrm{V} \\
\mathrm{nA} \\
\mathrm{nA} \\
\mathrm{M} \Omega \\
\mathrm{dB} / \mu \mathrm{s}\end{array}$ \\
\hline $\begin{array}{l}\text { POWER SUPPLY } \\
\text { Specified Operating Range } \\
\text { Quiescent Current } \\
\mathrm{T}_{\text {Miv }} \text { to } \mathrm{T}_{\operatorname{Max}}\end{array}$ & & \pm 4.75 & 12.5 & $\begin{array}{l} \pm 6.3 \\
17 \\
20\end{array}$ & $\begin{array}{l}\mathrm{v} \\
\mathrm{mA} \\
\mathrm{mA}\end{array}$ \\
\hline
\end{tabular}

\subsubsection{Component DC Voltage Supplies}

To supply the various voltage rails required by all the components, the system will receive $\pm 5 \mathrm{~V}$, and $+20 \mathrm{~V}$ from an external power supply. To generate the $+3 \mathrm{~V}$ and $+3.3 \mathrm{~V}$ rails required by other components, a Micrel MIC5335-3.3/3.0YMT Dual High Performance 300mA $\mu \mathrm{Cap}$ ULDO is used. This LDO takes an input voltage between $4.3 \mathrm{~V}$ and $5.5 \mathrm{~V}$, and outputs 
separate rails for both $3 \mathrm{~V}$ and $3.3 \mathrm{~V}$. The output rails have a $300 \mathrm{~mA}$ output each, and a $75 \mathrm{mV}$

dropout at that max current load. The datasheet specifications are shown below in Figure 4.23.

\section{Electrical Characteristics ${ }^{(5)}$}

$\mathrm{V}_{\mathrm{IN}}=\mathrm{EN} 1=\mathrm{EN} 2=\mathrm{V}_{\text {OUT }}+1.0 \mathrm{~V}$; higher of the two regulator outputs, $\mathrm{I}_{\text {OUTLDO1 }}=\mathrm{I}_{\text {OUTLDO2 }}=100 \mu \mathrm{A} ; \mathrm{C}_{\text {OUT1 }}=\mathrm{C}_{\text {OUT2 }}=1 \mu \mathrm{F} ;$ $T_{J}=25^{\circ} \mathrm{C}$, bold values indicate $-40^{\circ} \mathrm{C} \leq T_{J} \leq+125^{\circ} \mathrm{C}$, unless noted.

\begin{tabular}{|c|c|c|c|c|c|}
\hline Parameter & Conditions & Min & Typ & Max & Units \\
\hline \multirow[t]{2}{*}{ Output Voltage Accuracy } & Variation from nominal $V_{\text {OUT }}$ & -2.0 & & +2.0 & $\%$ \\
\hline & Variation from nominal $V_{\text {our }} ;-40^{\circ} \mathrm{C}$ to $+125^{\circ} \mathrm{C}$ & -3.0 & & +3.0 & $\%$ \\
\hline Line Regulation & $\mathrm{V}_{\mathrm{IN}}=\mathrm{V}_{\text {OUT }}+1 \mathrm{~V}$ to $5.5 \mathrm{~V} ;$ IouT $=100 \mu \mathrm{A}$ & & 0.02 & $\begin{array}{l}0.3 \\
0.6\end{array}$ & $\begin{array}{l}\% / V \\
\% / N\end{array}$ \\
\hline Load Regulation & $\mathrm{I}_{\text {OUT }}=100 \mu \mathrm{A}$ to $300 \mathrm{~mA}$ & & 0.3 & 2.0 & $\%$ \\
\hline Dropout Voltage (Note 6) & $\begin{array}{l}\text { loUT }_{\text {OUT }}=100 \mu \mathrm{A} \\
\text { loUT }=100 \mathrm{~mA} \\
\mathrm{I}_{\text {OUT }}=150 \mathrm{~mA} \\
\text { I OUT }_{\text {OU }}=300 \mathrm{~mA}\end{array}$ & & $\begin{array}{l}0.1 \\
25 \\
35 \\
75 \\
\end{array}$ & $\begin{array}{c}75 \\
100 \\
200 \\
\end{array}$ & $\begin{array}{l}\mathrm{mV} \\
\mathrm{mV} \\
\mathrm{mV} \\
\mathrm{mV}\end{array}$ \\
\hline Ground Current & $\begin{array}{l}\text { EN1 }=\text { High; EN2 }=\text { Low; lout }=100 \mu \text { A to } 300 \mathrm{~mA} \\
\text { EN1 }=\text { Low; EN2 }=\text { High; lout }=100 \mu \text { A to } 300 \mathrm{~mA} \\
\text { EN1 }=\text { EN2 }=\text { High; lout1 }=300 \mathrm{~mA}, \text { lout2 }=300 \mathrm{~mA}\end{array}$ & & $\begin{array}{c}90 \\
90 \\
150 \\
\end{array}$ & $\begin{array}{l}125 \\
125 \\
220 \\
\end{array}$ & $\begin{array}{l}\mu \mathrm{A} \\
\mu \mathrm{A} \\
\mu \mathrm{A}\end{array}$ \\
\hline Ground Current in Shutdown & $\mathrm{EN} 1=\mathrm{EN} 2=0 \mathrm{~V}$ & & 0.01 & 2 & $\mu \mathrm{A}$ \\
\hline Ripple Rejection & $\begin{array}{l}f=1 \mathrm{kHz} ; \text { Cout }=1.0 \mu \mathrm{F} \\
\mathrm{f}=20 \mathrm{kHz} ; \text { CoUT }=1.0 \mu \mathrm{F}\end{array}$ & & $\begin{array}{l}65 \\
45\end{array}$ & & $d B$ \\
\hline Current Limit & $\mathrm{V}_{\text {OUT }}=0 \mathrm{~V}$ & 340 & 550 & 950 & $\mathrm{~mA}$ \\
\hline Output Voltage Noise & $\mathrm{C}_{\text {OUT }}=1.0 \mu \mathrm{F} ; 10 \mathrm{~Hz}$ to $100 \mathrm{kHz}$ & & 90 & & $\mu \mathrm{V}_{\mathrm{RMS}}$ \\
\hline \multicolumn{6}{|l|}{ Enable Inputs (EN1 / EN2) } \\
\hline \multirow[t]{2}{*}{ Enable Input Voltage } & Logic Low & & & 0.2 & $\mathrm{~V}$ \\
\hline & Logic High & 1.1 & & & $\mathrm{~V}$ \\
\hline \multirow[t]{2}{*}{ Enable Input Current } & $\mathrm{V}_{\mathrm{IL}} \leq 0.2 \mathrm{~V}$ & & 0.01 & 1 & $\mu \mathrm{A}$ \\
\hline & $\mathrm{V}_{\mathrm{IH}} \geq 1.0 \mathrm{~V}$ & & 0.01 & 1 & $\mu \mathrm{A}$ \\
\hline \multicolumn{6}{|c|}{ Turn-on Time (See Timing Diagram) } \\
\hline Turn-on Time (LDO1 and 2) & $\mathrm{C}_{\mathrm{OUT}}=1.0 \mu \mathrm{F}$ & & 30 & 100 & $\mu \mathrm{s}$ \\
\hline
\end{tabular}

Figure 4.23: Micrel MIC5335 Datasheet Specifications [54]

\subsection{Final System Design}

\subsubsection{System Schematic}

Supporting components for each part are compiled from datasheets and app notes and used to create the initial system schematic, which can be seen in APPENDICES

APPENDIX A: System Schematic. The schematic is broken up into four sections. The FMCW Generator schematic page includes the FMCW generation from the function generator 
sawtooth input to the stepped impedance LPF after the frequency multiplier. It also contains the voltage regulator, which supplies nets for all of the components that run off of $3.0 \mathrm{~V}$ or $3.3 \mathrm{~V}$. The next schematic page is the transmitter, which includes the transmitter chain from the first gain block to the transmit antenna output and two coupled LO outputs. The two receiver schematics are identical, and include the entire receive chain from the antenna input to the VGA output. Zero ohm resistors are added to separate each component in order to facilitate individual component characterization and debug.

\subsubsection{Calculated System Performance}

The final system design is analyzed to ensure performance is met for all system requirements. Detailed level plans that calculate system performance throughout each component in the system chain are compiled in APPENDIX C: Transmitter Level Plan, and APPENDIX D: Receiver Level Plan. Typical component performance is referenced from their datasheets, and used to calculate system performance for key parameters. The transmitter level plan shows calculations for transmit power, LO power, and third harmonic levels. The receiver level plan shows calculations for cascaded noise figure, total system gain, maximum input signal drive, and cascaded third order intercept. Each of these system calculations are compared to the system specifications in Table 4.1 and Table 4.2 to ensure the system design will meet the required performance.

\subsubsection{Transmit Power}

Tx Spec 1 of Table 4.1 states that the output power needs to be less than $-41.3 \mathrm{dBm} / \mathrm{MHz}$ from 3.1-4.9GHz. The transmitter level plan calculates transmit power starting with the VCO typical output power, and adding component gain or loss. The calculated nominal output power is $-41.51 \mathrm{dBm} / \mathrm{MHz}$. The same transmit chain also drives the LO ports of the Rx mixers. The 
mixer requires between 14 and $20 \mathrm{dBm}$ at the LO port, and the level plan shows a calculated value of $16.45 \mathrm{dBm}$.

\subsubsection{Transmitter Harmonic Generation}

Because $2 * F_{\text {low }}>F_{\text {high }}$, all harmonics of the transmit band fall outside of the transmission bandwidth, so they can easily be attenuated with filtering. Tx Spec 2 from Table 4.1 requires the output power is below $-51.3 \mathrm{dBm} / \mathrm{MHz}$ above $10.6 \mathrm{GHz}$, which corresponds to the transmitter third harmonic. The level plan tracks the third harmonic level through each stage by taking the power of the harmonic from the previous stage and adding the stage gain, and summing that result with the harmonic level generated at the stage itself. The level plan shows a third harmonic output at the antenna of $-110.8 \mathrm{dBm} / \mathrm{MHz}$, which easily meets the system specification.

\subsubsection{Receiver Minimum Detectable Signal}

The first step in finding the minimum detectable signal of the receiver is to find the total cascaded noise figure. The cascaded noise figure of two components is given by Eqn 4.8 below, with all numbers in the linear domain.

$$
F_{T}=F_{1}+\frac{F_{2}-1}{G_{1}}
$$

Passive lossy components add directly to the noise figure in $\mathrm{dB}$. The receiver level plan shows a cascaded noise figure of $3.94 \mathrm{~dB}$ for the entire receiver chain.

The noise bandwidth set by the active LPF is $6.825 \mathrm{MHz}$, which gives a thermal noise floor of $-105.66 \mathrm{dBm}$ over that bandwidth. Using a required SNR of $6 \mathrm{~dB}$ to detect the signal, gives a receiver minimum detectable signal of $-95.70 \mathrm{dBm}$. Rx Spec 2 of Table 4.2 gives a required minimum detectable signal of $-85 \mathrm{dBm}$, which leaves $10 \mathrm{~dB}$ of margin. 


\subsubsection{Receiver Maximum Detectable Signal}

The maximum input signal according to $\mathrm{Rx}$ Spec 1 in Table 4.2 is required to be $-41.3 \mathrm{dBm} / \mathrm{MHz}$. The receiver level plan propagates a $-41.3 \mathrm{dBm}$ signal through each of the receiver components, and shows the input power to each component. Each component's $\mathrm{P}_{1 \mathrm{~dB}}$ is well above the maximum input power it will see; therefore, the system can handle the required maximum signal input power.

\subsubsection{Receiver Minimum and Maximum System Gain}

Rx Specs 3.1 and 3.2 in Table 4.2 shows the required receiver chain total cascaded gain must cover at least $55.65 \mathrm{~dB}$ to $75 \mathrm{~dB}$. The receiver level plan calculates the gain and loss of each component, including the maximum and minimum adjustable gain of the VGA. The system maximum and minimum gains works out to $89.8 \mathrm{~dB}$ and $47.8 \mathrm{~dB}$ respectively, which meets the system specifications.

\subsubsection{Receiver Linearity}

Receiver linearity is important because the receiver will be subjected to multiple input signals simultaneously. If the receiver has poor linearity, these desired input signals will mix with each other and create higher order products that can also fall in band. Figure 4.24 below depicts two desired fundamental input signals and the resulting second, third, and fifth order mixing products. 


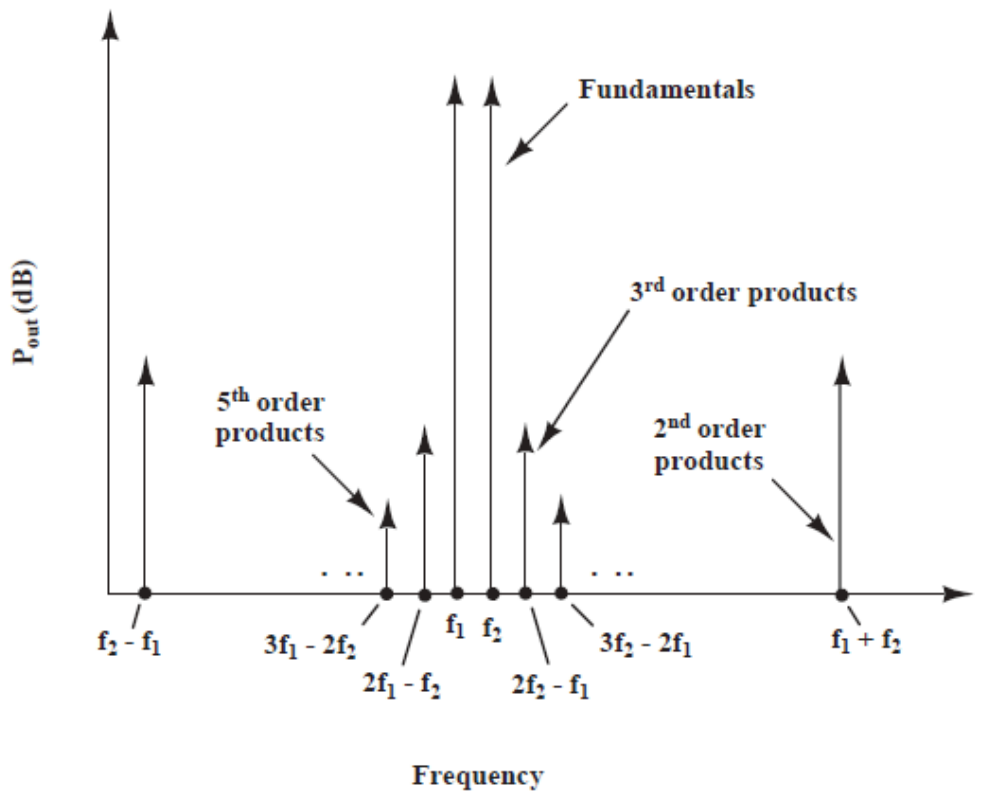

Figure 4.24: Higher Order Mixing Products [55]

The even mixing products fall far from the fundamental frequencies, but the odd mixing products are closely spaced, proportional to the frequency spacing between the two fundamental tones. These odd, higher order mixing products that fall in band will appear to the receiver as lower power reflections off of additional objects at different distances, thus these undesired in band products are designated as "ghost objects." To prevent the receiver from seeing ghost objects, the receiver must have high enough linearity to keep the mixing products below the system noise floor.

The IM3 product output level depends on the receiver IP3 as well as the power of the two input tones, and is given by Eqn 4.9 below.

$$
P_{I M D 3}(d B m)=2 * P_{1}+P_{2}-2 * I P 3(d B m)
$$

Eqn 4.9

To find the receiver IP3, the IP3 of the individual components is cascaded in the level plan. Eqn 4.10 below shows the IP3 cascaded formula for two components. 


$$
I P 3_{T}(m W)=\frac{1}{\frac{1}{I P 3_{1}}+\frac{G_{1}}{I P 3_{2}}}(m W)
$$

Eqn 4.10

Using this method, the level plan shows the total cascaded IP3 for the receiver chain of $-5.5 \mathrm{dBm}$. The worst-case scenario is two input signals at the maximum detectable signal level of $-41.3 \mathrm{dBm}$. Inputting these numbers to Eqn 4.9 gives a $\mathrm{P}_{\mathrm{IMD} 3}$ of $-112.9 \mathrm{dBm}$, which is below the system noise floor and therefore the receiver will not detect any ghost objects.

\subsection{PCB Layout}

Component datasheets were referenced to create a library of land pattern footprints for each part using DipTrace Pattern Editor. Each footprint was linked to its corresponding schematic symbol that had been previously created and added to a library in DipTrace Component Editor. Then the schematic from DipTrace Schematic Capture was ported into the DipTrace PCB Layout Editor. From here the PCB design started.

Due to budget constraints, the board was required to be two layer 0.062 " thick FR-4 with loz copper plating from Advanced Circuits PCBs. The available process used Isola FR402, which has a dielectric constant of 4.25 and loss tangent of 0.015 [56]. The material properties are used to calculate the required physical dimensions for the $50 \Omega$ RF microstrip traces. Figure 4.25 below shows the material parameters input to ADS LineCalc. The LineCalc results show that a 122 mil trace width will result in a $50.28 \Omega$ characteristic impedance trace, calculated at a frequency of $5 \mathrm{GHz}$. 


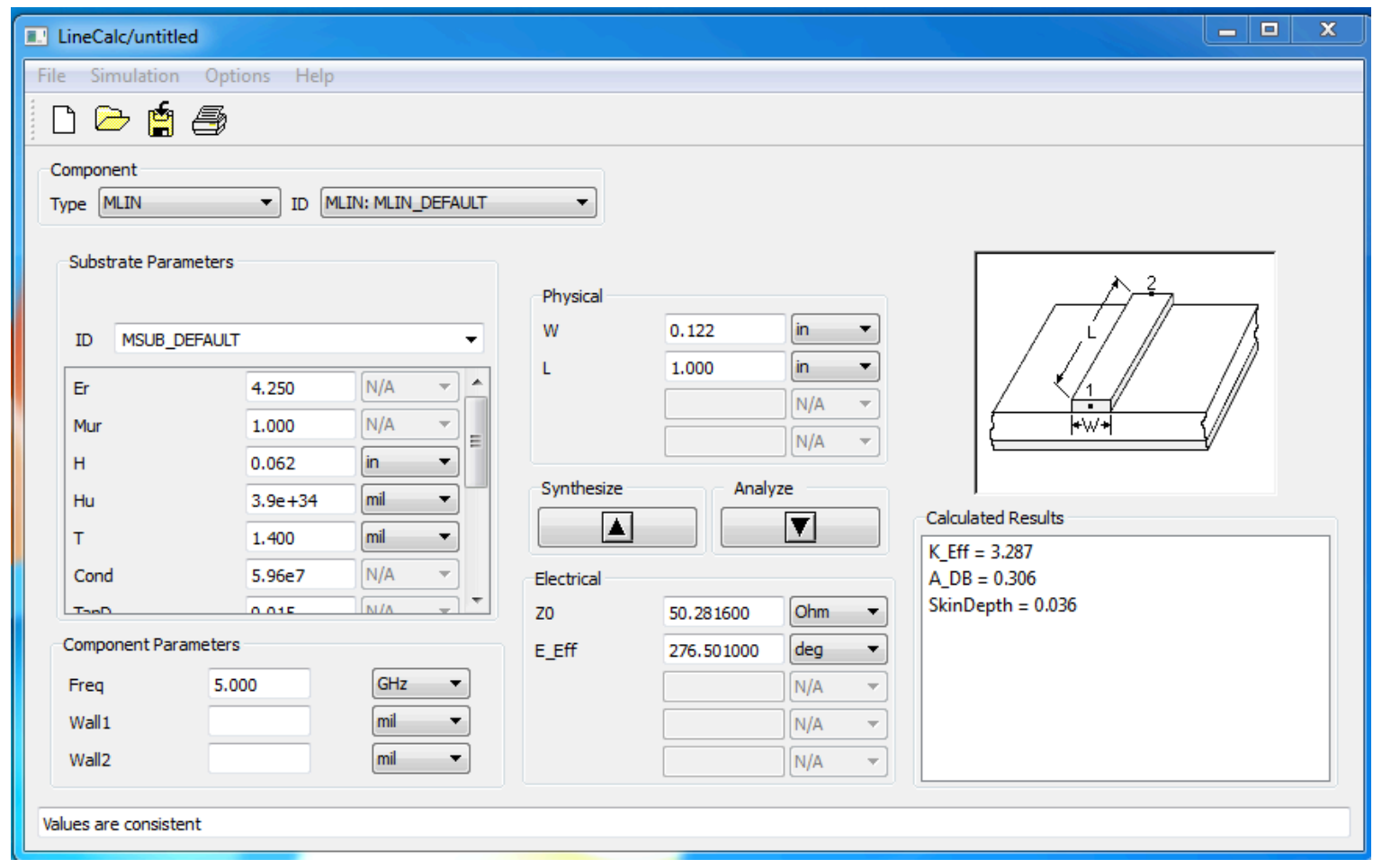

Figure 4.25: ADS LineCalc Output

$50 \Omega$ net properties are defined in DipTrace PCB Layout for 122 mil trace width with 244mil ground clearance on either side. The ground clearance sufficiently ensures the coupling to ground is dominated by the ground underneath the microstrip line rather than the ground on the same layer as the trace, thus making it a $50 \Omega$ characteristic impedance per the above calculation.

The two-layer board uses the top layer to route signals and the bottom layer as an uninterrupted reference ground. However, in the digital section, some signals via to the bottom layer for short distances to cross over other lines. A few supply voltage lines for RF components need to jump across the RF traces as well. Rather than disturbing the RF ground, pads were placed on the PCB to allow external wires to jump the DC voltage over the RF traces.

In the RF section, vias were stitched as often as possible to reduce inductance to ground and improve isolation between signal traces. The fabrication design rules allowed for 35 holes per square inch maximum. The layout was placed conservatively, with a lot of space between 
components and thus longer traces. While this increases insertion loss between components, which will reduce system sensitivity and output power, it was done to prevent coupling between various signal traces on the PCB. For instance, if the transmit signal coupled to the receive signal path, it would appear to the system as if there was an object at distance zero. The trace width of the $50 \Omega$ lines also requires large separation between the components. The $122 \mathrm{mil}$ traces, which are defined by the PCB dielectric constant and stack up height, are much wider than the components and their connecting pins. The delta between trace width and component connections will cause an impedance discontinuity which creates reflections, reducing return loss and incurring additional loss.

Zero ohm resistors were inserted between every component in the chain to facilitate characterization of the front-end performance. While this slightly decreases overall performance, it allows individual characterization of each component by removing the resistors and pig tailing directly into the input and output of the component.

In addition to the transmitter and receiver circuitry, a coupon trace was added to the PCB to characterize the $50 \Omega$ trace properties. The trace is one quarter wavelength at $4 \mathrm{GHz}$, and has placement for either a pigtail or SMD component on either side. It is used to measure trace insertion loss, return loss, and characteristic impedance.

The board component placement and layout can be seen below in Figure 4.26. The transmitter circuitry is in the center of the board with both receivers on the top and bottom. The RF input and outputs are on the left of the board, and the chain flows to the digital section on the right side. The voltage regulators are centered on the right edge of the board. Finally, the trace coupon can be seen in the lower right corner. 


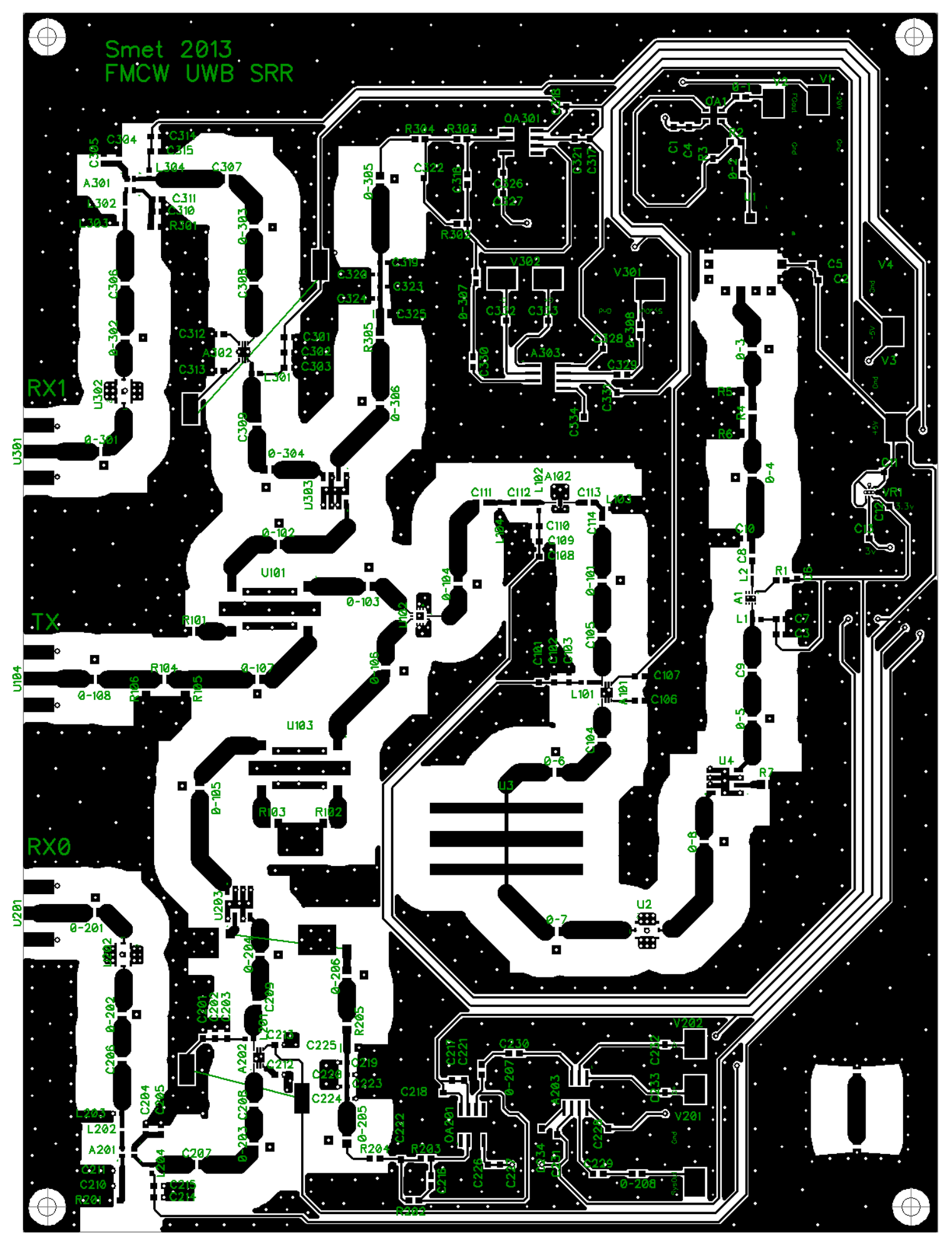

Figure 4.26: PCB Layout 


\section{Results}

After the fabricated PCB was received the components were mounted using a solder reflow oven. Each facet of the system was characterized to determine if it was performing as intended. Throughout the process various pieces of the design were modified to improve performance. Commonly this included RF input and output matching for components or adjustments in the DC bias network. The following sections discuss the performance of the PCB itself, as well as each of the individual components. Measured data for each component on the PCB is presented.

\subsection{PCB Assembly}

The 6" by 8" board complete with silkscreen and solder mask is pictured in Figure 5.1.

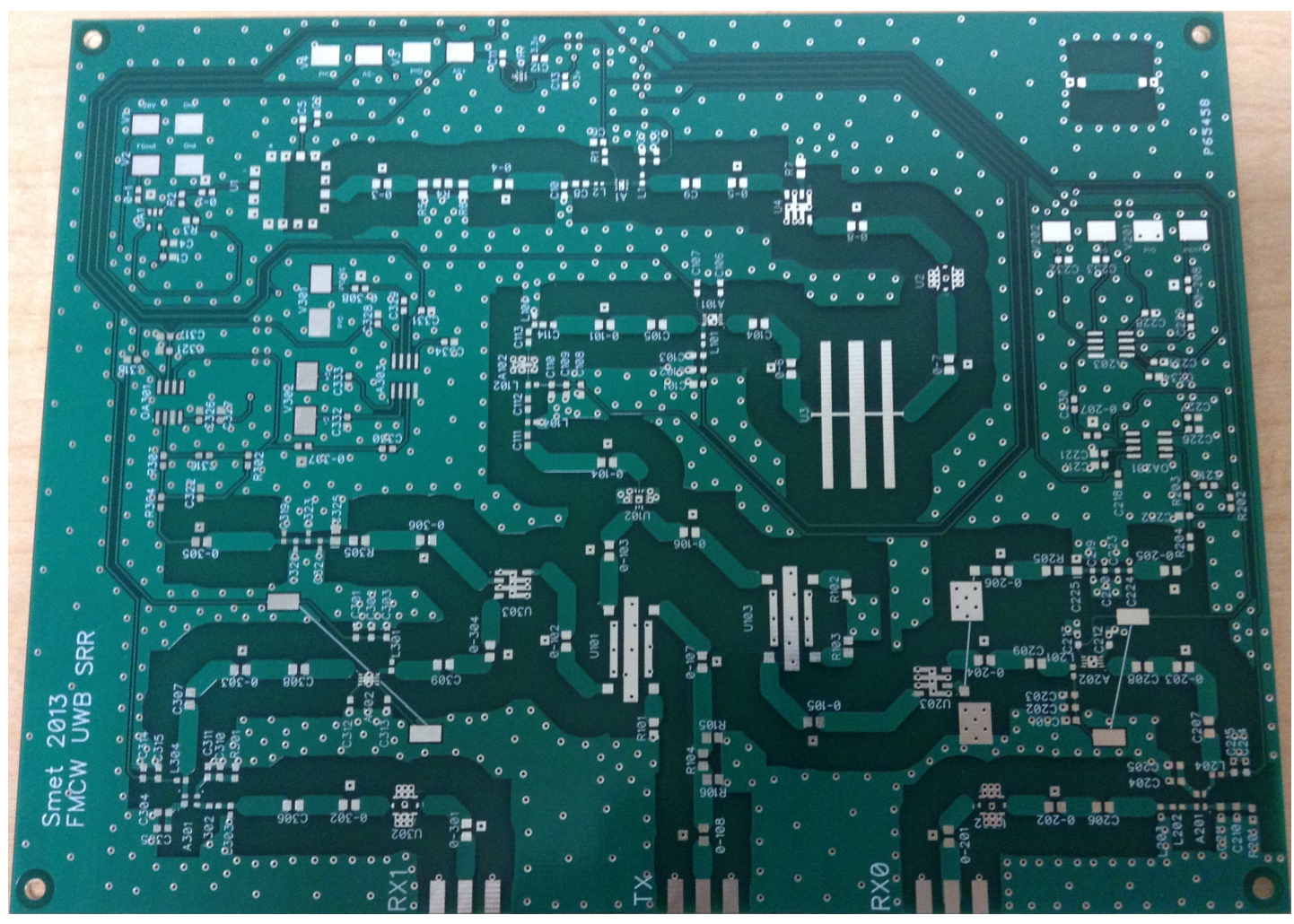

Figure 5.1: Bare PCB 
Solder paste was manually applied to each pad and the components were applied. The entire board went through a reflow oven to flow the solder and mount each component in its place. After inspection, a few components were manually remounted with a soldering iron and heat gun to ensure a proper connection. The assembled PCB is pictured in Figure 5.2.

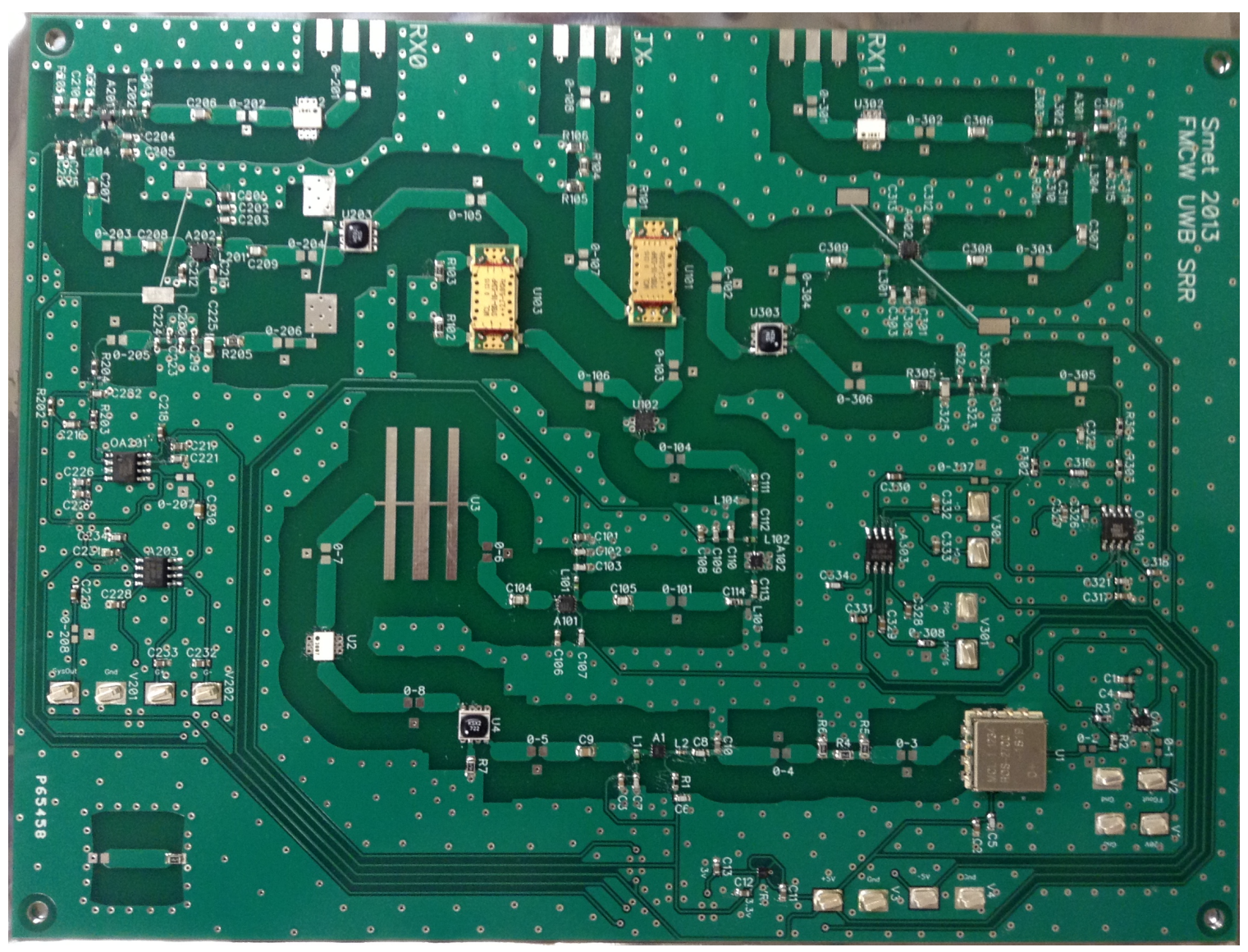

Figure 5.2: Assembled PCB

\subsection{PCB Characterization}

The first validation done on the board was to find the characteristics of the $50 \Omega \mathrm{RF}$ traces. The insertion loss and impedance of the RF coupon trace was measured with a VNA from $100 \mathrm{kHz}$ up to $6 \mathrm{GHz}$. Figure 5.3 below shows the insertion loss data for the RF coupon trace across frequency. 


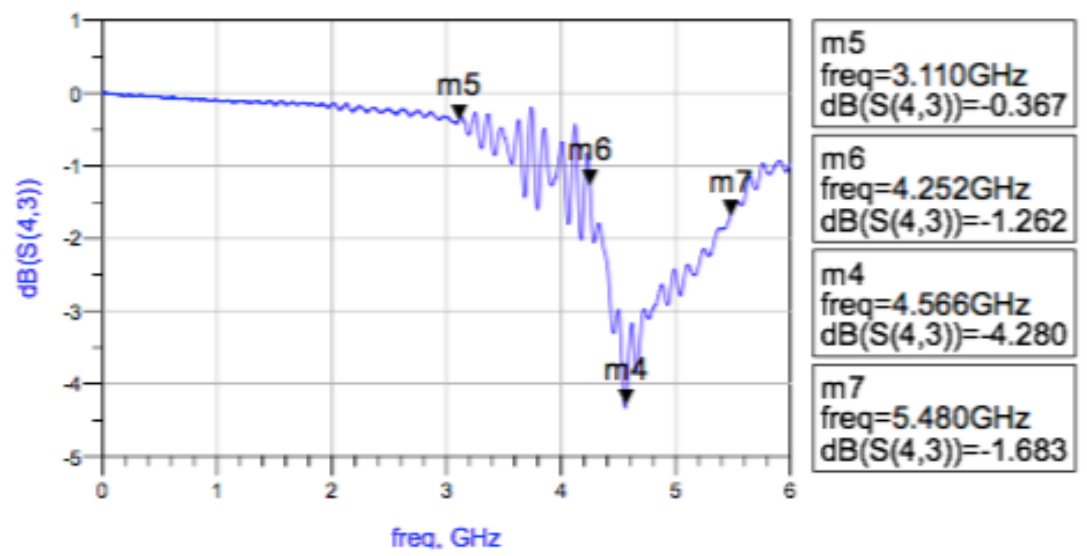

Figure 5.3: RF Coupon Insertion Loss

The insertion loss behaves as expected up to $3 \mathrm{GHz}$, increasing linearly with frequency.

At $3.11 \mathrm{GHz}$ the $421 \mathrm{mil}$ long trace has $0.367 \mathrm{~dB}$ of insertion loss. Above $3 \mathrm{GHz}$ the $\mathrm{S} 21$

measurement shows a large ripple in the response which peaks at $4.566 \mathrm{GHz}$ where it hits $4.28 \mathrm{~dB}$ of insertion loss.

Similarly, Figure 5.4 shows the return loss and impedance across frequency for each port of the coupon trace. 


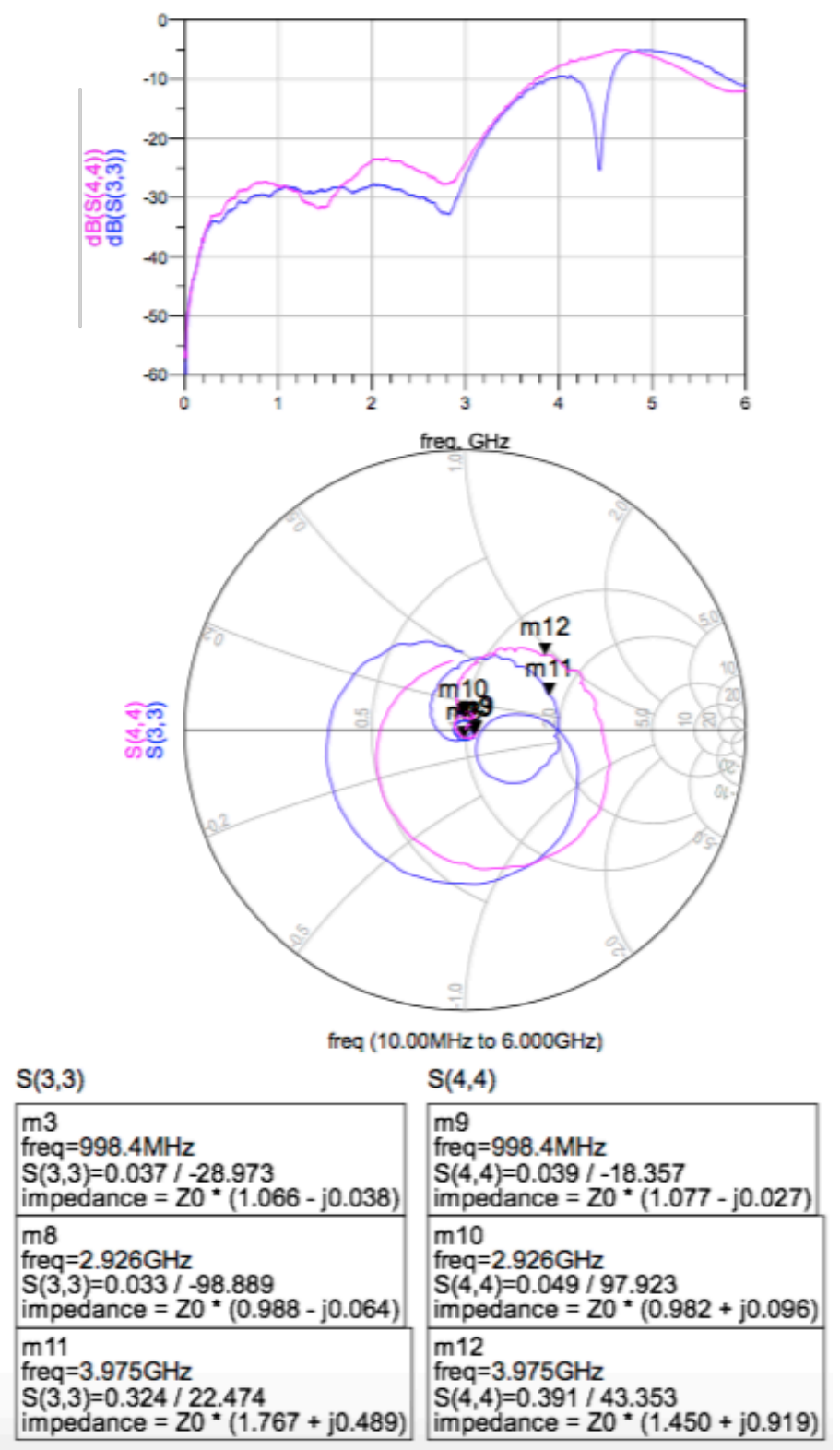

Figure 5.4: RF Coupon Return Loss and Impedance

The trace impedance is $50 \Omega$ as expected at lower frequencies as well. At $2.926 \mathrm{GHz}$ it has a VSWR of $1.104: 1$, and at $3.975 \mathrm{GHz}$ the VSWR is $2.284: 1$. The resonant response at high frequencies only gives the traces good performance below $3 \mathrm{GHz}$. As frequency increases so do the parasitic capacitances and inductances, and eventually they begin to dominate and change the 
trace response. The insertion loss and return loss data in Figure 5.3 and Figure 5.4 show that the RF traces do not have good performance all the way up to $5 \mathrm{GHz}$ where the system will operate.

Next, the electrical length of the RF coupon trace was measured by leaving one of the ends of the trace open, which is shown in Figure 5.5.
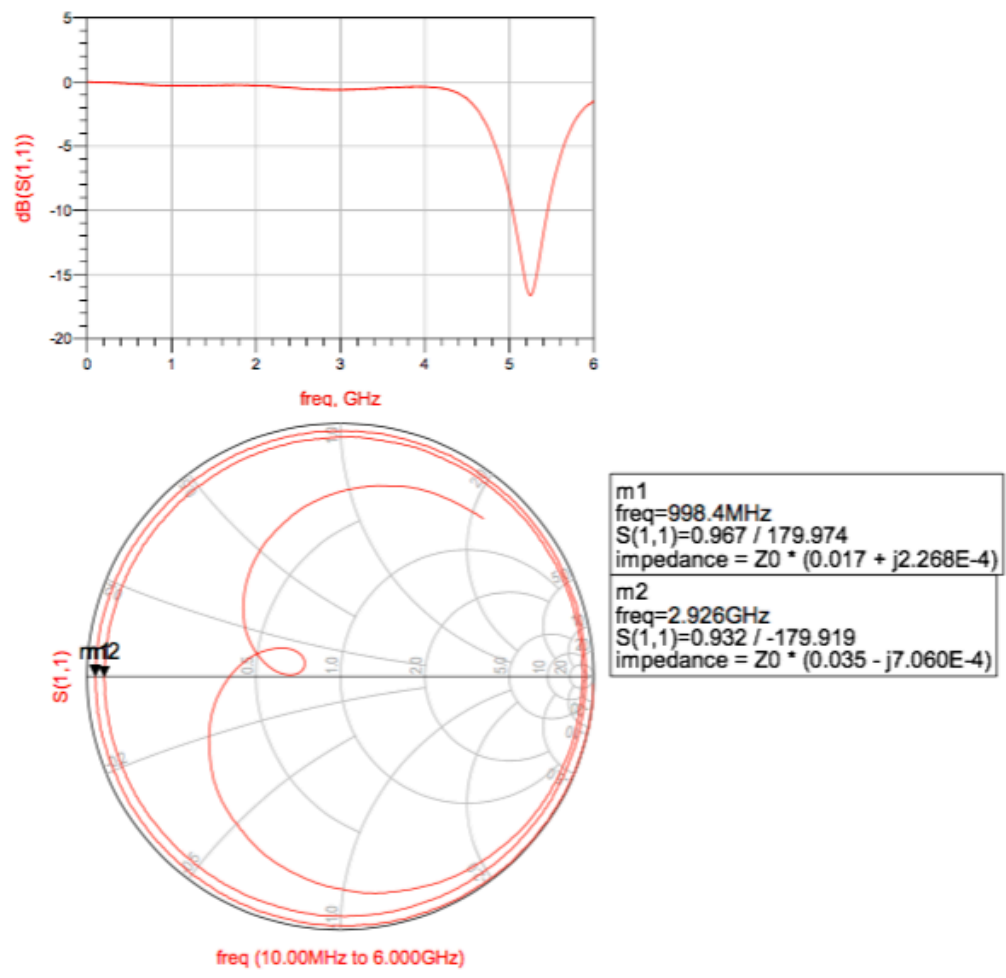

Figure 5.5: RF Coupon Unterminated S11

The assumed relative dielectric constant used for the design was 4.25 as specified in the Isola FR404 datasheet [56], which gave a calculated $50 \Omega$ line width of $122 \mathrm{mil}$. The effective dielectric constant, $\varepsilon_{\text {eff, }}$ can be calculated to be 3.235 using Eqn 5.1.

$$
\epsilon_{e f f}=\frac{\epsilon_{r}+1}{2}+\frac{\epsilon_{r}-1}{2}\left[1+12 * \frac{H}{W}\right]^{-1 / 2}
$$

Eqn 5.1 [57]

From the measurements in Figure 5.5, the unterminated RF coupon impedance is a short at $2926 \mathrm{MHz}$. Which means the $421 \mathrm{mil}$ trace is a multiple of a quarter wavelength at $2926 \mathrm{MHz}$. 
Based on an effective dielectric constant of 3.235 , the expected quarter wavelength at $2926 \mathrm{MHz}$ is $557 \mathrm{mil}$ as calculated in Eqn 5.2 .

$$
\lambda=\frac{v}{f}=\frac{c}{f \sqrt{\epsilon_{e f f}}}
$$

Eqn $5.2[57]$

Backwards calculating the effective dielectric constant for a 421 mil quarter wavelength at $2926 \mathrm{MHz}$ gives an $\varepsilon_{\text {eff }}$ of 2.397 . Given a height of $62 \mathrm{mil}$ for defined by the PCB stack up, this new $\varepsilon_{\text {eff }}$ gives an actual required trace width of 158 mil for a $50 \Omega$ microstrip line per Eqn 5.3.

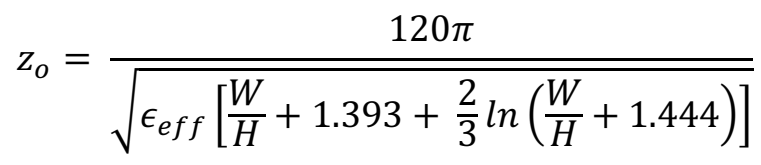

Eqn 5.3 [57]

The traces on the current PCB design have good performance up to $3 \mathrm{GHz}$. Above $3 \mathrm{GHz}$ the trace insertion loss increases significantly, especially around 4.5GHz. Additionally, at these high frequencies the impedance is not $50 \Omega$ and therefore the component performance may degrade due to poor terminations. To improve performance for this PCB stack up and process on the next design iteration, $158 \mathrm{mil}$ trace widths could be used instead of $121 \mathrm{mil}$ traces. The $158 \mathrm{mil}$ traces should be closer to $50 \Omega$ above $3 \mathrm{GHz}$. However, the wider traces will also will shift performance at lower frequencies where it is good currently, though this design is not intended to operate there.

\subsection{Transmitter Component Characterization}

Each of the components in the transmitter chain were characterized individually. Measured performance was compared to the datasheet performance specifications to determine if the part is behaving as expected when integrated in the system. 


\subsubsection{Sawtooth Amp}

The sawtooth amp was tested with a $100 \mathrm{kHz}$ sawtooth input waveform ranging from $0.8 \mathrm{~V}$ to $8.6 \mathrm{~V}$. The measured input and output waveforms are shown in Figure 5.6 below. Gain is calculated as Vout/Vin and shown in Figure 5.7.

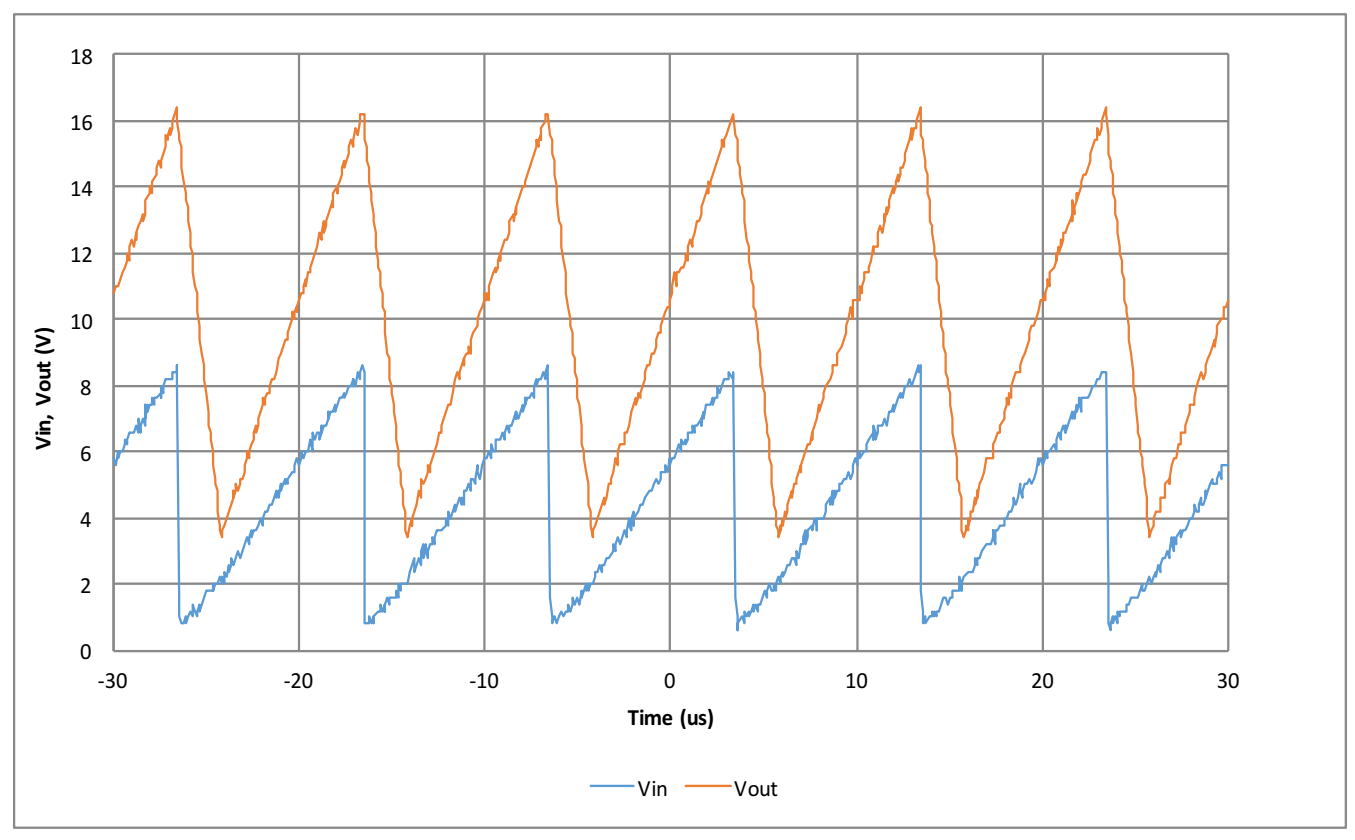

Figure 5.6: Sawtooth Amp Vin and Vout 


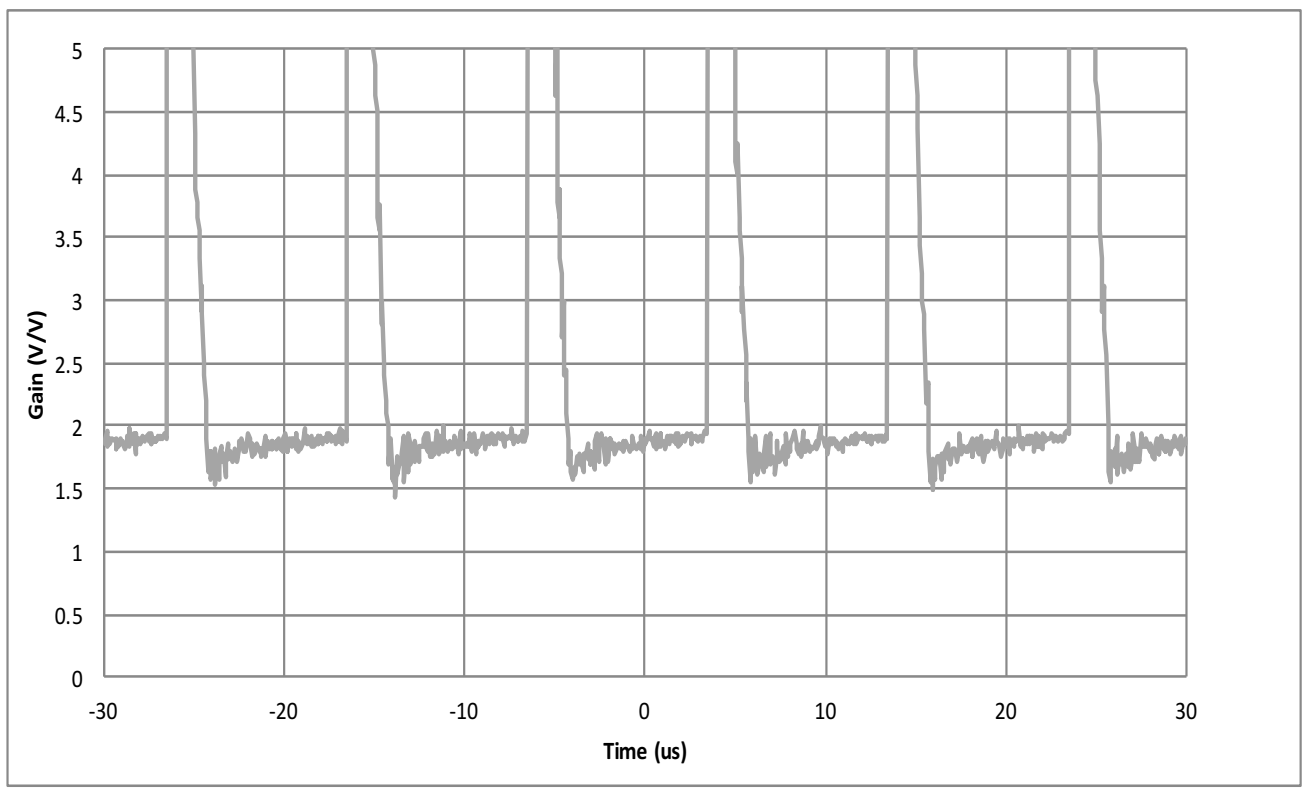

Figure 5.7: Sawtooth Amp Gain

The data above shows the voltage amplifier is working as expected. The gain is slightly below $2 \mathrm{~V} / \mathrm{V}$ for the entire rising edge. On the falling edge the slower slew rate of the op amp causes the output voltage to lag the input waveform, which causes high gain at those instances. This will not impact system operation, as the reflected system data during the rollover periods will be discarded regardless.

\subsubsection{Voltage Controlled Oscillator}

The voltage controlled oscillator frequency response versus input tuning voltage was tested by applying a constant input voltage to the VCO and monitoring the RF output on a spectrum analyzer. The results in Figure 5.8 below show a linear response as expected, with a required min and max voltages of $3.86 \mathrm{~V}$ and $16.20 \mathrm{~V}$ respectively to cover the entire $1.5-2.5 \mathrm{GHz}$ output range. 


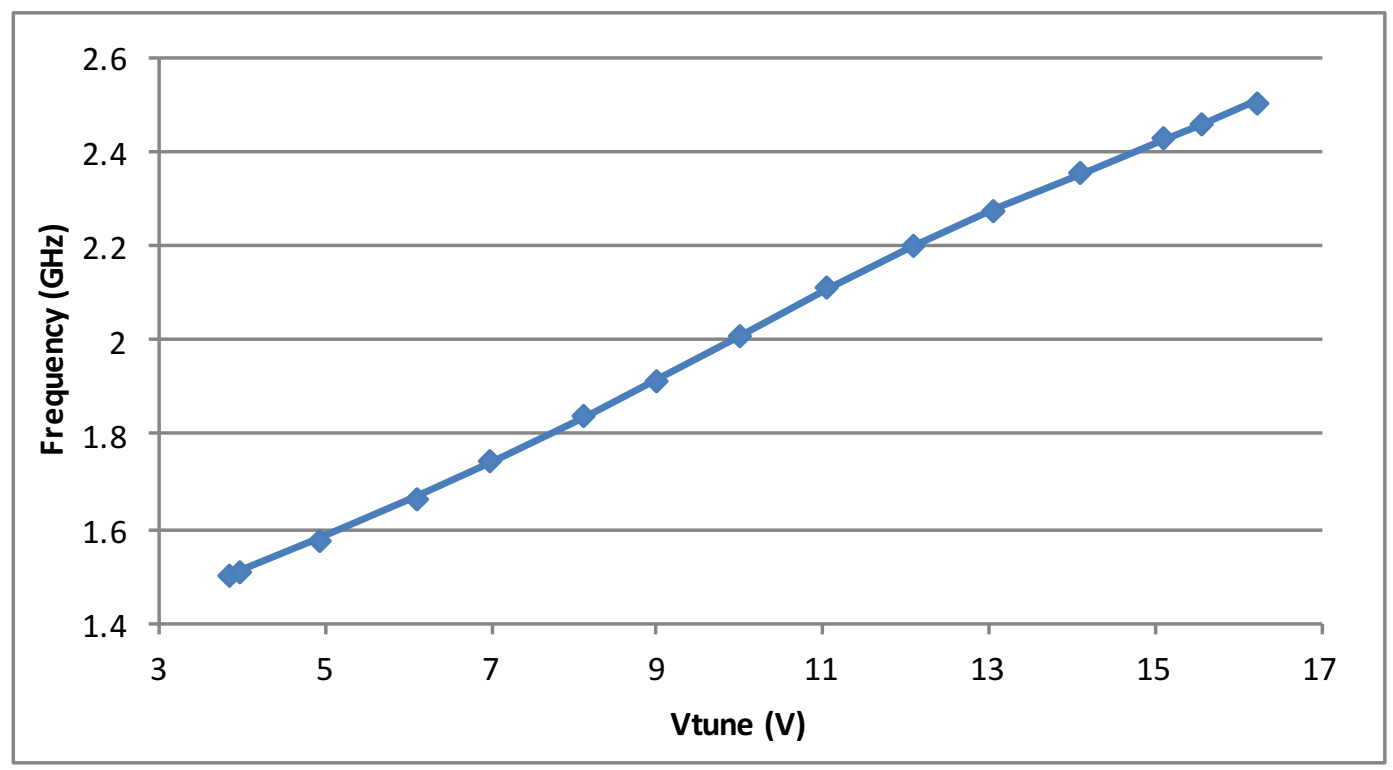

Figure 5.8: VCO Frequency Response

The fundamental RF output power of the VCO was measured at each Vtune data point along with the second and third harmonic power levels, shown below in Figure 5.9. The fundamental power is consistently around $3.5 \mathrm{dBm}$ across the entire tuning range. The harmonics vary across Vtune, with the worst case at $-14.7 \mathrm{dBm}$. All of these are in line with the datasheet performance.

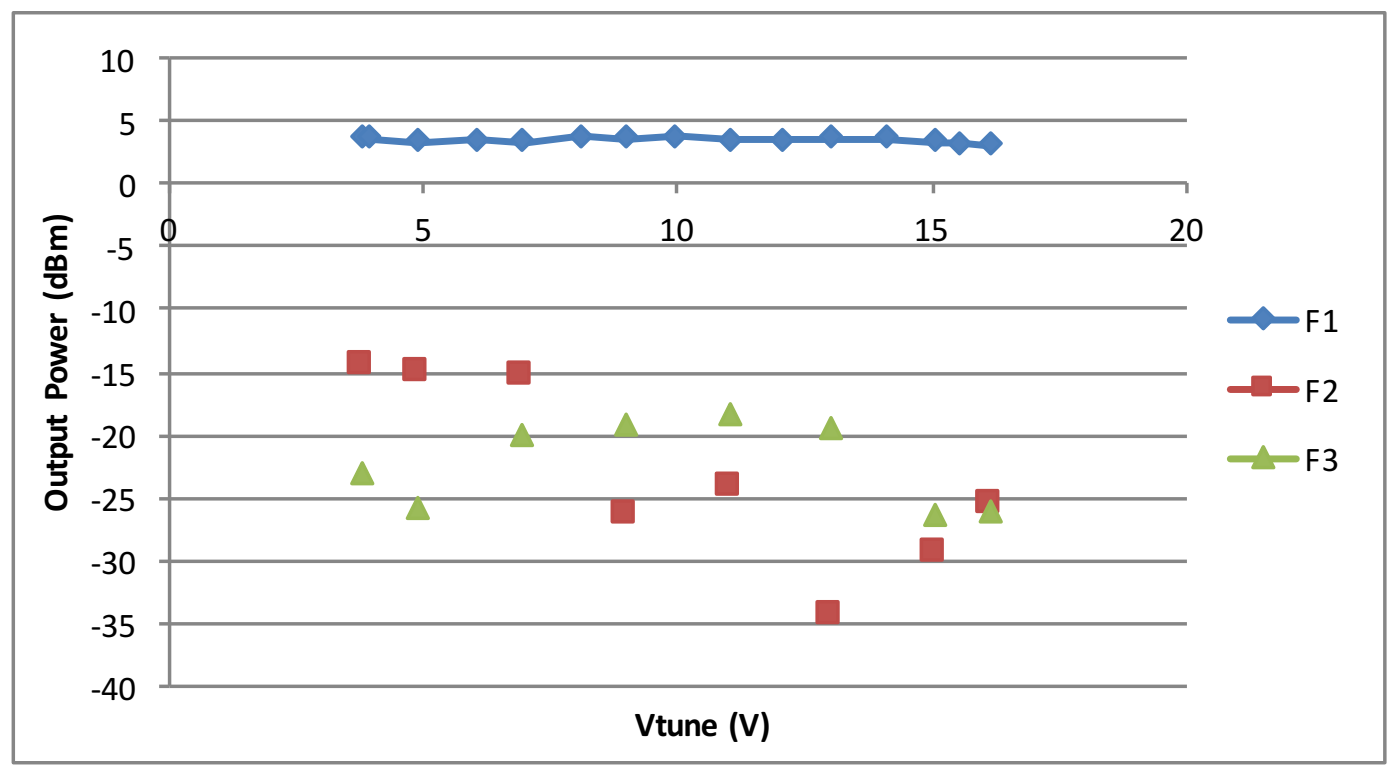

Figure 5.9: VCO Output Power 


\subsubsection{Low Frequency Tx Amplifier and Input Attenuator}

The input attenuator prior to the Low Frequency Tx Amplifier was targeting $4.5 \mathrm{~dB}$ attenuation to ensure the input power to the amplifier was in line with the output level required for the frequency mixer which follows. Figure 5.10 shows the attenuation ranges from 4.8$5.17 \mathrm{~dB}$ which is in line with the target attenuation plus trace loss. Figure 5.11 shows the return loss for the $50 \Omega$ attenuator which is better than $-20 \mathrm{~dB}$ for both the input and output, as desired.

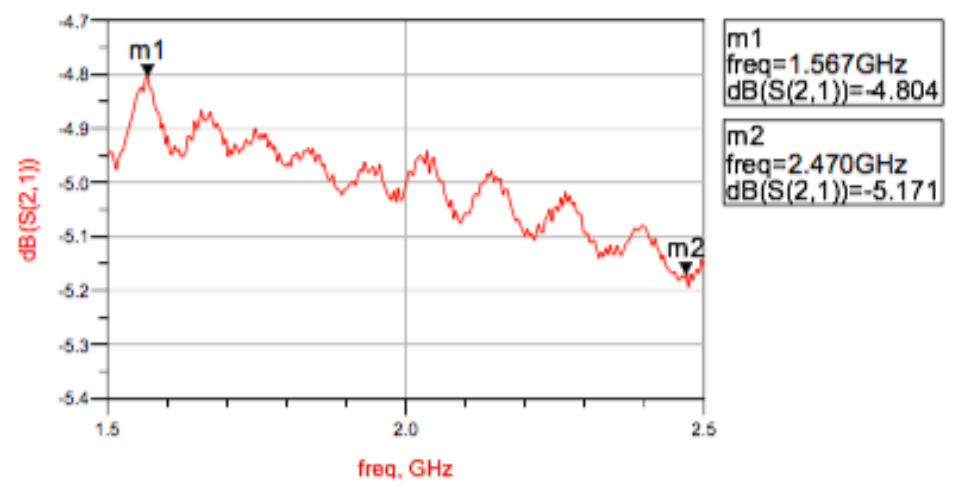

Figure 5.10: Input Attenuator Insertion Loss 

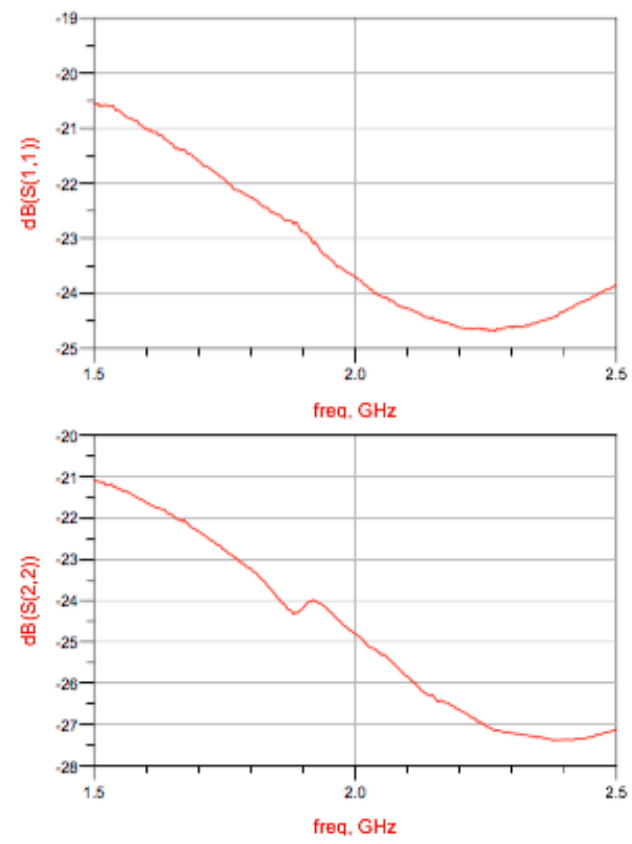

Figure 5.11: Input Attenuator Return Loss

The amplifier was also characterized across frequency for gain as well as input and output impedance. The gain is shown below in Figure 5.12, which is $14 \mathrm{~dB}$ at $1.5 \mathrm{GHz}$ and $9 \mathrm{~dB}$ at 2.5GHz. The gain at low frequencies is matching the expected amplifier performance, while the gain roll towards $2.5 \mathrm{GHz}$ is undesired.

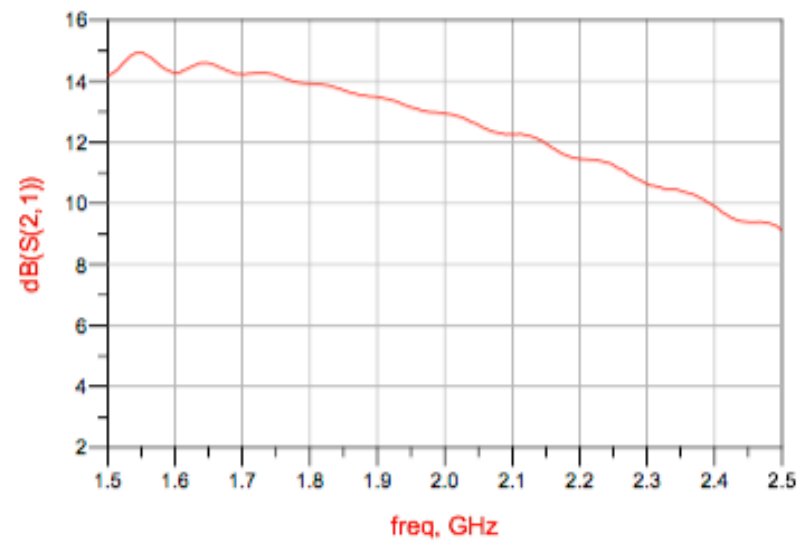

Figure 5.12: Low Frequency Tx Amplifier Gain

Figure 5.13 shows the input and output return loss for the amplifier. The measured input return loss at $1.5 \mathrm{GHz}$ has good performance at $11 \mathrm{~dB}$, but it degrades at higher frequencies hitting 
$3.5 \mathrm{~dB}$ at $2.5 \mathrm{GHz}$ which degrades the in-band performance. Figure 5.14 shows the input and output impedances across frequency on the smith chart, which corresponds to the return loss data.
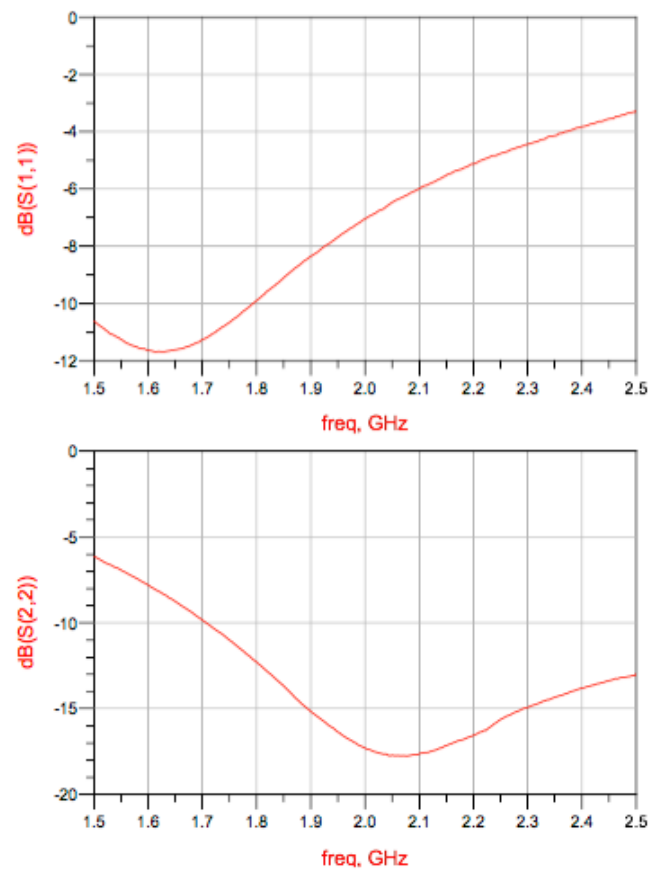

Figure 5.13: Low Frequency Tx Amplifier Return Loss
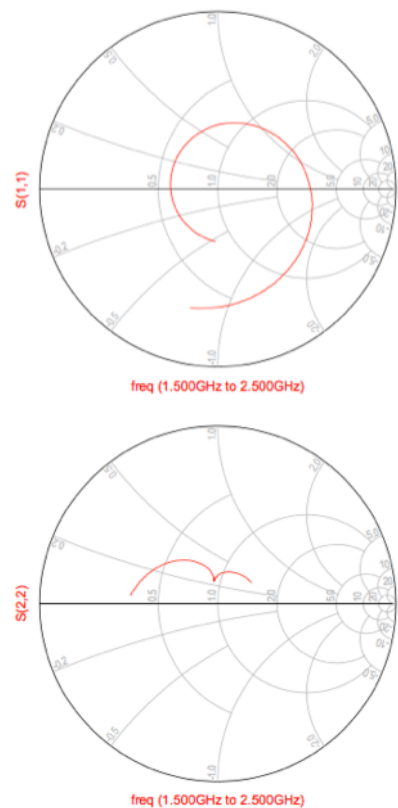

Figure 5.14: Low Frequency Tx Amplifier Input and Output Impedance 
The amplifier performance is good at the low end of the frequency range, matching the datasheet performance at $1.5 \mathrm{GHz}$. But the performance degrades at $2.5 \mathrm{GHz}$, seen by the drop in gain over frequency. The decreased gain at $2.5 \mathrm{GHz}$ corresponds to the input impedance which hits $3.5 \mathrm{~dB}$ of return loss; a poor input return loss of $3.5 \mathrm{~dB}$ equates to $2.57 \mathrm{~dB}$ of mismatch loss which is seen in the gain degradation. The system application for this component requires it to operate at a $50 \%$ fractional bandwidth. The impedance cannot be well matched across the wide frequency range of $1.5-2.5 \mathrm{GHz}$, leaving compromised performance across frequency.

\subsubsection{Frequency Multiplier}

The frequency multiplier was characterized across frequency for an input tone of $+10 \mathrm{dBm}$ which was swept from 1500-2500MHz. The desired second harmonic output level was recorded as well as the undesired fundamental, third, and fourth harmonic levels. The fourth harmonic is only measured up to $7 \mathrm{GHz}$, where the spectrum analyzer reached its frequency limit. The data is shown below in Figure 5.15.

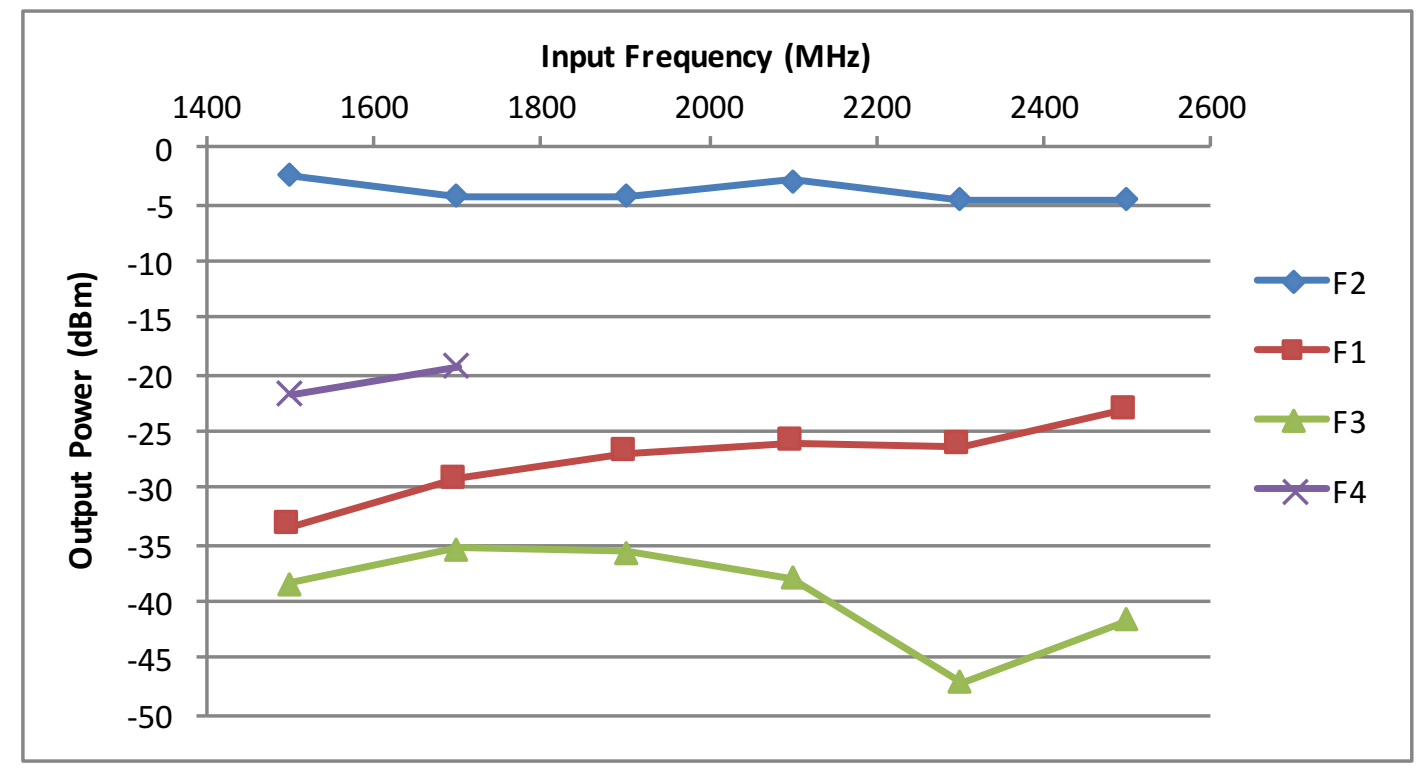

Figure 5.15: Frequency Multiplier Output 
The desired second harmonic output varies $\pm 1 \mathrm{~dB}$ across frequency, with the lowest power at $-4.5 \mathrm{dBm}$. This matches the datasheet conversion loss of $11.5-14.5 \mathrm{~dB}$ for the $10 \mathrm{dBm}$ input tone used here. The fundamental leakage is better than $-23.1 \mathrm{dBm}$ across the desired frequency range. The worst case third and fourth harmonic respectively is $-35.4 \mathrm{dBm}$ and $-19.2 \mathrm{dBm}$. All of these are better than the typical datasheet specified harmonic output of $22 \mathrm{dBc}$, $28 \mathrm{dBc}$, and $17 \mathrm{dBc}$ respectively.

\subsubsection{Band Pass Filter}

Wideband sparameters were measured for the band pass filter. Figure 5.16 below shows the filter response across frequency up to $6 \mathrm{GHz}$. The bandpass filter shape can be seen with the passband between 3-5GHz. The wideband plot shows the out of band attenuation is better than $25 \mathrm{~dB}$ below $2.5 \mathrm{GHz}$ and above $5.5 \mathrm{GHz}$, which is in line with the filter specification.

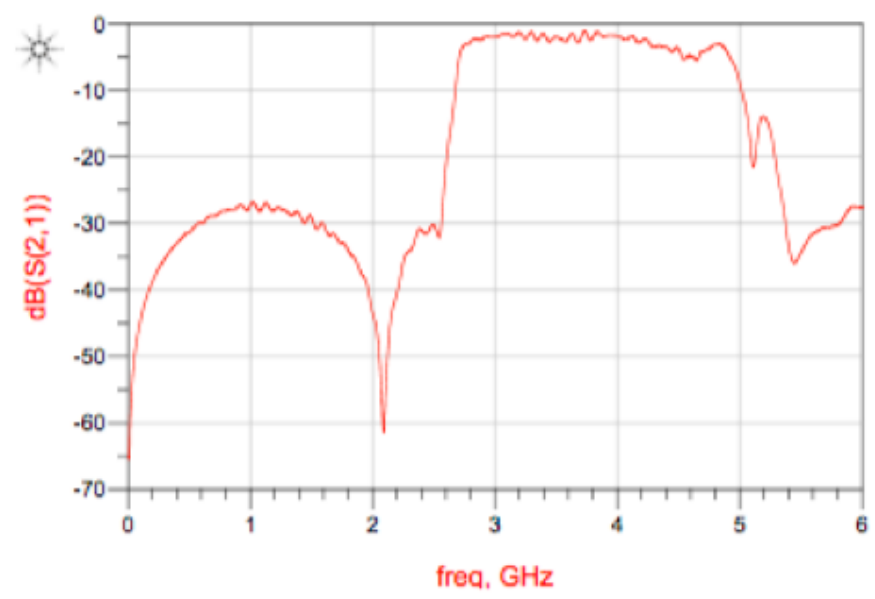

Figure 5.16: BPF Wideband Attenuation 


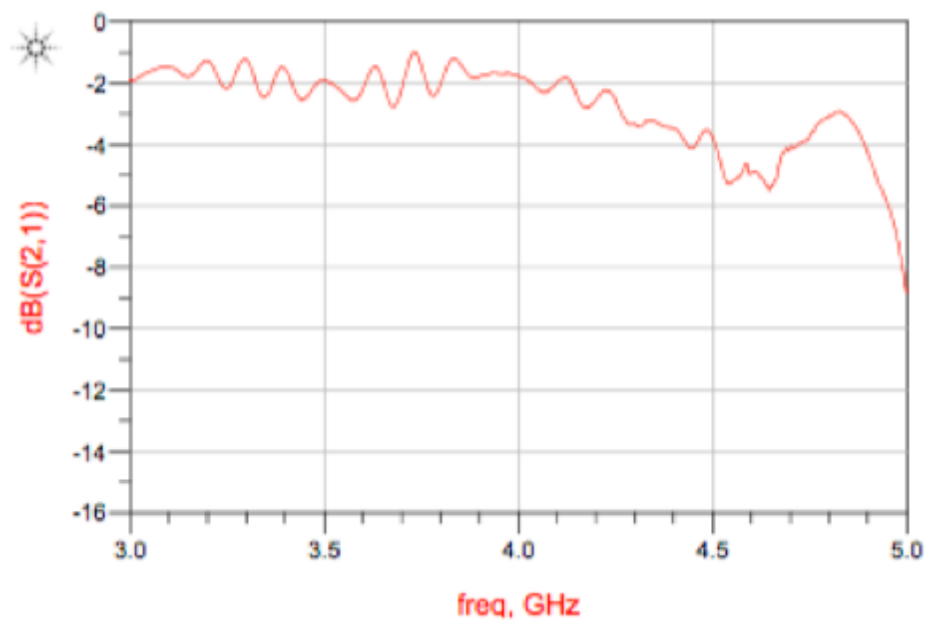

Figure 5.17: BPF Insertion Loss

Figure 5.17 shows the in-band insertion loss of the filter. The insertion loss is in line with the filter spec after accounting for the additional PCB trace loss. The response shows around $2 \mathrm{~dB}$ insertion loss up to $4 \mathrm{GHz}$, and increased loss above $4 \mathrm{GHz}$ which is due to the $\mathrm{PCB}$ trace response which starts to degrade above that frequency. The highest system frequency is 4.9 GHz and the filter component passband is specified up to $4752 \mathrm{MHz}$, so the insertion loss roll off approaching $5 \mathrm{GHz}$ is expected.

\subsubsection{Microstrip Stepped Impedance LPF}

The microstrip stepped impedance low pass filter frequency response was captured with a VNA and the results are shown in Figure 5.18. The filter does not show the intended response of a 5.5GHZ low pass filter, as intended by the design and confirmed with initial ADS simulations. 


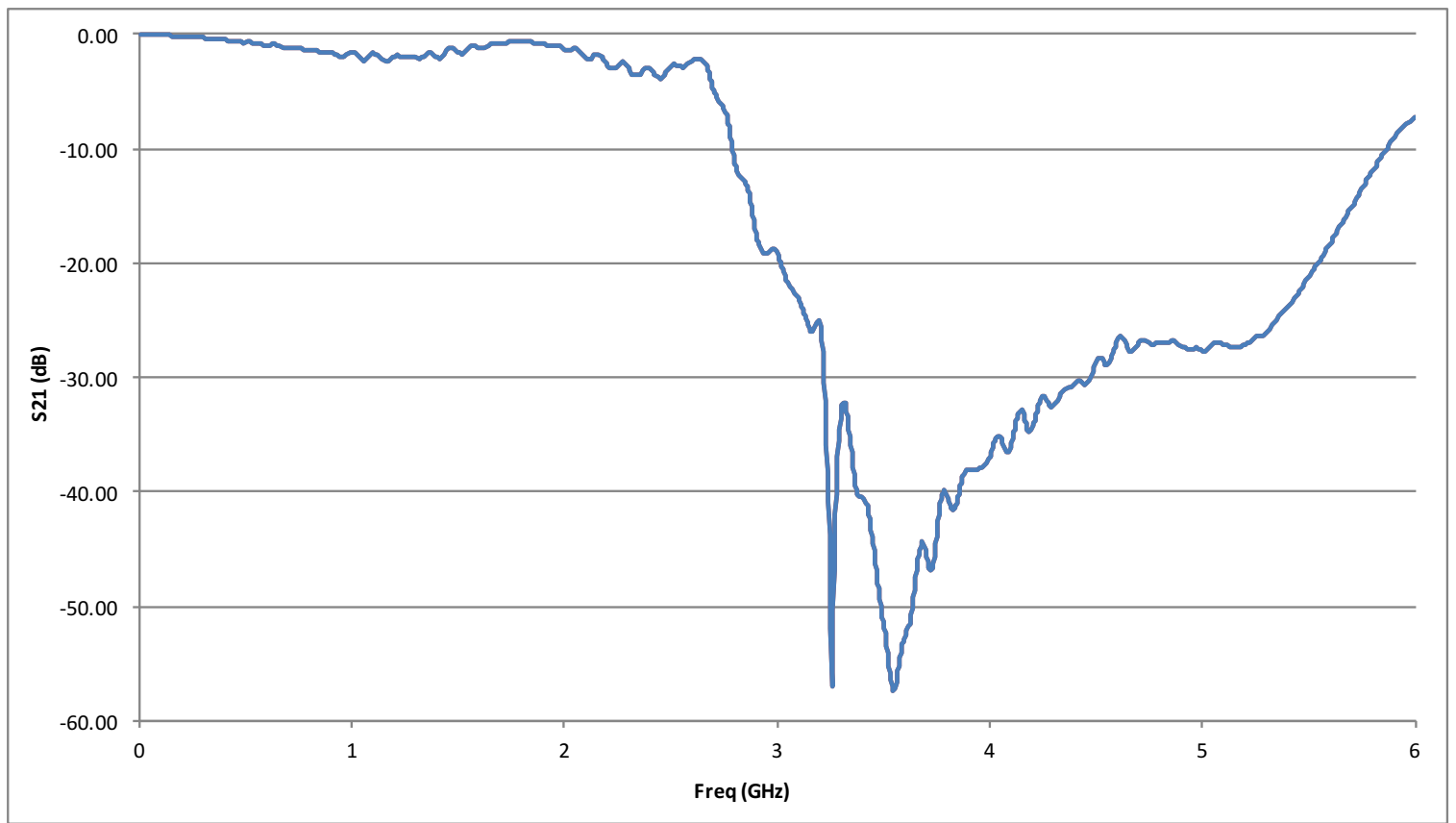

Figure 5.18: Microstrip Stepped Impedance LPF Measured Frequency Response

The ADS simulation used to verify the design was the piecewise schematic sparameter simulation tool. It uses a model for each individual component of the design and cascades the responses together. In order to verify if the unexpected measured response of the microstrip filter is due to its design or if it is related to the filter integration on the $\mathrm{PCB}$, the filter design was resimulated using ADS Momentum 3D Planar EM Simulator. ADS Momentum uses a frequencydomain Method of Moments technique to accurately simulate electromagnetic effects of passive circuits including coupling and parasitics [58]. The ADS Momentum simulation setup for the schematic and layout are shown below in Figure 5.19. 


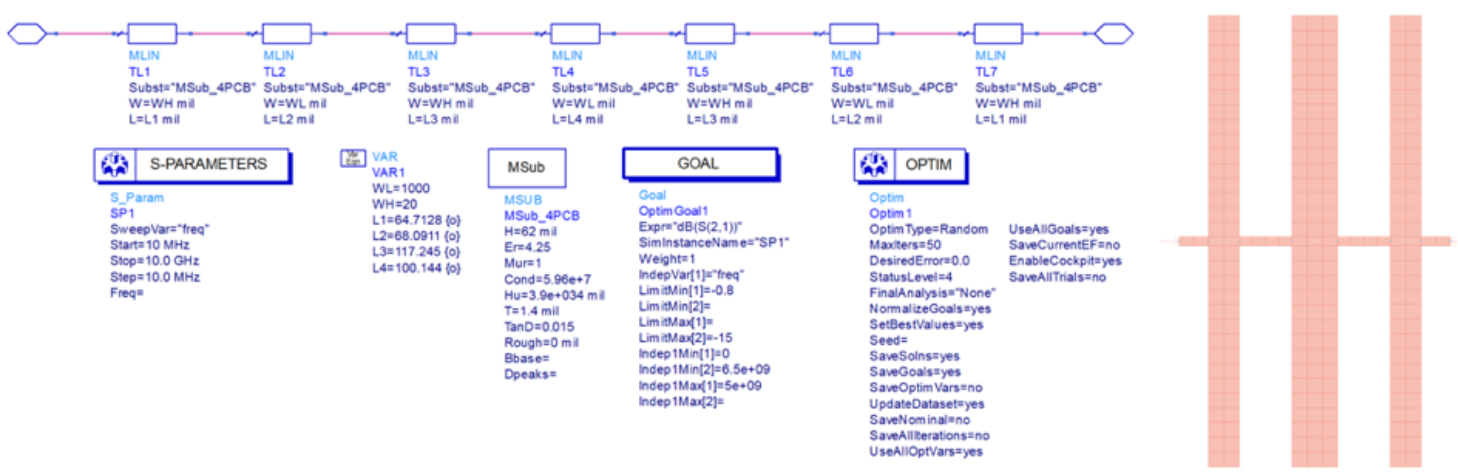

Figure 5.19: Microstrip Filter Momentum Simulation

The Momentum simulated frequency response is shown in Figure 5.20. By comparing this simulation to Figure 5.18 above, the filter measured response matches the 3D EM simulation.
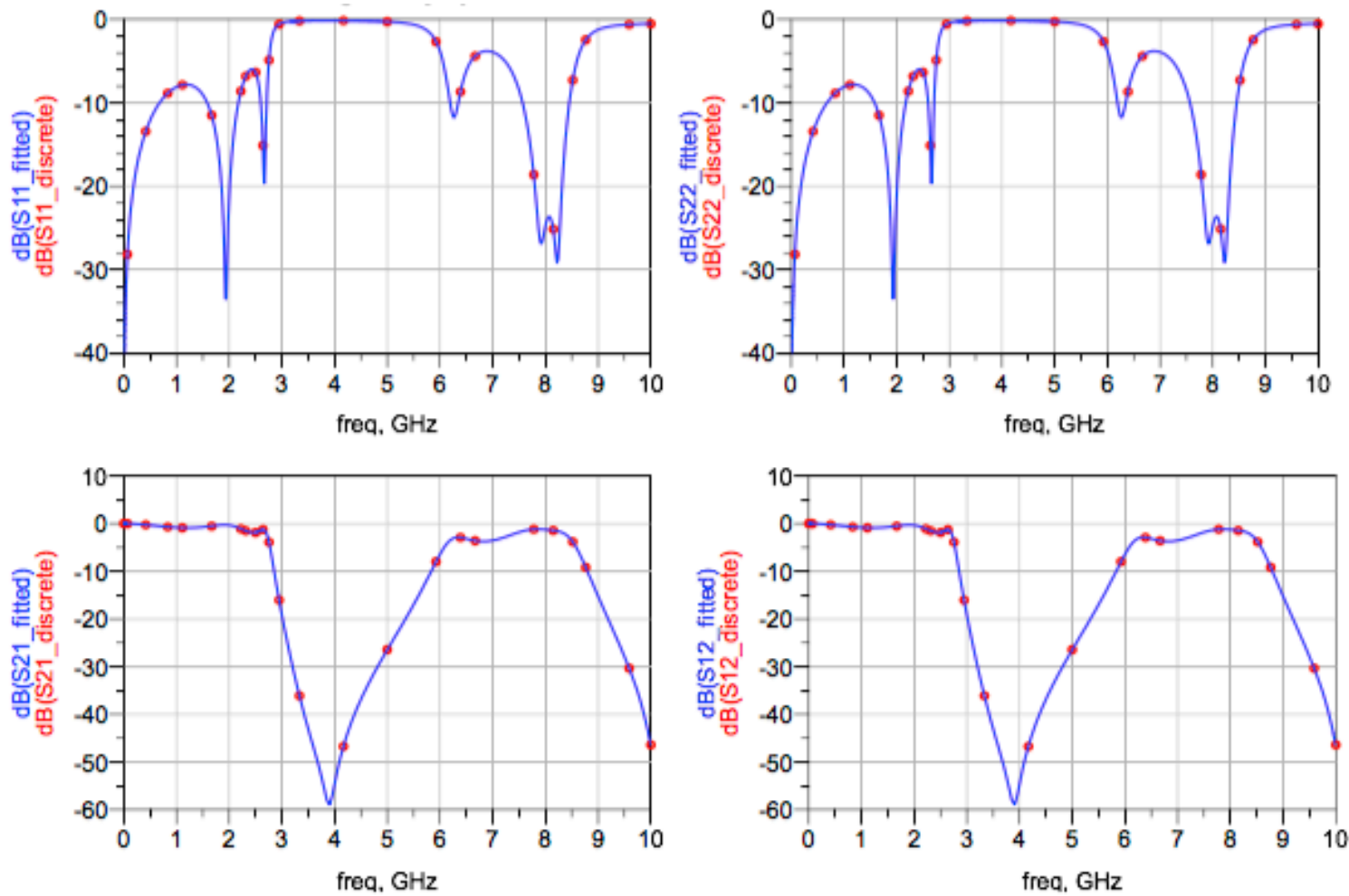

Figure 5.20: Microstrip Filter Momentum Simulation Frequency Response

At high frequencies and when the microstrip sections have a width much greater than length, the coupling between filter arms cannot be ignored, as confirmed by comparing the piecewise ADS simulations used in the filter design versus the 3D planar EM Momentum 
simulations. To get a microstrip stepped impedance low pass filter with a cutoff frequency of $5.5 \mathrm{GHz}$, the filter would need to be redesigned to account for this coupling.

\subsubsection{Tx Gain Block Amplifier}

The Tx Gain Block amplifier was characterized over frequency, and the results for gain are shown in Figure 5.21. There is a resonance at $4.4 \mathrm{GHz}$ where the gain hits $-5 \mathrm{~dB}$, and the gain drops across frequency down to $10 \mathrm{~dB}$ at $5 \mathrm{GHz}$. At low frequency the amplifier is achieving the desired gain near $15 \mathrm{~dB}$, but performance is degraded across the desired operating band of 3.1$4.9 \mathrm{GHz}$.
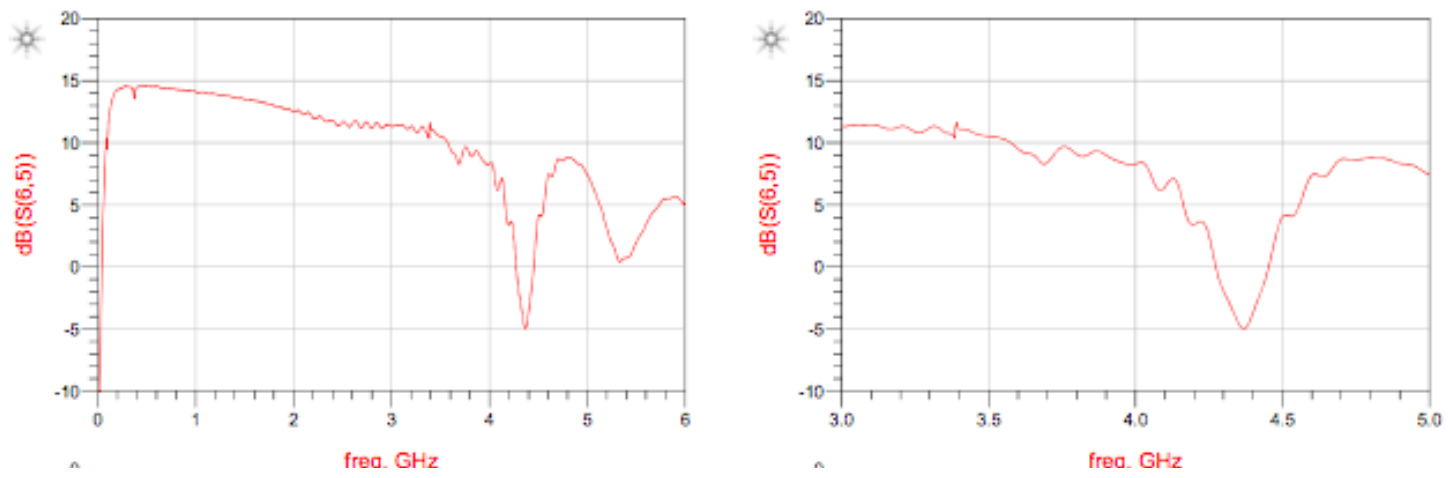

Figure 5.21: Tx Gain Block Amplifier Gain

Figure 5.22 shows the input and output impedance as well as return loss for the Tx Gain Block amplifier. The impedance contour is widespread, especially on the output, and return loss at high frequencies is poor. The resonance seen at $4.4 \mathrm{GHz}$ in the gain measurement is also seen in the output impedance, where the return loss reaches $2.5 \mathrm{~dB}$. 

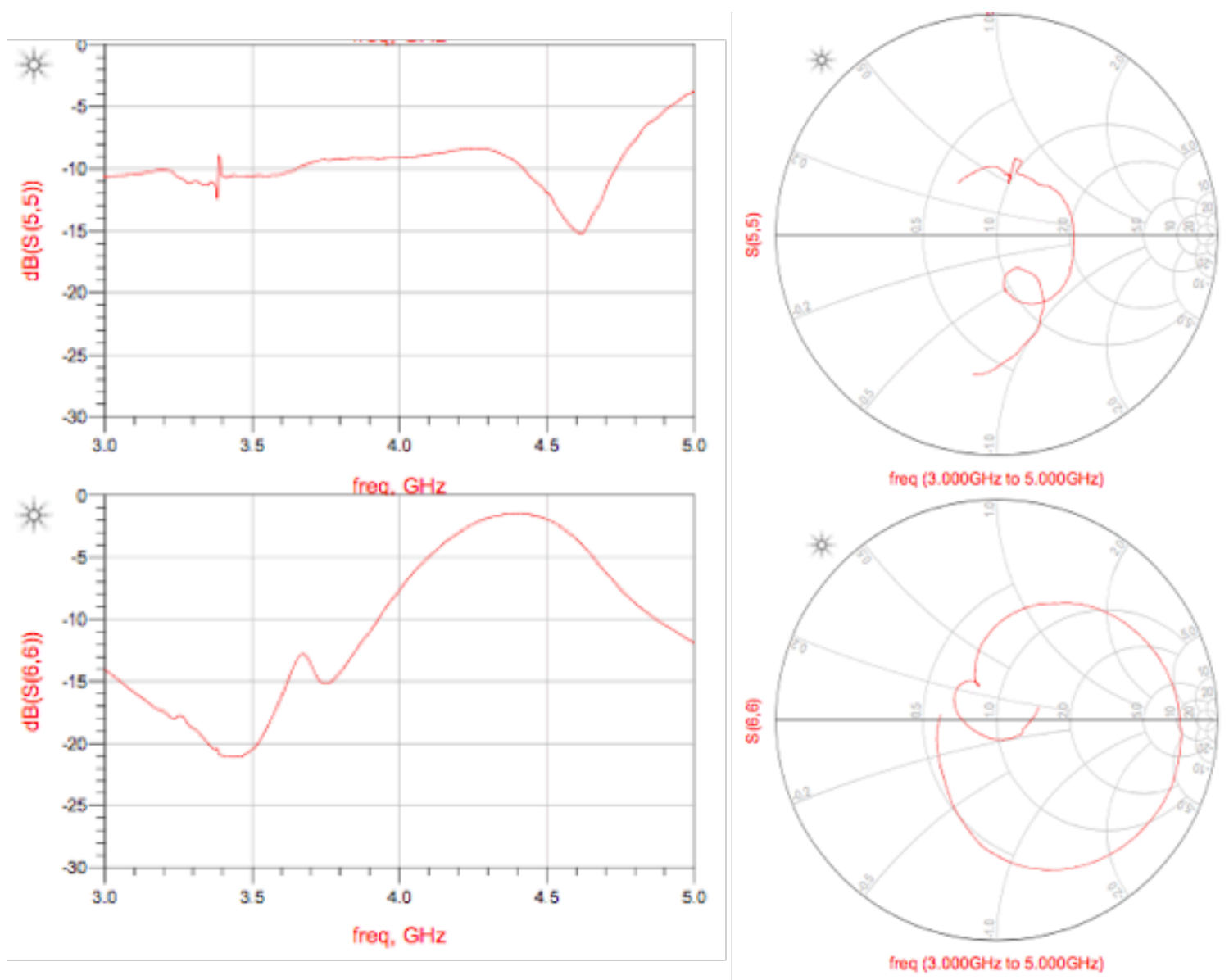

Figure 5.22: Tx Gain Block Amplifier Return Loss and Impedance

The characteristics of the Tx Gain Block Amplifier are similar to what is seen in the other amplifiers. Sections 5.3.8 and 5.4.3 discuss improvements made to the amplifiers to address the resonance, improve the return loss, and increase the gain at higher frequencies.

\subsubsection{Tx Power Amplifier}

The Tx Power Amplifier was characterized over frequency. Figure 5.23 below shows the wideband gain up to $6 \mathrm{GHz}$ as well as the in-band gain. 

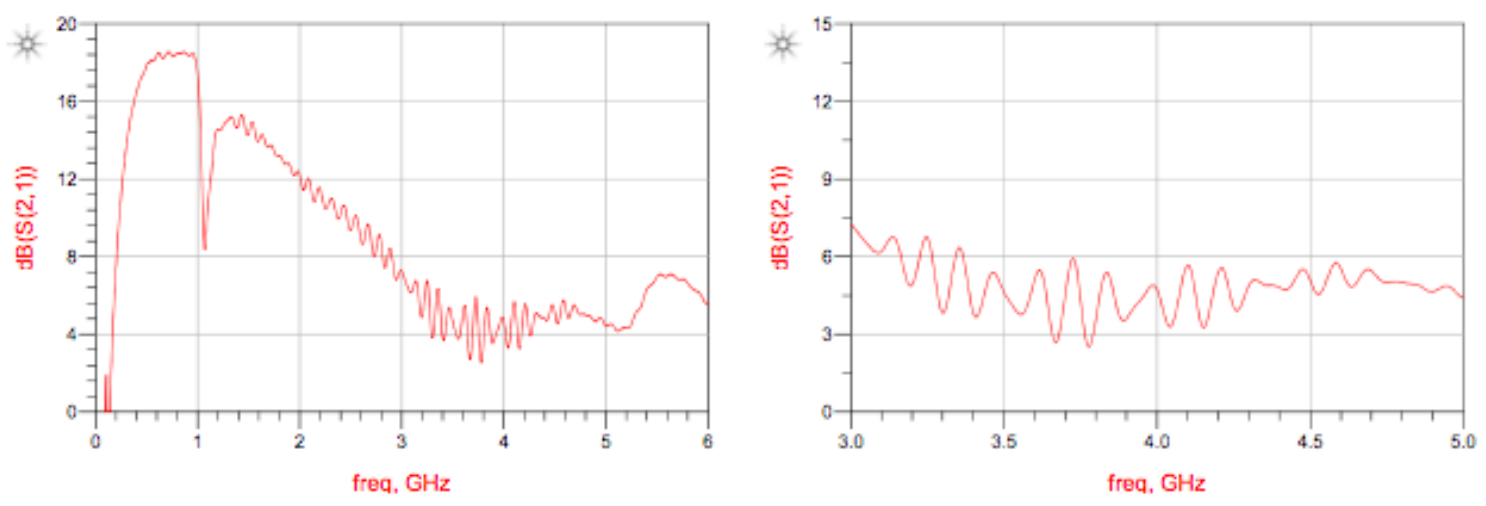

Figure 5.23: Tx PA Gain

There is a sharp resonance at $1.1 \mathrm{GHz}$, and the gain drops across frequency until reaching 3-6dB across the passband. The $1.1 \mathrm{GHz}$ resonance was fixed by placing a $1.3 \mathrm{uH}$ wideband $\mathrm{RF}$ Choke on the amplifier bias line to provide high impedance isolation at RF frequencies.

APPENDIX E: 1.3uH Wideband Bias Choke discusses the details of the RFC that was selected. Figure 5.24 shows a comparison of the Tx Power Amplifier gain before and after adding the 1.3uH RFC.
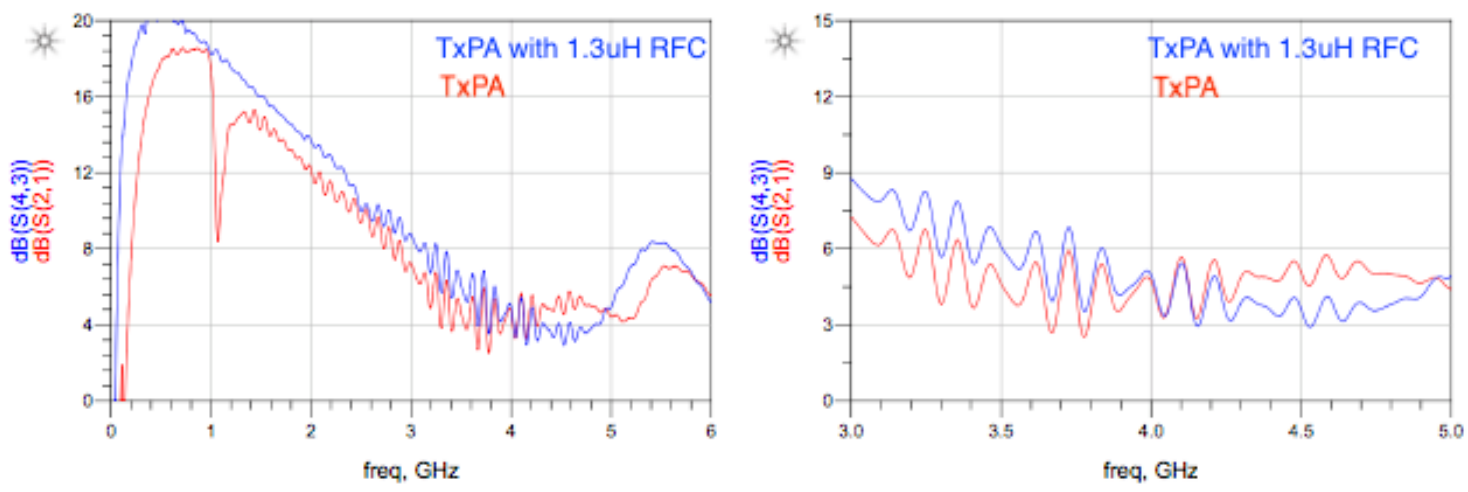

Figure 5.24: TxPA Gain vs 1.3uH RFC

The reference plane for the above measurements was at the $0 \Omega$ resistors placed on the input and output of the amplifier, which have reference designators of 0-101 and 0-104. Because the PCB traces have poor performance at high frequency, as discussed in Section 5.2, the measurement was repeated at the matching component locations L103 and L104. This decreased 
the length of the PCB traces included in the measurement. Figure 5.25 compares the Tx PA gain at the two different measurement points. The gain at $5 \mathrm{GHz}$ increases by $5.5 \mathrm{~dB}$, while the low frequency gain is relatively unchanged.
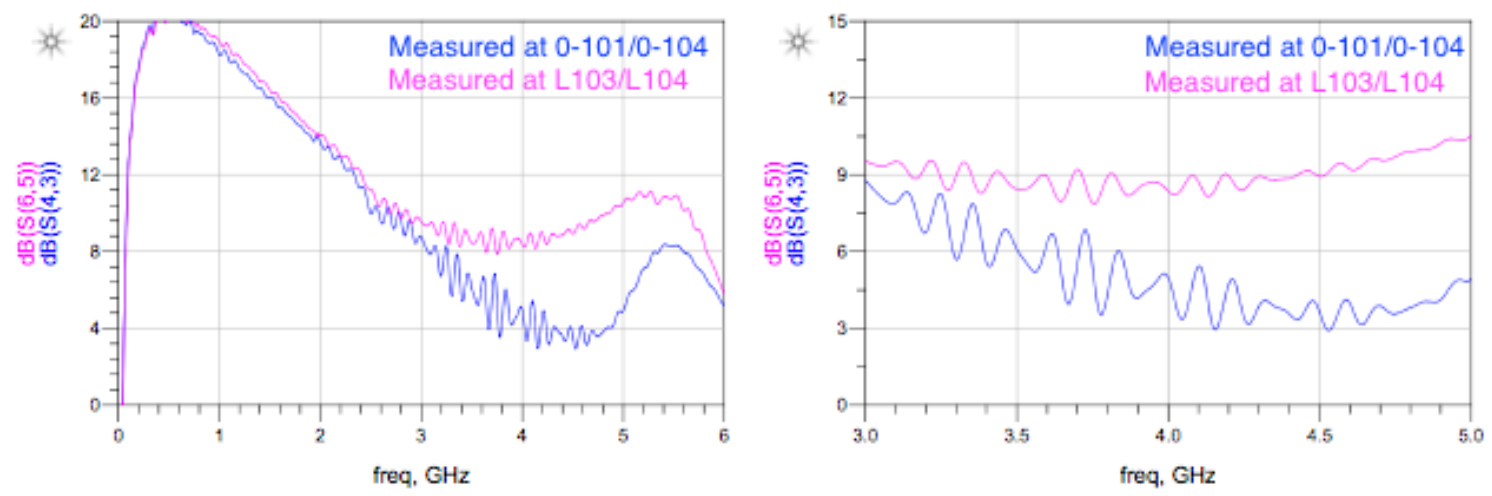

Figure 5.25: TxPA Gain vs Measurement Location

Figure 5.26 and Figure 5.27 show the Tx PA input and output impedance and return loss at the two measurement points. Measuring at the matching components with smaller PCB traces greatly improves the output impedance, with an average return loss improvement of $7 \mathrm{~dB}$ across the passband. This improvement reduces the mismatch loss of the signal, and allows the $50 \Omega$ PA to operate closer to its designed impedance for gain and power contours.

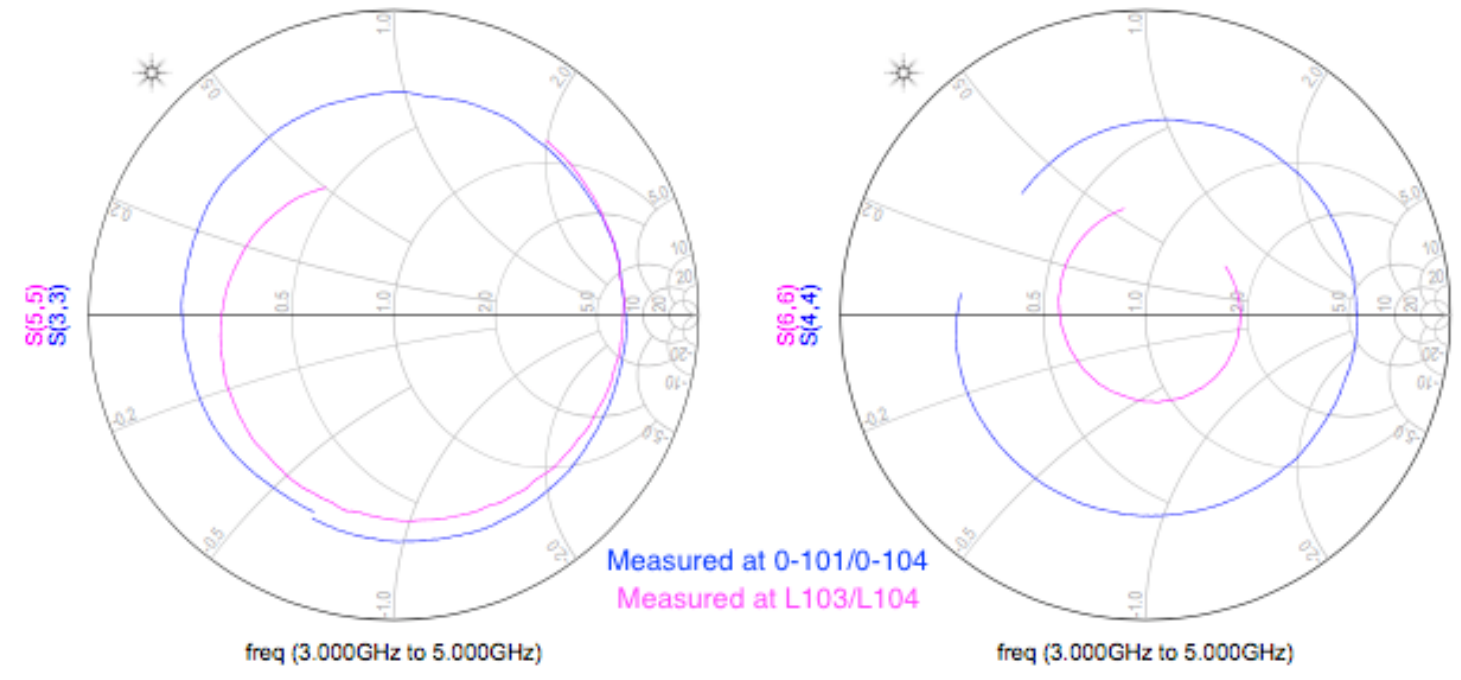

Figure 5.26: TxPA Impedance vs Measurement Location 

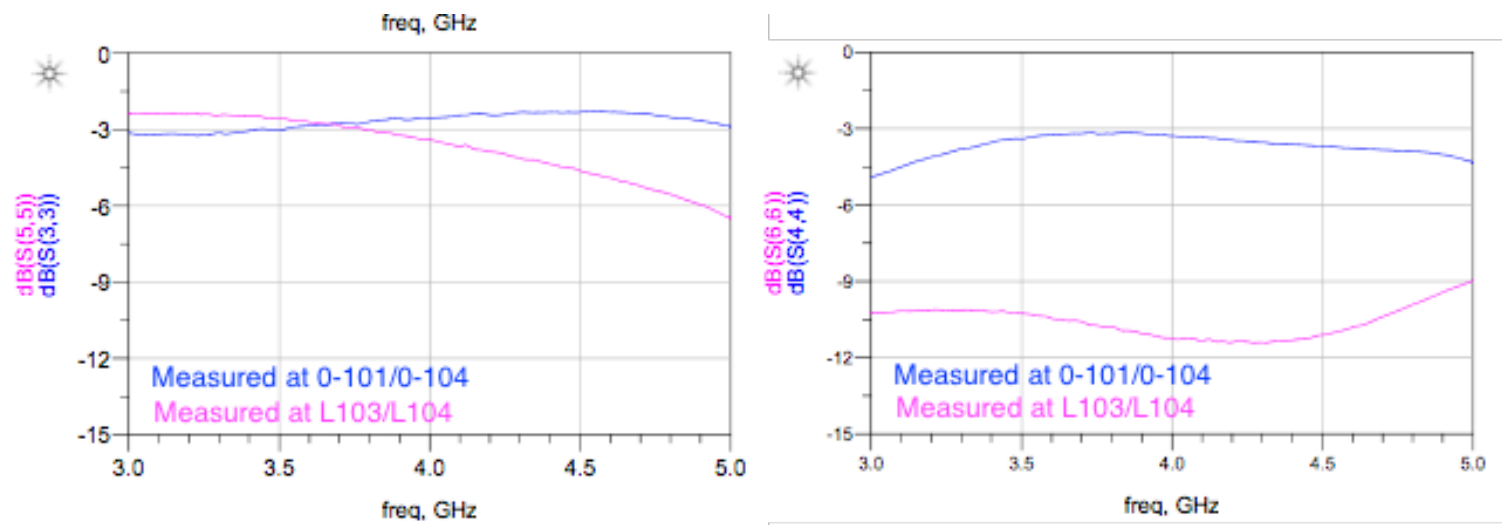

Figure 5.27: TxPA Return Loss vs Measurement Location

\subsubsection{Power Splitter}

The power splitter was not successfully soldered to the PCB to allow for characterization. Neither reflow oven or manually soldering with a heat gun were able to make clean connections on each signal pin. All characterization data showed large amounts of isolation through all splitter signal paths. Potentially a new component with a different package style but similar RF parametrics could be chosen instead of the Mini Circuits GP2X+, which may allow for easier soldering to the PCB. Alternatively, higher precision soldering methods would need to be used for this component.

\subsubsection{Coupler}

The coupler performance is shown below in Figure 5.28 and Figure 5.29, which depict the in-band insertion loss of the through path and the coupling factor of the coupled path respectively. 


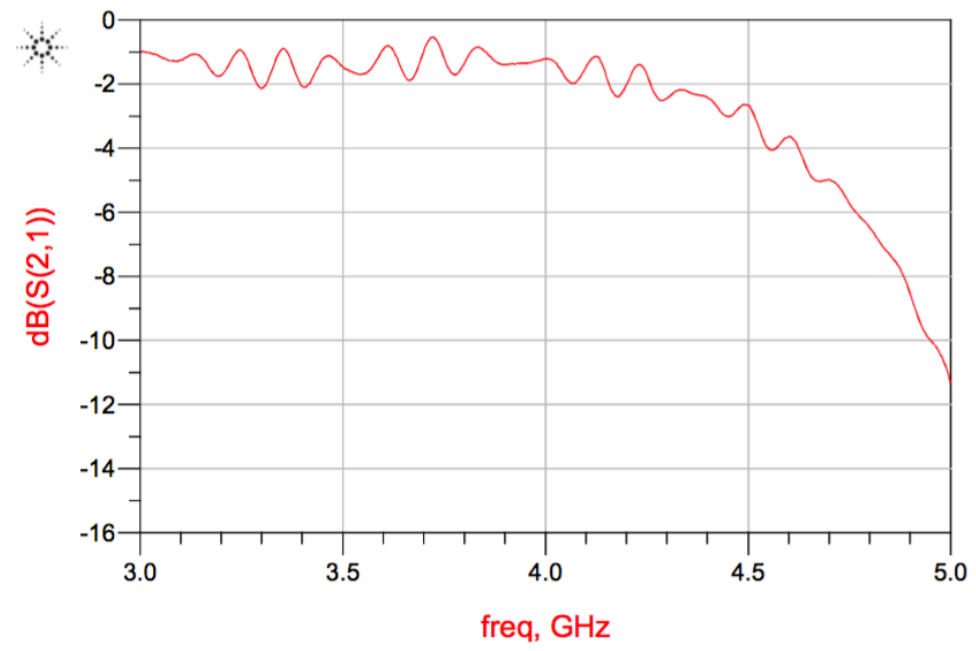

Figure 5.28: Coupler Insertion Loss

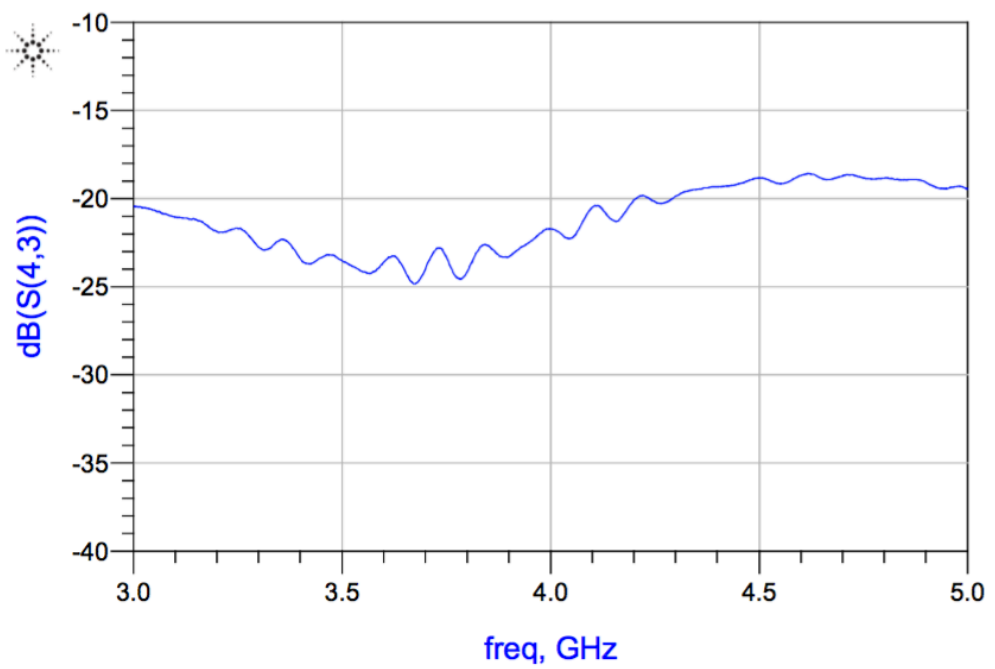

Figure 5.29: Coupler Coupling Factor

The coupler insertion loss is in line with the specification of $0.7 \mathrm{~dB}$ max up to $4.5 \mathrm{GHz}$ after accounting for the additional loss due to the PCB traces. The loss degrades significantly above $4.5 \mathrm{GHz}$, where the trace loss increases. Additionally, the impedance deviates from $50 \Omega$ at those frequencies which could cause additional degradation in the coupler performance. The coupling factor specification varies from $15-18.2 \mathrm{~dB}$ across frequency with the highest coupling 
factor between 4.5-5GHz. Again, the measurement data aligns with this performance with a slightly lower coupling factor due to the added trace loss.

\subsubsection{Output Power Attenuator}

The output power attenuator calculations show that the nominal attenuation should be 41.5dB. Figure 5.30 below shows the measured attenuation of the circuit, which has a nominal attenuation of about $18 \mathrm{~dB}$. The high target attenuation is not achieved due to the limited isolation at high frequencies between the components which have close physical spacing on the PCB. To address this, the resistive PI network would have to have increased physical spacing in the layout, or multiple lower value attenuators could be cascaded. Another option is to modify the architecture design to not require such large attenuation of the transmit signal.

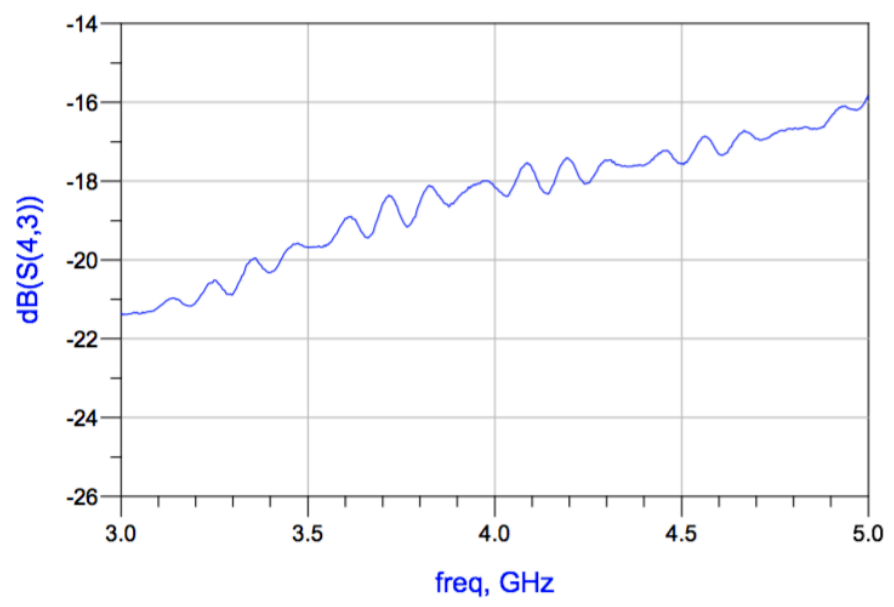

Figure 5.30: Output Power Attenuator S21

\subsection{Receiver Component Characterization}

Each of the components in the receiver chain were characterized individually. Measured performance was compared to the datasheet performance specifications to determine if the part is behaving as expected when integrated in the system. 


\subsubsection{Band Pass Filter}

The Rx Band Pass Filter is identical to the one used in the Tx chain. See section 5.3.5 for data and analysis.

\subsubsection{Low Noise Amplifier}

The low noise amplifier was characterized over frequency. Initial measurements show sharp gain roll off below $1.9 \mathrm{GHz}$, and low gain at $5 \mathrm{GHz}$. Similar to the Tx PA which was discussed in Section 5.3.8, both of these are improved by adding a 1.3uH RFC and moving the measurement plane to the LNA matching, which reduces the PCB trace length included in the measurement.

Figure 5.31 shows the LNA gain across frequency for the initial measurement compared to the $1.3 \mathrm{uH}$ RFC and shifted measurement plane. This removes the gain drop at low frequencies, and increases the gain at $4.5 \mathrm{GHz}$ by $6 \mathrm{~dB}$. The amplifier performance with the $1.3 \mathrm{uH}$ RFC is good at low frequency. Additional gain is desired at $5 \mathrm{GHz}$ where it has rolled off to $4 \mathrm{~dB}$.
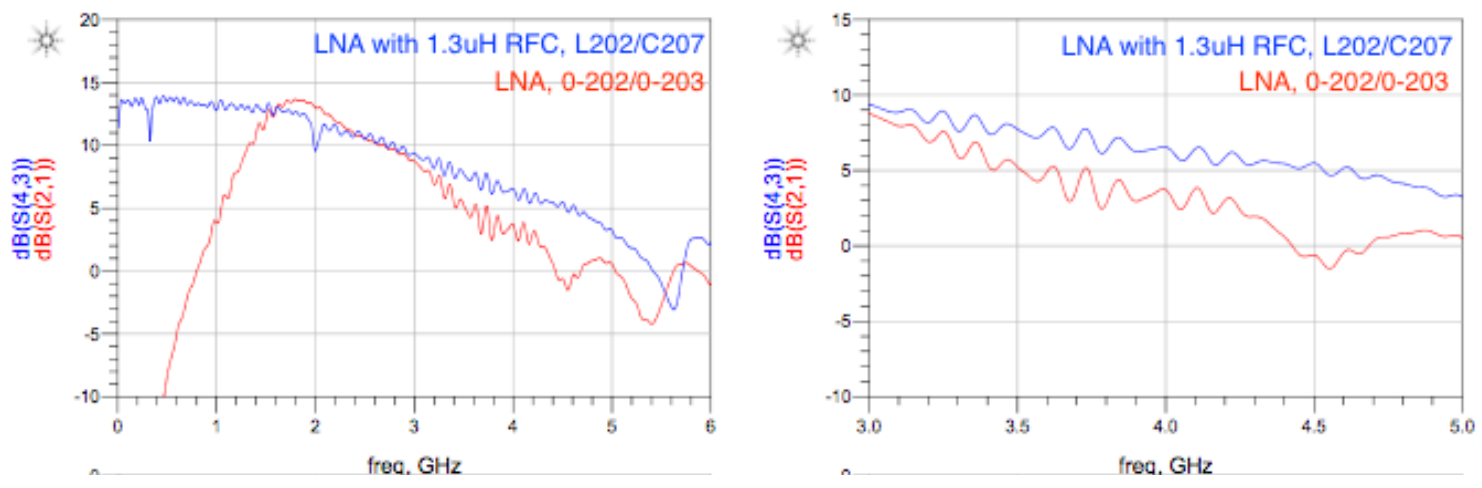

Figure 5.31: LNA Gain with 1.3uH RFC and Measurement Location

Figure 5.32 and Figure 5.33 show the LNA input and output impedances as well as return loss for the two measurement cases. Both the input and output impedances are improved, with the input return loss improving to better than $10 \mathrm{~dB}$ up to $4.3 \mathrm{GHz}$. 


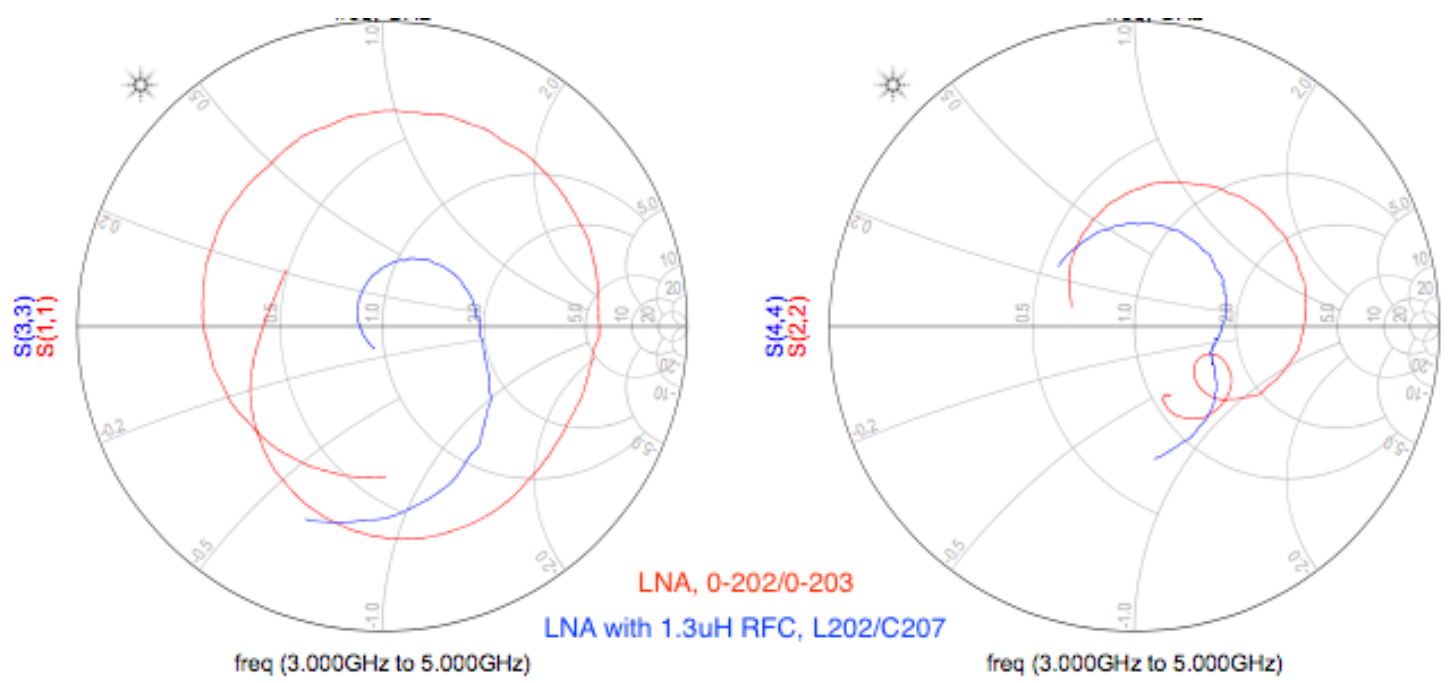

Figure 5.32: LNA Impedance with 1.3uH RFC and Measurement Location
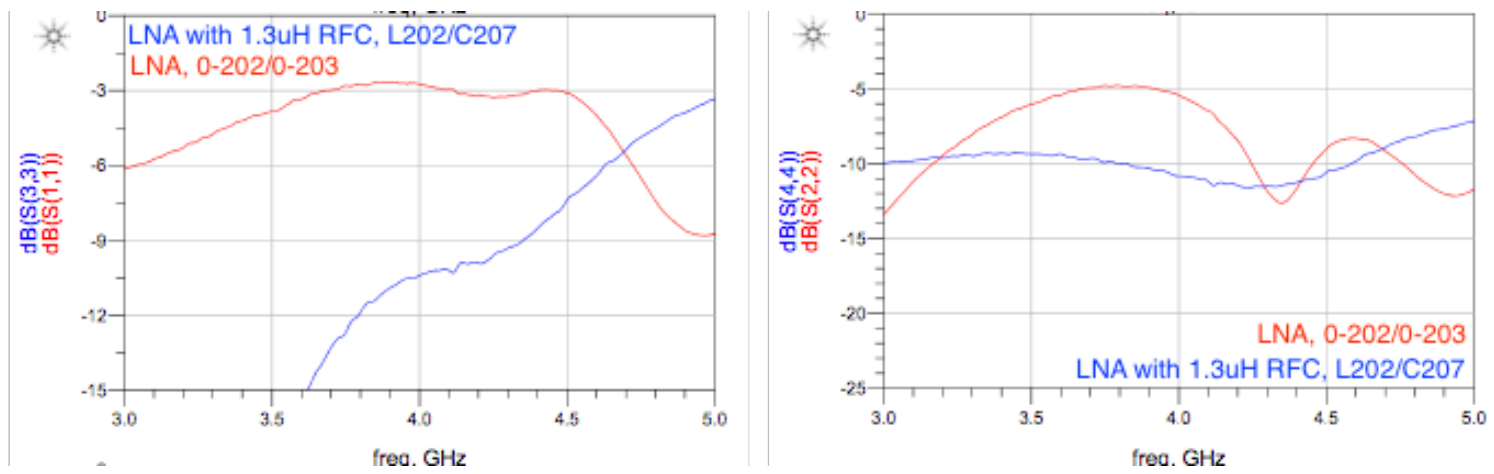

Figure 5.33: LNA Return Loss with 1.3uH RFC and Measurement Location

\subsubsection{Rx Gain Block}

The initial characterization of the $\mathrm{Rx}$ Gain Block amplifier has a resonance at $4.5 \mathrm{GHz}$ which causes the gain to drop down to $0 \mathrm{~dB}$. A $1.3 \mathrm{uH}$ RFC is added to the amplifier to improve $\mathrm{RF}$ isolation to the bias network. The gain across frequency is shown in Figure 5.34 which compares the initial results to the improvement with the 1.3uH RFC. With the added RFC the amplifier meet expected performance at low frequencies, and the resonance at $4.5 \mathrm{GHz}$ is removed. Gain at $5 \mathrm{GHz}$ is $7 \mathrm{~dB}$, which is lower than desired. 

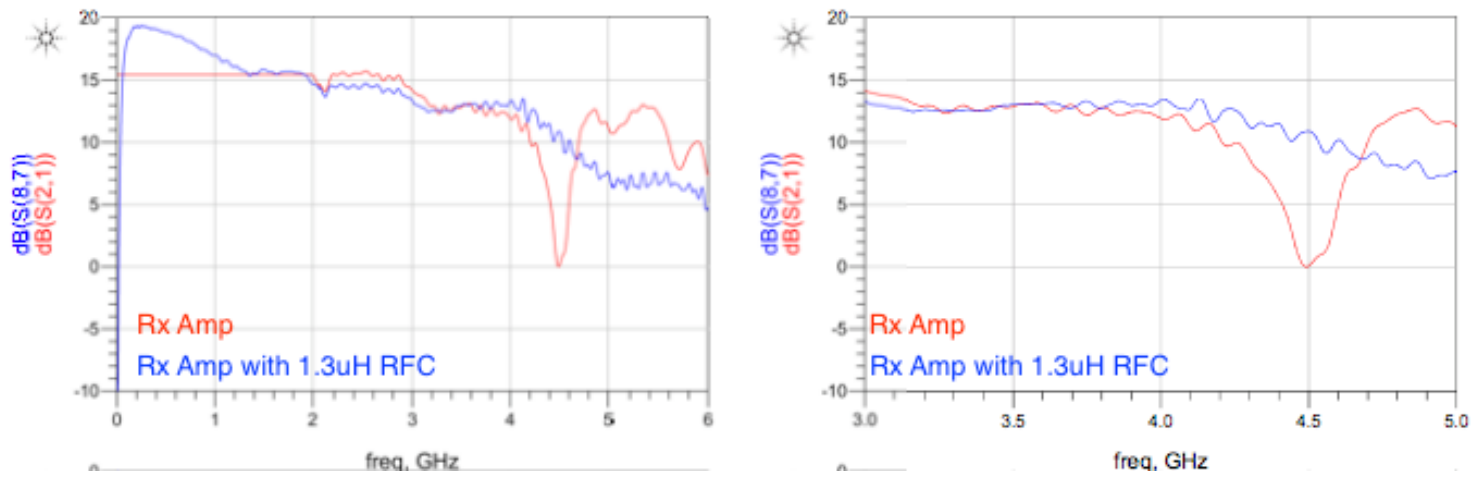

Figure 5.34: Rx Gain Block Amplifier Gain vs 1.3uH RFC

Figure 5.35 shows both the input and output impedance and return loss for the two measurement cases. The output impedance is improved with the $1.3 \mathrm{uH}$ RFC, especially at 4.7 $\mathrm{GHz}$ were it was previously as bad as $2.5 \mathrm{~dB}$.
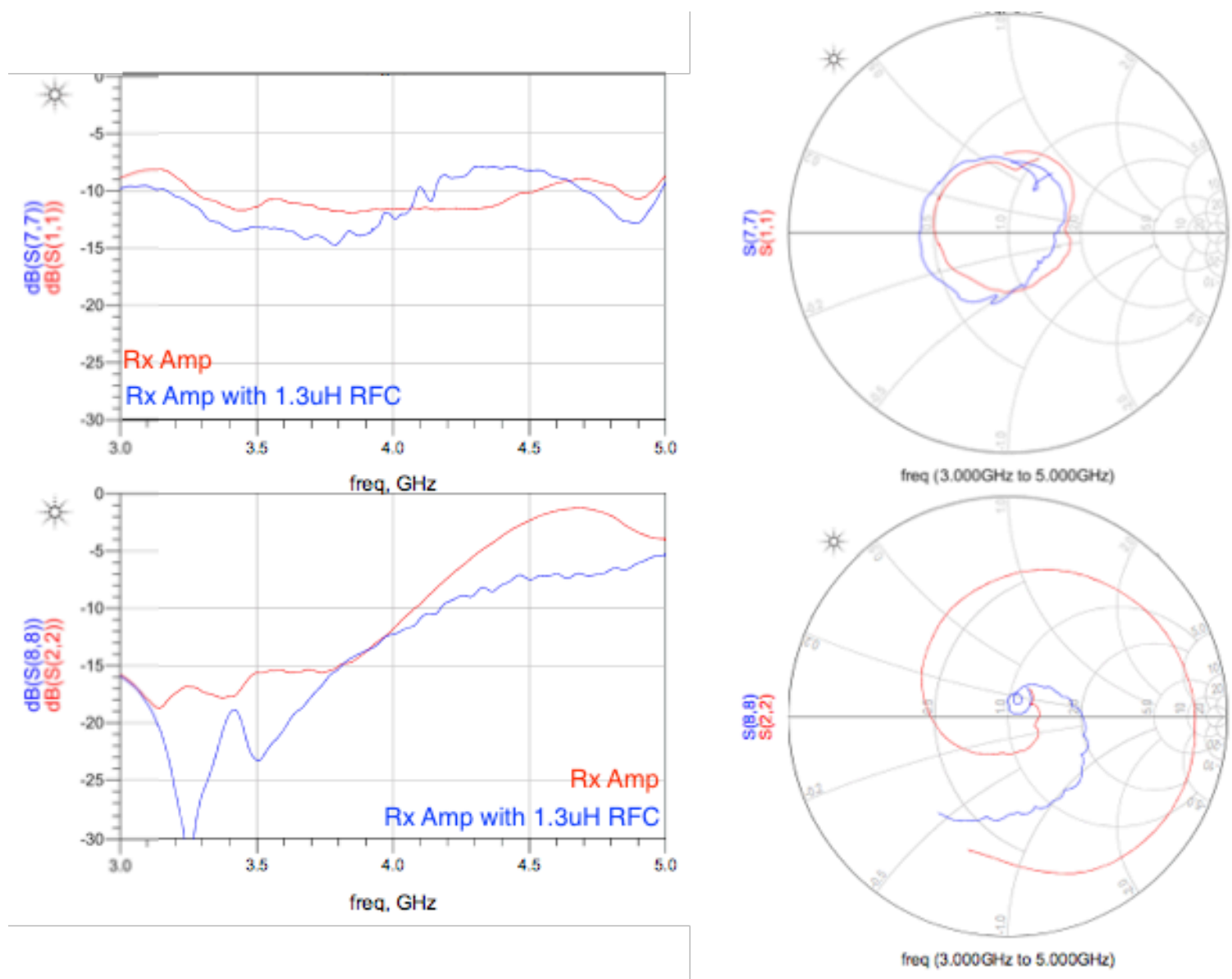

Figure 5.35: Rx Gain Block Amplifier Return Loss and Impedance vs 1.3uH RFC 


\subsubsection{Mixer}

The mixer was characterized by injecting RF signals into the RF and LO ports of the mixer and measuring the output spectrum on the mixer IF port. The IF power was characterized for $\mathrm{LO}$ powers of $17 \mathrm{dBm}$ and $19.4 \mathrm{dBm}$ with an RF signal of $0 \mathrm{dBm}$. Figure 5.36 shows the down converted IF power versus IF frequency for an RF frequency of $3 \mathrm{GHz}$. Similarly, Figure 5.37 shows the down converted IF power versus RF frequency for an IF frequency of $3 \mathrm{MHz}$.

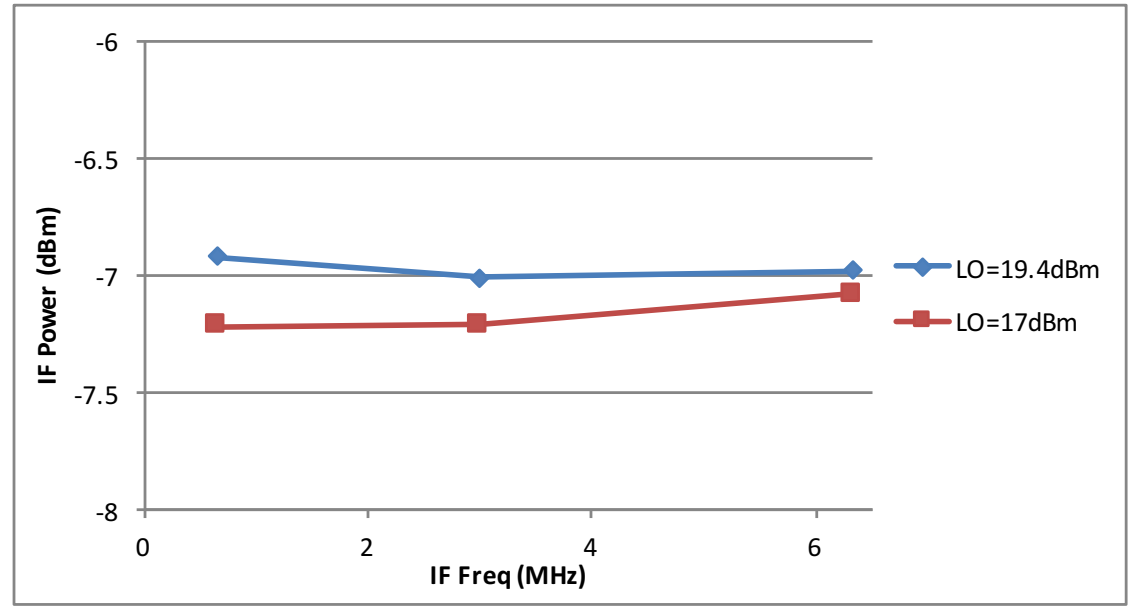

Figure 5.36: Mixer Down Converted Power vs IF Frequency, RF=3GHz

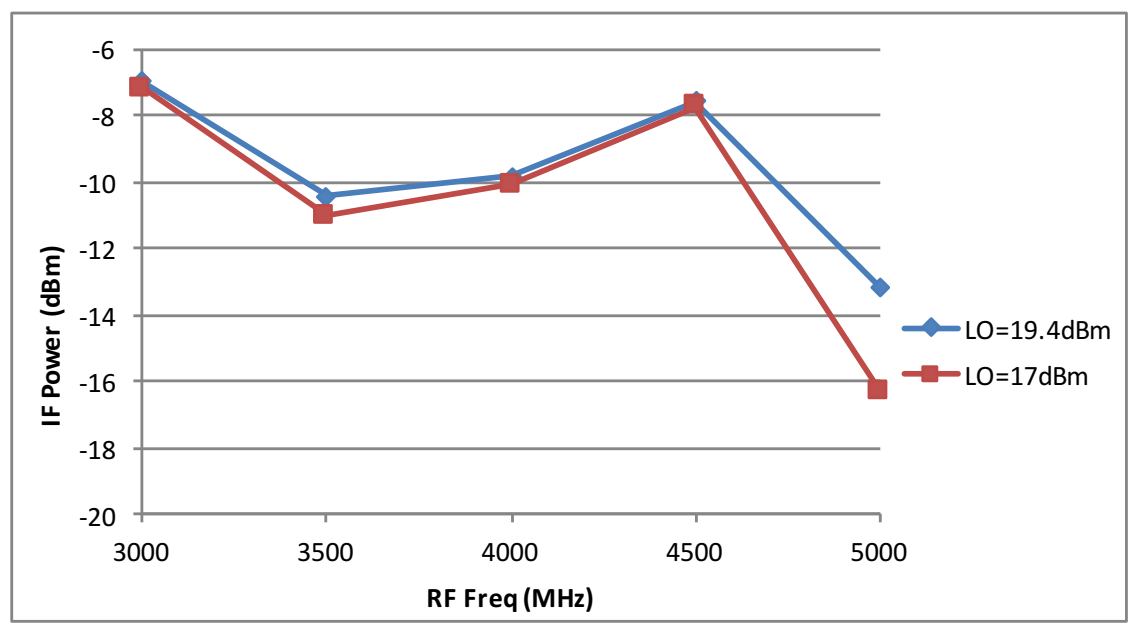

Figure 5.37: Mixer Down Converted Power vs RF Frequency, IF $=3 \mathrm{MHz}$

By comparing the IF powers in Figure 5.36 and Figure 5.37, again we can see the impact of the PCB traces at the higher RF frequencies. The IF power at $5 \mathrm{GHz}$ drops, which is due to 
additional loss and mismatch of the RF signal before reaching the mixer input. The mixer specification for conversion loss is $6 \mathrm{~dB}$ typical, and $9 \mathrm{~dB}$ maximum. This matches the measured data for the lower RF frequencies.

Additionally, the mixer specification for LO to IF isolation is $18-25 \mathrm{~dB}$ typical across frequency. Figure 5.38 below shows a wideband spectrum measurement on the IF output which shows an LO power of $-13 \mathrm{dBm}$, giving a measured LO to IF isolation of $-30 \mathrm{~dB}$ which meets requirements.

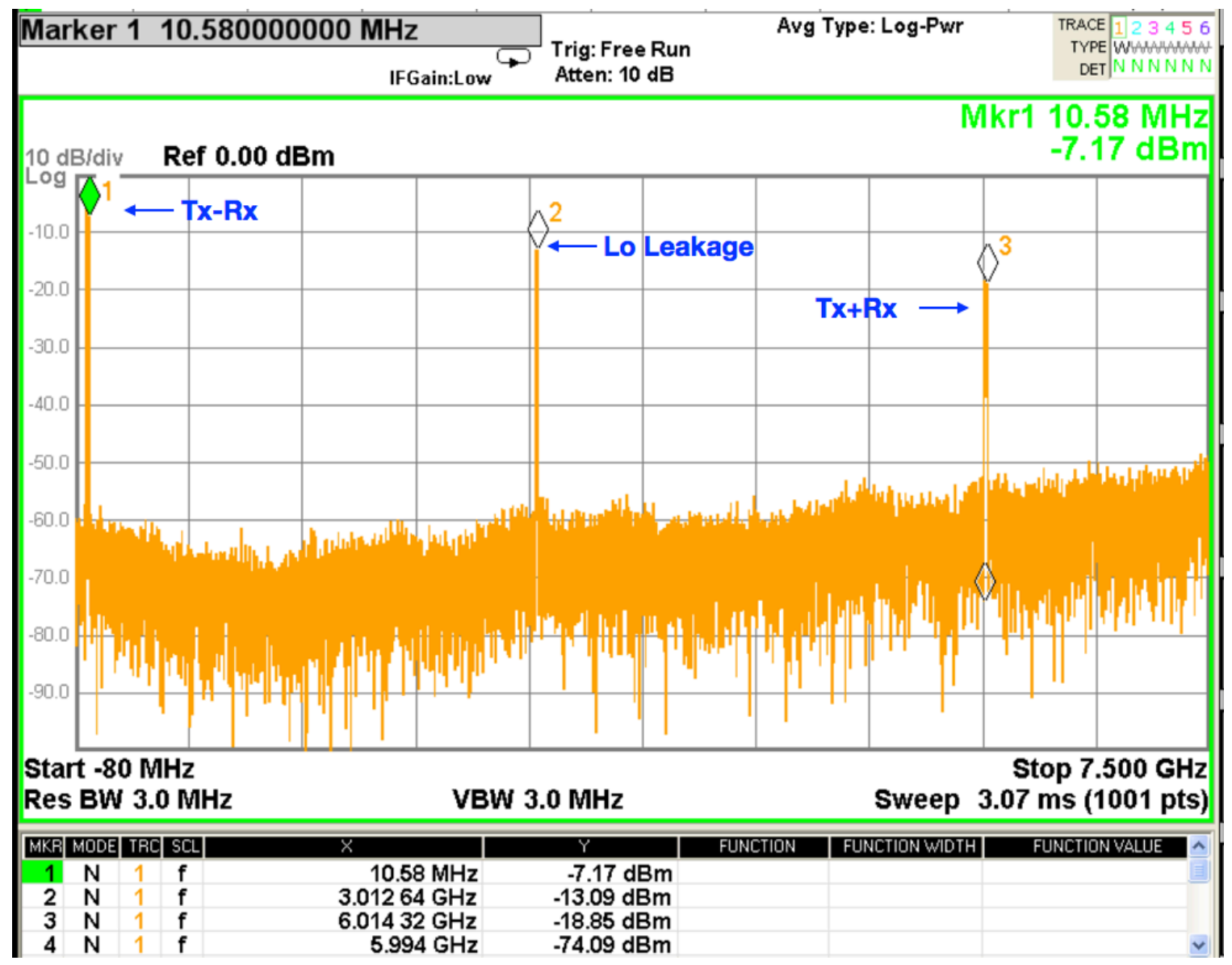

Figure 5.38: Mixer IF Port Leakage and Harmonics

Aside from the desired IF mixing product of RF-LO signals, additional products are generated at $\mathrm{RF}+\mathrm{LO}$ as well as higher order products. The $\mathrm{RF}+\mathrm{LO}$ product is measured at 
$-19 \mathrm{dBm}$, as seen in Figure 5.38, which equates to a conversion loss of $19 \mathrm{~dB}$ for the $0 \mathrm{dBm} \mathrm{RF}$ signal input and is low enough to easily be filtered out.

Higher order even mixing products are shown below in Figure 5.39, and are more than $50 \mathrm{~dB}$ lower than the desired IF signal. These $4^{\text {th }}$ and $6^{\text {th }}$ order mixing products are low enough that the system will not detect them as false objects when they land in the IF passband.

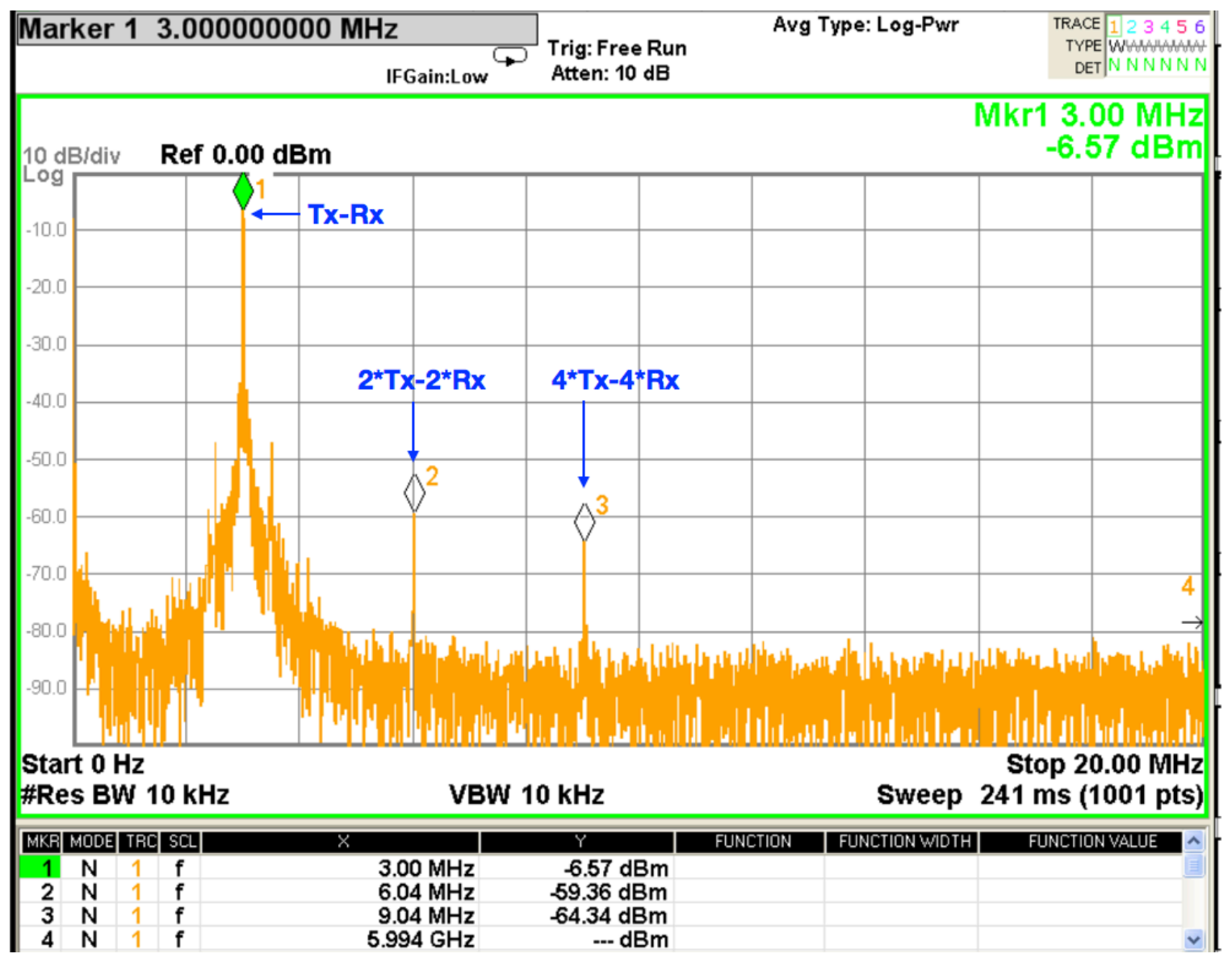

Figure 5.39: IF Frequency and Higher Order Products

\subsubsection{Discrete RC Low Pass Filter}

The RC Low Pass Filter was chosen for rejecting high frequency mixer products. The insertion loss matches the simulated 3.5dB, and is shown in Figure 5.40. 


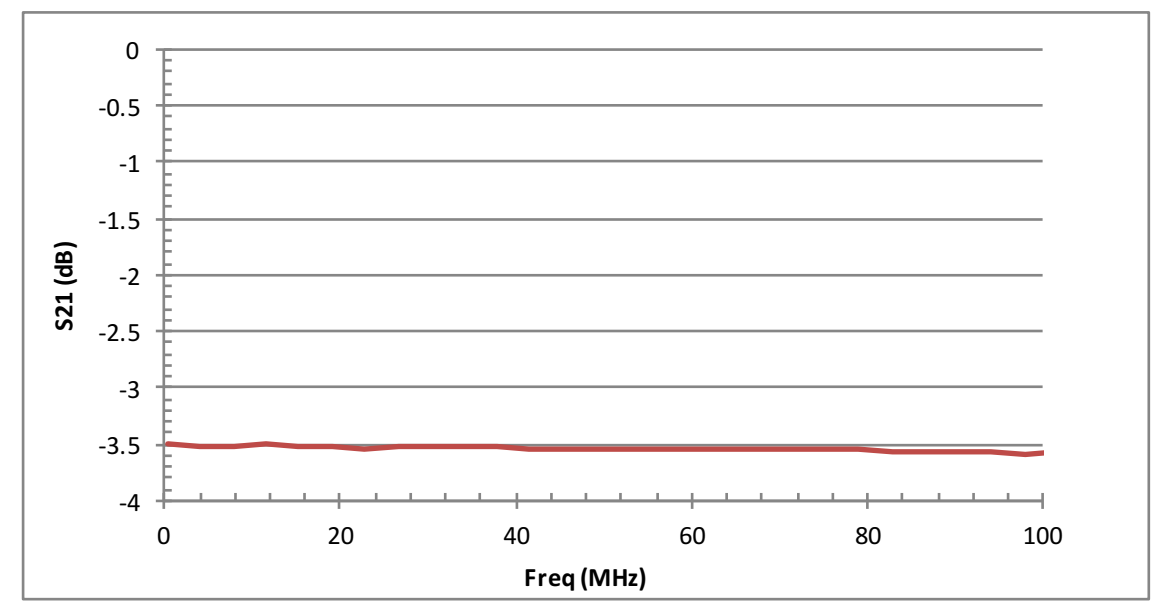

Figure 5.40: RC LPF Insertion Loss

Figure 5.41 shows the out of band attenuation of the RF LPF. The measurement shows that the worst frequencies from $3.1-5 \mathrm{GHz}$ have $10 \mathrm{~dB}$ attenuation, which is $5 \mathrm{~dB}$ worse than the simulated values. The filter notch shifted lower in frequency than the simulation, but remains close enough to provide the desired attenuation.

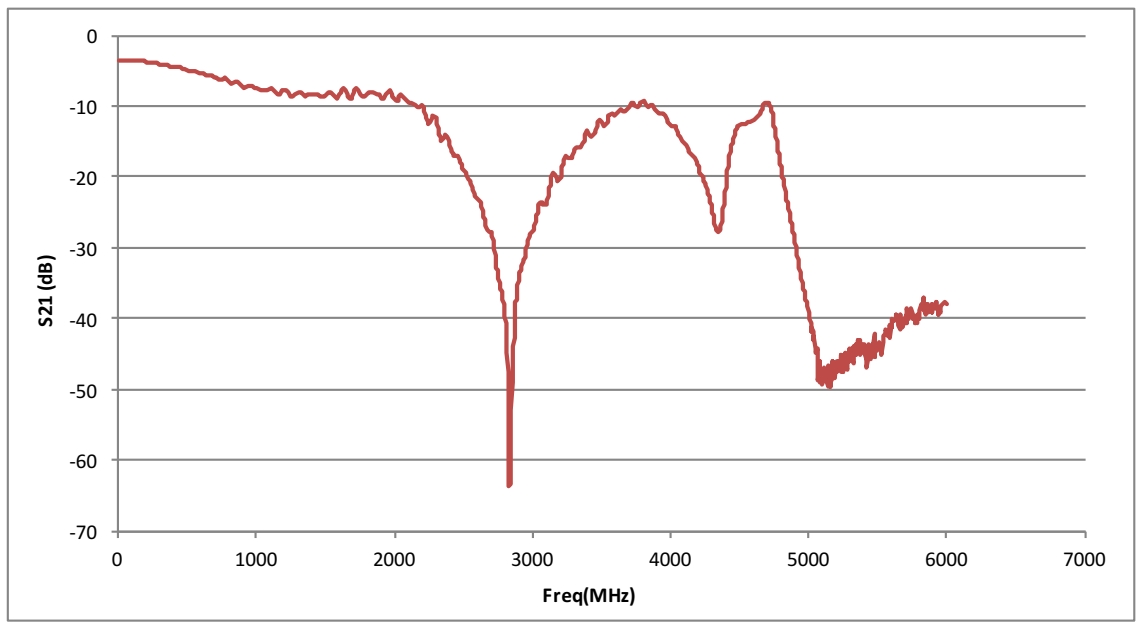

Figure 5.41: RC LPF Attenuation

\subsubsection{Active Low Pass Filter}

The frequency response of the Active Low Pass Filter was measured. Its passband is shown in Figure 5.42, and the wideband attenuation is shown in Figure 5.43. 


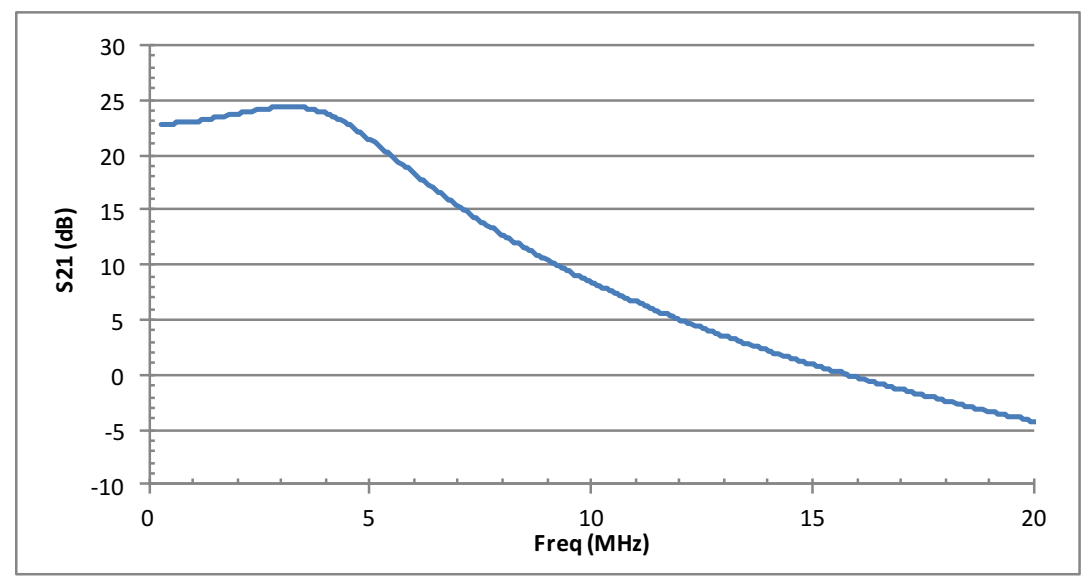

Figure 5.42: Active LPF Passband

The filter passband has a peak gain of $24.37 \mathrm{~dB}$. The $3 \mathrm{~dB}$ cutoff frequency is at $5.035 \mathrm{MHz}$, slightly lower than the target of $6.825 \mathrm{MHz}$. The gain at $6.33 \mathrm{MHz}$, the upper edge of the passband, is $17.22 \mathrm{~dB}$. Overall the in-band filter performance is in line with the simulations and meets required performance.

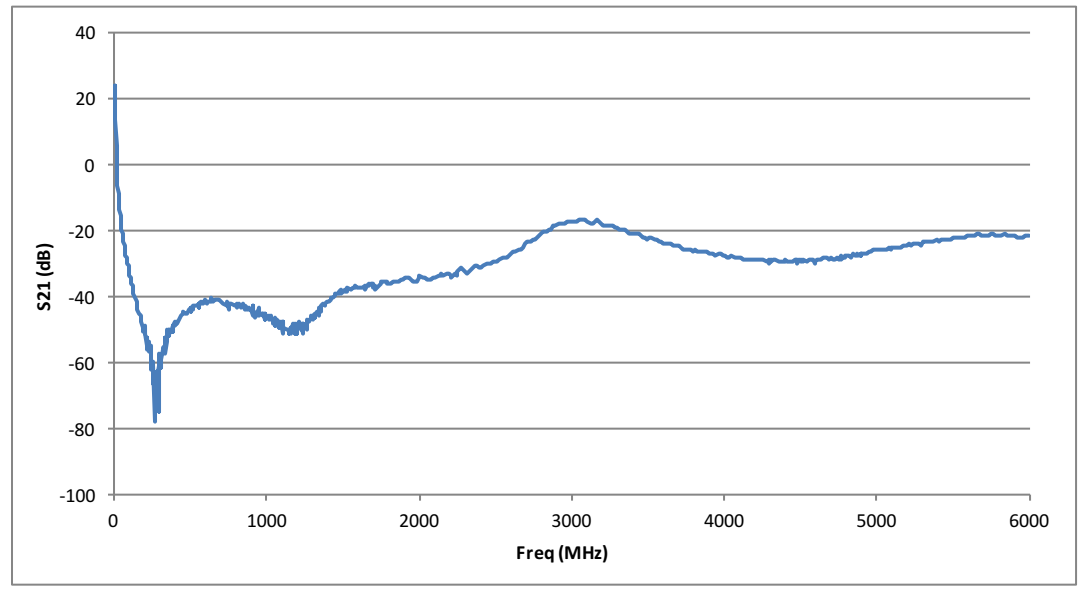

Figure 5.43: Active LPF Attenuation

The out of band attenuation, which was measured up to $6 \mathrm{GHz}$, does not show fly back. The attenuation at $3 \mathrm{GHz}$ peaks at $19 \mathrm{~dB}$, and remains better than $20 \mathrm{~dB}$ up to $6 \mathrm{GHz}$. Out of band attenuation below $2.5 \mathrm{GHz}$ is better than $30 \mathrm{~dB}$. The consistently good attenuation of the Active LPF all the way up to $6 \mathrm{GHz}$ means the RC LPF discussed in Section 5.4.5 is not required for attenuation of the high frequency undesired mixer products. 


\subsubsection{Variable Gain Amplifier}

The variable gain amplifier was characterized with a $1 \mathrm{MHz}$ sin wave input signal, and the output was measured for varying gain settings controlled by $V_{G}$. The input waveform amplitude was scaled between $10 \mathrm{mVp}$ to $100 \mathrm{mVp}$ as $\mathrm{V}_{\mathrm{G}}$ decreased, and amplifier gain decreased correspondingly. Figure 5.44 shows the measured VGA gain across $\mathrm{V}_{\mathrm{G}}$.

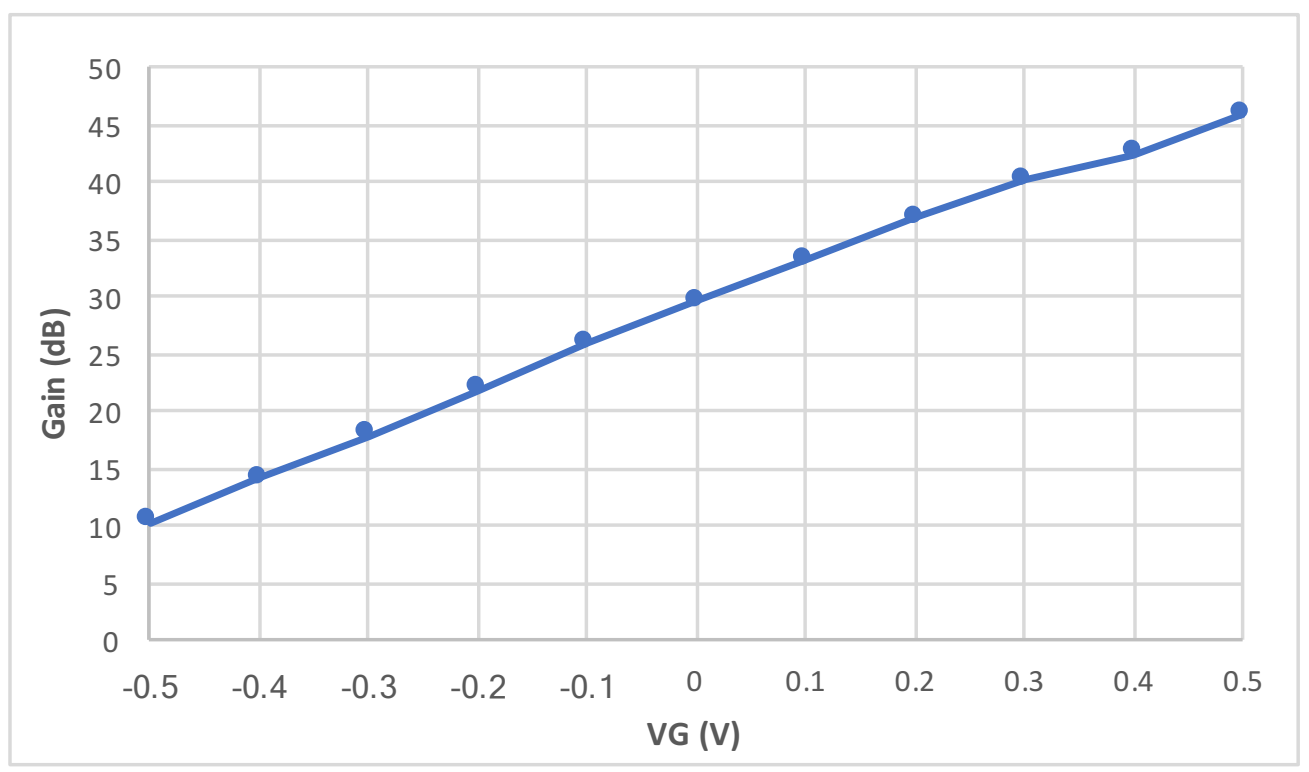

Figure 5.44: VGA Gain

The required minimum VGA gain is $10.05 \mathrm{~dB}$, and required maximum gain is $39.90 \mathrm{~dB}$. This corresponds to an output voltage between 200mVpp and 3.3Vpp across all VGA input levels, between $2.023 \mathrm{mVpp}$ and $1.037 \mathrm{Vpp}$. The VGA characterization shows its performance meets the system requirements.

\subsection{Component Discussion}

Section 5.3 and 5.4 presented measured data for each component in the transmitter and receiver system chains respectively. Multiple components did not meet their performance targets, those components are summarized here and root cause for the issues are discussed. 


\subsubsection{High Frequency Amplifiers}

The major group of components that weren't meeting their design specifications were the high frequency amplifiers. This group includes the Tx Gain Block, Tx Power Amplifier, LNA, and Rx Gain Block. Section 5.3.8 discusses the Tx Power Amplifier, showing that its initial measured performance had a frequency resonance causing a gain suck out, as well as low gain and return loss at higher frequencies.

The frequency resonance was solved by adding a $1.3 \mathrm{uH}$ wideband bias RF choke between the power supply and amplifier. This adds isolation at RF frequencies and prevents the RF from shunting to the power supply rail, causing losses and reduced gain at RF frequencies.

As discussed in Section 5.2, the PCB traces have high loss and poor impedance above 3GHz. The Tx Power Amplifier measurements in Section 5.3.8 show the impact of this. The initial measurements were taken at the zero ohm resistors separating the input and output of the amplifier from the next component in the chain. By moving that reference plane to the input and output matching components, the trace length in the measurement is reduced by $394 \mathrm{mil}$ and $681 \mathrm{mil}$ respectively. This increased the measured gain by $5.5 \mathrm{~dB}$ at $5 \mathrm{GHz}$ and improved the return loss by an average of $7 \mathrm{~dB}$ across the band.

An additional negative impact that the PCB can have on amplifier performance is due to the PCB's via inductance to ground. The thick PCB, at 0.062in, means the via length from the lop layer component ground pin down to the bottom layer ground return plane is fairly long. If an insufficient number of vias are placed at the component ground pin in parallel, then the via series inductance at high frequencies like $3-5 \mathrm{GHz}$ is significant. An example for how series inductance to ground can degrade amplifier performance is emitter degeneration in the common emitter amplifier configuration, where placing an inductor or resistor between the emitter and ground reduces the amplifier gain [59]. 


\subsubsection{Passive Components}

Multiple passive components did not meet target performance specifications, including the Microstrip Stepped Impedance LPF, Power Splitter, and Output Power Attenuator. As discussed in Section 5.3.9, the power splitter was not successfully soldered to the PCB. Either a more precise soldering method or a different power splitter package with larger pin sizes could help solve this issue.

Section 5.3.6 discusses how the Microstrip Stepped Impedance LPF performance did not match the initial piecewise schematic level simulation. A higher order 3D EM model simulation using ADS Momentum was presented which predicted the measured performance. The $3-5 \mathrm{GHz}$ operating range causes the width of the filter arms to be significantly larger than the length, causing coupling between the arms which changes the filter frequency response. Further review of the transmitter level plan at the third harmonic output levels compared to the system requirement of $-51.3 \mathrm{dBm}$ output power above $10.6 \mathrm{GHz}$ shows that the original system design has more than $50 \mathrm{~dB}$ margin to this requirement. The third harmonic attenuation provided by the LPF is not required for the system, and this component can be removed in future designs.

The Output Power Attenuator design requires $41.5 \mathrm{~dB}$ of attenuation. Achieving such high attenuation requires careful consideration to ensure that the attenuator response is not dominated by insufficient isolation across the components. The chosen design only met $18 \mathrm{~dB}$ of attenuation at high frequencies, which was dominated by the isolation across the single series resistor in the PI network. Section 5.3.11 discusses how dividing this block into multiple cascaded lower value attenuators could solve the isolation issue. Alternatively, the system architecture could be redone in future designs to prevent needing such a large attenuation at the system output. 


\subsubsection{Bandwidth Limited Components}

Some components met performance targets over most of the operating band, but show significant roll off at the upper band edge. These components include the Low Frequency Tx Amplifier, the Coupler, and the Mixer.

The Coupler and Mixer have degraded insertion loss at $5 \mathrm{GHz}$, which is caused by the PCB traces that are included in the measurement. The traces have high insertion loss, as well as a non-50 $\Omega$ impedance which further degrades the component performance- as the components are designed to operate with $50 \Omega$ termination impedances.

The Low Frequency Tx Amplifier operates from 1.5-2.5GHz, which means it is not impacted by the poor PCB traces like the high frequency components. Instead, the Low Frequency Tx Amplifier is limited by the wide bandwidth requirements. As discussed in Section 5.3.3, the $1 \mathrm{GHz}$ bandwidth and 50\% fractional bandwidth requirements mean the amplifier matching cannot be optimized. The amplifier has good gain at $1.5 \mathrm{GHz}$ where the input impedance is $11 \mathrm{~dB}$, but at $2.5 \mathrm{GHz}$ the wide impedance contour cannot be matched to $50 \Omega$ without compromising the low frequency impedance. At $2.5 \mathrm{GHz}$ the gain drops by $5 \mathrm{~dB}$ as the input return loss reaches $3.5 \mathrm{~dB}$. 


\section{Conclusions and Future Work}

This paper discusses the current systems used for eldercare monitoring, including cameras, wearables, and household sensors. Limitations of these systems, most notably line of sight, user privacy, and invasiveness, leave a need for new solutions. A monitoring system based on a frequency modulated continuous wave ultra-wideband short-range radar is proposed for this application. A custom design is pursued for the radar system which allows hardware optimization for the specific application in order to reduce power consumption, cost, and complexity. Furthermore, a custom design allows future expansion for additional features such as human identification. The complete proposed monitoring system is comprised of four blocks: boundary detection, silhouette capture, human identification, and data transmission.

System requirements are derived for the silhouette capture subsystem. An architecture for the RF front end is designed, and required individual component specifications are determined. Components are selected off the shelf or custom designed for each socket. Full transmitter and receiver level plans are calculated to ensure expected system performance meets system requirements for transmit power, LO power, third harmonic, maximum and minimum detectable signals, and receiver third order intermodulation products. A DipTrace component library is created, including schematic symbols and land pattern footprints. A full system schematic is created, PCB layout is completed, and PCB gerber files are generated and sent for fabrication. Components are soldered to the PCB by manually applying solder paste to each pad, mounting on the PCB, and using a solder reflow oven. PCB traces and individual components are characterized over frequency, and methods that improve inadequate performance are documented and discussed.

The PCB RF traces show good performance up to $3 \mathrm{GHz}$. Above that, they shift away from the target $50 \Omega$ characteristic impedance and have increased insertion loss. At $4.5 \mathrm{GHz}$ an inband resonance is seen with a corresponding drop in insertion loss. The high frequency 
amplifiers have similar resonances which decrease gain. These are mitigated by adding a wideband RFC on the supply line, however the poor PCB trace performance at the high operating frequencies also contributes to low gain across the band. The microstrip stepped impedance low pass filter also does not perform as intended, but the system performance at harmonic frequencies due to the BPF out of band attenuation means the stepped impedance low pass filter can be removed from the front-end architecture.

Overall this paper advances work on an ongoing eldercare monitoring system project to extend independent living. The system requirements for the silhouette capture frequency modulated continuous wave ultra-wideband RF front-end are clearly derived. An initial system architecture is defined and implemented- from component specification derivation, to system level plan performance calculations, through schematic and PCB layout. Each component of the RF front-end is fully characterized, problems are analyzed, and fixes are proposed or implemented. The following sections discuss possible future improvements for the silhouette capture RF front-end based on learnings from this project, as well as remaining future projects to complete the entire silhouette capture subsystem.

\subsection{Silhouette Capture RF Front-End Future Improvements}

Improvements can be made on the RF front-end architecture defined in this paper. A major constraint placed on the hardware during the design phase was keeping a symmetrical group delay between the two LO paths, and a minimal group delay variation between the RF path and LO paths. This was done with the intent of minimizing the error in the down converted IF signal which would correspond to errors in the detected objects distances. Rather than constraining the hardware with this requirement, the final hardware system can be calibrated and a software correction can compensate for these group delay deltas to provide an accurate detected object distance. By removing this hardware requirement, a few improvements can be made to the 
architecture. Most notably the transmitter amplifiers, which are required to increase the signal to drive the mixer LO ports, can be moved to after the RF transmit and LO split. This also means that the large output attenuator is no longer required on the transmit signal. Similarly, the second coupler placed on the other LO path can be removed to save cost, insertion loss, and board area.

An additional architecture simplification that can be made is to remove unnecessary filters that were placed for additional harmonic attenuation. The microstrip stepped impedance low pass filter on the transmit chain can be removed, as the bandpass filter provides enough attenuation to meet the system performance requirements. Similarly, the passive RC low pass filter on the receiver chain was included to compensate for attenuation fly back in the active low pass filter at higher frequencies. Characterization of the active low pass filter across frequency shows good attenuation up to $6 \mathrm{GHz}$, which means the passive $\mathrm{RC}$ filter can be removed.

Finally, the FMCW waveform is generated using a VCO which operates at half the fundamental frequency and is then doubled by a frequency multiplier. This was chosen because off the shelf VCOs capable of outputting $3-5 \mathrm{GHz}$ were outside of the project budget. The frequency multiplier adds nonlinearities which are undesired and require additional filtering. Secondly, doubling the VCO output frequency also results in doubling the phase noise on the signal. This is undesired as it reduces the precision in the down converted resolution, which can manifest as a small error in the object's distance. Finding a higher frequency VCO which allows removal of the frequency multiplier would avoid these undesired effects.

Aside from improvements to the front-end system architecture, improvements to the PCB layout are also needed. The implemented RF traces do not have a $50 \Omega$ characteristic impedance up to $5 \mathrm{GHz}$, and have poor insertion loss. As discussed in Section 5.2, increasing trace width from 121 mil to $158 \mathrm{mil}$ could give the traces a $50 \Omega$ characteristic impedance from $3-5 \mathrm{GHz}$, with the tradeoff of worse performance at lower frequencies where they aren't intended to operate. Other methods of improving the trace characteristic impedance and insertion loss at high 
frequencies were not pursued for this project due to high cost. If desired, the PCB fab could use impedance controlled traces, where they dynamically adjust the trace width to achieve the target characteristic impedance. Additionally, higher quality materials can operate better at high frequencies with lower insertion loss compared to the cheaper FR4 used for this project. Finally, the layout can also be improved by moving to a shorter board stack up. This would reduce the height of the dielectric between the RF traces and ground, and result in a narrower $50 \Omega$ trace width. Thinner traces would cause less of an impedance discontinuity between the component pins and the trace impedance, and would also allow for a more compact PCB layout with shorter trace lengths between components. Finally, a shorter board stack up would also mean shorter via lengths which would reduce the inductance to ground.

\subsection{Remaining Silhouette Capture Subsystem Projects}

This paper focused on the RF front-end portion of the silhouette capture subsystem. A complete silhouette capture subsystem requires the following additional sections. Most obvious is an antenna designed to radiate at the system operating frequencies of 3.1-4.9GHz. Also not included in this paper is the design for the sawtooth wave generator, which outputs $0-10 \mathrm{~V}$ at $100 \mathrm{kHz}$ before being doubled with a non-inverting op amp. The remaining next steps are digitally sampling the down converted IF spectrum and processing the results. The software backend could include smart algorithms to ignore the IF pulses at the end of the sawtooth waveform period when the transmit frequency rolls over, or it could compensate for this rollover to continue to get valid detection data during this time. Additionally, it could look into defining FFT windows for segmented scans of a room to mitigate processing complexity due to multipath reflections. Different scan types could be implemented to optimize for accurate object detection which requires peak IF frequency power detection, compared to object identification which requires power versus frequency information and how it changes over time. A final goal for the 
system is to expand from human detection into the identification of specific humans. This requires specification derivation and corresponding changes in the system design, similar to what was presented in this paper for human detection. 


\section{REFERENCES}

[1] A. a. T. Mack, "A Passive and Portable System for Monitoring heart Rate and Detecting Sleep Apnea and Arousals: Preliminary Validation," in Proceedings of the Transdisciplinary Conference on Distributed Diagnosis and Home Healthcare, 2006.

[2] G. A. M. Aud, "Use of Sensor System Data for Early Detection of Health Status Changes in Older Adult Residents of a Retirement Community," Midwest Nursing Research Society, Mar 2007.

[3] M. S. M. Rantz, "Using Technology to Enhance Aging in Place," in Intl Conference on Smart Home and Health Telematics, Ames, IA, 2008.

[4] L. a. S. Anderson, "Evaluation of a Video Based Fall Recognition System for Elders Using Voxel Space," in Intl Conference of the Intl Society for Gerontechnology, Pisa, Tuscant, Italy, 2008.

[5] R. a. K. Alwan, "A Smart and Passive Floor-Vibration Based Fall Detector for Elderly," in Proceedings of the 2nd IEEE Intl Conference on Information and Communication Technologies: From Theory to Applications, Damascus, Syria, 2006.

[6] A. Yarovoy, J. Matuzas, B. Levitas and L. P. Ligthart, "UWB Radar for Human Being Detection," IEEE Radar Confrence, p. 3, 2005.

[7] C. Wolff, "Free-space Path Loss," [Online]. Available: http://www.radartutorial.eu/18.explanations/ex44.en.html. [Accessed 24 April 2013].

[8] D. M. Pozar, Microwave and RF Wireless Systems, New York: John Wiley \& Sons, Inc., 2001.

[9] Antenna-Theory, "The Friis Equation," 2011. [Online]. Available: http://www.antennatheory.com/basics/friis.php. [Accessed 24 April 2013].

[10] G. Shingu, K. Takizawa and T. Ikegami, "Human Body Detection Using UWB Radar in an Indoor Environment," IEEE Communications and Infomration Technolgoies, p. 4, 2008.

[11] J. A. Adam, "How to Design an Inivisible Aircraft," IEEE Spectrum, p. 28, 1988.

[12] C. N. Paulson, J. T. Chang, C. E. Romero, J. Watson, F. Pearce and N. Levin, "Ultrawideband Radar Methods and Techniques of Medical Sensing and Imaging," in SPIE International Symposium on Optics East, Boston, 2005.

[13] M. Di Renzo, R. Buehrer and T. J., "Pulse Shape Distortion and Ranging Accuracy in UWBBased Body Area Networks for Full-Body Motion Capture and Gait Analysis," IEEE Global Telecommunications Confrence, p. 2, 2007.

[14] R. Aiello, Ultra Wideband Systems, Burlington: Elsevier, 2006.

[15] Z. Sahinoglu, Ultra-wideband Positioning Systems, Cambridge, New York: Cambridge University Press, 2008.

[16] "Subpart F - Ultra-Wideband Operation," Federal Communications Commission, pp. 839, 845 .

[17] B. Allen, Ultra-wideband Antennas and Propagation for Communications, Radar and 
Imaging, West Sussex, England: John Wiley \& Sons Ltd, 2007.

[18] W. Tang, “A Frequency-Modulated Continuouswave-Based Boundary Detection System for Determination of Monitoring Region for an Indoor Ultra-Wideband Short Range RadarBased Eldercare Monitoring System,” California Polytechnic State University, 2010.

[19] O. a. K. MacGougan, "Ultra-wideband Ranging Precision and Accuracy," Measurement Science and Technology, 2009.

[20] T. W. Barrett, "History of Ultra Wideband Communications and Radar," UWB Communications, pp. 22-56, 2001.

[21] F. G. Ross, "Transmission and Reception System for Generating and Receiving Base-Band Duration Pulse Signals without Distortion for Short Base-Band Pulse Communication System". US Patent 005519400, 1973.

[22] T. E. McEwan, "Phase Coded, Micro-Power Impulse Radar Motion Sensor". US Patent 3728632, 1995.

[23] M. a. P. Maaref, "A Study of UWB FM-CW Radar for the Detection of Human Beings in Motion Inside a Building," IEEE Transactions on Geoscience nad Remote Sensing, 2009.

[24] T. Elmissaoui, N. Soudani and R. Bouallegue, "The Reflection of Electromagnetic Field by Body Tissue in the UWB Frequency Range," IEEE Radar Confrence, 2010.

[25] D. Andreuccetti, R. Fossi and C. Petrucci, "Dielectric Properties of Body Tissues," Institute for Applied Physics, 2012. [Online]. Available: http://niremf.ifac.cnr.it/tissprop/htmlclie/htmlclie.htm. [Accessed 4 May 2012].

[26] C. Gabriel, G. S. and C. E., "The Dielectric Properties of Biological Tissues: I. Literature Survey," Physics in Medicine and Biology, 1996.

[27] S. Gabriel, R. W. Lau and C. Gabriel, "The dielectric properties of biological tissues: II. Measurements in the frequency range $10 \mathrm{~Hz}$ to $20 \mathrm{GHz}$," Physics in Medicine and Biology, 1996.

[28] S. Gabriel, R. W. Lau and C. Gabriel, "The dielectric properties of biological tissues: III. Parametric models for the dielectric spectrum of tissues," Physics in Medicine and Biology, 1996.

[29] S. Gunishetty, "Uniquely Identifying a Human Amoung a Clutter using Ultrawideband Short Range Radar," University of Missouri-Colombia, 2010.

[30] A. Nezirovic, M. Liu and A. Yarovoy, "Modelling of Reflection of UWB Pulses from Trapped Human Beings," Ultra-Wideband, Short-Pulse Electromagnetics.

[31] A. Nezirovic, A. Yarovoy and L. Ligthart, "Experimental verification of Human Being Detection Dependency on Operational UWB Frequency Band," Ultra-Wideband, IEEE International Conference, 2007.

[32] [Online]. Available: http://www.ixbt.com/comm/images/uwb/uwb2.png. [Accessed 20 April 2013].

[33] A. Yarovoy, X. Zhuge, T. Savelyev and L. Ligthart, "Comparison of UWB Technologies for Human Being Deteciton with Radar," International Research Centre for 
Telecommunications-transmission and Radar, 2007.

[34] [Online]. Available: http://dmtradar.com/wp-content/uploads/2012/07/FMCWWaveform.png. [Accessed 30 April 2013].

[35] A. Patel, "Signal Generation for FMCW Ultra-Wideband Radar," University of Kansas, 2009.

[36] [Online]. Available: http://www.altera.com/technology/systemdesign/images/Cutting_Through_fig2.jpg. [Accessed 11 Feb 2013].

[37] [Online]. Available: http://en.wikipedia.org/wiki/Operational_amplifier_applications\#/media/File:Op-Amp_NonInverting_Amplifier.svg. [Accessed 25 April 2015].

[38] S. Franco, Design with Operational Amplifiers and Analog Integrated Circuits, 3rd Edition ed., McGraw-Hill, 2001.

[39] Texas Instruments, "LM6211 Low Noise, RRO Operational Amplifier with CMOS Input and 24V Operation," March 2013. [Online]. Available: http://www.ti.com/lit/ds/snosah2c/snosah2c.pdf.

[40] Mini-Circuits, "Voltage Controlled Oscillator ROS-2700-1819+," [Online]. Available: http://www.minicircuits.com/pdfs/ROS-2700-1819+.pdf.

[41] Skyworks, October 2014. [Online]. Available: http://www.skyworksinc.com/uploads/documents/SKY67014_396LF_201515D.pdf.

[42] Mini Circuits, "x2 Frequency Multiplier KSX2-722+," [Online]. Available: http://www.minicircuits.com/pdfs/KSX2-722+.pdf.

[43] Digikey, TDK, August 2006. [Online]. Available: http://media.digikey.com/pdf/Data\%20Sheets/TDK\%20PDFs/DEA453960BT-3002B1.pdf.

[44] Analog Devices, 2007. [Online]. Available: http://www.analog.com/media/en/technicaldocumentation/data-sheets/ADL5541.pdf.

[45] Avago Technologies, February 2011. [Online]. Available: http://www.avagotech.com/docs/AV02-1986EN.

[46] Mini Circuits, "Power Splitter/Combiner GP2X+," [Online]. Available: http://www.minicircuits.com/pdfs/GP2X+.pdf.

[47] Mini Circuits, "High Power Bi-Directional Coupler SYBD-16-53HP+," [Online]. Available: http://www.minicircuits.com/pdfs/SYBD-16-53HP+.pdf.

[48] Avago Technologies, "MGA-71543 Low Noise Amplifier with Mitigated Bypass Switch," January 2006. [Online]. Available: http://www.avagotech.com/docs/AV02-3597EN.

[49] Analog Devices, "Analog Devices ADL5542 50MHz to 6GHz RF/IF Gain Block," October 2007. [Online]. Available: http://www.analog.com/media/en/technical-documentation/datasheets/ADL5542.pdf.

[50] Mini Circuits, "Mini Circuits SIM-762H+ Frequency Mixer Wide Band Level 17," [Online]. Available: http://www.minicircuits.com/pdfs/SIM-762H+.pdf. 
[51] Murata, "Library for Keysight ADS," 18 July 2014. [Online]. Available: http://www.murata.com/en-us/tool/library/keysight2.

[52] Texas Instruments, "Texas Instruments LMH6639 190MHz Rail to Rail Output Amplifier with Disable," March 2013. [Online]. Available: http://www.ti.com/lit/ds/symlink/lmh6639.pdf.

[53] Analog Devices, "Analog Devices AD603 Low Noise, 90 MHz Variable Gain Amplifier," April 2012. [Online]. Available: http://www.analog.com/media/en/technicaldocumentation/data-sheets/AD603.pdf.

[54] Micrel, "Micrel MIC5335 Dual, High Performance 300mA uCap ULDO," May 2008. [Online]. Available: http://www.micrel.com/_PDF/mic5335.pdf.

[55] [Online]. Available: http://images.cnblogs.com/cnblogs_com/yapzhang/201108/201108101723458497.png . [Accessed 25 July 2015].

[56] Isola, "Isola FR402," [Online]. Available: http://www.isola-group.com/products/FR402/. [Accessed 5 March 2016].

[57] R. Hartley, "Jefferson Lab," L-3 Avionics Systems, [Online]. Available: https://www.jlab.org/accel/eecad/pdf/050rfdesign.pdf. [Accessed May 2016].

[58] Keysight Technologies, "Momentum 3D Planar EM Simulator," [Online]. Available: http://www.keysight.com/en/pc-1887116/momentum-3d-planar-emsimulator?cc=US\&lc=eng. [Accessed May 2016].

[59] K. Whites, "The South Dakota School of Mines and Technology Department of Electrical and Computer Engineering," June 2016. [Online]. Available: http://whites.sdsmt.edu/classes/ee320/notes/320Lecture19.pdf. [Accessed July 2017].

[60] S. Winder, Filter Design, Woburn: Newnes, 1997.

[61] [Online]. Available: http://dmtradar.com/wp-content/uploads/2012/07/FMCWWaveform.png. [Accessed April 2014]. 
APPENDICES

APPENDIX A: System Schematic

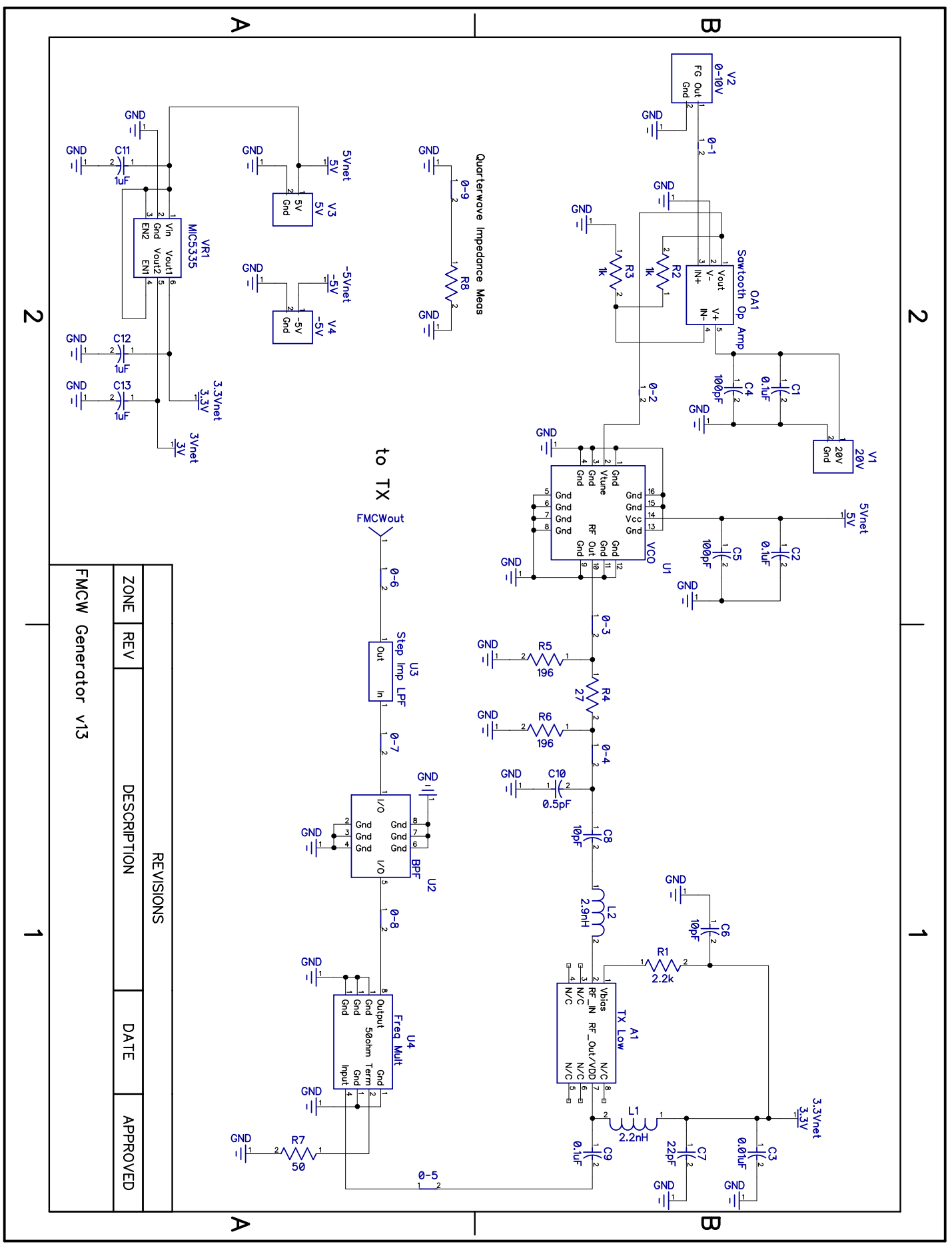




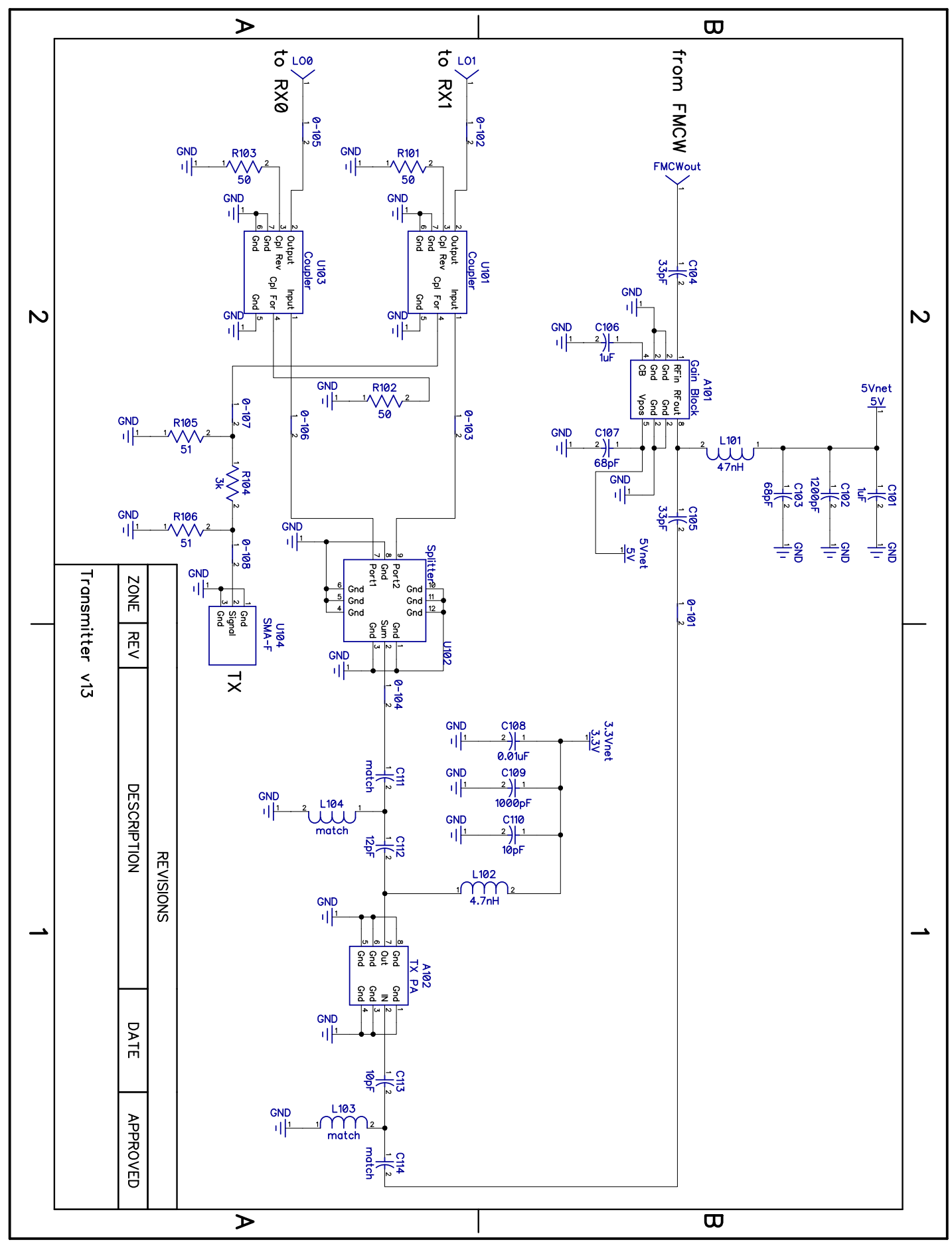




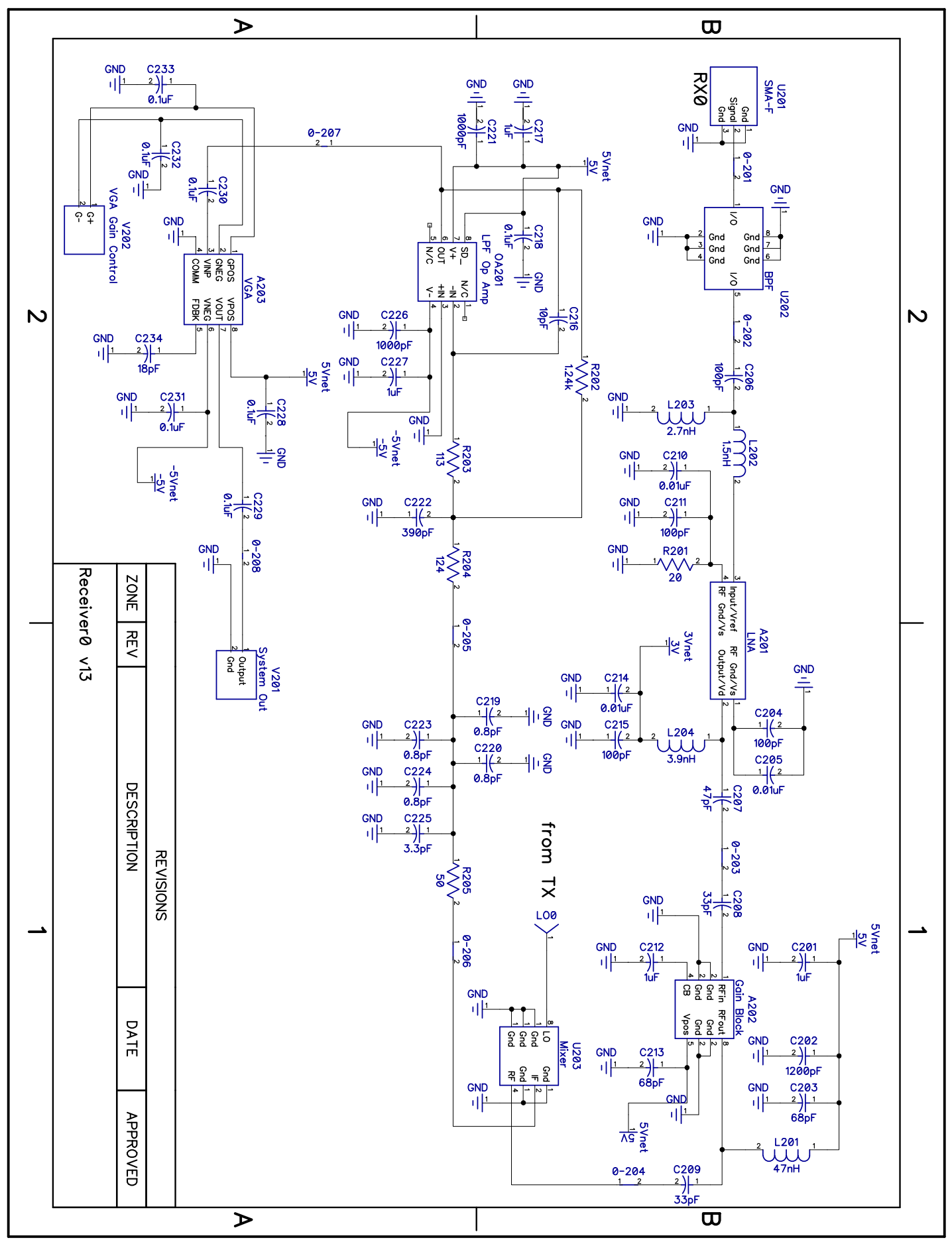




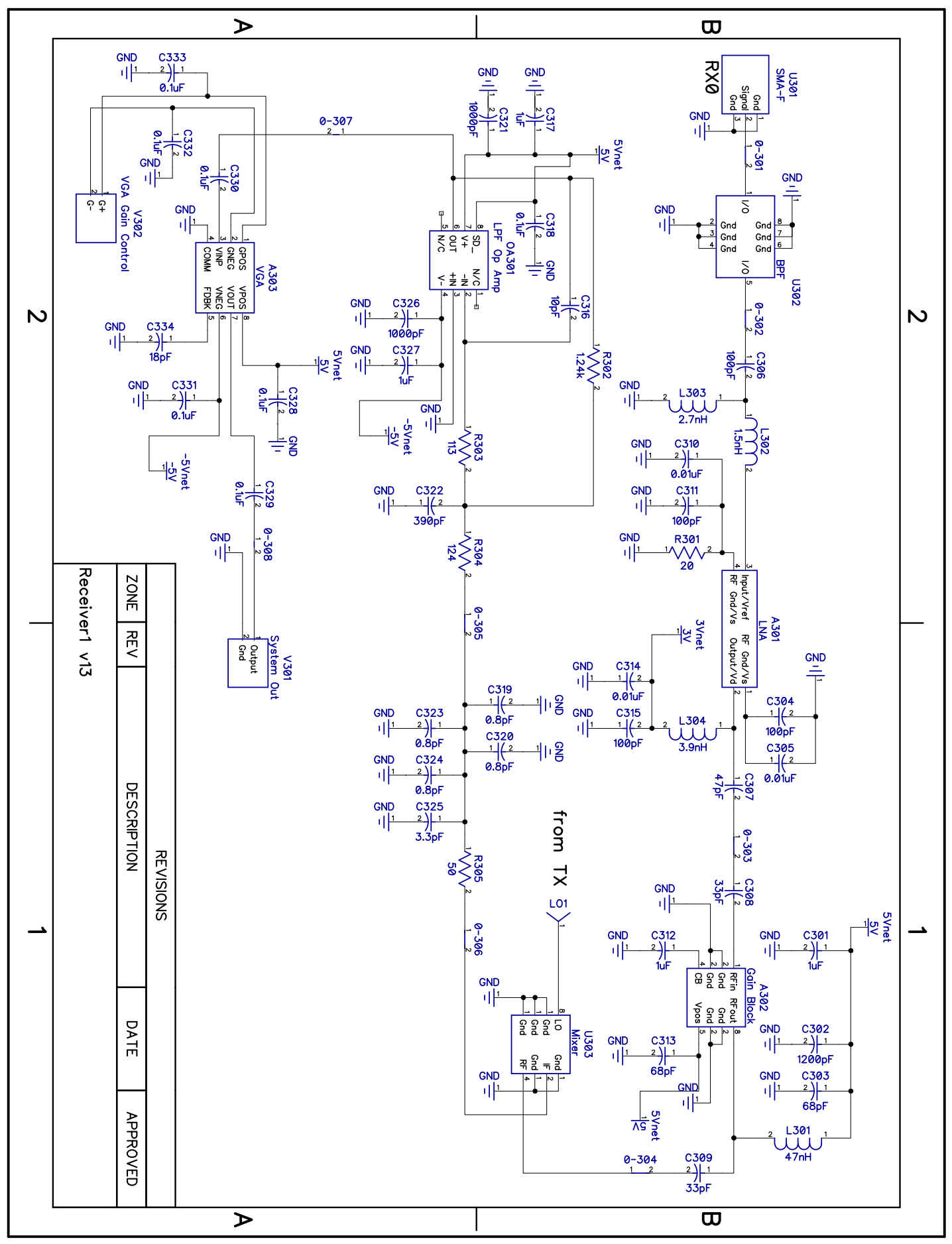


APPENDIX B: Bill of Materials

\begin{tabular}{|c|c|c|c|c|c|c|c|}
\hline & Part & Manufacturer & Distributor & Model Number & Package & Unit Price (\$) & Qty/Board \\
\hline \multirow[b]{15}{*}{ ICs } & FG Op Amp & $\mathrm{TI}$ & Digikey & LM6211 & 5 pin SOT-23 & $\$ 2.96$ & 1 \\
\hline & VCO & MiniCircuits & MiniCircuits & ROS-2700-1819+ & - & $\$ 29.95$ & 1 \\
\hline & TX Amp Low & Skyworks & Digikey & SKY67014-396LF & 8pin DFN & $\$ 0.85$ & 1 \\
\hline & Freq Multiplier & MiniCircuits & MiniCircuits & KSX2-722+ & - & $\$ 6.95$ & 1 \\
\hline & 15dB Gain Block & Analog Devices & Digikey & ADL5541 & 8 LFCSP_VD & $\$ 4.02$ & 1 \\
\hline & TX PA & Avago & Digikey & MGA-545P8 & 8-WFDFN & $\$ 3.70$ & 1 \\
\hline & Power Splitter & MiniCircuits & MiniCircuits & $\overline{\mathrm{GP} 2 \mathrm{X}+}$ & - & $\$ 1.49$ & 1 \\
\hline & Coupler & MiniCircuits & MiniCircuits & SYBD-16-53HP+ & - & $\$ 29.95$ & 2 \\
\hline & \begin{tabular}{|l|} 
BPF \\
\end{tabular} & TDK & Digikey & DEA453960BT-3007B1 & - & $\$ 4.50$ & 3 \\
\hline & LNA & Avago & Digikey & MGA-71543 & SOT-343 & $\$ 2.23$ & 2 \\
\hline & 20dB Gain Block & Analog Devices & Digikey & ADL5542 & 8 LFCSP_VD & $\$ 4.02$ & 2 \\
\hline & Mixer & MiniCircuits & MiniCircuits & SIM-762H+ & - & $\$ 8.95$ & 2 \\
\hline & LPF Op Amp & TI & Digikey & $\overline{\mathrm{LMH} 6639}$ & 8 pin SOIC & $\$ 1.67$ & 2 \\
\hline & VGA & Analog Devices & Digikey & AD603 & 8pin SOIC & $\$ 10.97$ & 2 \\
\hline & Regulator $(3.3 v, 3 v)$ & Micrel & Digikey & MIC5335-SPYMT TR & - & $\$ 2.31$ & 1 \\
\hline \multirow[b]{13}{*}{ Resistors } & $3 \mathrm{k}$ ohm & Panasonic & Digikey & ERJ-6ENF3001V & 805 & $\$ 0.10$ & 1 \\
\hline & $2.2 \mathrm{k} \mathrm{ohm}$ & \begin{tabular}{|l|} 
Panasonic \\
\end{tabular} & Digikey & ERJ-3EKF2201V & 603 & $\$ 0.10$ & 1 \\
\hline & $1.24 \mathrm{k} \mathrm{ohm}$ & Panasonic & Digikey & ERJ-3EKF1241V & 603 & $\$ 0.10$ & 2 \\
\hline & $1 \mathrm{k}$ ohm & Panasonic & Digikey & ERJ-3EKF1001V & 603 & $\$ 0.10$ & 2 \\
\hline & 196 ohm & \begin{tabular}{|l|} 
Panasonic \\
\end{tabular} & Digikey & ERJ-6ENF1960V & 805 & $\$ 0.10$ & 2 \\
\hline & 124 ohm & \begin{tabular}{|l} 
Panasonic \\
\end{tabular} & Digikey & ERJ-3EKF1240V & 603 & $\$ 0.10$ & 2 \\
\hline & $113 \mathrm{ohm}$ & Panasonic & Digikey & ERJ-3EKF1130V & 603 & $\$ 0.10$ & 2 \\
\hline & 51 ohm & Panasonic & Digikey & ERJ-6ENF51ROV & 805 & $\$ 0.10$ & 2 \\
\hline & 49.9 ohm & Panasonic & Digikey & ERJ-6ENF49R9V & 805 & $\$ 0.10$ & 7 \\
\hline & 27 ohm & Panasonic & Digikey & ERJ-6ENF27ROV & 805 & $\$ 0.10$ & 1 \\
\hline & $20 \mathrm{ohm}$ & Panasonic & Digikey & ERJ-3EKF20ROV & 603 & $\$ 0.10$ & 2 \\
\hline & 0 ohm & Panasonic & Digikey & ERJ-6GEYOROOV & 805 & $\$ 0.05$ & 50 \\
\hline & 0 ohm & Panasonic & Digikey & ERJ-3GEYOROOV & 603 & $\$ 0.02$ & 15 \\
\hline \multirow[b]{7}{*}{ Inductors } & $47 \mathrm{nH}$ & \begin{tabular}{|l} 
Murata \\
\end{tabular} & Digikey & LQG18HN47NJ00D & 603 & $\$ 0.24$ & 3 \\
\hline & $4.7 \mathrm{nH}$ & Murata & Digikey & LQG18HN4N7S00D & 603 & $\$ 0.21$ & 3 \\
\hline & $3.9 \mathrm{nH}$ & Murata & Digikey & LQG18HN3N9SO0D & 603 & $\$ 0.23$ & 4 \\
\hline & $2.9 \mathrm{nH}$ & Abracon & Digikey & ATFC-0402-2N9-BT & 402 & $\$ 0.08$ & 1 \\
\hline & $2.7 \mathrm{nH}$ & \begin{tabular}{|l|} 
Murata \\
\end{tabular} & Digikey & LQG18HN2N7SOOD & 603 & $\$ 0.21$ & 2 \\
\hline & $2.2 \mathrm{nH}$ & Murata & Digikey & LQG18HN2N2SO0D & 603 & $\$ 0.21$ & 3 \\
\hline & $1.5 \mathrm{nH}$ & Murata & Digikey & LQG18HN1N5S00D & 603 & $\$ 0.21$ & 4 \\
\hline \multirow[b]{22}{*}{ Capacitors } & $1 \mathrm{uF}$ & Murata & Digikey & GRM188R61A105KA61D & 603 & $\$ 0.10$ & 13 \\
\hline & $0.1 \mathrm{uF}$ & Murata & Digikey & GRM21BR71E104KA01L & 805 & $\$ 0.10$ & 1 \\
\hline & $0.1 \mathrm{uF}$ & Murata & Digikey & GRM188R71H104KA93D & 603 & $\$ 0.10$ & 16 \\
\hline & $0.01 \mathrm{uF}$ & Murata & Digikey & $\overline{\text { GRM188R71H103KA01D }}$ & 603 & $\$ 0.27$ & 8 \\
\hline & $1200 \mathrm{pF}$ & Murata & Digikey & GRM1885C1H122JA01D & 603 & $\$ 0.12$ & 3 \\
\hline & $1000 \mathrm{pF}$ & Murata & Digikey & GRM1885C1H102JA01D & 603 & $\$ 0.10$ & 5 \\
\hline & $390 \mathrm{pF}$ & Murata & Digikey & GRM1885C1H391JA01D & 603 & $\$ 0.10$ & 2 \\
\hline & $100 \mathrm{pF}$ & Murata & Digikey & $\overline{\text { GRM2165C1H101JA01D }}$ & 805 & $\$ 0.12$ & 2 \\
\hline & $100 \mathrm{pF}$ & Murata & Digikey & GRM1885C1H101JA01D & 603 & $\$ 0.10$ & 8 \\
\hline & $68 \mathrm{pF}$ & Murata & Digikey & GRM1885C1H680JA01D & 603 & $\$ 0.10$ & 7 \\
\hline & $47 \mathrm{pF}$ & Murata & Digikey & $\overline{\text { GRM21A5C2E470JW01D }}$ & 805 & $\$ 0.29$ & 2 \\
\hline & $33 \mathrm{pF}$ & Murata & Digikey & GRM21A5C2E330JW01D & 805 & $\$ 0.29$ & 6 \\
\hline & $22 \mathrm{pF}$ & Murata & Digikey & GRM1885C1H220JA01D & 603 & $\$ 0.10$ & 1 \\
\hline & $18 \mathrm{pF}$ & Murata & Digikey & GRM1885C1H180JA01D & 603 & $\$ 0.10$ & 2 \\
\hline & $12 \mathrm{pF}$ & Murata & Digikey & GRM1885C1H120JA01D & 603 & $\$ 0.10$ & 1 \\
\hline & $10 \mathrm{pF}$ & Murata & Digikey & GRM1885C1H100JA01D & 603 & $\$ 0.10$ & 8 \\
\hline & $6.8 \mathrm{pF}$ & Murata & Digikey & GRM1885C1H6R8DZ01D & 603 & $\$ 0.10$ & 3 \\
\hline & $4.3 p F$ & Murata & Digikey & GRM1885C1H4R3CZ01D & 603 & $\$ 0.10$ & 3 \\
\hline & $3.3 \mathrm{pF}$ & Murata & Digikey & GQM2195C2E3R3BB12 & 805 & $\$ 1.37$ & 2 \\
\hline & $2.4 \mathrm{pF}$ & Murata & Digikey & GRM1885C1H2R4CZ01D & 603 & $\$ 0.10$ & 3 \\
\hline & $0.8 \mathrm{pF}$ & Murata & Digikey & GJM1555C1HR80BB01 & 402 & $\$ 0.20$ & 8 \\
\hline & $0.5 \mathrm{pF}$ & Murata & Digikey & GRM1885C1HR50CZ01D & 603 & $\$ 0.10$ & 1 \\
\hline \multirow[b]{2}{*}{ Connectors } & SMA-F Connector & Emerson & Digikey & $142-0701-806$ & - & $\$ 4.84$ & 3 \\
\hline & Test Point Hook & Keystone Elec. & Digikey & 5016 & - & $\$ 0.38$ & 16 \\
\hline
\end{tabular}


APPENDIX C: Transmitter Level Plan

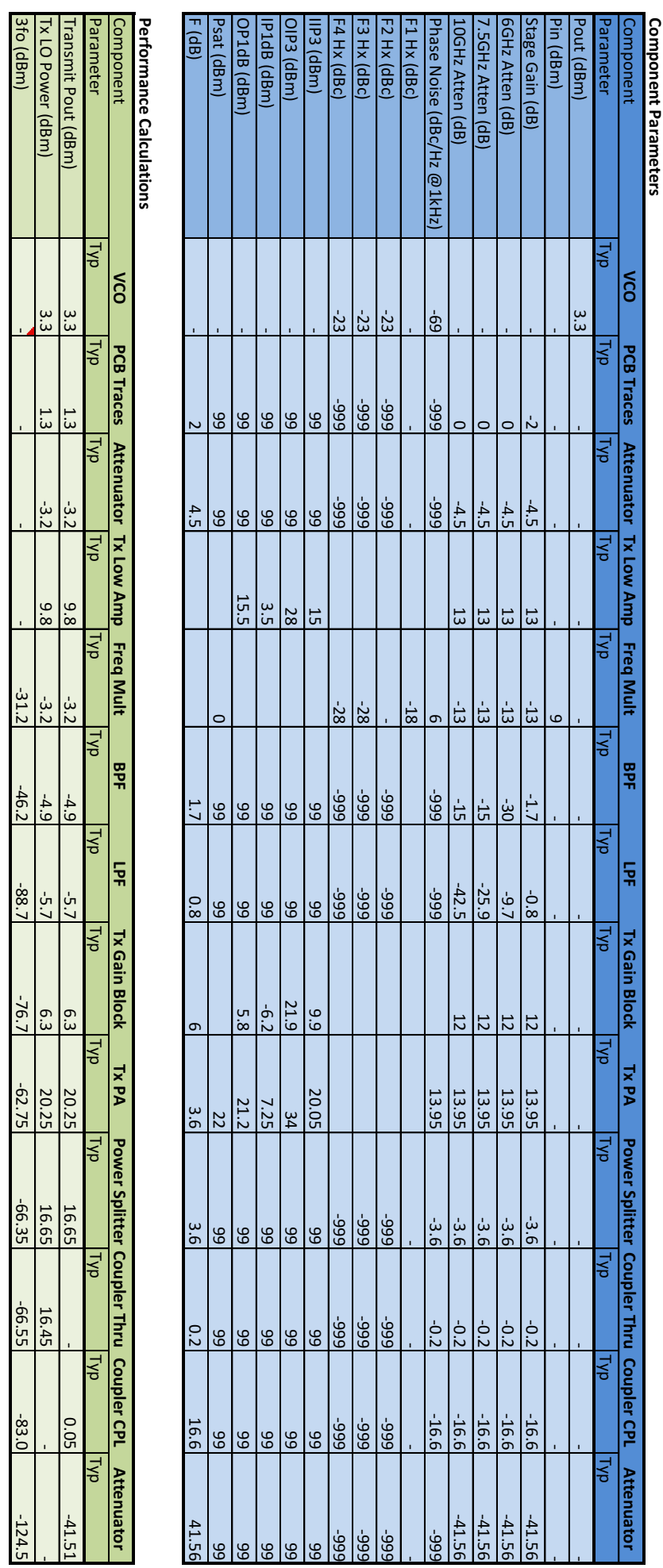


APPENDIX D: Receiver Level Plan

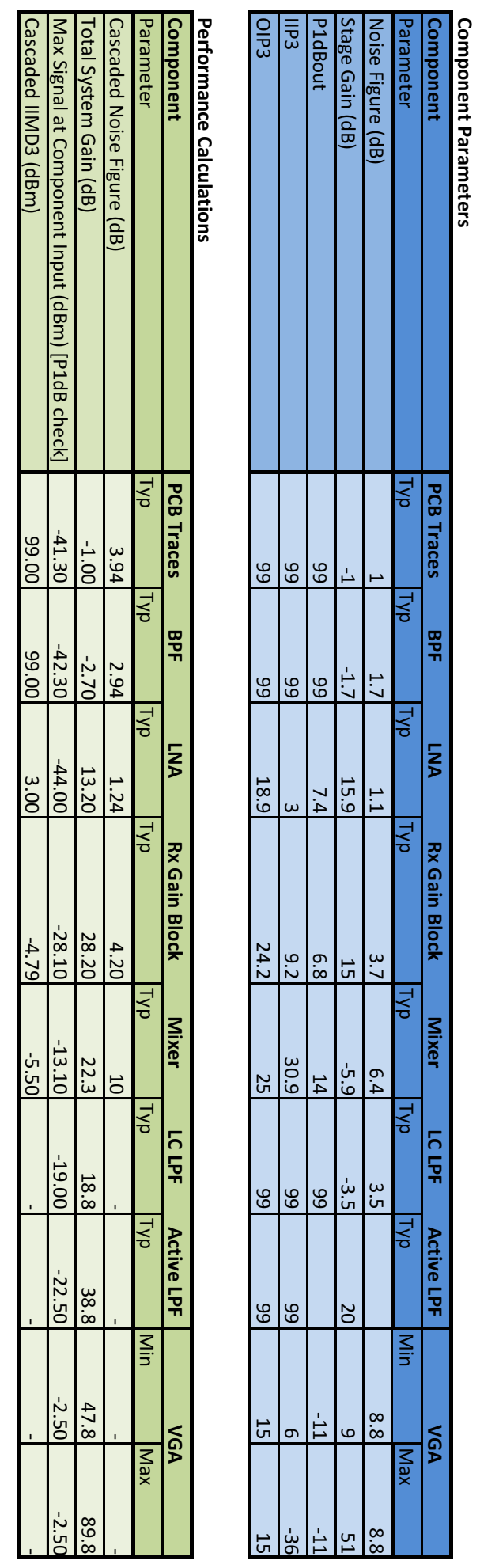




\section{APPENDIX E: 1.3uH Wideband Bias Choke}

Coilcraft $4310 \mathrm{LC}-132 \mathrm{KE}$ is a $1.3 \mathrm{uH}$ wideband bias choke. It's self-resonant frequency is at $235 \mathrm{MHz}$, with a $15.1 \mathrm{~m} \Omega$ max DC resistance. This component was used to remove the resonant suck outs in the gain of the various amplifiers, by providing isolation at RF frequencies between the amplifiers and the DC bias network. Figure E.0.1 below shows the impedance of the RF choke across frequency. When placed in series with the amplifier bias, it provides isolation up to $6 \mathrm{GHz}$.

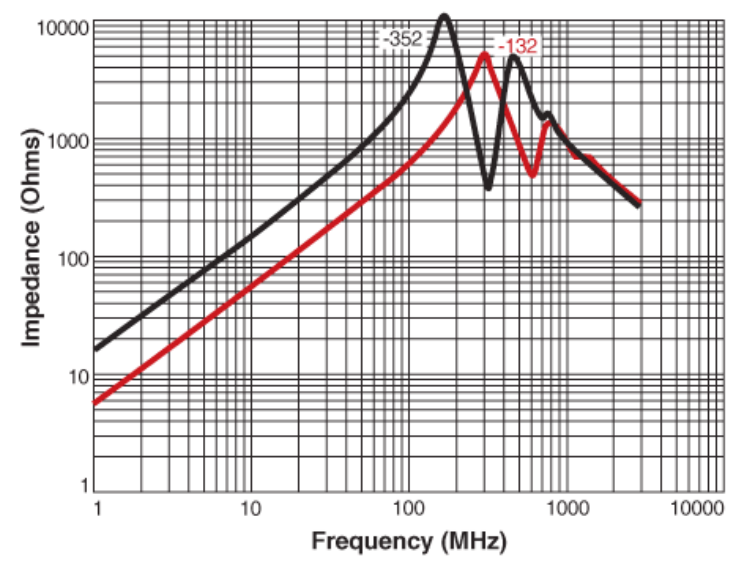

Figure E.0.1: Wideband Choke Impedance 
APPENDIX F: LNA RF Input Matching
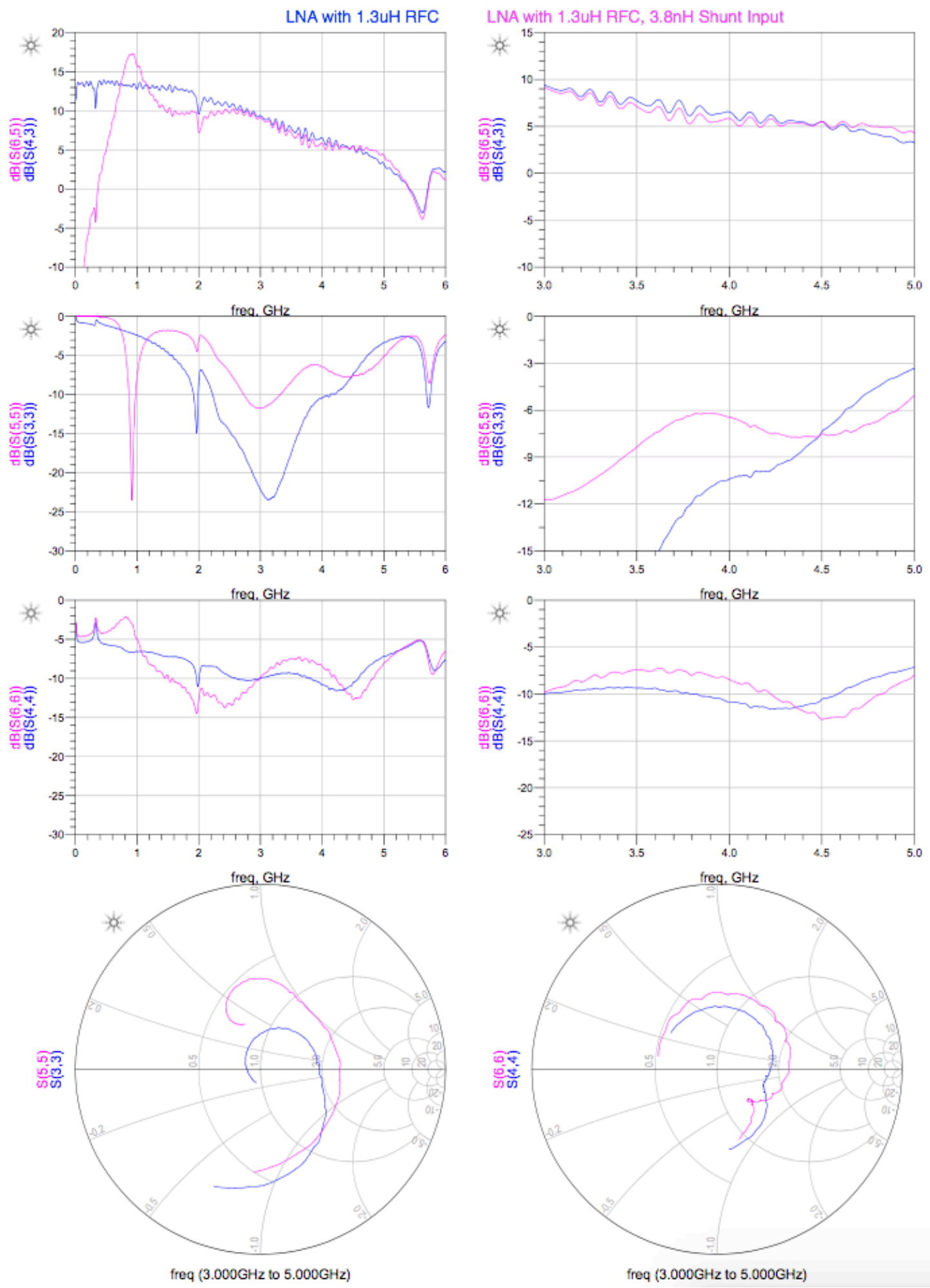


\section{APPENDIX G: Rx Gain Block RF Output Matching}
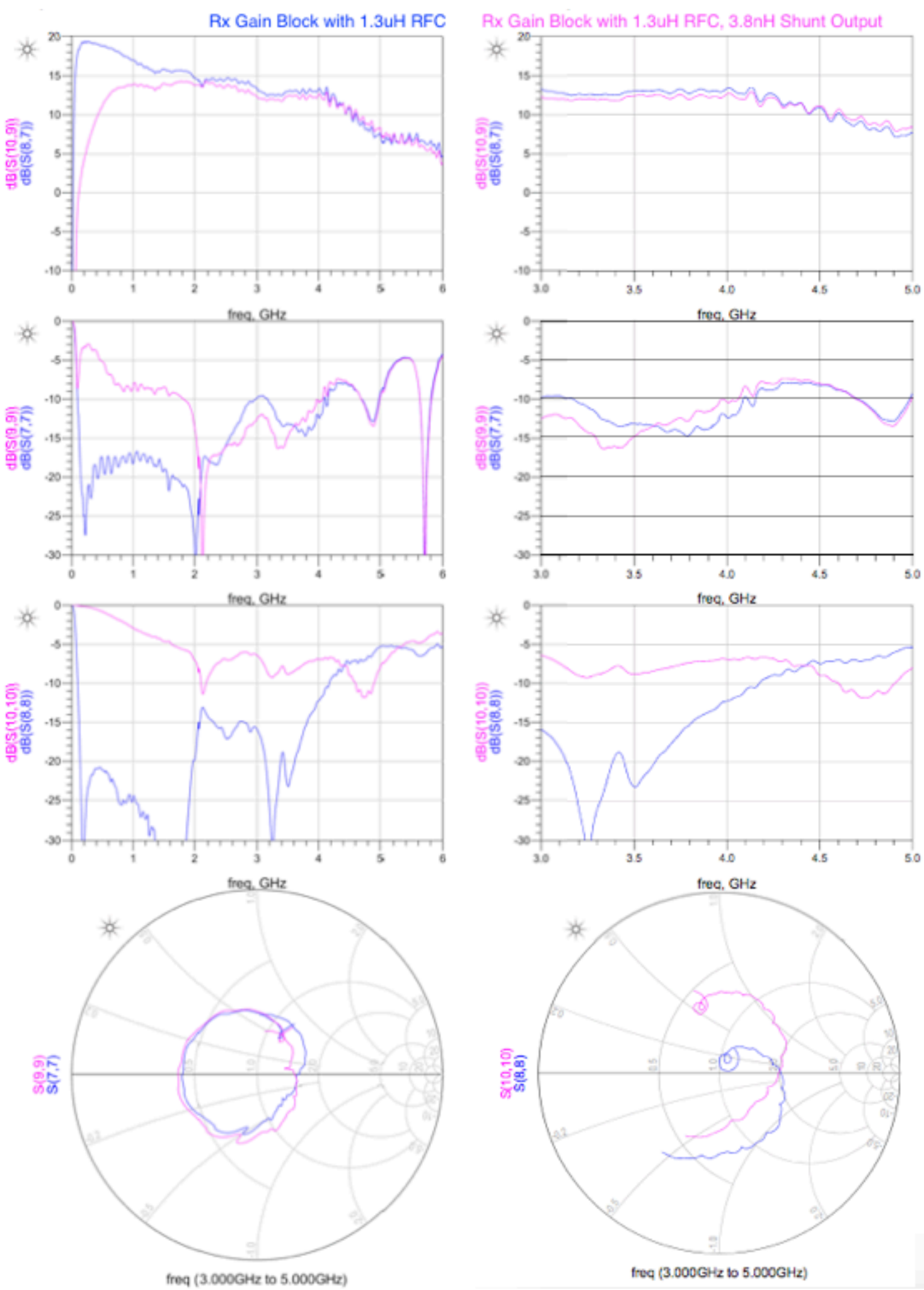
APPENDIX H: Reference FR4 Transmission Line Data

A reference microstrip transmission line printed on FR4 is measured for comparison.

The one foot long through line is pictured below in Figure H.0.1.

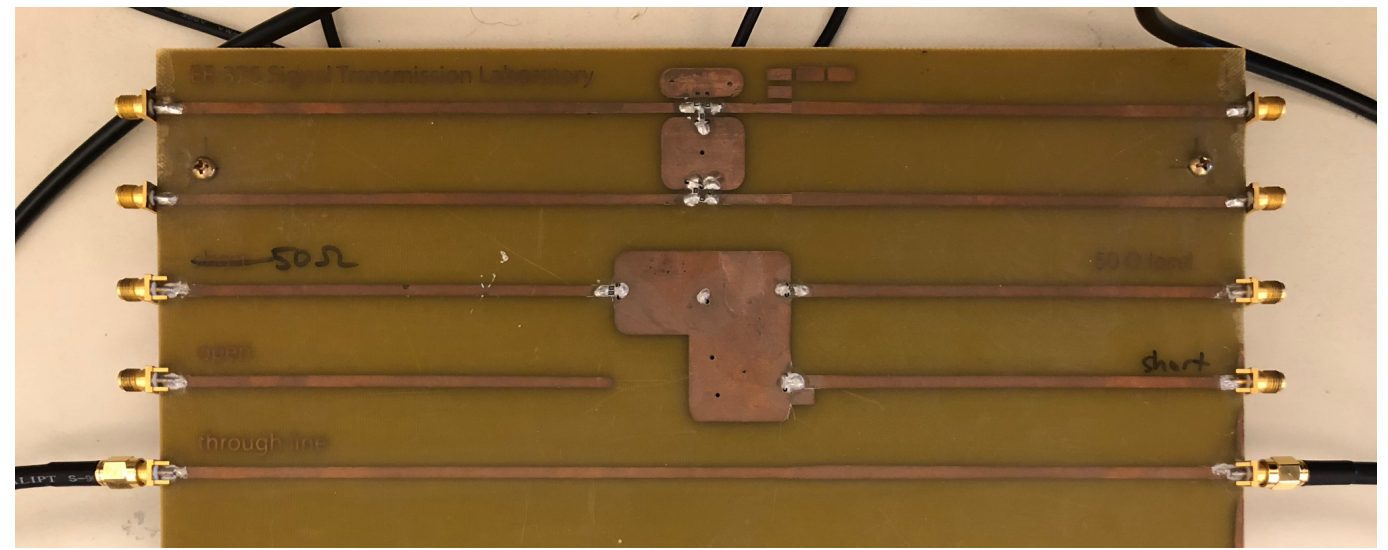

Figure H.0.1: Measured Reference FR4 Microstrip Transmission Line

Figure H.0.2 shows the insertion loss of the one foot trace up to $9 \mathrm{GHz}$. At $3 \mathrm{GHz}$ the line has $2.99 \mathrm{~dB}$ insertion loss, and at $4.9 \mathrm{GHz}$ it has $5.15 \mathrm{~dB}$. The insertion loss increases fairly linearly across frequency as expected.

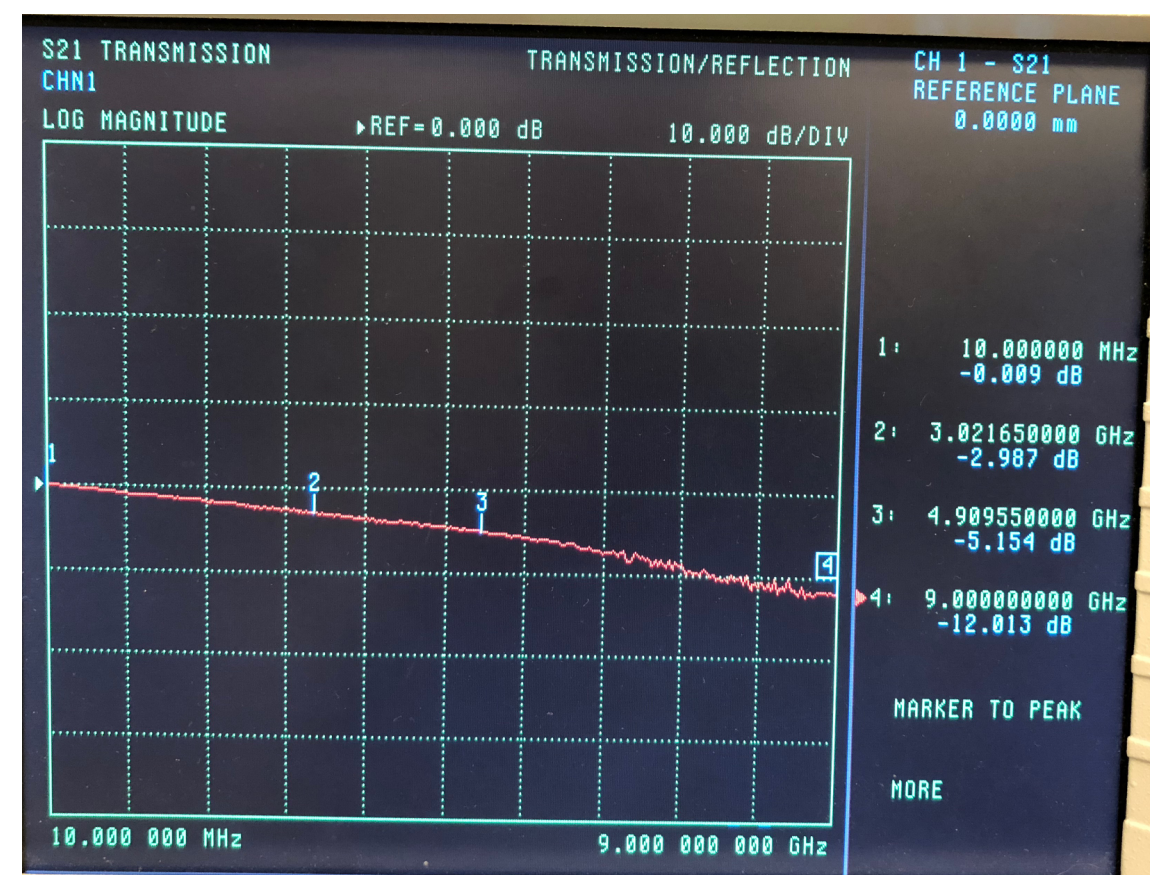

Figure H.0.2: Measured Reference FR4 Transmission Line Insertion Loss 
The trace return loss and impedance is shown below in Figure H.0.3. The return loss from $3-5 \mathrm{GHz}$ remains better than $20 \mathrm{~dB}$, before degrading around $8 \mathrm{GHz}$.

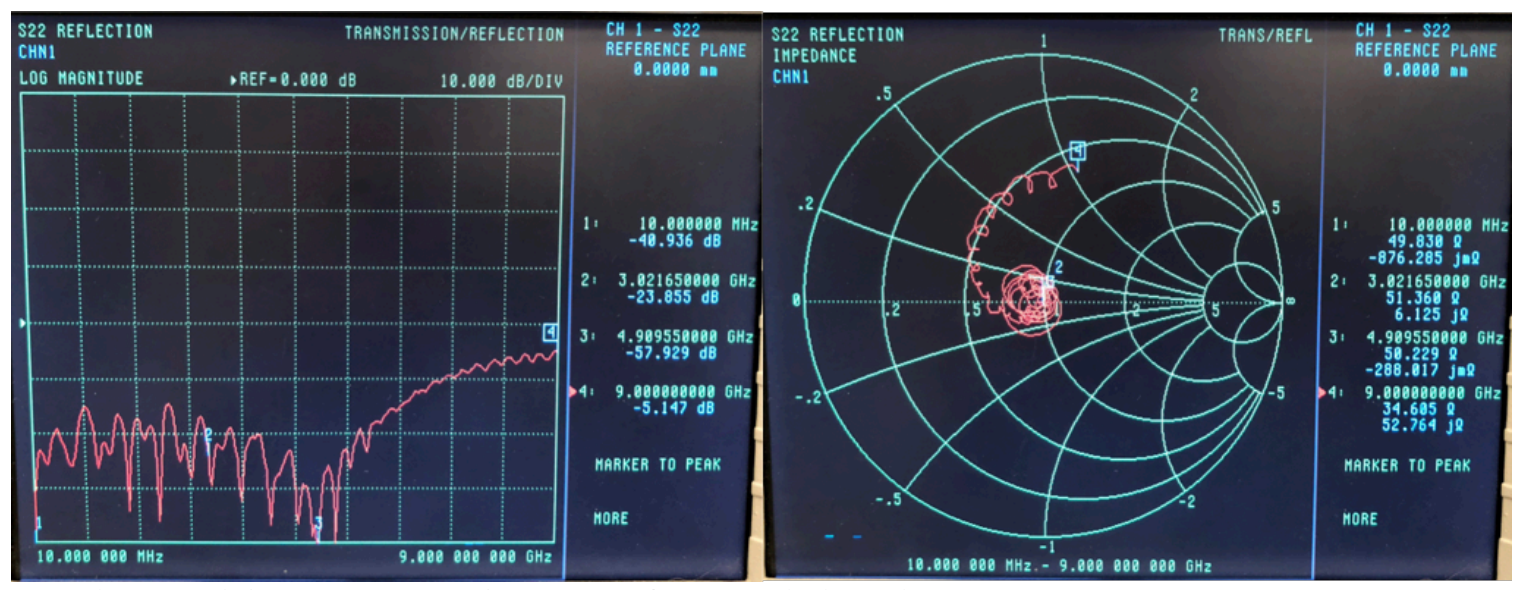

Figure H.0.3: Measured Reference FR4 Transmission Line RL and Impedance

The reference transmission line demonstrates that FR 4 can be used to create $50 \Omega$ microstrip traces at high frequency. The high frequency edge of the UWB system operates at 4.9GHz, where the data shows a $50 \Omega$ characteristic impedance and $5.15 \mathrm{~dB}$ of insertion loss over the one foot length. 


\section{APPENDIX I: Environmental Impact and Sustainability}

The proposed monitoring system is meant to improve on existing methods for eldercare monitoring, and further facilitate extending independent living. Extending independent living conserves resources by relaxing pressure on the healthcare system and allowing individuals to live within their own means for longer. One of the improvements for proposed system compared to current methods the reduction of required hardware for a monitoring system. A FMCW UWB radar system allows continuous monitoring of multiple rooms in all conditions with a single unit. The silhouette capture RF front-end designed here consists of off the shelf components, a fabricated PCB, and soldering assembly. The components chosen are RoHS compliant, meaning they do not contain lead, mercury, or other hazardous materials as defined by the Restriction of Hazardous Substances Directive 20002/95/EC. 